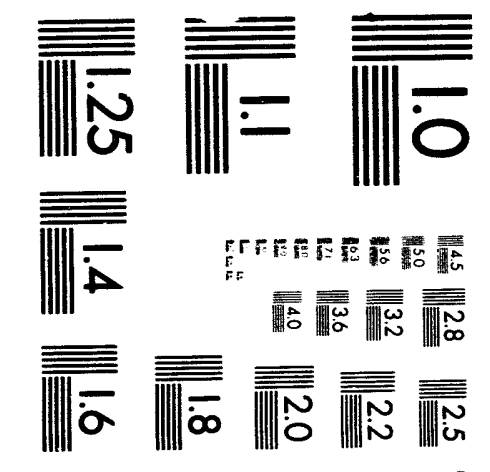



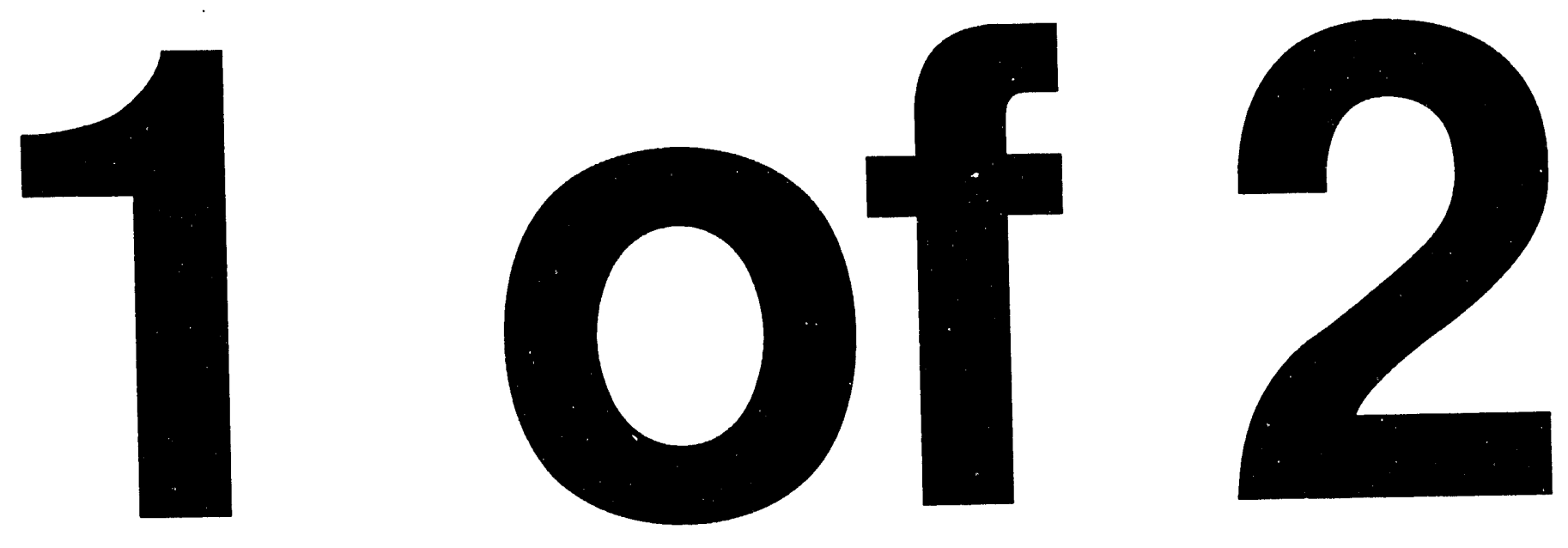
$.94 y<x$

UCRL-ID-115027

\section{CRITICALITY SAFETY EVALUATION FOR PORTSMOUTH X-345 HIGH-ENRICHED-URANIUM STORAGE AREA}

Brian L. Koponen

September 20, 1993

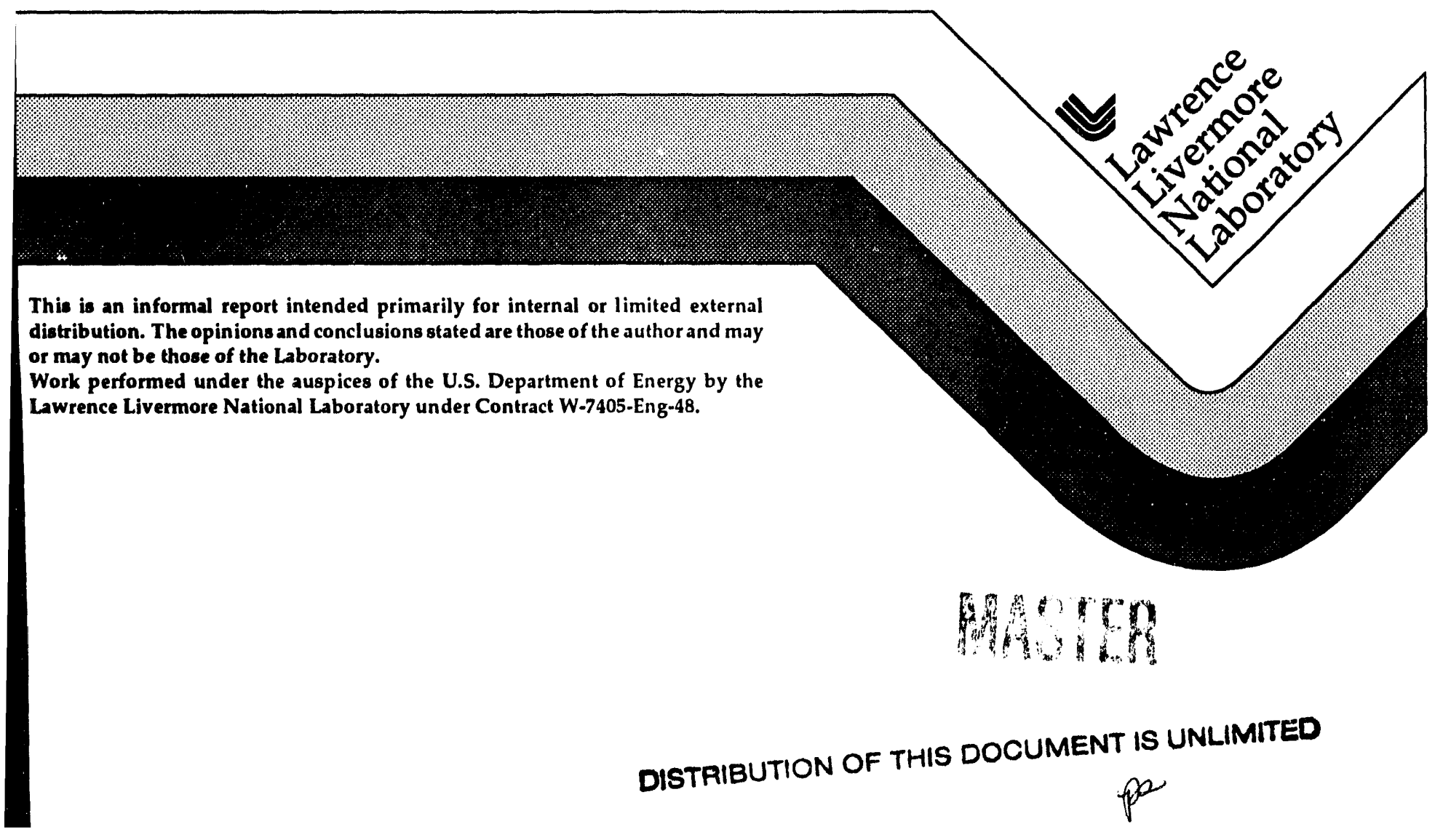




\section{DISCLAIMER}

This document was prepared as an account of work sponsored by an agency of the United States Government. Neither the United States Government nor the University of Califonia nor any of their employees, makes any warranty, express or implied, or assumes any legal liability or responsibility for the accuracy, completeness, or usefulness of any information, apparatus, product, or process disclosed, or represents that its use would not infringe privately owned rights. Reference herein to any specilic commercial products, process, or service by trade name, trademark, manufacturer, or otherwise, does not necessarily constitute or imply its endorsement, recommendation, or favoring by the United States Government or the University of California. The views and opinions of authors expressed herein do not necessarily st ate or reflect those of the United States Government or the University of California, and shall not be used for advertising or product endorsement purposes.

This report has been reproduced directly from the best available cony.

Available to DOE and DOE contractors from the Omine of Scientitic and Teclunical Information P.O. Box 62, Oak Ridge, 'TN 37831

Prices availahle from (615) $576-8401$, FTs 626-8401

Available to the public from the

National Technical Information Service

U.S. Departericut of Commerce 5285 l'ort Royal Rd., Springlicld, VA 22161 


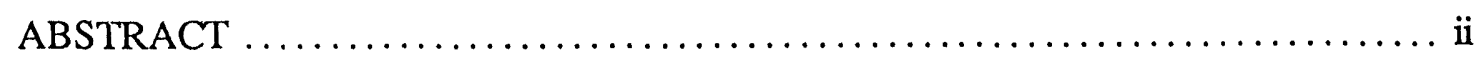

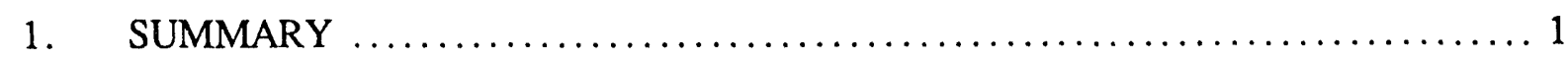

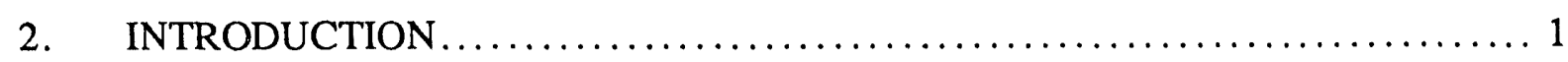

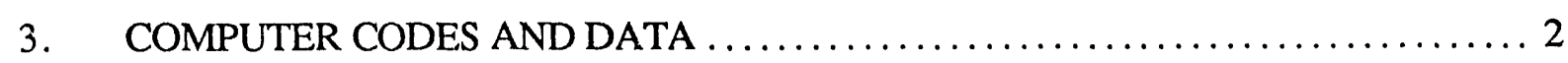

4. MATERIAL FORMS, CONTAINERS, AND STORAGE $\ldots \ldots \ldots \ldots \ldots \ldots \ldots \ldots$

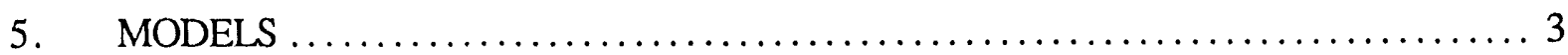

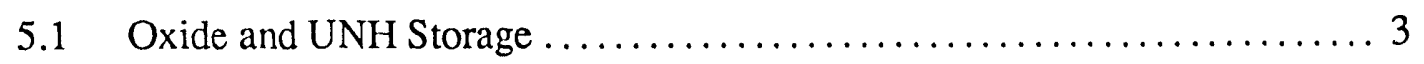

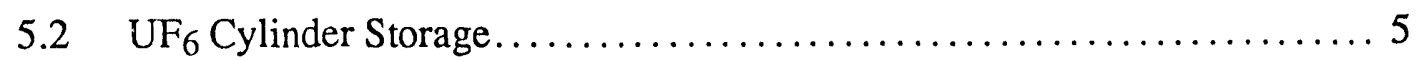

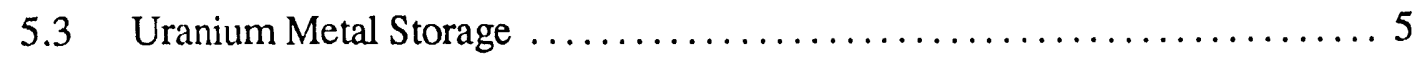

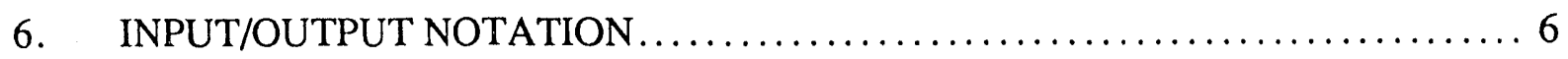

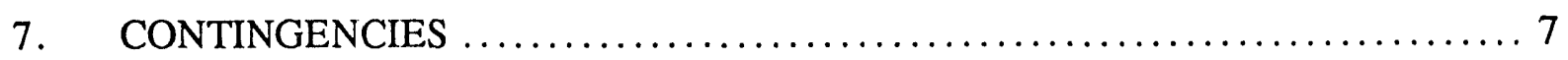

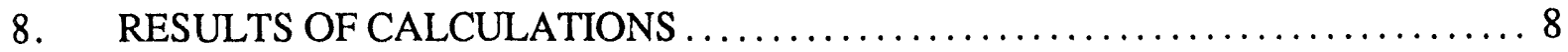

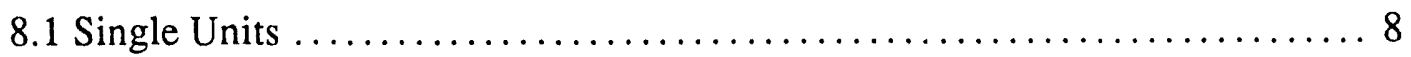

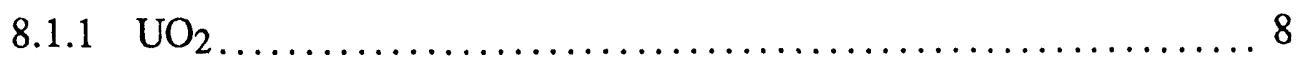

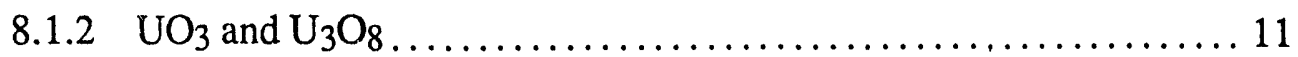

8.1 .3 Container Breaching ................................ 11

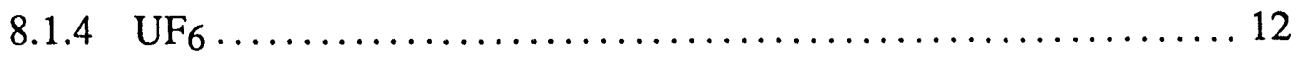

8.2 Arrays.............................................. 14

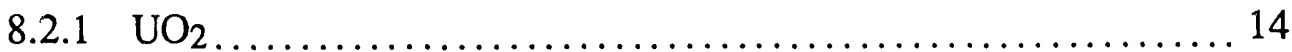

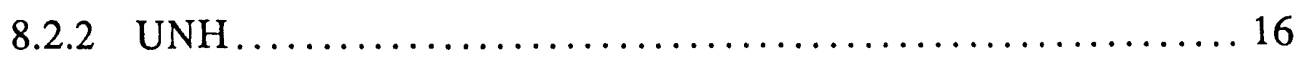

8.2.3 Hydrogen Content in Concrete ......................... 16

9. CONCLUSIONS AND RECOMMENDATIONS $\ldots \ldots \ldots \ldots \ldots \ldots \ldots \ldots \ldots \ldots$

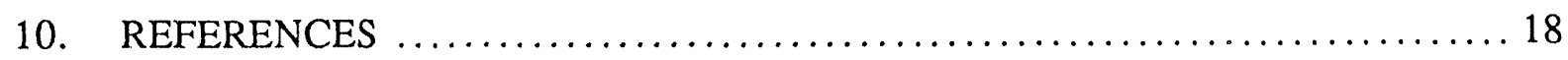

APPENDIX A Documentation on X-345 Criticality Calculations................ A-1

APPENDIX B KENO V.a/SCALE Job Control Description .................... B-1

APPENDIX C Confirmatory Calculations with MCNP $\ldots \ldots \ldots \ldots \ldots \ldots \ldots \ldots . . \ldots \ldots \ldots$

APPENDIX D KENO Computer Input Spreadsheets ....................... D-1

APPENDIX E KENO Results Summary ............................. E-1

APPENDIX F $\quad$ KENO Problem Inputs ................................. F-1

APPENDIX G Moderation Controls for Uranium Oxides ..................... G-1 


\begin{abstract}
This report evaluates nuclear criticality safety for the High-Enriched Uranium storage area of the X-345 building of the Portsmouth Gaseous Diffusion Plant. The effects of loss of moderation or mass control are examined for storage units in or out of the storage receptacles. Recommendations are made for decreasing criticality hazards under some conditions of storage or handling considered to be hazardous.
\end{abstract}


September 20, 1993

\section{UES Technical Report: \\ Results of Criticality Safety Evaluation for Portsmouth X-345 High Enriched Uranium Storage Area}

\section{Summary}

This report summarizes the results of a criticality safety evaluation of the Portsmouth Gaseous Diffusion Plant High-Enriched Uranium (HEU) storage. There have been occasions in the past when water entered some of the storage receptacles. Since moderation control is important in the safe handling of high-enriched materials this has led to the need for a reexamination of criticality safety in the facility. We were asked to cletermine whether the double contingency principle applies under water flooding or other conditions encountered by containers in storage or handling. This study shows that loss of moderation control or exceeding uranium oxide mass limits could possibly result in criticality. We see, however, that double-contingency requirements can be met if the high-enriched uranium oxides are stored in 5-inch, rather than 6-inch-diameter canisters.

Results of this study confirm that criticality safety depends upon maintaining the $\mathrm{UF}_{6}$ in a dry condition. We recommend use of cylinders with diameters equal to or less than that recommended by ANSI/ANS-8.1-1983.1

Criticality is possible, regardless of container diameter, if a container is open or breached when water enters a receptacle, mixes with the fissile material, and the mixture disperses into the larger volume of the receptacle. This hazard can be minimized by filling the void space surrounding the containers with non-porous filler material.

Adoption of the above recommendations would help assure compliance with DOE orders which require that whenever practicable, reliance be placed on equipment design in which dimensions are limited rather than on administrative controls.

\section{Introduction}

This Technical Report includes the results of studies done for DOE Task 33-91-15, Job ID X-1, in conformance to Part 1 of Activity Plan for X-345 Sub task, outlined in GDPNCS92-05 2 which states:

LLNL will establish a firm computational basis for normal and off-normal operations in $X-345$ using a configuration controlled version of KENO V.a. Calculations will be made for single units and infinite planar arrays. Confirmatory calculations may be made with $M C N P$ if appropriate. $L L N L$ will identify the double contingency controls and investigate the effects of double batching. To support the PRA effort assessing water intrusion, some $K E N O$ calculations will be made as a function of depth of water in the storage receptacle. Appendix A lists Portsmouth letters and reports which support efforts to set the design limits and administrative controls for the X-345 facility. 


\section{Computer Codes and Data}

The Activity Plan referenced above states that a configuration-controlled version of KENO V.a will be used. We used the computer system maintained by the Computing and Telecommunications Division of Martin Marietta Energy Systems, Inc. at Oak Ridge, Tennessee. Connections to Oak Ridge were made via Internet from ncsc, permitting efficient transfers of input and output files. The ORNL computer has an Internet address of 128.219.128.149 (a.k.a. NX10A.CTD.ORNL.GOV). For this study we used KENO V.a CSAS4 in SCALE4.1, with the 27-group cross section library, running on the IBM 3090 system. Validation calculations ${ }^{3}$ supporting this version of SCALE/KENO, which were done by MMES, show that the criterion for subcriticality should be that $k_{\text {eff }}+2 \sigma+$ bias $\leq 0.95$. The KENO default PARAM values were used. A sample Job Control description is included in Appendix B.

We also did confirmatory calculations with the Los Alamos Monte Carlo code, MCNP, using the X6XS.0 cross section set derived from the ENDF/B-V cross section library. MCNP was installed on the LLNL Fission Energy \& Systems Safety Program computer, ncsc, by LANL staff members who validated the installation with their set of test problems. The MCNP calculations provide additional assurance of the validity of our KENO modeling. Appendix C summarizes the MCNP calculations.

Documentation on the software used for this study is listed in Table 1.

Table 1. Computer Software Documentation.

\begin{tabular}{|l|l|l|l|}
\hline \multicolumn{1}{|c|}{ Author } & \multicolumn{1}{|c|}{ Report } & Date & \multicolumn{1}{c|}{ Title } \\
\hline \hline Parks, C. B., Ed. & $\begin{array}{l}\text { NUREG/CR-0200, Rev. } \\
\text { 4, ORNL/NUREG/CSD- } \\
\text { 2/R4, Vols. I, II, and III }\end{array}$ & $\begin{array}{l}\text { April } \\
1992\end{array}$ & $\begin{array}{l}\text { SCALE: A Modular Code System for } \\
\text { Performing Standardized Computer } \\
\text { Analyses for Licensing Evaluation }\end{array}$ \\
\hline $\begin{array}{l}\text { Petrie, L. M. and } \\
\text { Landers, N. F. }\end{array}$ & $\begin{array}{l}\text { NUREG/CR-0200, Rev. } \\
\text { 4, ORNL/NUREG/CSD- } \\
\text { 2/R4, Vol. 2, Sec. F11 }\end{array}$ & $\begin{array}{l}\text { April } \\
1992\end{array}$ & $\begin{array}{l}\text { KENO V.a: An Improved Monte Carlo } \\
\text { Criticality Program with Supergrouping }\end{array}$ \\
\hline $\begin{array}{l}\text { Bucholz, J. A., et } \\
\text { al. }\end{array}$ & NUREG/CR.-0200, Rev. & $\begin{array}{l}\text { April } \\
\text { 4, ORNL/NUREG/CDs- } \\
\text { 2/R4, Vol. 3, Sec. M8 }\end{array}$ & Standard Composition Library \\
\hline $\begin{array}{l}\text { Briesmeister, } \\
\text { Judith F. }\end{array}$ & LA-7396-M, Rev. 2 & Sept. & MCNP-A General Monte Carlo Code \\
& & 1986 & for Neutron and Photon Transport \\
\hline $\begin{array}{l}\text { Pruvost, Norman } \\
\text { L., et al. }\end{array}$ & LA-12135-MS & June & The X6XS.0 Cross Section Library for \\
\hline Koponen, B. L. & LLNL Internal & Feb. & Installation of MCNP on NCIS Sun \\
& Memorandum NCIS/041 & 1992 & \\
\hline
\end{tabular}




\section{Material Forms, Containers, and Storage}

High-enriched ${ }^{a}$ materials that are permitted to be stored in the HEU area include uranium solutions, ${ }^{\text {b }}$ uranium metal, uranyl nitrate hexahydrate (UNH), $\mathrm{UF}_{6}$, and uranium oxides: $\mathrm{UO}_{2}$, $\mathrm{UO}_{3}$ and $\mathrm{U}_{3} \mathrm{O}_{8}$. $\mathrm{UF}_{6}$ is stored in monel cylinders. Polybottles are used for $\mathrm{UNH}$ and uranium solution storage. The containers for the other materials are steel canisters $5 \mathrm{in.,} 6 \mathrm{in}$., $8 \mathrm{in}$., and 12 in. in diameter. The canisters are described in GAT/GDP-1073.4 The X-345 underground storage facility is described in PGDP drawings X-345-S17, X-345-S18, and X-345-A10.

5. Models

\subsection{Oxide and UNH Storage}

In KENO V.a we modeled the canisters, receptacles, and surrounding concrete using the drawings referenced in Section 4 and other sources as specified. Appendix D includes the Excel spreadsheets used to determine various material densities used in the KENO V.a models. The polybottles and steel-walled canisters are set into steel-lined receptacles which are surrounded by concrete on the sides and bottom. To simulate water flooding we placed water in the void region surrounding the canister. In some problems, we also placed water inside the canister in order to simulate admission of water through damaged or corroded canister walls, or other inadvertent violation of moderation controls. Figure 1 shows a schematic representation of the uranium oxide receptacle region with a canister inserted. For single unit calculations we placed this unit into a 100 -cm-edge concrete cube with the lid centered in and flush with a cube face. (100-cm is thick enough to be effectively infinite.) Table 2 includes the dimensions of the components of the model shown in Fig. 1.

Uranium oxides are currently being stored in 6-inch diameter containers, however-when our calculations showed double contingency requirements are not being met for mass and moderation control-we also examined 5-inch containers for uranium oxides.

For array calculations we set up a model which closely represents the actual storage area. In this model we used the KENO V.a "hole geometry" option to model the triangular pitch of the receptacles in the below-ground storage area. A unit cell was modeled such that the use of $\mathrm{X}$ and $\mathrm{Y}$ reflection boundary conditions in KENO results in a simulation of an infinite planar array. The model is sketched in Fig. 2. Constituents of the "X-345" concrete were determined by an analysis which is summarized in GAT-DM-1444, Rev. $1.5^{5}$ We did some comparison calculations with different concrete models and found differences in $\mathrm{k}_{\mathrm{eff}}$ of less than $2 \%$, as was found also in earlier Portsmouth calculations reported in POEF-T-34896. Materials stored in the containers are described in Appendix D, Tables D1-D8. We obtained densities for $\mathrm{UO}_{2}$ and $\mathrm{UO}_{3}$ from the Atlantic-Richfield Criticality Handbook, ${ }^{7}$ page III.B.2-2. Densities for $\mathrm{U}_{3} \mathrm{O}_{8}$ are determined using ORNL/CSD/TM-284, Appendix A, Eq. A.1. Densities for UNH are from the Atlantic-Richfield Criticality Handbook ${ }^{7}$ and from the Handbook of Inorganic Chemistry. ${ }^{8}$

a The enrichment assumed in this study was $97.65 \%$ 235U as reported in POEF-T-3447 and in other documents. The detailed weight percents are as follows: ${ }^{234} \mathrm{U}: 1.50 \%$; ${ }^{235} \mathrm{U}: 97.65 \%$; ${ }^{238} \mathrm{U}: 0.85 \%$.

b Concern for criticality in the event of leakage of a polybottle into a storage receptacle led facility management to move solutions containing more than $350 \mathrm{gm}{ }^{235} \mathrm{U}$ to another building. 


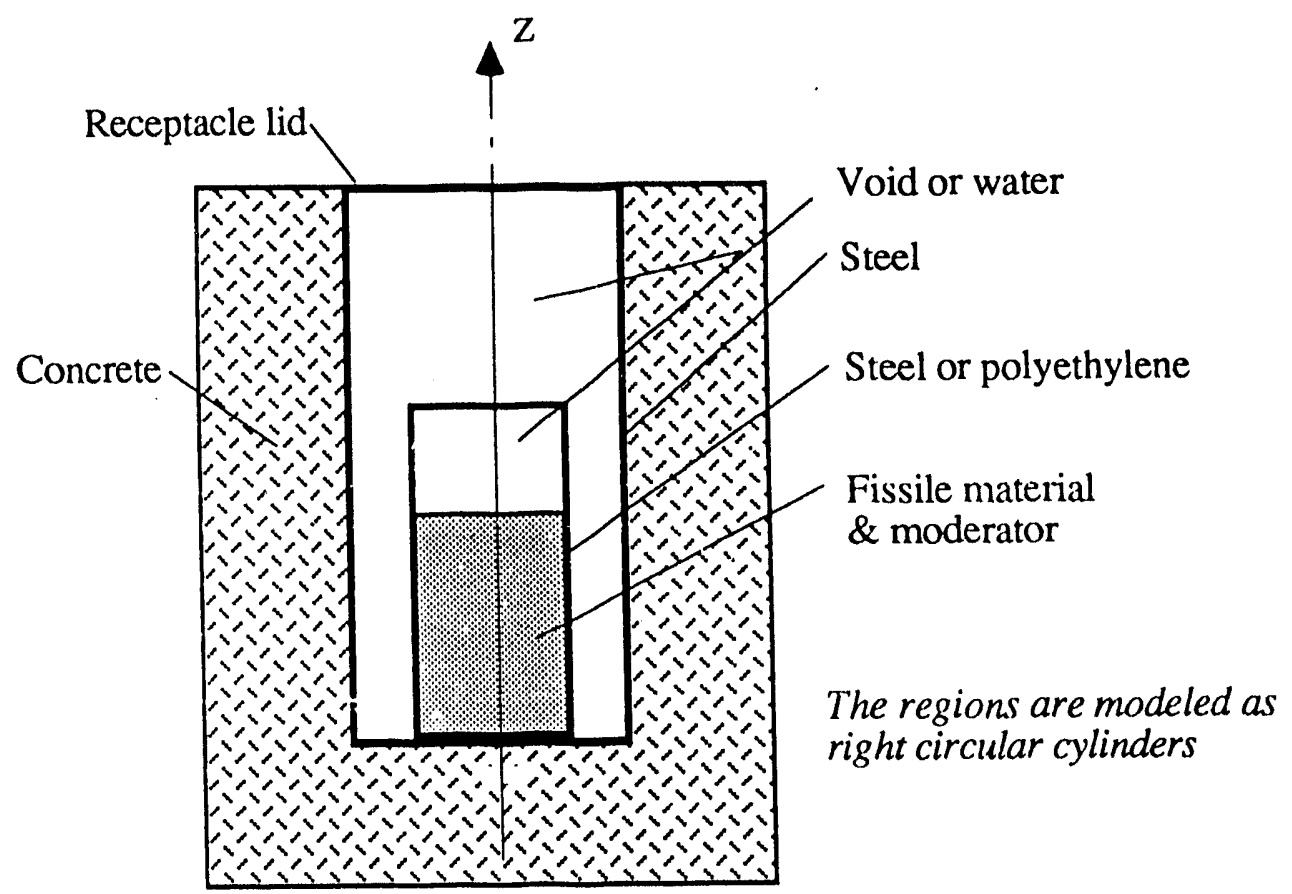

Fig. 1. Schematic of X-345 oxide and UNH receptacle region modeled in KENO V.a. (not to scale)

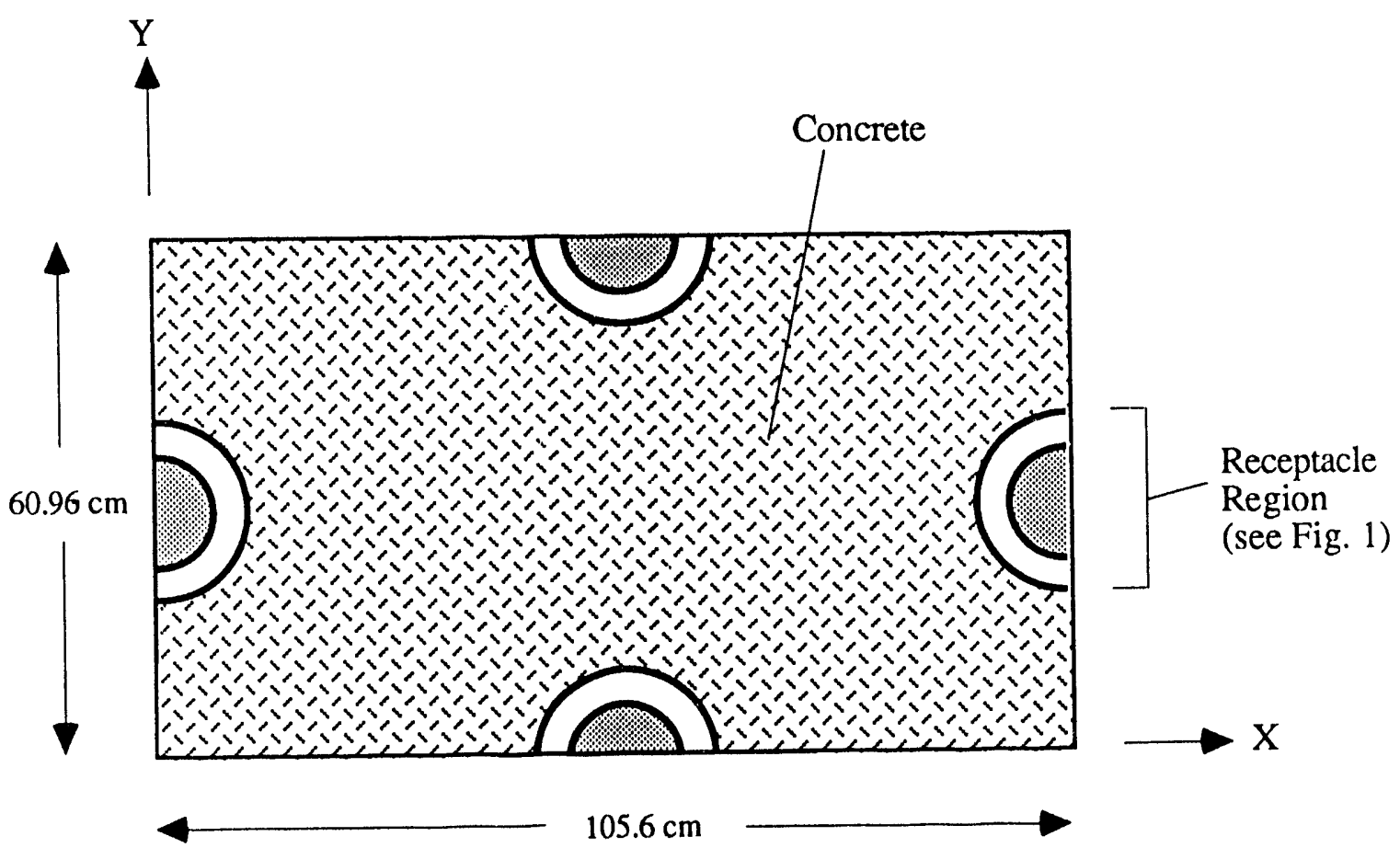

Fig. 2. Schematic of unit cell used in KENO V.a array calculations (not to scale). Reflection $\mathrm{X} \& \mathrm{Y}$ boundary conditions are used to simulate an infinite planar array. 
Table 2. X-345 uranium oxide and UNH storage receptacle materials. ${ }^{\text {a }}$

\begin{tabular}{|l|l|l|l|}
\hline Region & $\begin{array}{l}\text { Materials in } \\
\text { Region }\end{array}$ & $\begin{array}{l}\text { Radius } \\
(\mathbf{c m})\end{array}$ & $\begin{array}{l}\text { Length } \\
(\mathbf{c m})\end{array}$ \\
\hline \hline Canister & carbon steel ${ }^{\mathrm{b}}$ & $\begin{array}{l}7.699 \text { or } \\
6.429 \mathrm{e}\end{array}$ & $38.258^{\mathrm{e}}$ \\
\hline Canister contents & $\begin{array}{l}\mathrm{UO}_{2}, \mathrm{UO}_{3}, \mathrm{U}_{3} \mathrm{O}_{8}, \\
\text { void, water }\end{array}$ & $\begin{array}{l}7.62 \text { or } \\
6.35^{\mathrm{e}}\end{array}$ & $38.1^{\mathrm{e}}$ \\
\hline Polybottlec & Polyethylene & $5.97375^{\mathrm{e}}$ & $45.829 \mathrm{e}$ \\
\hline Polybottle contents & UNH & $5.8738^{\mathrm{e}}$ & $45.72^{\mathrm{e}}$ \\
\hline Receptacle interior & void, water & $10.95375^{\mathrm{f}}$ & $67.31^{\mathrm{f}}$ \\
\hline Receptacle & carbon steel & $11.24585^{\mathrm{f}}$ & $69.215^{\mathrm{f}}$ \\
\hline
\end{tabular}

a The matcrials are described in detail in Appendix D, Tables D1-D8.

${ }^{b}$ Steel canisters are used for uranium oxide storage.

c Polybottles are used for UNH storage.

$\mathrm{d}$ The base is $0.635 \mathrm{~cm}$ thick and the lid is $1.27 \mathrm{~cm}$ thick.

e Dimensions obtained from Rich Boelens, department engineer for uranium materials handling, PGDP.

f Dimensions from drawing X-345-S18, Rev. 1.

\section{2 $\mathrm{UF}_{6}$ Cylinder Storage}

We also examined the storage of 8-inch $\mathrm{UF}_{6}$ cylinders. Our KENO model is described in Fig. 3 and in Table 3. ${ }^{\mathrm{a}}$ The initial loading of the cylinder is 255 pounds of solid UF6. Various amounts of water are added at the top of the cylinder, producing $\mathrm{UO}_{2} \mathrm{~F}_{2}$ hydrate $($ at $\mathrm{H} / \mathrm{U}<4$ ), or $\mathrm{UO}_{2} \mathrm{~F}_{2}$ solution (at $\mathrm{H} / \mathrm{U} \geq 4$ ). We used the density relationships reported by Jordan and Turner in ORNL/CSD/TM-284, Appendix A. ${ }^{9}$ (Note that the density of $235 \mathrm{U}$ in the $\mathrm{UO}_{2} \mathrm{~F}_{2}$ hydrates $(\mathrm{H} / \mathrm{U}<4)$ is greater than that for solid $U 6$.) Appendix $D$, Table D9 shows the material compositions used in the KENO V.a problems.

\subsection{Uranium Metal Storage}

Current limits ${ }^{4}$ for uranium metal storage in X-345 include the following:

1. A container or several containers with multiple pieces of uranium metal may be stored in a receptacle if the total $235 \mathrm{U}$ mass is less than $3 \mathrm{~kg}$.

2. A container with a single piece of uranium metal may be stored in a receptacle if the $235 \mathrm{U}$ mass is less than $10 \mathrm{~kg}$.

3. The above mass limits do not apply if the uranium metal containers have an inside diameter of 2.7 inches or less; however, the container must have physical supports to center it in the receptacle.

4. As with other non-UF6 compounds, if more than $8 \mathrm{~kg} 235 \mathrm{U}$ is in a receptacle, the surrounding storage spaces must be empty.

These limits assure that the mass of uranium metal is well below that permitted by American National Standard ANSI/ANS-8.1-1983. ${ }^{1}$ We thus did not consider it necessary to do additional studies for uranium metal in the storage receptacles.

a Our KENO runs inadvertently used a monel thickness equal to the oxide canister wall thickness rather than the 3/16-inch value shown in the drawing. The KENO problems were later rerun with a PC version of KENO. 


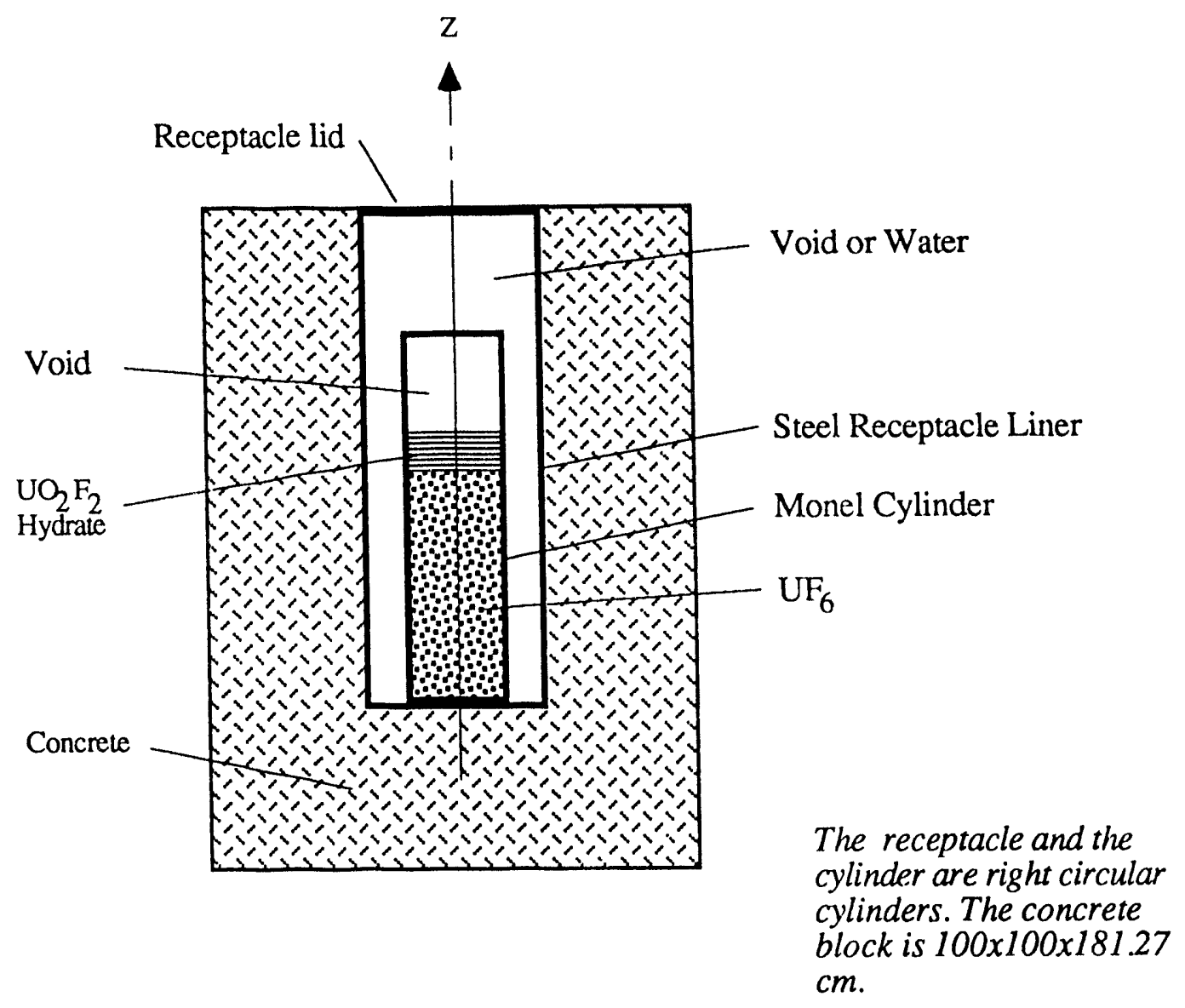

Fig. 3. Schematic of X-345 $\mathrm{UF}_{6}$ receptacle region modeled in KENO V.a (not to scale)

Table 3. X-345 materials for 8 in.-diameter $\mathrm{UF}_{6}$ cylinder calculations.

\begin{tabular}{|c|c|c|c|}
\hline Region & Materials in Region & Radius (cm) & Length (cm) \\
\hline Cylinder & monel & $10.6273^{a}$ & $125.41^{\mathrm{a}}$ \\
\hline Cylinder Contents ${ }^{b}$ & $\mathrm{UF}_{6}, \mathrm{UO}_{2} \mathrm{~F}_{2}, \mathrm{H}_{2} \mathrm{O}$ & $10.16^{a}$ & $124.4756^{a}$ \\
\hline Receptacle ${ }^{c}$ & carbon steel & $17.78^{\mathrm{c}}$ & $151.765^{c}$ \\
\hline Receptacle Interior & void, water & $17.2244^{d}$ & $149.86^{d}$ \\
\hline
\end{tabular}

a Dimensions from Portsmouth drawing CX-761-M2012

$\mathrm{b}$ The materials are described in detail in Appendix D, Table D-9.

c The receptacle base is $0.635 \mathrm{~cm}$ thick and the lid is $1.27 \mathrm{~cm}$ thick.

$\mathrm{d}$ Dimensions from Portsmouth drawing X-345-S18, Rev. 1.

\section{Input/Output Notation}

Each of the KENO runs prepared for this study is identified by a Problem ID which incorporates a coding which provides an overview of the problem characteristics. The explanation of the coding is included in Appendix E. Appendix E also includes a summary of the KENO results. The KENO problem inputs are included in Appendix F. 


\section{Contingencies}

Appendix A of American National Standard ANSI/ANS-8.1-1983 ${ }^{1}$ includes examples of variations in process controls that should be considered in criticality safety studies. Discussions with Portsmouth's staff lead us to believe that contingencies which must be considered in the vault areas should include the items listed in Table 4.

Table 4. X-345 vault contingencies.

\begin{tabular}{|l|l|}
\hline \multicolumn{1}{|c|}{ ANSI/ANS-8.1-1983 } & \multicolumn{1}{c|}{ X-345 Vault Application } \\
\hline \hline $\begin{array}{l}\text { A change in intended shape or dimensions } \\
\text { resulting from bulging, corrosion, or bursting } \\
\text { of a container, or failure to meet specifications } \\
\text { in fabrication. }\end{array}$ & $\begin{array}{l}\text { Canisters may be dropped, punctured, suffer } \\
\text { from corrosion, damaged in fires, etc. }\end{array}$ \\
\hline $\begin{array}{l}\text { An increase in the mass of fissionable material } \\
\text { in a location as the result of operational error, } \\
\text { improper labeling, equipment failure, or failure } \\
\text { of analytical techniques. }\end{array}$ & $\begin{array}{l}\text { Administrative or analytical error could result } \\
\text { in mass limits being exceeded. A canister could } \\
\text { receive more material than allowed, up to } \\
\text { complete filling. }\end{array}$ \\
\hline $\begin{array}{l}\text { A change in the ratio of moderator to } \\
\text { fissionable material resulting from: } \\
\text { (a) Inaccuracies in instruments or chemical } \\
\text { analyses, }\end{array}$ & $\begin{array}{l}\text { Moderators may be mixed with the fissile } \\
\text { material in excess of the allowances. Materials } \\
\text { exposed to moist air could absorb water. Water } \\
\text { (b) Flooding, spraying, or otherwise supplying } \\
\text { units or groups of units with water, oil, snow } \\
\text { (i.e., low-density water), cardboard, wood, or } \\
\text { other moderating material. }\end{array}$ \\
$\begin{array}{l}\text { surface sources and mix with the fissile } \\
\text { material if canisters are improperly sealed or } \\
\text { breached due to breakage, puncture, or } \\
\text { corrosion. (The history of water intrusion } \\
\text { events for the X-345 vault is included in }\end{array}$ \\
\hline
\end{tabular}

In our computations we model the canisters in their normal state, but also simulate some violations of controls-specifically, mass, moderation, and reflection. In the present study we do not determine the probabilities of these abnormal conditions occurring. (A separate probabilistic analysis, outlined in GDPNCS- $92-05,2$ will be documented separately). The four stages of water moderation that we modeled are included in Table 5.

Table 5. Various stages of water moderation conditions modeled for HEU vault.

\begin{tabular}{|c|l|}
\hline Stage & \multicolumn{1}{c|}{ Description } \\
\hline 1 & $\begin{array}{l}\text { Water enters a receptacle, but the canister is intact and there is no } \\
\text { contact between the fissile material and water. } \\
\text { Water enters a receptacle and subsequently into the canister, but the } \\
\text { water remains in the void space in the canister and does not mix with } \\
\text { the fissile material. } \\
\text { Water enters a receptacle and a canister, and mixes with the fissile } \\
\text { material, but the mixture remains confined to the canister volume. } \\
\text { Water enters a receptacle and into a canister and the fissile material is } \\
\text { dispersed into the volume of the receptacle. }\end{array}$ \\
\hline
\end{tabular}


8. Results of Calculations

\subsection{Single Units}

\subsubsection{UO $\mathrm{U}_{2}$}

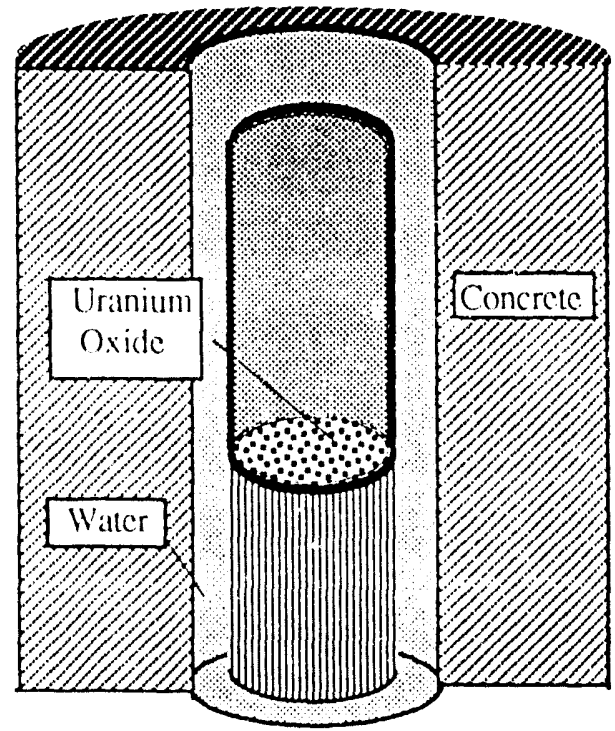

Fig. 4. Schematic of oxide storage receptacle and canister interior.

Although the focus of this study is a storage vault, the subcriticality of any single unit must always be assured-so we began this study with a look at single units, stored as depicted in Fig. 4. In Figs. 5 and 6 we show the results of KENO calculations for single canisters. Fig. 5 shows what can happen when the uranium oxide mass is not controlled. The material densities for the various cases are given in Appendix D, Table D1. In this series of calculations the $\mathrm{H} / \mathrm{U}$ is kept at 10 and the mass of $\mathrm{UO}_{2}$ varies. The void space iti the receptacle is filled with water. The results show that criticality is possible for 6-inch diameter canisters, but not for 5 -inch diameter canisters.

Figure 6, which represents stage 3 of Table 5, shows what can happen if moderation is not controlled. The series begins with a density of 10.96 for crystalline $\mathrm{UO}_{2}$ at $H / U=0$. We assume that water first enters and fills the space in the receptacle outside the canister. We assume that some water gets past the steel and plastic barriers ${ }^{b}$ and then enters the canister and begins to mix with the $\mathrm{UO}_{2}$. In addition, we assume that the water which enters the canister, measured by the water height above initial $\mathrm{UO}_{2}$ level, totally mixes with the $\mathrm{UO}_{2}$, and that the final volume is the sum of the water and $\mathrm{UO}_{2}$ volumes, i.e., no voume changes due to chemical mixing. The $\mathrm{UO}_{2}$ density decreases as the $\mathrm{UO}_{2}$-water volume increases, until the canister is full. ${ }^{c}$ The material densities for the various cases are given in Appendix D, Table D5 (6-inch) and D6 (5-inch). Again, the KENO results show that 6 -inch diameter containers are not safe with water addition, but 5 -inch containers are safe. Although there may be other means of assuring that double contingency requirements are met-we recommend a change from 6-inch diameter containers for HEU storage to 5 -inch diameter containers. This change would help to insure compliance with current standards and DOF: orders" which require that "wherever practicable, reliance be placed on equipment design in which dimensions are limited rather than on administrative controls."

\footnotetext{
"In section 8.1.3 of this report we look at what happens if a container is breached and the fissile material and water are dispersed into the larger volume of the receptacle.

b The canisters are sealed in two plastic bags to reduce the probability of water intrusion.

c In Figs. $5 \& 6$ the dashed line at 0.95 represents the maximum value of $k_{e f f}+2 \sigma+b i a s$ for this version of SCALE/KENO supported by MMES validatation calculations ${ }^{3}$
} 


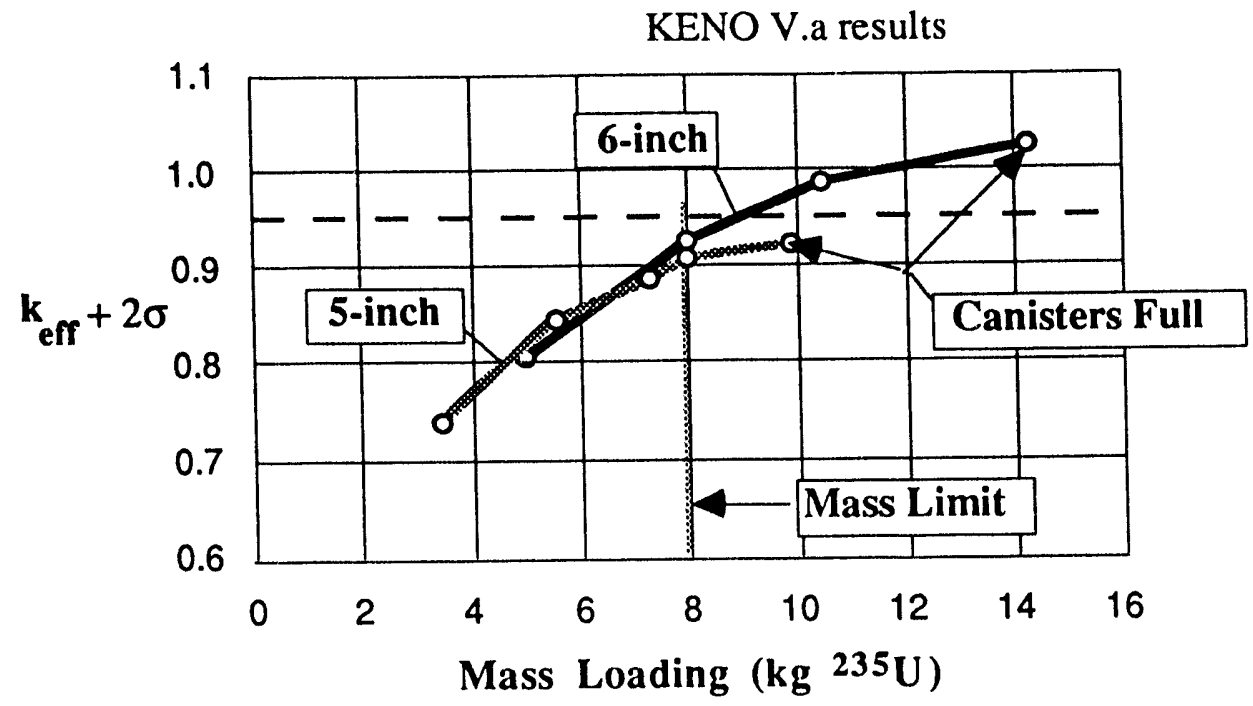

Fig. 5. Variation of $\mathrm{UO}_{2}$ loading for single canisters inside water-flooded storage receptacles. The $\mathrm{H} / \mathrm{U}$ is 10 . The canister loading limit of $8 \mathrm{~kg}$ is indicated. The interior of each canister is unflooded. Results are shown for canisters with diameters of 5 and 6 inches. This is stage 1 of Table 5 . The dashed line gives $k_{\mathrm{eff}}=0.95$.

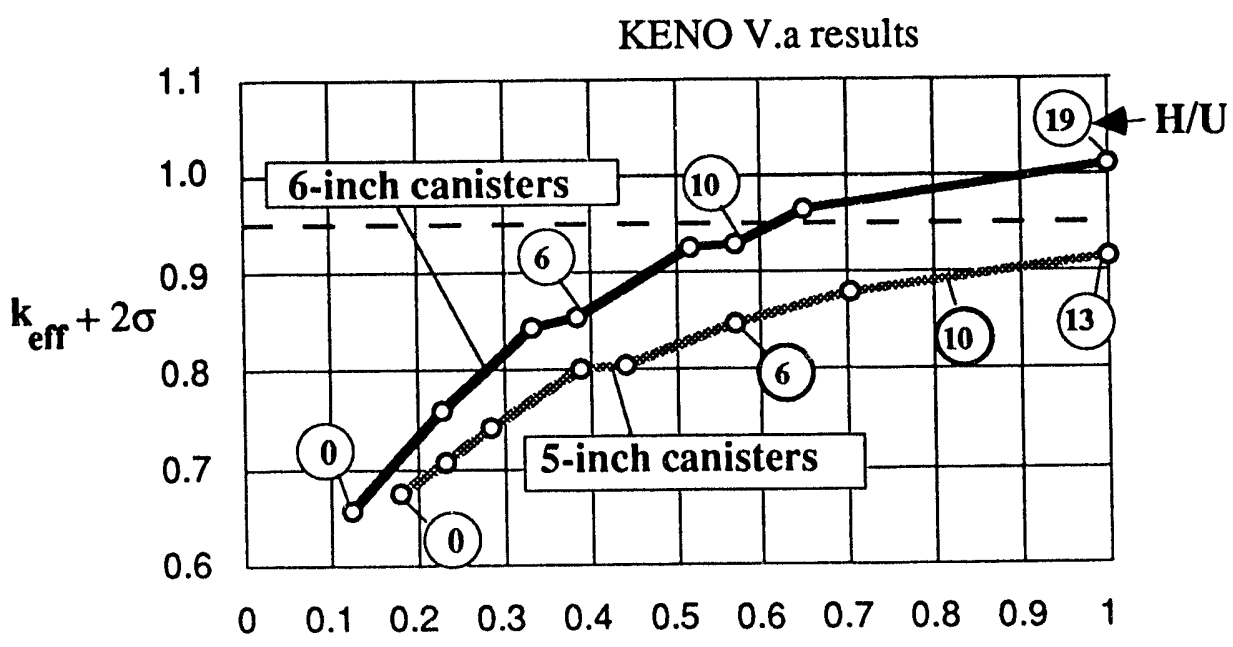

Fraction of canister filled

Fig. 6. Loss of moderation control for single $\mathrm{UO}_{2}$-loaded canisters in storage receptacles for two canister diameters. The mass loading is fixed at $8 \mathrm{~kg} 235 \mathrm{U}$. As water is added to the unmoderated, crystal-density $\mathrm{UO}_{2}$, the $\mathrm{UO}_{2}$ density decreases and the $\mathrm{H} / \mathrm{U}$ and mixture volume increase until the canister is full. The circled numbers show approximate location of several values of $\mathrm{H} / \mathrm{U}$ for the mixed canister contents. This is stage 3 of Table 5 . The dashed line gives $\mathrm{k}_{\text {eff }}=0.95$. 
We also looked at moderation variations, stage 3 in Table 5, for ${ }^{235} \mathrm{U}$ masses less than 8 $\mathrm{kg}$ in single 6-inch diameter canisters. A canister containing a smaller amount of fissile material has room for additional moderator. Figure 7 shows the results of moderation variation for 2,5 , and $8 \mathrm{~kg} 235 \mathrm{U}$ masses. The $8 \mathrm{~kg}$ case exceeds $\mathrm{k}_{\mathrm{eff}}+2 \sigma=0.95 \mathrm{for} \mathrm{H} / \mathrm{U} \cong$ 12 ; the $5 \mathrm{~kg}$ case exceeds $\mathrm{k}_{\mathrm{eff}}+2 \sigma=0.95$ for $\mathrm{H} / \mathrm{U} \cong 20$; and the $2 \mathrm{~kg}$ case exceeds $\mathrm{k}_{\text {eff }}+2 \sigma=$ 0.95 for $\mathrm{H} / \mathrm{U} \cong 60$. The material densities for the various cases are given in Appendix $\mathrm{D}$, Table D5.

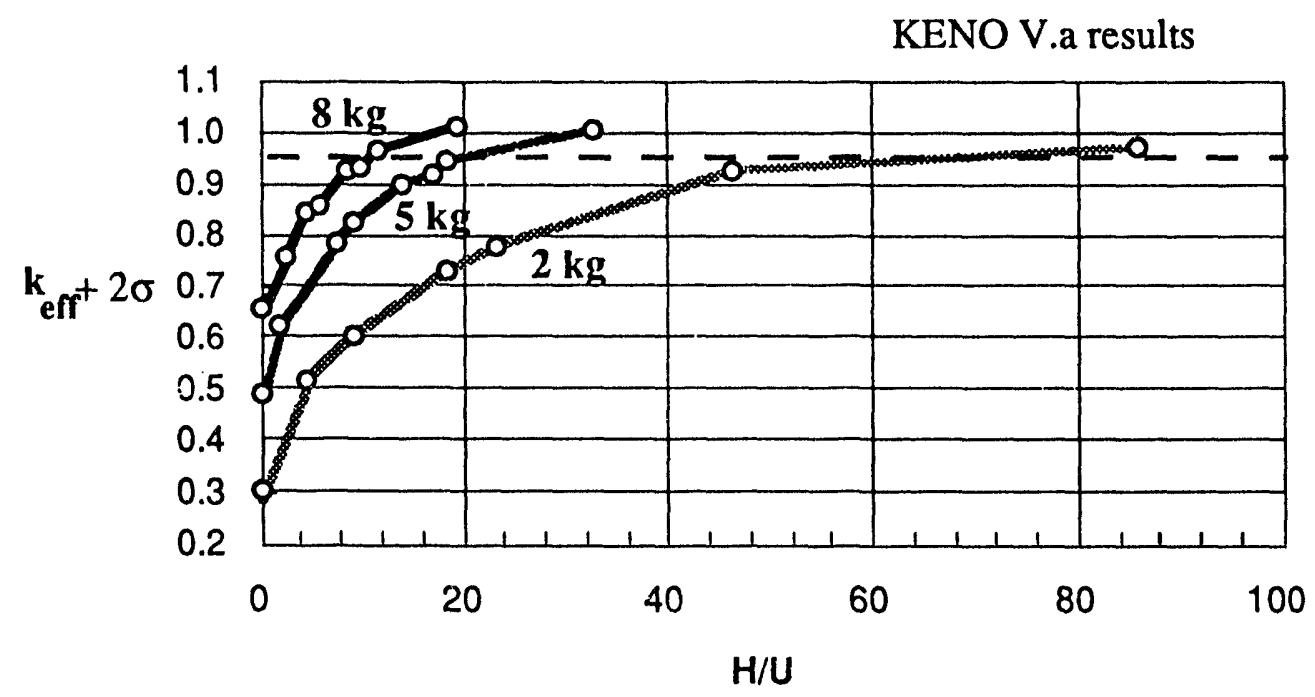

Fig. 7. Loss of moderation control for single 6-inch diameter $\mathrm{UO}_{2}$-loaded canisters in storage receptacles. Three mass loadings are selected. As water is added to the unmoderated, crystal density, $\mathrm{UO}_{2}$ the uranium density decreases and the $\mathrm{H} / \mathrm{U}$ and mixture volume increase until the canister is full. The dashed line gives $\mathrm{k}_{\mathrm{eff}}=0.95$.

Criticality safety is required for units outside of the underground storage area also. Since the canisters are not in the underground storage area $100 \%$ of the time, we did some calculations for units outside of the storage area. If a fissile unit is not closely surrounded in concrete (or other effective reflector) standard practice is to assume that thick water reflection will represent surrounding materials (cf. ANS-8.1-1983, Sec. 5) ${ }^{1}$. Table 6 shows a comparison of KENO V.a results for units inside and outside of storage receptacles. The cases of Table 6 correspond to $\mathrm{UO}_{2}$ units containing ${ }^{235} \mathrm{U}$ and water at the maximum vault allowances as summarized in Appendix G. The first row of Table 6 shows KENO V.a results for canisters immersed in thick water. The second row shows results for the same units inside a storage receptacle which has been flooded by water. The differences in the results are not significant so we assume, therefore, that the flooded receptacle results also apply to units outside of the storage receptacles. The material densities ar given in Appendix D, Table D1. 
Table 6. Results of KENO V.a Calculations for 6-Inch Canisters Containing Maximum Permitted Loadings ${ }^{\mathrm{a}}$ of $\mathrm{UO}_{2}$

\begin{tabular}{|l|c|c|c|}
\hline & \multicolumn{3}{|c|}{$k_{\text {eff }}+\mathbf{2 \sigma}$} \\
\hline \hline$\left(\mathbf{H} / \mathbf{U} ;{ }^{235} \mathbf{U} \text { mass }\right)^{\mathbf{a}}:$ & $\mathbf{6 5 ; 2}$ & $\mathbf{1 5 ; 5}$ & $\mathbf{1 0 ; 8}$ \\
\hline \hline $\begin{array}{l}\text { Canister outside of } \\
\text { receptacle \& immersed } \\
\text { in thick water reflector }\end{array}$ & 0.935 & 0.882 & 0.897 \\
\hline $\begin{array}{l}\text { Canister inside water- } \\
\text { flooded receptacle }\end{array}$ & 0.947 & 0.884 & 0.915 \\
\hline
\end{tabular}

a The canisters contain $\mathrm{UO}_{2}$ at the mass limits described in Appendix G. The materials are described in Table 1 and Appendix D, Table D1.

\subsection{2 $\mathrm{UO}_{3}$ and $\mathrm{U}_{3} \mathrm{O}_{8}$}

It was not necessary to do extensive calculations for all forms of uranium oxides. For a given $\mathrm{H} / \mathrm{U}$ and mass of enriched uranium, $\mathrm{UO}_{2}, \mathrm{UO}_{3}$, and $\mathrm{U}_{3} \mathrm{O}_{8}$ are neutronically about the same (oxygen is merely a diluent). Table 7 shows statistically insignificant differences in the KENO results for the various oxides under comparable conditions, representing stage 3 in Table 5. The material densities are given in Appendix D, Tables D1-D3.

Table 7. Results of KENO V.a calculations for uranium oxide single units in 6-inch canisters.

\begin{tabular}{|c|c|c|}
\hline Materiala $^{\mathbf{a}}$ & $\begin{array}{c}\text { Receptacle } \\
\text { Flooding }\end{array}$ & $\mathbf{k}_{\text {eff }}+\mathbf{2} \sigma$ \\
\hline \hline $\mathrm{UO}_{2}$ & Yes & 0.884 \\
$\mathrm{U}_{3} \mathrm{O}_{8}$ & Yes & 0.878 \\
$\mathrm{UO}_{3}$ & Yes & 0.890 \\
$\mathrm{UO}_{2}$ & No & 0.804 \\
$\mathrm{UO}_{3}$ & No & 0.814 \\
\hline
\end{tabular}

a The $\mathrm{H} / \mathrm{U}$ is 15 and the $235 \mathrm{U}$ mass is $5 \mathrm{~kg}$.

\subsubsection{Container Breaching}

If there should be a water flooding incident involving a receptacle containing highenriched uranium a criticality incident could occur if the fissile material container is breached and fissile material is dispersed into the water outside of the container. The KENO V.a model for this scenario, stage 4 in Table 5, involves admitting water into the container and permitting increasingly larger amounts of $\mathrm{UO}_{2}$ or $\mathrm{UNH}$ to be dispersed throughout the container and receptacle volume, until all of the fissile material is dispersed. The canister for $\mathrm{UO}_{2}$ is 6 inches in diameter and initially holds $8 \mathrm{~kg}{ }^{235} \mathrm{U}$ with $\mathrm{H} / \mathrm{U}=10$. The $\mathrm{UO}_{2}$ material densities are

\footnotetext{
a The maximum loadings are given in MMES Internal Memorandum 504-87-04, in the form of purity (moderator) limits (summarized in Appendix $G$ of this report).

a Similar results can be expected for 5-inch diameter canisters since the 5- and 6-inch canisters $\mathrm{k}$ effectives are close for no dispersal, and for complete dispersal the canister diameter is unimportant.
} 
given in Appendix $\mathrm{D}$, Table $\mathrm{D} 7$. The $\mathrm{UNH}$ container is assumed to contain $\mathrm{UO}_{2}\left(\mathrm{NO}_{3}\right)_{2} \cdot 6\left(\mathrm{H}_{2} \mathrm{O}\right)$ with $5.56 \mathrm{~kg}$ 235U. The UNH material densities are given in Appendix $\mathrm{D}$, Table D8. Figure 8 shows results of calculations. The $\mathrm{UO}_{2}$ case exceeds $\mathrm{k}_{\mathrm{eff}}+2 \sigma=0.95$ for no uranium dispersed. With less than $12 \%$ of the UNH dispersed $k_{\text {eff }}+2 \sigma$ exceeds 0.95 .

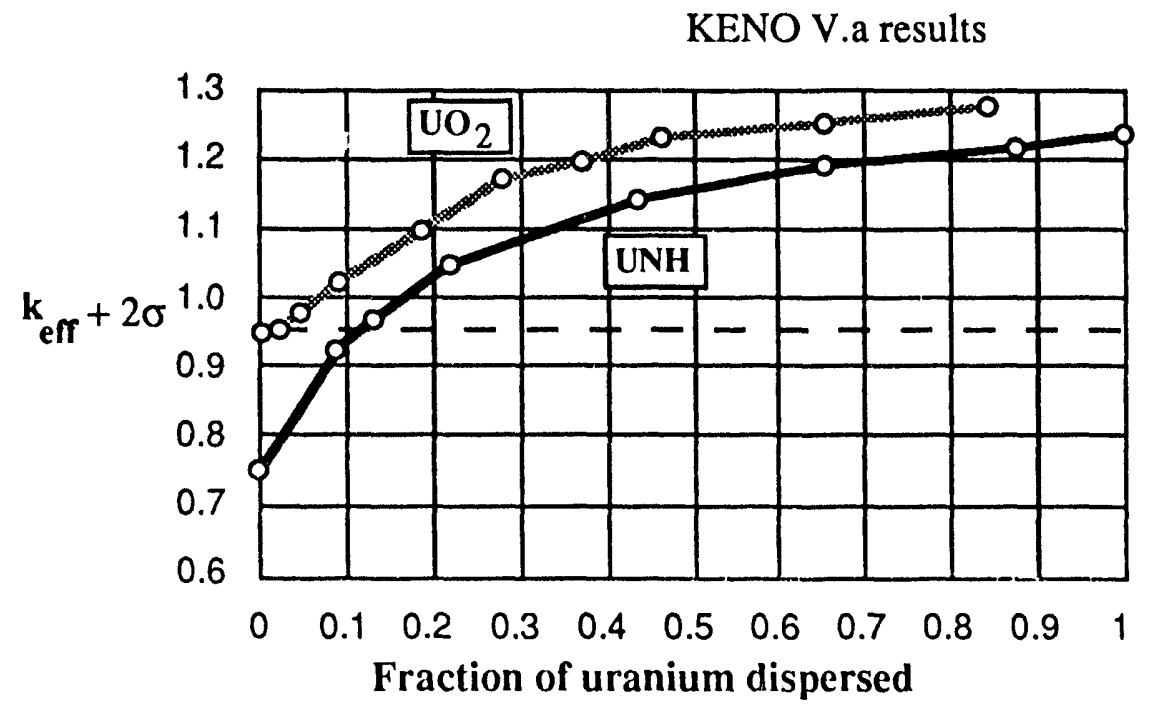

Fig. 8. KENO V.a calculations for receptacle and canister flooding with subsequent dispersal of the uranium from the 6 -inch container into the receptacle volume. The $235 \mathrm{U}$ mass is $8 \mathrm{~kg}$ in the $\mathrm{UO}_{2}$ cases and $5.56 \mathrm{~kg}$ in the UNH cases. The dashed line gives $\mathrm{k}_{\mathrm{eff}}=$ 0.95 .

\subsubsection{UF 6}

Although $\mathrm{UF}_{6}$ is stored in pressure-coded vessels and the chance of loss of moderation control is remote, there is a criticality potential if water should enter an 8-inch cylinder containing high-enriched $\mathrm{UF}_{6}$. A series of KENO V.a ${ }^{\mathrm{b}}$ calculations shows what could happen for various amounts of water admission to a single cylinder in a storage receptacle (stage 3 in Table 5). When water is admitted to $\mathrm{UF}_{6}$ a $\mathrm{UO}_{2} \mathrm{~F}_{2}$ hydrate or solution can form. In our model the $\mathrm{UO}_{2} \mathrm{~F}_{2}-\mathrm{H}_{2} \mathrm{O}$ mixture sits on top of dry $\mathrm{UF}_{6}$ (see Fig. 3). We considered it important to use the best data available for $\mathrm{UO}_{2} \mathrm{~F}_{2}-\mathrm{H}_{2} \mathrm{O}$ and thus did not use the KENO default densities, which are non-conservative for low $\mathrm{H} / \mathrm{U}$ values. We used density formulations reported in ORNL/CSD/TM-284, Appendix A. ${ }^{9}$, which show that the density of ${ }^{235} \mathrm{U}$ is higher in the hydrate than in the dry $\mathrm{UF}_{6}$ for low $\mathrm{H} / \mathrm{U}$ values. The material densities for the various cases are given in Appendix D, Table D9.

Fig. 9 shows the KENO results for a single UF$_{6}$-loaded 8 -inch cylinder in a waterflooded receptacle. The calculations were performed by inserting the volume of water into the cylinder as given by the abscissa and mixing an amount of $\mathrm{UF}_{6}$ with this water to give the required $\mathrm{H} / \mathrm{U}$. Criticality is definitely of concern when water is admitted into a cylinder. This result is not surprising, as the cylinder diameter of $20.4 \mathrm{~cm}$ exceeds the single-parameter

\footnotetext{
b The results of this section were obtained with a PC version of SCALE/KENO maintained by the LLNL Fission Energy and Systems Safety Program (FESSP). These replace calculations prepared before detailed drawings were obtained and used a cylinder wall thickness which was too thin and a uranium loading which was slightly too high. When this was noticed we no longer had access to the ORNL system. The PC calculations show that a small increase in water volume is needed to produce $k_{\text {eff }}=0.95$, mostly due to increased neutron absorption in the additional mass of monel in the new model.
} 
$\mathrm{UO}_{2} \mathrm{~F}_{2}$ solution limit of $13.7 \mathrm{~cm}$ given in American National Standard ANSLANS-8.1$1983^{1}$. We recommend that high-enriched $\mathrm{UF}_{6}$ be stored in cylinders which do not exceed the limit of ANSI/ANS-8.1-1983'.

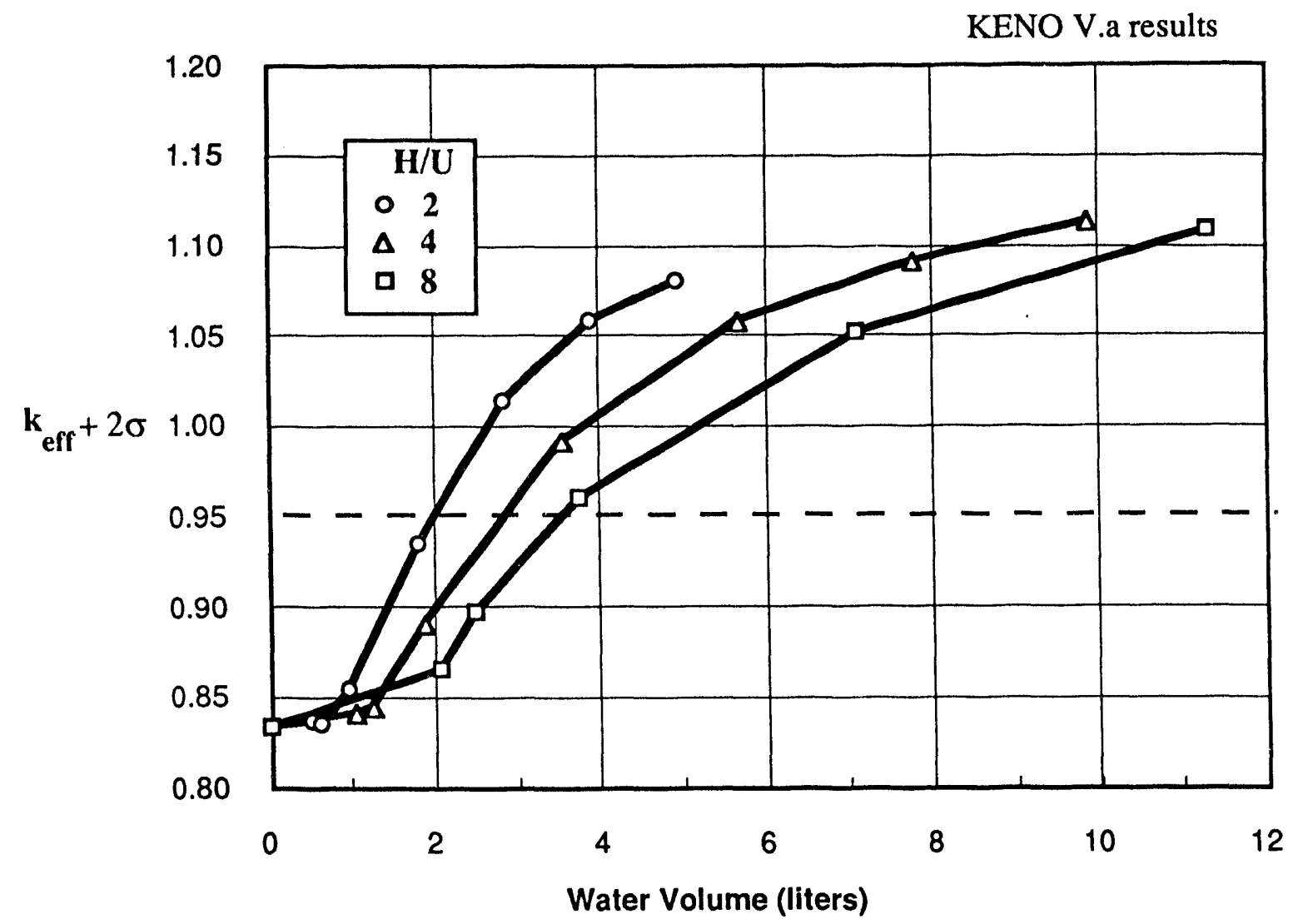

Fig. 9. Loss of moderation control for a single $\mathrm{UF}_{6}$-loaded cylinder in a water-flooded storage receptacle. The initial mass of $\mathrm{UF}_{6}$ is $255 \mathrm{lb}(115.67 \mathrm{~kg})$. Water is added to the top of the $\mathrm{UF}_{6}$, producing $\mathrm{UO}_{2} \mathrm{~F}_{2}{ }^{\bullet} \mathrm{H}_{2} \mathrm{O}$. The dashed line gives $\mathrm{k}_{\mathrm{eff}}=0.95$.

Loss of moderation control is a criticality safety concern even for $\mathrm{UF}_{6}$ cylinders in unflooded receptacles. In Fig. 10 we show the results of KENO V.a calculations for cases that are analogous to cases in Fig. 9, however there is no water in the receptacle space. These calculations show that criticality is possible with a little more water admitted into a cylinder. 
KENO V.a results

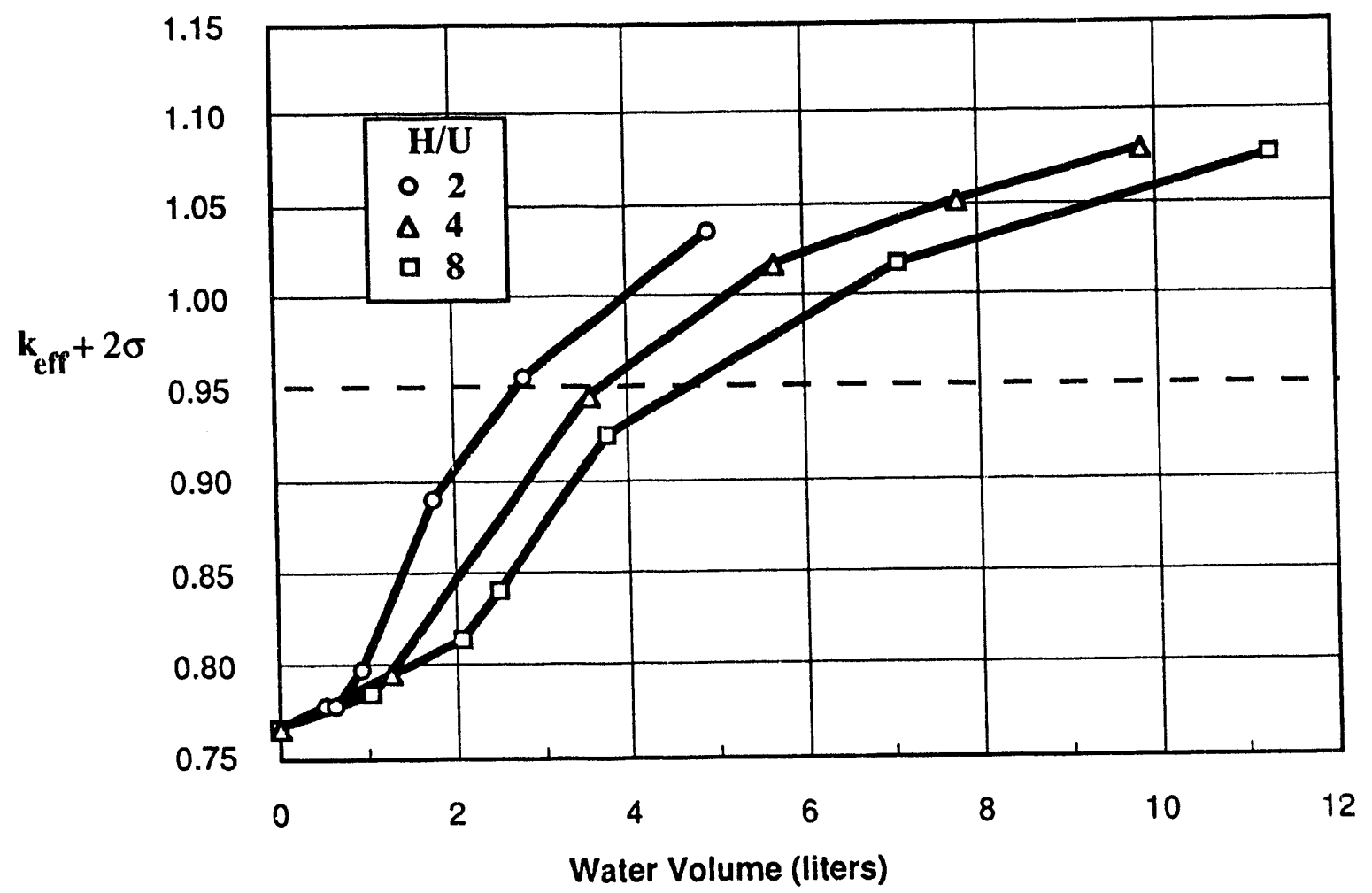

Fig. 10. Loss of moderation control for a single $U_{6}$-loaded cylinders in a dry storage receptacle. The initial mass of $\mathrm{UF}_{6}$ is $255 \mathrm{lb}(115.67 \mathrm{~kg})$. Water is added to the top of the $\mathrm{UF}_{6}$, producing $\mathrm{UO}_{2} \mathrm{~F}_{2} \bullet \mathrm{xH}_{2} \mathrm{O}$. The dashed line gives $\mathrm{k}_{\mathrm{eff}}=0.95$.

\subsection{Arrays}

\subsection{1 $\mathrm{UO}_{2}$}

In some vaults neutron interaction between units is important. The calculations of this study show that this is not the case for the HEU storage area. We compared results of calculations for isolated 6-inch diameter canisters with results for arrays of the same canisters inserted in unflooded underground storage receptacles. Table 8 shows the KENO results for various amounts of water mixed with the $\mathrm{UO}_{2}$ to give the $\mathrm{H} / \mathrm{U}$ values indicated. The differences between single units and arrays are very small. This is due to the large amount of isolating concrete between the receptacles. Since array interaction is not significant and the results reported in Sec. 8.1 show that unbreached single units (not exceeding mass or moderation limits) are subcritical, the storage array is also subcritical. 
Table 8. Results of KENO V.a calculations for $\mathrm{UO}_{2}{ }^{\mathrm{a}}$-loaded 6-inch canisters for various $\mathrm{H} / \mathrm{U}$ values.

\begin{tabular}{|l|c|c|c|}
\hline & \multicolumn{3}{|c|}{$k_{\text {eff }}+2 \sigma$} \\
\hline \hline$\left[\mathrm{H} / \mathrm{U} ; \text { 235 }^{\mathrm{U}} \operatorname{mass}(\mathrm{kg})\right]^{\mathrm{a}}:$ & $65 ; 2$ & $15 ; 5$ & $10 ; 8$ \\
\hline \hline Isolated units & 0.860 & 0.804 & 0.834 \\
\hline Infinite planar array & 0.871 & 0.827 & 0.845 \\
\hline
\end{tabular}

a The canisters contain $\mathrm{UO}_{2}$ at the mass limits described in Appendix $\mathrm{G}$. The materials are described in Table 1 and Appendix D, Table D1.

The density of uranium oxide can vary depending on how it is prepared, packed, or settles in storage and handling. We ran a series of KENO runs for infinite planar arrays containing $8 \mathrm{~kg} \mathrm{235} \mathrm{U}$ in which the density of dry $\mathrm{UO}_{2}$ varied from crystal density to the minimum permitted by the container size, i.e. the oxide distributed uniformly throughout the volume of the canister. Fig. 11 shows the results. The maximum $k_{\text {eff }}$ is for crystal density material, with no enhancement of reactivity resulting from density reduction.

KENO V.a results

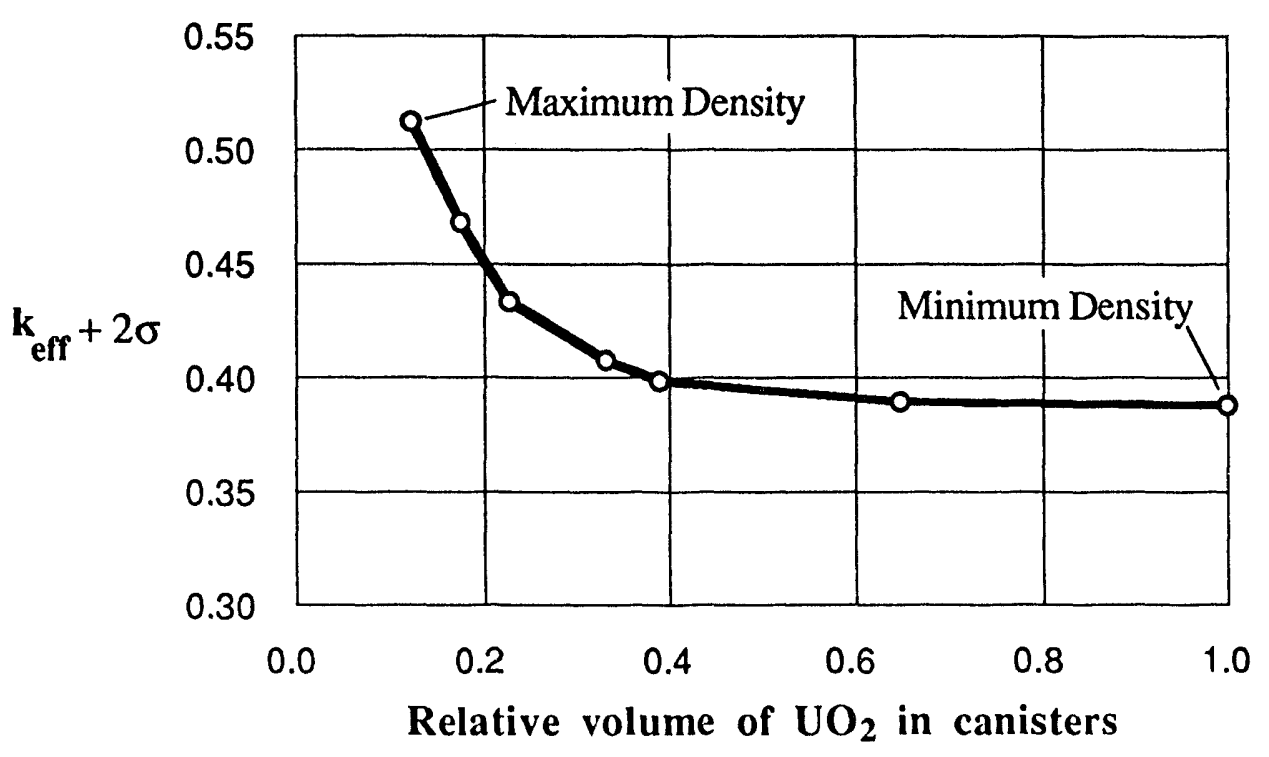

Fig. 11. Variation of $\mathrm{UO}_{2}$ density for an infinite planar array of 6-inch diameter canisters inside unflooded storage receptacles. Each canister is loaded with unmoderated $\mathrm{UO}_{2}$ containing $8 \mathrm{~kg}$ of ${ }^{235} \mathrm{U}$. The maximum density and minimum $\mathrm{UO}_{2}$ volume is for crystal density material. The minimum density is for complete filling of the canister. 


\subsubsection{UNH}

We also looked at uranyl nitrate hexahydrate (UNH) in polybottle containers inside the storage receptacle array. The material densities are given in Appendix D, Table D4. Table 9 shows results of calculations for infinite planar arrays. There is no concern for criticality even when water floods the receptacles unless the polybottle contents are dispersed into the larger receptacle volume, as seen in Section 8.1.3.

Table 9. Results of KENO V.a calculations for $\mathrm{UNH}^{\mathrm{a}}$-loaded polybottles in HEU storage receptacles. ${ }^{b}$

\begin{tabular}{|l|c|}
\hline \multicolumn{1}{|c|}{ Condition } & $\mathbf{k}_{\text {eff }}+\mathbf{2} \sigma$ \\
\hline \hline No receptacle flooding & 0.631 \\
\hline Receptacles flooded & 0.742 \\
\hline
\end{tabular}

a The polybottles are filled with UNH which is described in Table 1 and Appendix D, Table D4.

b An infinitc planar array model is used (see Fig. 2).

\subsubsection{Hydrogen Content in Concrete}

Array reactivity can be affected by the water content of the concrete. We simulated the effect of changes in the water content through a series of KENO calculations for an infinite array of 6-inch canisters with $8 \mathrm{~kg}{ }^{235} \mathrm{U}$ in $\mathrm{UO}_{2}-\mathrm{H}_{2} \mathrm{O}$ with $\mathrm{H} / \mathrm{U}=10$. The results are shown in Figure 12. An increase or decrease of the water content by a factor of two does not have a significant effect, however the unlikely scenario of complete removal can cause a noticeable increase in $k_{\text {eff. }}$.

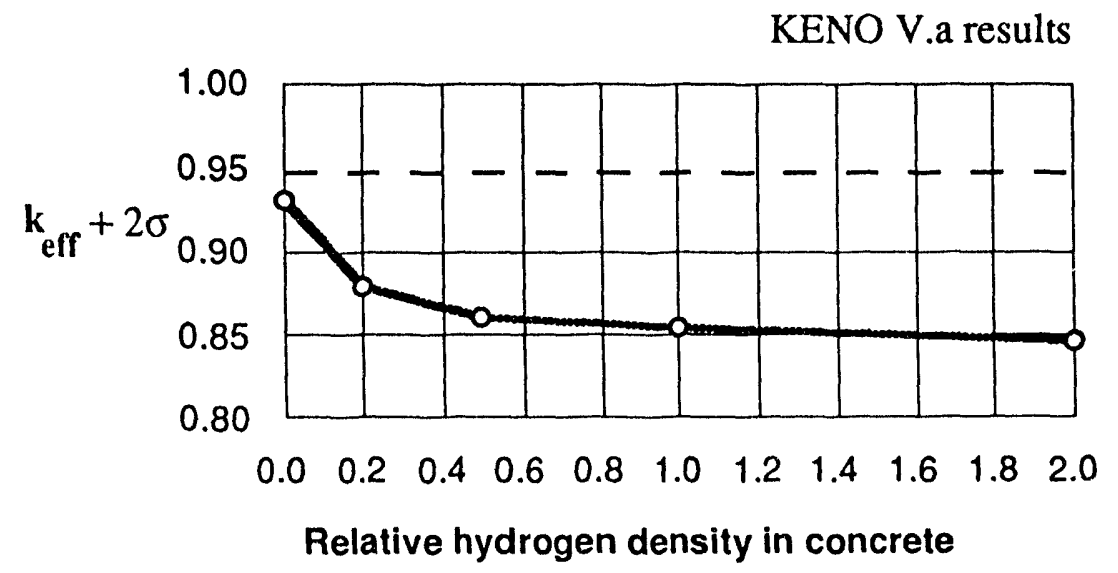

Fig. 12. Variation of hydrogen content of vault concrete for 6-inch-diameter canisters loaded with $\mathrm{UO}_{2}-\mathrm{H}_{2} \mathrm{O}$ inside dry storage receptacles. The hydrogen density is relative to the density as analyzed for the vault concrete. The mass of $235 \mathrm{U}$ is $8 \mathrm{~kg}$, with $\mathrm{H} / \mathrm{U}=10$. An infinite planar array is modeled (see Fig. 2). The dashed line gives $\mathrm{k}_{\mathrm{eff}}=0.95$. 


\section{Conclusions and Recommendations}

There are scenarios that could result in criticality in the HEU storage areas. These include loss of moderation control or exceeding uranium mass allowances. In addition to concern for criticality safety for units in storage receptacles, there is concern for the 6-inch diameter oxide canisters when they are handled outside of the below-ground storage receptacles or at ott.ur plant locations. A single over-mass unit or unit with additional moderator could possibly become critical when reflected by the equivalent of close-fitting water. In other words, double contingency safery is not assured for the 6-inch diameter canisters in the event of mass or moderation in excess of the current limits (cf. Figs. 5 and 6).

This criticality potential for oxide storage can be diminished, however, if 5-inch, or smaller, diameter containers are used. This change would help to insure compliance with current standards and DOE orders, e.g. DOE 5480.2411, Section $7 \mathrm{a}(2)$, requires:

4.2.3 Geometry Control. As a first priority, reliance shall be placed on equipment design in which dimensions of the contained fissionable material and spacing between equipment are limited via passive engineering controls...

Even with smaller diameter containers, there still is a potential for criticality if a receptacle containing an open or breached container is flooded. The likelihood of flooding and breaching is being investigated by a separate probabilistic analysis. Hovever, the potential can be eliminated by minimizing the possible admission of water into receptacles by filling the receptacle void space with a non-porous, filler material, such as a sleeve with an appropriate diameter hole to receive a canister. In addition, the use of more nearly "leak-proof" container materials is recommended, as has been suggested in earlier analyses. 10

This study has also shown that the UF6 canisters with high-enriched uranium will be a criticality hazard if there is loss of moderation control. Criticality safety thus depends upon maintaining the $\mathrm{UF}_{6}$ in a dry condition. In order to ensure subcriticality if moderation control is not maintained, we recommend that high-enriched $\mathrm{UF}_{6}$ be stored in cylinders with diameters which do not exceed the single-parameter limit $(13.7 \mathrm{~cm})$ of Table 1 of ANSI/ANS8.1-1983.1 


\section{References}

1. American National Standard for Nuclear Criticality Safety in Operations with Fissionable Material Outside Reactors, ANSI/ANS 8.1, American Nuclear Society, LaGrange Park, Illinois, October 7, 1983.

2. Lutz, H. F., Activity Plan for X-345 Sub Task, memo to Tom Lemons, GDPNCS-92-05, March 6, 1992.

3. Lee, Billy L. Jr., Validation of Nuclear Criticality Software, KENO V.s., with the 27 Group ENDF/B-IV Cross Sections, Portsmouth Gaseous Diffusion Plant, Piketown, OH, POEF-T-3636 (to be published).

4. Portsmouth Gaseous jiffusion Plant Final Safety Analysis Report, Volume 1-3, GAT/GDP-1073, April, 1985.

5. Tayloe, R. W., Summary of Nuclear Criticality Safety Rules for X-345 Storage Building, Goodyear Atomic Corporation, GAT-DM-1444, Rev. 1, April 10, 1984.

6. Newvahner, R. L., Nuclear Criticality Safety Analysis of X-345 Storage Building, Martin Marietta Energy Systems, Inc., POEF-T-3489, September 30, 1988.

7. Carter, R. D., Kiel, G. R., and Ridgway, K. R., Criticality Handbook, Vol. II, Atlantic Richfield Hanford Company, ARH-600, Vol. II, May 23, 1969.

8. Gmelin, Handbook of Inorganic Chemistry, 8th Ed., Supplement Volume C7, Nitrogen Compounds, Springer-Verlag, Berlin, 1981.

9. W. C. Jordan, and J. C. Turner, Minimum Mass of Moderator Required for Criticality of Homogeneous Low-Enriched Uranium Systems, Martin Marietta Energy Systems, Inc., ORNL/CSD/TM-284, December, 1992.

10. Rumble, Bonnie J., Water Intrusion into X-345 Vaults, Martin Marietta Energy Systems, Inc., POEF-T-3447, March 3, 1987.

11. Nuclear Criticality Safety, DOE Order 5480.24, U.S. Department of Energy, Washington, D.C., August 12, 1992.

12. Oxenham, R. L., Netzer, W. D., and Miller, R. R., Interim Moderation Control Limits for Oxides in X-345, Martin Marietta Energy Systems, Portsmouth, POEF-DM-1554, March 10, 1988.

13. D. A. Shisler, Martin Marietta Energy Systems, Portsmouth, Nuclear Materials Control, internal correspondence 504-87-04, January 9, 1987. 


\section{Documentation on X-345 Criticality Calculations}

The following letters and reports document the calculational efforts to set the design limits and administrative controls in X-345:

\begin{tabular}{|c|c|c|c|}
\hline Author & Date & Report Number & Title \\
\hline Feuerbacher, J. L. & $2 / 21 / 69$ & GAT-DM-1128 & $\begin{array}{l}\text { A Nuclear Safety Guide for Handling, Storing and } \\
\text { Recovering Uranium Metal Slugs, Pellets and } \\
\text { Shavings, and High Density Urarium Compounds }\end{array}$ \\
\hline Feuerbacher, J. L. & $11 / 1 / 74$ & GAT-532-74-187 & Containers of Uranium Oxide in Special Vaults \\
\hline Feuerbacher, J. L. & $5 / 11 / 76$ & GAT-532-76-143 & $\begin{array}{l}\text { Safe Fill of Uranium Oxide in 5" (ID) or 6" (ID) Cans } \\
\text { at Limited Moderation, for All Enrichments }\end{array}$ \\
\hline Feuerbacher, J. L. & $6 / 8 / 76$ & GAT-532-76-184 & $\begin{array}{l}\text { Nuclear Safety of Storing 5.24" ID and 6-3/16" ID } \\
\text { Cans of Uranium Oxide at Limited Moderation in a } \\
\text { Proposed Vault Layout }\end{array}$ \\
\hline $\begin{array}{l}\text { Feuerbacher, J. L. } \\
\text { and K. R. Yates }\end{array}$ & $11 / 17 / 76$ & GAT-DM-1345 & $\begin{array}{l}\text { Storing } 5 ", 8 " \text { and } 12 " \text { UF6 Cylinders in Vaults } \\
\text { Under the Concrete Floor of a Storage Building }\end{array}$ \\
\hline Oxenham, R. L. & $10 / 3 / 77$ & GAT-530-77-73 & $\begin{array}{l}\text { The Approval of Drawings of Proposed In-Floor } \\
\text { Storage Arrangements for Future X-345 Facility }\end{array}$ \\
\hline Feuerbacher, J. L. & $12 / 14 / 77$ & GAT-530-77-97 & Studies of Most Recent Design for X-345 \\
\hline $\begin{array}{l}\text { Feuerbacher, J. L. } \\
\text { and K. R. Yates }\end{array}$ & $1 / 18 / 77$ & $\begin{array}{l}\text { GAT-DM-1354, } \\
\text { Addendum } 1\end{array}$ & $\begin{array}{l}\text { Storing } 5 ", 8 " \text { and } 12 " \text { UF } 6 \text { Cylinders in Vaults } \\
\text { Under the Concrete Floor of a Storage Building - } \\
\text { Multiplication Factors of Single 8" and } 12 \text { " Cylinders } \\
\text { Reflected by Concrete, }\end{array}$ \\
\hline $\begin{array}{l}\text { Feuerbacher, J. L. } \\
\text { and K. R. Yates }\end{array}$ & $2 / 10 / 77$ & GAT-DM-1358 & $\begin{array}{l}\text { Storing 5" ID x 48" Polybottles of Uranium } \\
\text { Solution, 5" ID x } 911 \text { Plastic Bottles of UNH, and 4" } \\
\text { ID } \times 9-1 / 2 \text { " Polybottles of Uranium Oxide in Vaults } \\
\text { Under the Concrete Floor of a Storage Building }\end{array}$ \\
\hline Yates, K. R. & $3 / 16 / 77$ & $\begin{array}{l}\text { GAT-DM-1358, } \\
\text { Addendum } 1\end{array}$ & $\begin{array}{l}\text { The Consequence of Spilling the Contents of a } 5 \text { " ID } \\
\text { x } 48 \text { " Polybottle into Open (or Leaking) Vaults } \\
\text { (Receptacles) Containing 5" ID x 48" Polybottles or } \\
5 \text { " ID UF6 Cylinders }\end{array}$ \\
\hline $\begin{array}{l}\text { Feuerbacher, J. L. } \\
\text { and K. R. Yates }\end{array}$ & $4 / 5 / 77$ & $\begin{array}{l}\text { GAT-DM-1354, } \\
\text { Addendum } 2\end{array}$ & $\begin{array}{l}\text { Storing } 5 ", 8 " \text { and } 12 " \text { UF } 6 \text { Cylinders in Vaults } \\
\text { Under the Concrete Floor of a Storage Building - } \\
\text { Between Concrete Slabs for } 5 \text { " Cylinders }\end{array}$ \\
\hline $\begin{array}{l}\text { Feuerbacher, J. L. } \\
\text { and K. R. Yates }\end{array}$ & $5 / 9 / 77$ & $\begin{array}{l}\text { GAT-DM-1358, } \\
\text { Addendum } 2\end{array}$ & $\begin{array}{l}6 " \text { ID } \times 15^{\prime \prime} \text { Cans of Oxide at H/U Between } 0.4 \text { and } \\
15.0 \text { in the New SNM Building }\end{array}$ \\
\hline Feuerbacher, J. L. & $8 / 11 / 80$ & $\begin{array}{l}\text { GAT-DM-1358, } \\
\text { Addendum } 3\end{array}$ & $\begin{array}{l}\text { Storing 5" ID x 48" Polybottles of Uranium } \\
\text { Solution, 5" ID x 9" Plastic Bottles of UNH, and 4" } \\
\text { ID } \times 9-1 / 2 " \text { Polybottles of Uranium Oxide in Vaults } \\
\text { Under the Concrete Floor of a Storage Building }\end{array}$ \\
\hline Feuerbacher, J. L. & $9 / 19 / 80$ & $\begin{array}{l}\text { GAT-DM-1358, } \\
\text { Addendum } 4\end{array}$ & $\begin{array}{l}\text { Storing 5" ID x 48" Polybottles of Uranium } \\
\text { Solution, } 5 \text { ID } \times 9 \text { 9" Plastic Bottles of UNH, and 4" } \\
\text { ID x 9-1/2" Polybottles of Uranium Oxide in Vaults } \\
\text { Under the Concrete Floor of a Storage Building }\end{array}$ \\
\hline Feuerbacher, J. L. & $2 / 20 / 80$ & $\begin{array}{l}\text { GAT-DM-1444, } \\
\text { Appendices I and II }\end{array}$ & $\begin{array}{l}\text { Summary of Nuclear Criticality Safety Rules for X- } \\
345 \text { Storage Building }\end{array}$ \\
\hline $\begin{array}{l}\text { Shively, D. M. and } \\
\text { R. W. Tayloe }\end{array}$ & $5 / 16 / 83$ & GAT-T-3234, & $\begin{array}{l}\text { Criticality Calculations for Flooded Oxide Containers } \\
\text { in X-345 }\end{array}$ \\
\hline
\end{tabular}




\begin{tabular}{|l|l|l|l|}
\hline Newvahner, R. L. & $9 / 30 / 88$ & POEF-T-3489 & $\begin{array}{l}\text { Nuclear Criticality Safety Analysis of X-345 Uranium } \\
\text { Solution Storage Incident }\end{array}$ \\
\hline Tayloe, R. W. & $4 / 10 / 84$ & $\begin{array}{l}\text { GAT-DM-1444, } \\
\text { Rev. 1 }\end{array}$ & $\begin{array}{l}\text { Summary of Nuclear Criticality Safety Rules for X- } \\
\text { 345 Storage Building }\end{array}$ \\
\hline Rumble, Bonnie J. & $3 / 9 / 87$ & POEF-T-3447 & Water Intrusion into X-345 Vaults \\
\hline
\end{tabular}




\section{APPENDIX B \\ KENO V.a / SCALE Job Control Description}

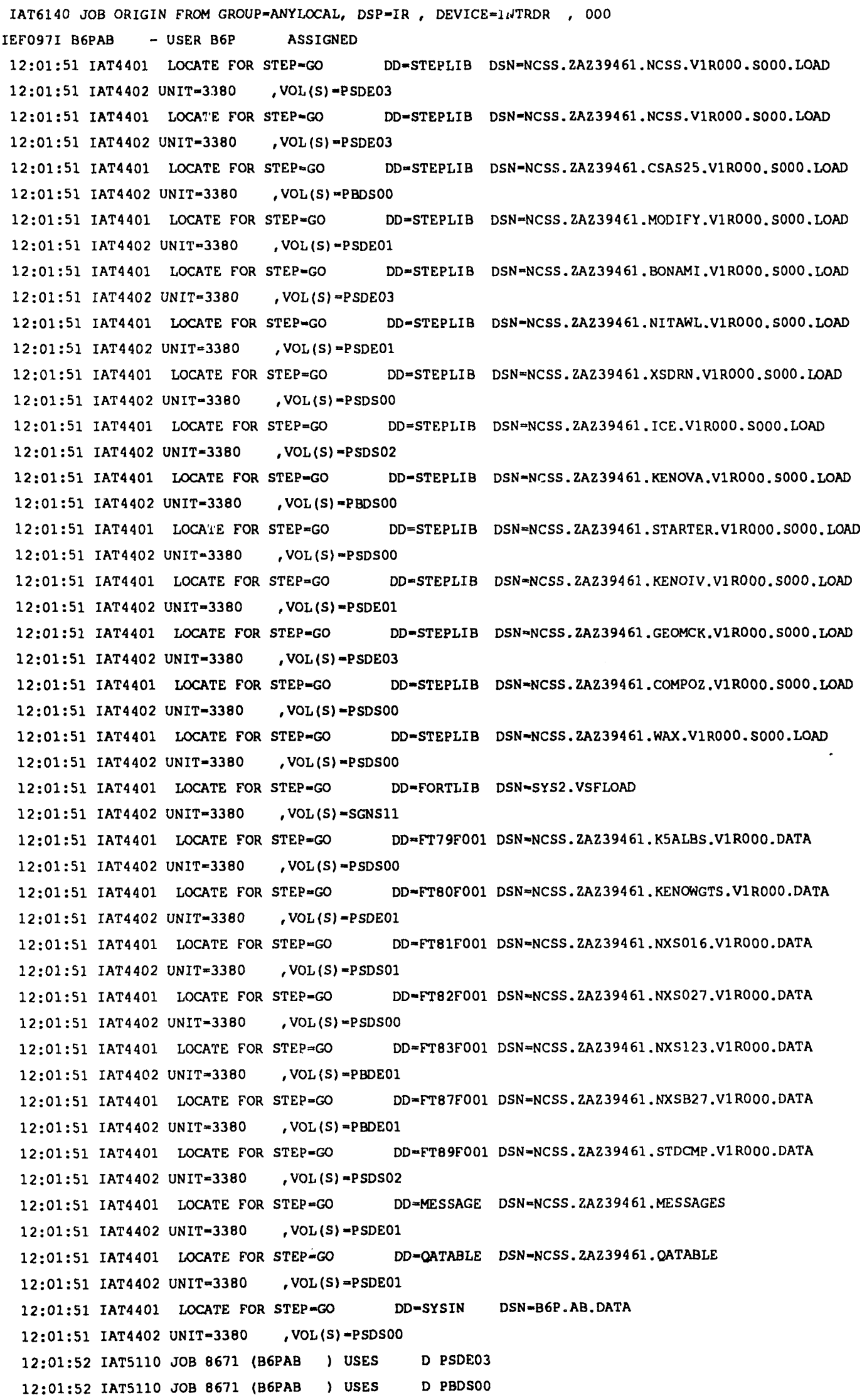




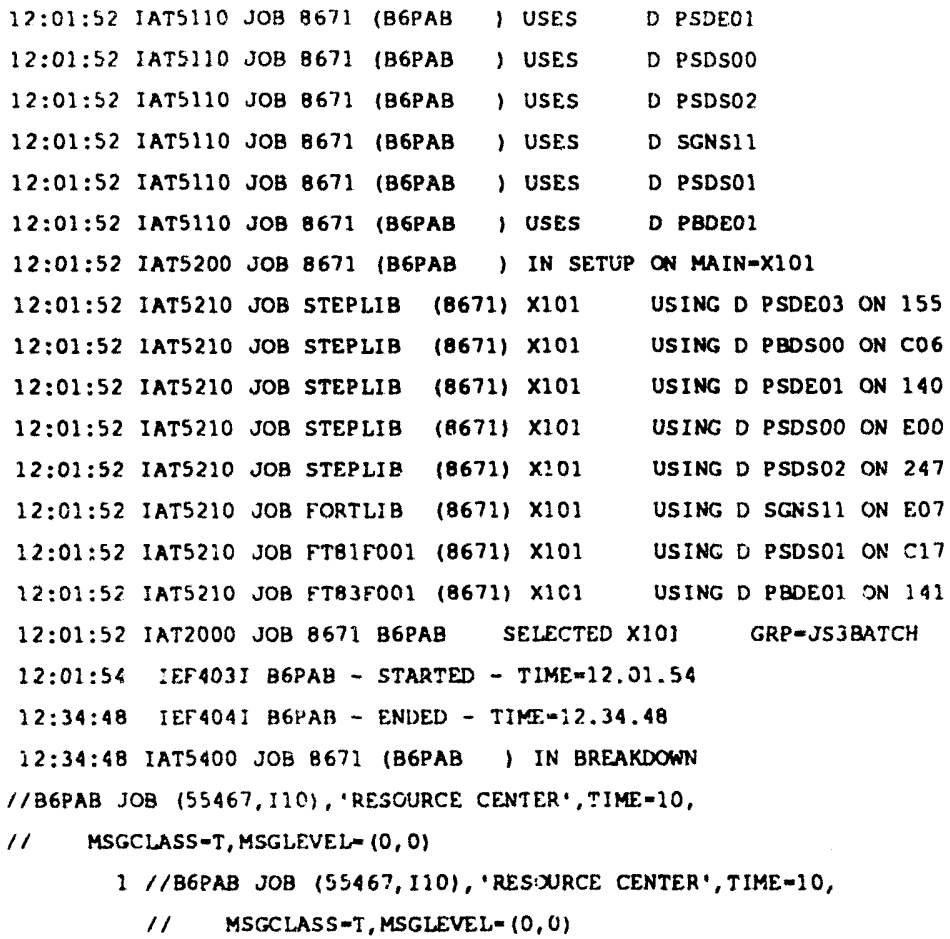

IEF3741 STEP /GO , STOP 93032.1234 CPU SMIN 33.62SEC SRB OMIN 02.01SEC VIRT 2712K SYS 216K EXT

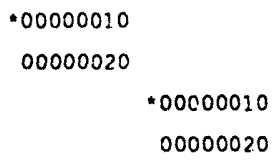


-KXY0002 FT16FC01 CO2 SYS93032.T120148.RA000.B6PAB.R0000015

"KXY0002 FT17F001 C02 SYS93032.T120148.RA000.B6PAB.R0000016

*KXY0002 FT18F001 900 SYS93032.T120148.RA000.B6PAB.R0000017

*KXY0002 FT19F001900 SYS93032.T120148.RA000.B6PAB. R0000018

*KXY0002 FT79F001 E00 NCSS.zAZ39461.K5ALBS.V1R000.DATA

"KXY0002 FT8OFO01 140 NCSS.ZAZ39461.KENOWGTS.V1ROOO.DATA

-KXY0002 FT81F001 C17 NCSS.ZAZ39461.NXS016.V1R000.DATA

.KXY0002 FT82F001 E00 NCSS.ZAZ39461.NXS027.V1R000.DATA

"KXY0002 FT83F001 141 NCSS.2A239461.NXS123.VIROOO.DATA

*KXY0002 FT87F001 141 NCSS.2A239461.NXSB27.V1ROOO.DATA

"KXY0002 FT89F001 247 NCSS.ZAZ39461.STDCMP.V1ROOO.DATA

*KXY0002 FT90F001 900 SYS93032.T120148.RA000.B6PAB.R0000019

*KXY0002 FT91F001 900 SYS93032.T120148.RA000.B6PAB.R0000020

*KXY0002 FT92F001 C02 SYS93032.T120148.RA000.B6PAB.RO00D021

-KXY0002 FT93F001 C02 SYS93032.T120148.RA000.B6PAB.R0000022

-KXY0002 FT94F001 C02 SYS93032.T120148.RA000.B6PAB.R0000023

"KXY0002 ET95F001 C02 SYS93032.T120148.RA000.B6PAB.RO000024

*KXY0002 FT96FO01 C02 SYS93032.T120148.RA000.B6PAB.R0000025

*KXY0002 FT97F001 C02 SYS93032.T120148.RA000.B6PAB.R00000'6

*KXY0002 FT98F001 C02 SYS93032.T120148.RA000.B6PAB, R0000027

"KXY0002 MESSAGE 140 NCSS.ZAZ39461.MESSAGES

"KXY0002 QATABLE 140 NCSS.2Az39461.QATABLE

-KXY0002 SYSIN EOO B6P.AB.DATA

-KXY0003 VIRTUAL STORAgE USED $1712 \mathrm{~K}$

TOTAL EXCP COUNT FOR STEP

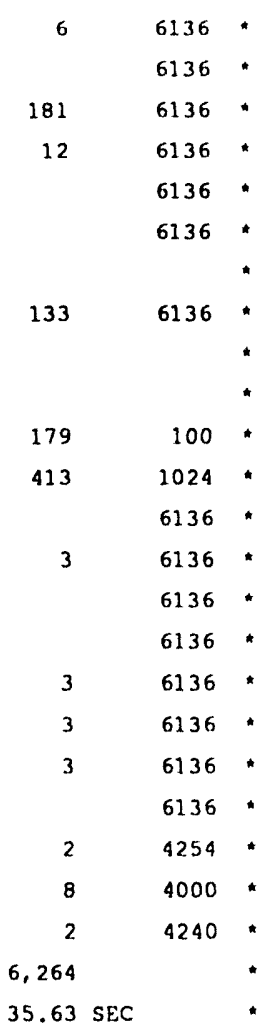

*KXY0004 O1 FEB $93.03212 .34 .48 .54 \quad$ CPU TIME FOR STEP 0005 MIN 35.63 SEC

IEF375I JOB /BGPAB / START 93032.1201

IEF376I JOB /B6PAB / STOP $93032.2234 \mathrm{CPU}$ 5MIN 33.62SEC SRB OMIN 02.01SEC

"KXY0003
"KXY0006
KXY0006

1 PRIMARY MODULE ACCESS AND INPUT RECORD ( NCSS DRIVER - 07/24/91 - 15.25)

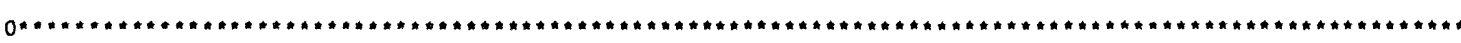

Messages of current interest to NCSS users will appear here

NCSS

$2 / 26 / 92$

This is the QA version of the Martin Marletta Energy Systems crlticality safety software. It is now * avallable for valldation and production use.

.

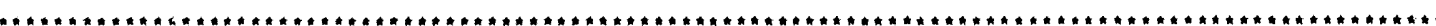

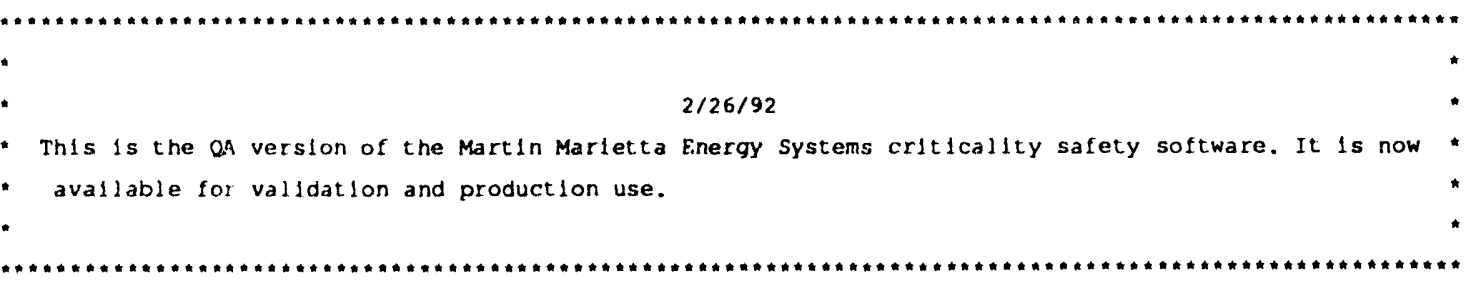




\section{Appendix $\mathrm{C}$ \\ Confirmatory Calculations with MCNP}

A number of MCNP Monte Carlo neutron transport code calculations were done by an analyst who was not involved in setting up the KENO input, providing a degree of independent review. Also, since MCNP has been developed completely independently of KENO and employs very different Monte Carlo algorithms and cross section treatment (pointwise) we have additional assurance in the validity of the KENO results of this study. Table $\mathrm{C} 1$ shows close agreement between MCNP and KENO for comparable situations. For these studies, we used a cell depicted in Fig. 4-2 of POEF-3447 10 , which considered the receptacle to be off-center in a rectangular box in an effort to provide a conservative model for array calculations.

Table C1. Results of MCNP and KENO V.a calculations for $\mathrm{UO}_{2}{ }^{\mathrm{a}}$ in uranium oxide/UNH storage area.

\begin{tabular}{|c|c|c|c|}
\hline \multirow{2}{*}{ Array } & \multirow{2}{*}{$\begin{array}{c}\text { Receptacle } \\
\text { Flooding }\end{array}$} & \multicolumn{2}{|c|}{$\mathrm{k}_{\text {eff }}$} \\
\cline { 3 - 4 } & & MCNP & KENO \\
\hline \hline $1 \times 1 \times 1$ & No & $0.857 \pm .003$ & $0.867 \pm .005$ \\
\hline $1 \times 1 \times 1$ & Yes & $0.961 \pm .003$ & $0.988 \pm .005$ \\
\hline $15 \times 15 \times 1$ & Yes & $1.030 \pm .002$ & $1.021 \pm .005$ \\
\hline
\end{tabular}

a Each canister holds $8 \mathrm{~kg} 235 \mathrm{U}$ with $\mathrm{H} / \mathrm{U}=15(8 \mathrm{~kg}$ was the $\mathrm{H} / \mathrm{U}=15$ loading limit until the memo referenced in Appendix $G$ lowered the limit to $5 \mathrm{~kg}$ ).

We also compared MCNP and KENO calculations for UNH, with a level of hydration of 6 , which is doubly batched inside the receptacle, with no container breaching or water flooding of the receptacle. Input data and results are provided in Table C2.

Table C2. Results of MCNP calculations for UNH in uranium oxide/UNH storage area.

\begin{tabular}{|c|c|c|c|c|}
\hline \multicolumn{2}{|c|}{} & \multicolumn{2}{c|}{$k_{\text {eff }}$} \\
\hline \hline Compound & Density $\left(\mathrm{g} / \mathrm{cm}^{3}\right)$ & Array & MCNP & KENO \\
\hline $\mathrm{UO}_{2}\left(\mathrm{NO}_{3}\right)_{2} \cdot 6 \mathrm{H}_{2} \mathrm{O}$ & $2.807^{\mathrm{a}}$ & $\infty \times \infty \times 1$ & $0.8169 \pm .0035$ & $0.787 \pm .005$ \\
\hline
\end{tabular}

aThe crystalline density used here is somewhat higher than for loose powders which are actually stored. The density is from Reference 7, pp II.D.2-1. 


\section{Appendix D}

Computer Input Spreadsheets

for Uranium Oxides \& UNH

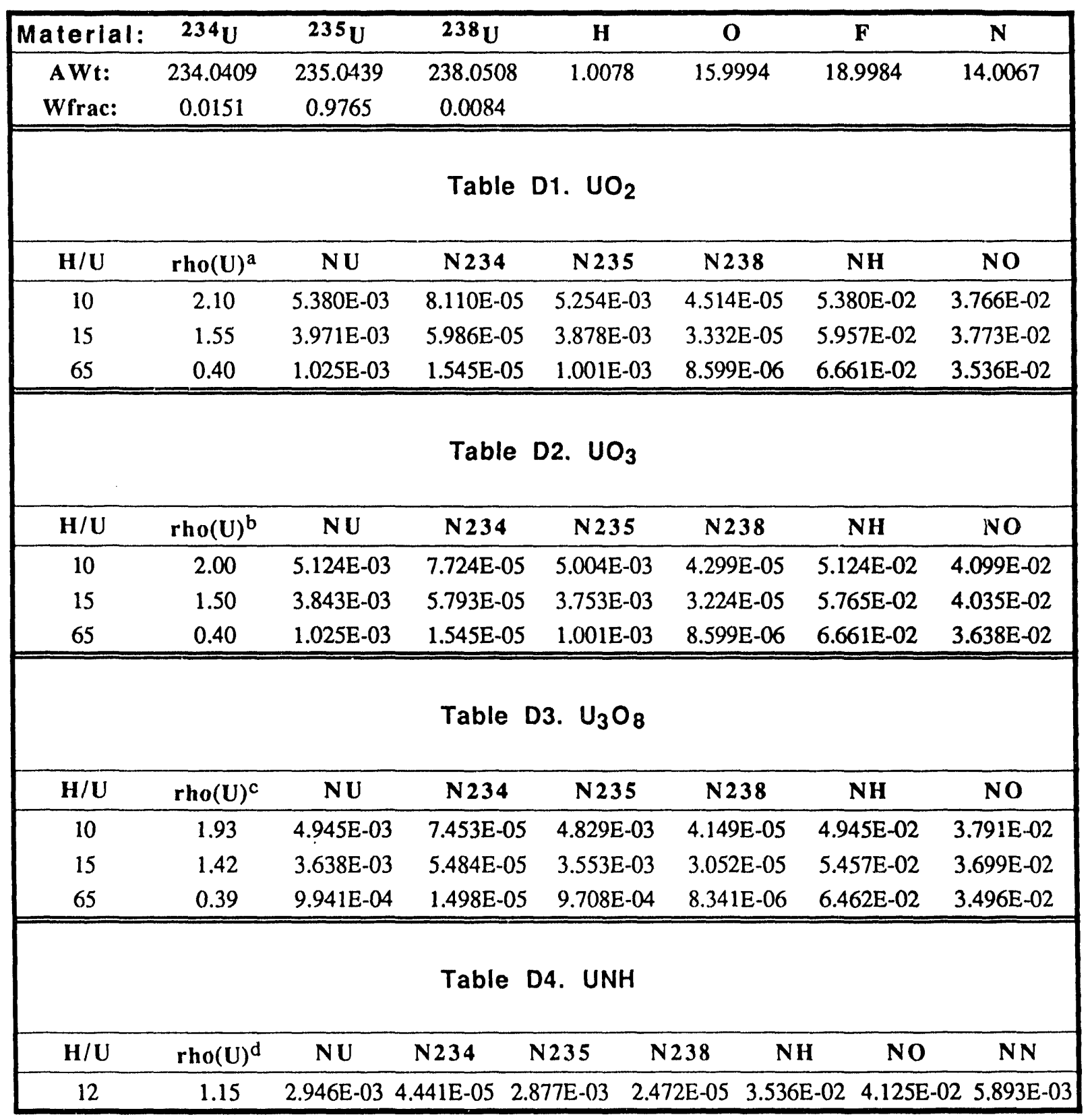

a Densities are from ARH-600, Page III.B.2-2

b Densities are from ARH-600, Page III.B.2-2

c Densities are determined using ORNL/CSD/TM-284, Appendix A, Eq. A.1.

d Densities are from ARH-600, Page III.B.2-4 
Table D5. Computer Input Spreadsheet Data for 6-inch Canisters with Admission of Water

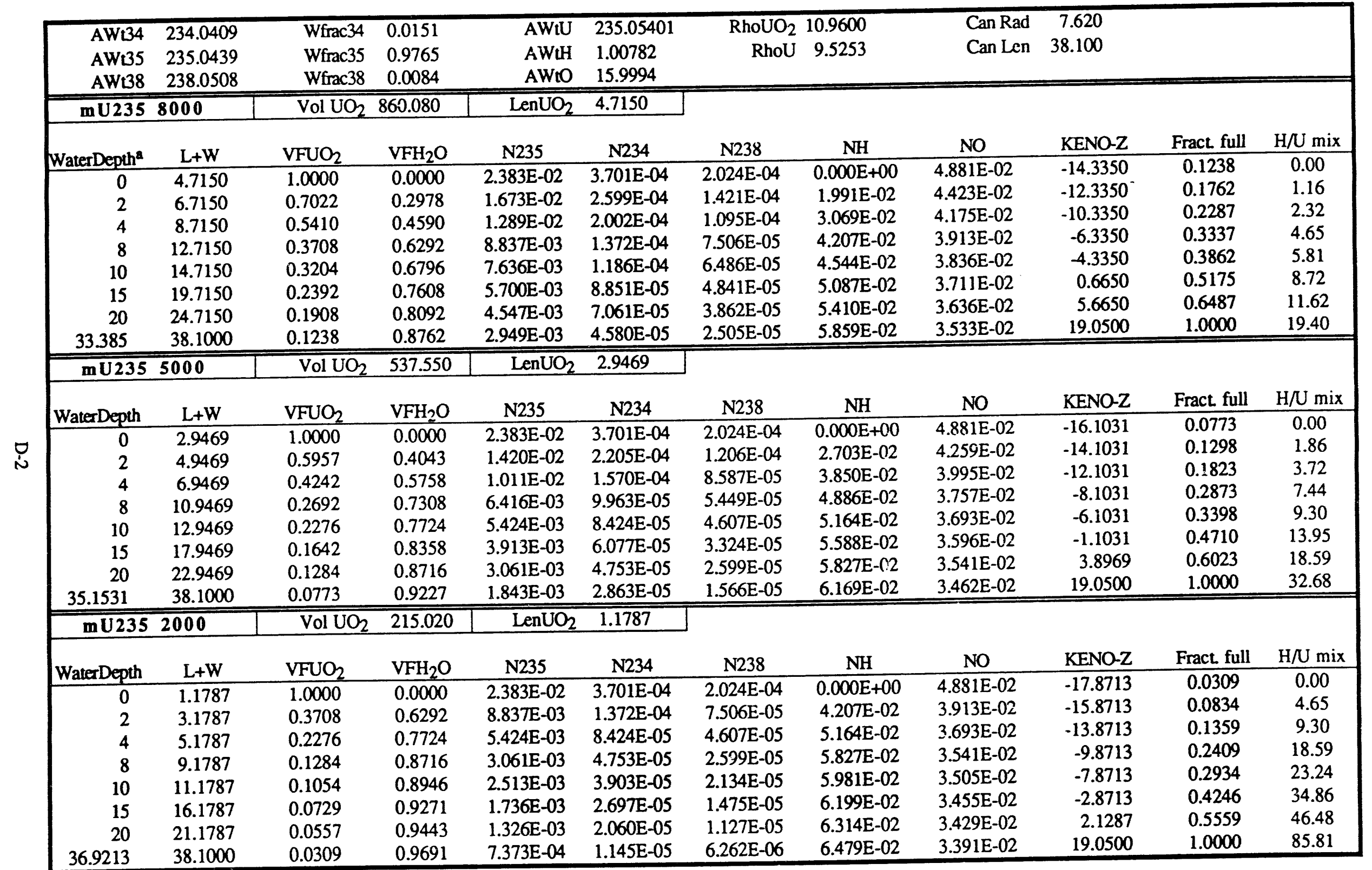

a Water is added to the top of the $\mathrm{UO}_{2}$ to the indicated depth and then mixed with the $\mathrm{UO}_{2}$. 
Table D6. Computer Input Spreadsheet Data for 5-inch Canisters with Admission of Water

\begin{tabular}{|c|c|c|c|c|c|c|c|c|c|c|c|}
\hline $\begin{array}{l}\text { AWt34 } \\
\text { AWt35 } \\
\text { AWt38 } \\
\end{array}$ & $\begin{array}{l}234.0409 \\
235.0439 \\
238.0508 \\
\end{array}$ & $\begin{array}{l}\text { Wfrac34 } \\
\text { Wfrac35 } \\
\text { Wfrac38 } \\
\end{array}$ & $\begin{array}{l}0.0151 \\
0.9765 \\
0.0084 \\
\end{array}$ & $\begin{array}{l}\text { AWtU } \\
\text { AWtH } \\
\text { AWtO } \\
\end{array}$ & $\begin{array}{l}235.05401 \\
1.00782 \\
15.9994 \\
\end{array}$ & $\begin{array}{r}\mathrm{RhoUO}_{2} \\
\text { RhoU }\end{array}$ & $\begin{array}{r}10.9600 \\
9.5253\end{array}$ & $\begin{array}{l}\text { Can Rad } \\
\text { Can Len }\end{array}$ & $\begin{array}{r}6.350 \\
38.100\end{array}$ & & \\
\hline WaterDepth ${ }^{\mathrm{a}}$ & $\mathrm{L}+\mathrm{W}$ & $\mathrm{VFUO}_{2}$ & $\mathrm{VFH}_{2} \mathrm{O}$ & N235 & N234 & N238 & $\mathrm{NH}$ & NO & KENO-Z & Fract. full & $\mathrm{H} / \mathrm{U} \operatorname{mix}$ \\
\hline $\begin{array}{r}0 \\
2 \\
4 \\
8 \\
10 \\
15 \\
20 \\
31.3107 \\
\end{array}$ & $\begin{array}{c}6.7896 \\
8.7896 \\
10.7896 \\
14.7896 \\
16.7896 \\
21.7896 \\
26.7896 \\
38.1000 \\
\end{array}$ & $\begin{array}{l}1.0000 \\
0.7725 \\
0.6293 \\
0.4591 \\
0.4044 \\
0.3116 \\
0.2534 \\
0.1782 \\
\end{array}$ & $\begin{array}{l}0.0000 \\
0.2275 \\
0.3707 \\
0.5409 \\
0.5956 \\
0.6884 \\
0.7466 \\
0.8218 \\
\end{array}$ & $\begin{array}{l}2.383 \mathrm{E}-02 \\
1.841 \mathrm{E}-02 \\
1.500 \mathrm{E}-02 \\
1.094 \mathrm{E}-02 \\
9.638 \mathrm{E}-03 \\
7.426 \mathrm{E}-03 \\
6.040 \mathrm{E}-03 \\
4.247 \mathrm{E}-03 \\
\end{array}$ & $\begin{array}{l}3.701 \mathrm{E}-04 \\
2.859 \mathrm{E}-04 \\
2.329 \mathrm{E}-04 \\
1.699 \mathrm{E}-04 \\
1.497 \mathrm{E}-04 \\
1.153 \mathrm{E}-04 \\
9.380 \mathrm{E}-05 \\
6.583 \mathrm{E}-05 \\
\end{array}$ & $\begin{array}{l}2.024 \mathrm{E}-04 \\
1.564 \mathrm{E}-04 \\
1.274 \mathrm{E}-04 \\
9.293 \mathrm{E}-05 \\
8.186 \mathrm{E}-05 \\
6.307 \mathrm{E}-05 \\
5.130 \mathrm{E}-05 \\
3.603 \mathrm{E}-05 \\
\end{array}$ & $\begin{array}{c}0.000 \mathrm{E}+00 \\
1.521 \mathrm{E}-02 \\
2.479 \mathrm{E}-02 \\
3.617 \mathrm{E}-02 \\
3.982 \mathrm{E}-02 \\
4.603 \mathrm{E}-02 \\
4.991 \mathrm{E}-02 \\
5.495 \mathrm{E}-02 \\
\end{array}$ & $\begin{array}{l}4.881 \mathrm{E}-02 \\
4.531 \mathrm{E}-02 \\
4.311 \mathrm{E}-02 \\
4.049 \mathrm{E}-02 \\
3.965 \mathrm{E}-02 \\
3.822 \mathrm{E}-02 \\
3.733 \mathrm{E}-02 \\
3.617 \mathrm{E}-02 \\
\end{array}$ & $\begin{array}{r}-12.2604 \\
-10.2604 \\
-8.2604 \\
-4.2604 \\
-2.2604 \\
2.7396 \\
7.7396 \\
19.0500 \\
\end{array}$ & $\begin{array}{l}0.1782 \\
0.2307 \\
0.2832 \\
0.3882 \\
0.4407 \\
0.5719 \\
0.7031 \\
1.0000 \\
\end{array}$ & $\begin{array}{r}0.00 \\
0.81 \\
1.61 \\
3.23 \\
4.04 \\
6.05 \\
8.07 \\
12.63 \\
\end{array}$ \\
\hline WaterDepth & $\mathrm{L}+\mathrm{W}$ & $\mathrm{VFUO}_{2}$ & $\mathrm{VFH}_{2} \mathrm{O}$ & N235 & N234 & N238 & NH & NO & KENO-Z & Fract. full & $\mathrm{H} / \mathrm{U}$ mix \\
\hline $\begin{array}{r}0 \\
2 \\
4 \\
8 \\
10 \\
15 \\
20 \\
33.8567 \\
\end{array}$ & $\begin{array}{c}4.2435 \\
6.2435 \\
8.2435 \\
12.2435 \\
14.2435 \\
19.2435 \\
24.2435 \\
38.1000 \\
\end{array}$ & $\begin{array}{l}1.0000 \\
0.6797 \\
0.5148 \\
0.3466 \\
0.2979 \\
0.2205 \\
0.1750 \\
0.1114 \\
\end{array}$ & $\begin{array}{l}0.0000 \\
0.3203 \\
0.4852 \\
0.6534 \\
0.7021 \\
0.7795 \\
0.8250 \\
0.8886 \\
\end{array}$ & $\begin{array}{l}2.383 \mathrm{E}-02 \\
1.620 \mathrm{E}-02 \\
1.227 \mathrm{E}-02 \\
8.260 \mathrm{E}-03 \\
7.100 \mathrm{E}-03 \\
5.255 \mathrm{E}-03 \\
4.171 \mathrm{E}-03 \\
2.654 \mathrm{E}-03 \\
\end{array}$ & $\begin{array}{l}3.701 \mathrm{E}-04 \\
2.515 \mathrm{E}-04 \\
1.905 \mathrm{E}-04 \\
1.283 \mathrm{E}-04 \\
1.103 \mathrm{E}-04 \\
8.161 \mathrm{E}-05 \\
6.478 \mathrm{E}-05 \\
4.115 \mathrm{E}-05 \\
\end{array}$ & $\begin{array}{l}2.024 \mathrm{E}-04 \\
1.376 \mathrm{E}-04 \\
1.042 \mathrm{E}-04 \\
7.016 \mathrm{E}-05 \\
6.031 \mathrm{E}-05 \\
4.464 \mathrm{E}-05 \\
3.543 \mathrm{E}-05 \\
2.252 \mathrm{E}-05 \\
\end{array}$ & $\begin{array}{c}0.000 \mathrm{E}+00 \\
2.142 \mathrm{E}-02 \\
3.244 \mathrm{E}-02 \\
4.369 \mathrm{E}-02 \\
4.694 \mathrm{E}-02 \\
5.212 \mathrm{E}-02 \\
5.516 \mathrm{E}-02 \\
5.941 \mathrm{E}-02 \\
\end{array}$ & $\begin{array}{l}4.881 \mathrm{E}-02 \\
4.388 \mathrm{E}-02 \\
4.135 \mathrm{E}-02 \\
3.876 \mathrm{E}-02 \\
3.801 \mathrm{E}-02 \\
3.682 \mathrm{E}-02 \\
3.612 \mathrm{E}-02 \\
3.514 \mathrm{E}-02 \\
\end{array}$ & $\begin{array}{r}-14.8065 \\
-12.8065 \\
-10.8065 \\
-6.8065 \\
-4.8065 \\
0.1935 \\
5.1935 \\
19.0500 \\
\end{array}$ & $\begin{array}{l}0.1114 \\
0.1639 \\
0.2164 \\
0.3214 \\
0.3738 \\
0.5051 \\
0.6363 \\
1.0000 \\
\end{array}$ & $\begin{array}{r}0.00 \\
1.29 \\
2.58 \\
5.16 \\
6.46 \\
9.68 \\
12.91 \\
21.86 \\
\end{array}$ \\
\hline m U235 & 2000 & $\mathrm{Vol} \mathrm{UO}_{2}$ & 215.020 & LenUO & 1.6974 & & & & & & \\
\hline WaterDepth & $L+W$ & $\mathrm{VFUO}_{2}$ & $\mathrm{VFH}_{2} \mathrm{O}$ & N235 & N234 & N238 & $\mathrm{NH}$ & NO & KENO-Z & Fract. full & $\mathrm{H} / \mathrm{U}$ mix \\
\hline $\begin{array}{l}0 \\
2\end{array}$ & 1.6974 & 1.0000 & 0.0000 & 2.383E-02 & 3.701E-04 & $2.024 \mathrm{E}-04$ & $0.000 E+00$ & $4.881 \mathrm{E}-02$ & -17.3526 & 0.0446 & 0.00 \\
\hline $\begin{array}{l}2 \\
4\end{array}$ & $\begin{array}{l}3.6974 \\
5.6974\end{array}$ & $\begin{array}{l}0.4591 \\
0.2979\end{array}$ & $\begin{array}{l}0.5409 \\
0.7021\end{array}$ & $\begin{array}{l}1.094 \mathrm{E}-02 \\
7.100 \mathrm{E}-03\end{array}$ & $\begin{array}{l}1.699 \mathrm{E}-04 \\
1.103 \mathrm{E}-04\end{array}$ & $\begin{array}{l}9.293 \mathrm{E}-05 \\
6.031 \mathrm{E}-05\end{array}$ & $\begin{array}{l}3.617 \mathrm{E}-02 \\
4.694 \mathrm{E}-02\end{array}$ & $\begin{array}{l}4.049 \mathrm{E}-02 \\
3.801 \mathrm{E}-02\end{array}$ & $\begin{array}{l}-15.3526 \\
-13.3526\end{array}$ & $\begin{array}{l}0.0970 \\
0.1495\end{array}$ & $\begin{array}{l}3.23 \\
6.46\end{array}$ \\
\hline 8 & 9.6974 & 0.1750 & 0.8250 & 4.171E-03 & $6.478 \mathrm{E}-05$ & $3.543 E-05$ & $5.516 \mathrm{E}-02$ & 3.612E-02 & -9.3526 & 0.2545 & 12.91 \\
\hline 10 & 11.6974 & 0.1451 & 0.8549 & $3.458 \mathrm{E}-03$ & 5.371E-05 & $2.937 \mathrm{E}-05$ & $5.716 \mathrm{E}-02$ & $3.566 \mathrm{E}-02$ & -7.3526 & 0.3070 & 16.14 \\
\hline $\begin{array}{l}15 \\
20\end{array}$ & 16.6974 & 0.1017 & 0.8983 & $2.423 \mathrm{E}-03$ & $3.762 \mathrm{E}-05$ & $2.058 \mathrm{E}-05$ & $6.006 \mathrm{E}-02$ & $3.499 \mathrm{E}-02$ & -2.3526 & 0.4383 & 24.21 \\
\hline $\begin{array}{r}20 \\
36.4027\end{array}$ & 21.6974 & 0.0782 & $\begin{array}{l}0.9218 \\
0.9555\end{array}$ & $1.864 \mathrm{E}-03$ & $2.895 \mathrm{E}-05$ & 1.584E-05 & $6.163 \mathrm{E}-02$ & $3.463 \mathrm{E}-02$ & 2.6474 & 0.5695 & 32.28 \\
\hline 36.4027 & 38.1000 & 0.0445 & 0.9555 & $1.062 \mathrm{E}-03$ & $1.646 \mathrm{E}-05$ & $9.007 \mathrm{E}-06$ & $6.388 \mathrm{E}-02$ & $3.412 \mathrm{E}-02$ & 19.0500 & 1.0000 & 58.76 \\
\hline
\end{tabular}

a Water is added to the top of the $\mathrm{UO}_{2}$ to the indicated depth and then mixed with the $\mathrm{UO}_{2}$. 
Table D7. Computer Input Spreadsheet Data for $\mathrm{UO}_{2}$ Dispersed into Receptacle

\section{$8 \mathrm{~kg}^{235} \mathrm{U}, \mathrm{H} / \mathrm{U}=10$ before dispersal}

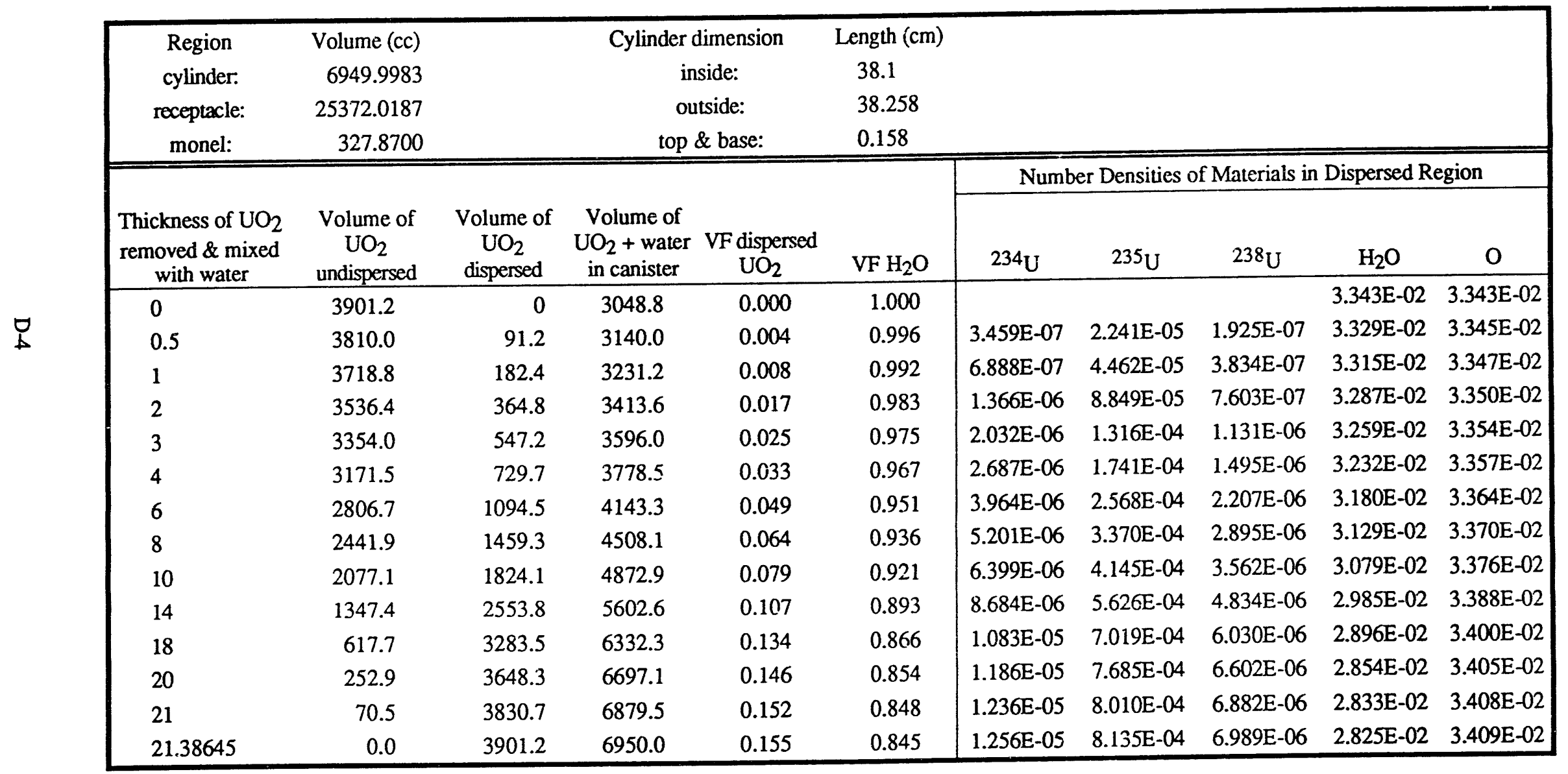


Table D8. Computer Input Spreadsheet Data for UNH Dispersed into Receptacle

$5564.9 \mathrm{~kg} 235 \mathrm{U}, \mathrm{UO}_{2}\left(\mathrm{NO}_{3}\right)_{2} \cdot 6\left(\mathrm{H}_{2} \mathrm{O}\right)$

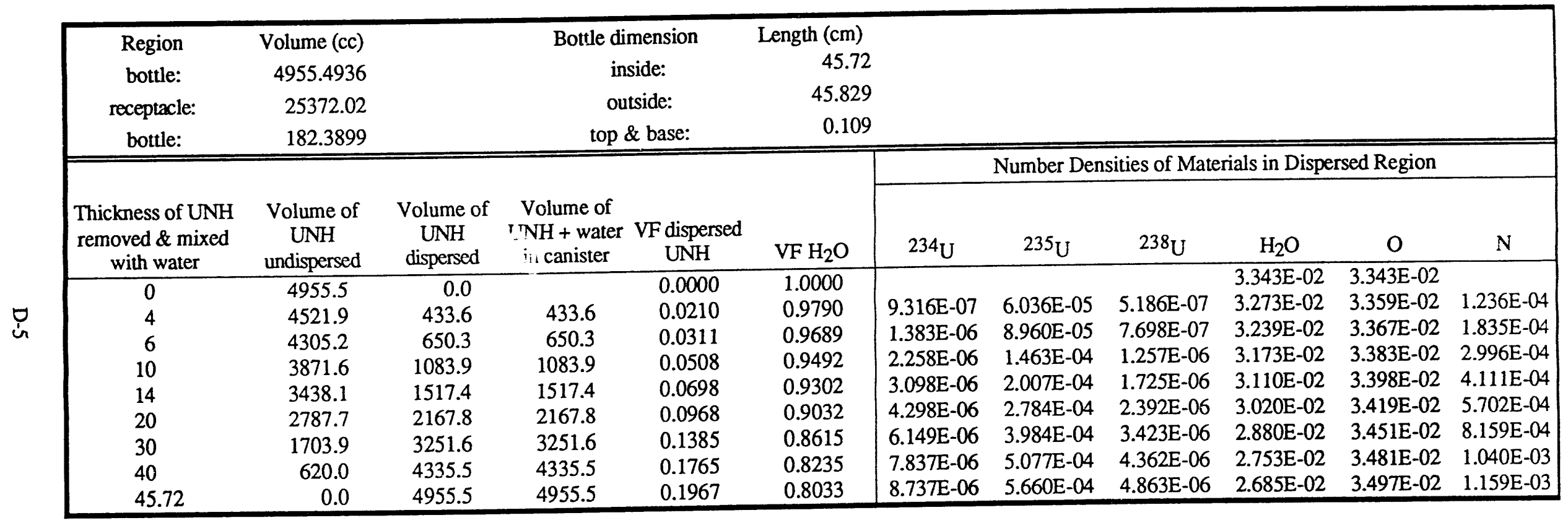


Table D9. Computer Input Spreadsheet Data for 8-inch UF 6 Cylinders with Admission of Water

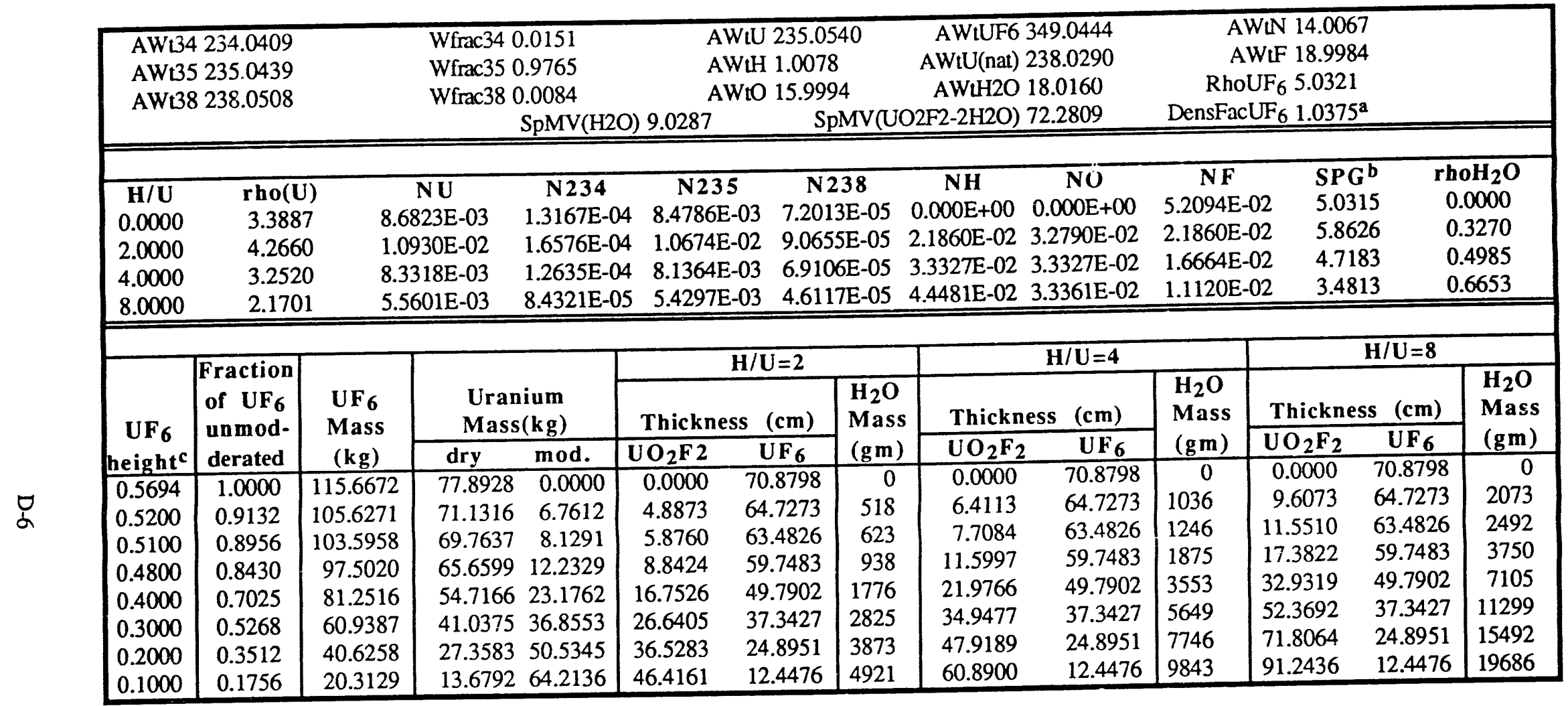

aDensity Factor $=(5.075)\left(\mathrm{MU} / \mathrm{MU}_{\text {nat }} / 4.85\right.$

where 5.075 is the density for unenriched $\mathrm{UF}_{6}$, from Ref. 9 ,

4.85 is the KENO default density,

$\mathrm{MU}$ is the molecular weight for enriched $\mathrm{UF}_{6}$,

and $\mathrm{MU}_{\text {nat }}$ is the molecular weight for unenriched $\mathrm{UF}_{6}$.

bSPG=(1/Na) $\sum \mathrm{N}_{\mathrm{i}} \mathrm{A}_{\mathrm{i}}$

where $\mathrm{N}_{\mathrm{a}}=$ Avogadro's number,

$\mathrm{N}_{\mathrm{i}}=$ Number density for material $\mathrm{i}$,

and $\mathrm{A}_{\mathrm{i}}=$ Atomic weight for material $\mathrm{i}$.

${ }^{c}$ (height of $U_{F}$ )/(cylinder height) 


\begin{tabular}{|c|c|c|c|c|c|}
\hline \multicolumn{6}{|c|}{ Appendix $\mathrm{E}$} \\
\hline Problem ID & keff & sigma & UF6_UO2F2_2_2825 & 0.9494 & 0.0030 \\
\hline \multicolumn{3}{|c|}{ UNH } & UF6_UO2F2_2_4921 & 1.0287 & 0.0033 \\
\hline UNH_12_5.6_M_M_0 & 0.7365 & 0.0049 & \multicolumn{3}{|c|}{$U_{F_{6}}(H / U=4)$, dry recept. } \\
\hline UNH_12_5.6_M_M_4 & 0.9104 & 0.0047 & UF6_UO2F2_4_1036 & 0.7781 & 0.0027 \\
\hline UNH_12_5.6_M_M_6 & 0.9592 & 0.0044 & UF6_UO2F2_4_1246 & 0.7896 & 0.0029 \\
\hline UNH_12_5.6_M_M_10 & 1.0352 & 0.0048 & UF6_UO2F2_4_3553 & 0.9391 & 0.0032 \\
\hline UNH_12_5.6_M_M_20 & 1.1268 & 0.0058 & UF6_UO2F2_4_5649 & 1.0094 & 0.0032 \\
\hline UNH_12_5.6_M_M_30 & 1.1781 & 0.0053 & UF6_UO2F2_4_7746 & 1.0445 & 0.0032 \\
\hline UNH_12_5.6_M_M_40 & 1.2024 & 0.0054 & UF6_UO2F2_4_9843 & 1.0736 & 0.0031 \\
\hline UNH_12_5.6_M_M_45.72 & 1.2216 & 0.006 & \multicolumn{3}{|c|}{$U_{6}(H / U=8)$, dry recept. } \\
\hline UNH_12_5.6_D_D_INF & 0.5823 & 0.0047 & UF6_UO2F2_8_2073 & 0.8081 & 0.0028 \\
\hline UNH_12_5.6_W_D_INF & 0.7239 & 0.0044 & UF6_UO2F2_8_2492 & 0.8342 & 0.0031 \\
\hline UNH_12_5.6_M_M_INF & 1.1132 & 0.0048 & UF6_UO2F2_8_3750 & 0.9170 & 0.0034 \\
\hline \multicolumn{3}{|c|}{$U F_{6} \quad(H / U=2)$} & UF6_UO2F2_8_7105 & 1.0097 & 0.0035 \\
\hline UF6_UO2F2_2_0 & 0.8288 & 0.0029 & UF6_UO2F2_8_11299 & 1.0688 & 0.0039 \\
\hline UF6_UO2F2_2_518 & 0.8313 & 0.0031 & UF6_UO2F2_8_15492 & 1.0907 & 0.0039 \\
\hline UF6_UO2F2_2_623 & 0.8297 & 0.0030 & UF6_UO2F2_8_19686 & 1.1171 & 0.0032 \\
\hline UF6_UO2F2_2_938 & 0.8499 & 0.0026 & \multicolumn{3}{|c|}{ Oxides in 6-inch Canisters } \\
\hline UF6_UO2F2_2_1776 & 0.9286 & 0.0031 & U308_15_5_W_D & 0.8775 & 0.0057 \\
\hline UF6_UO2F2_2_2825 & 1.0068 & 0.0033 & UO2_10_5_W_D & 0.7966 & 0.0045 \\
\hline UF6_UO2F2_2_3873 & 1.0519 & 0.0032 & UO2_10_10.5_W_D & 0.9755 & 0.0051 \\
\hline UF6_UO2F2_2_4921 & 1.0744 & 0.0032 & UO2_10_8_D_D & 0.8341 & 0.0059 \\
\hline \multicolumn{3}{|c|}{$U F_{6} \quad(H / U=4)$} & UO2_10_8_D_D_REG & 0.8267 & 0.0047 \\
\hline UF6_UO2F2_4_1036 & 0.8359 & 0.0029 & UO2_10_8_D_D_INF & 0.8448 & 0.0049 \\
\hline UF6_UO2F2_4_1246 & 0.8386 & 0.0030 & UO2_10_8_M_M_0.5 & 0.9423 & 0.0044 \\
\hline UF6_UO2F2_4_1875 & 0.8849 & 0.0031 & UO2_10_8_M_M_1 & 0.9690 & 0.0046 \\
\hline UF6_UO2F2_4_3553 & 0.9843 & 0.0035 & UO2_10_8_M_M_2 & 1.0104 & 0.0040 \\
\hline UF6_UO2F2_4_5649 & 1.0515 & 0.0036 & UO2_10_8_M_M_4 & 1.0864 & 0.0049 \\
\hline UF6_UO2F2_4_7746 & 1.0841 & 0.0034 & UO2_10_8_M_M_6 & 1.1578 & 0.0048 \\
\hline UF6_UO2F2_4_9843 & 1.1061 & 0.0037 & UO2_10_8_M_M_8 & 1.1826 & 0.0049 \\
\hline \multicolumn{3}{|c|}{$U F_{6} \quad(H / U=8)$} & UO2_10_8_M_M_10 & 1.2167 & 0.0057 \\
\hline UF6_UO2F2_8_2073 & 0.8592 & 0.0033 & UO2_10_8_M_M_14 & 1.2366 & 0.0052 \\
\hline UF6_UO2F2_8_2492 & 0.8904 & 0.0030 & UO2_10_8_M_M_18 & 1.2653 & 0.0049 \\
\hline UF6_UO2F2_8_3750 & 0.9535 & 0.0034 & UO2_10_8_W_D & 0.9146 & 0.0051 \\
\hline UF6_UO2F2_8_7105 & 1.0455 & 0.0036 & UO2_10_8_W_D_H2O & 0.8970 & 0.0046 \\
\hline UF6_UO2F2_8_11299 & 1.1015 & 0.0035 & UO2_10_8_W_D_INF & 0.9096 & 0.0051 \\
\hline UF6_UO2F2_8_15492 & 1.1164 & 0.0035 & UO2_10_8_W_M_8.6 & 0.9766 & 0.0055 \\
\hline UF6_UO2F2_8_19686 & 1.1220 & 0.0035 & UO2_10_8_W_M_16.7 & 1.0056 & 0.0049 \\
\hline \multirow{2}{*}{\multicolumn{3}{|c|}{$U F_{6}(H / U=2)$, dry recept. }} & UO2_10_8_W_W & 0.9303 & 0.0047 \\
\hline UF6_UO2F2_2_0 & 0.7611 & & UO2_10_8_W_W_INF & 0.9323 & 0.0054 \\
\hline UF6_UO2F2_2_518 & 0.7714 & 0.0028 & UO2_10_FULL_D_D & 0.9341 & 0.0053 \\
\hline UF6_UO2F2_2_623 & 0.7727 & 0.0028 & UO2_10_FULL_D_D_INF & 0.9472 & 0.0050 \\
\hline UF6_UO2F2_2_938 & 0.7918 & 0.0029 & UO2_10_FULL_W_D & 1.0147 & 0.0051 \\
\hline UF6_UO2F2_2_1776 & 0.8830 & 0.0031 & UO2_15_5_D_D & 0.8038 & 0.0053 \\
\hline & & & UO2_15_5_D_D_INF & 0.8265 & 0.0051 \\
\hline
\end{tabular}




\begin{tabular}{|c|c|c|c|c|c|}
\hline \multirow{3}{*}{$\begin{array}{l}\text { UO2_15_5_W_D } \\
\text { UO2_15_5_W_D_H2O } \\
\text { UO2_15_5_W_D_INF }\end{array}$} & \multirow{3}{*}{$\begin{array}{l}0.8842 \\
0.8818 \\
0.8962\end{array}$} & \multirow{3}{*}{$\begin{array}{l}0.0049 \\
0.0045 \\
0.0053\end{array}$} & \multirow{2}{*}{\multicolumn{3}{|c|}{$\begin{array}{c}\text { Oxides in 6-Inch Canisters, } 8 \mathrm{~kg} 235 \mathrm{U} \text {, } \\
\text { concrete } H \text { variation }\end{array}$}} \\
\hline & & & & & \\
\hline & & & UO2_10_8_D_D_INF_OH: & 0.9204 & 0.0048 \\
\hline UO2_15_5_W_M_5 & 0.9415 & 0.0052 & UO2_10_8_D_D_INF_.2H & 0.8690 & 0.0049 \\
\hline UO2_15_5_W_M_15 & 1.0002 & 0.0065 & UO2_10_8_D_D_INF_.5H & 0.8509 & 0.0051 \\
\hline UO2_15_5_W_M_20 & 1.0106 & 0.0049 & UO2_10_8_D_D_INF & 0.8448 & 0.0049 \\
\hline UO2_15_5_W_W_INF & 0.9121 & 0.0053 & UO2_10_8_D_D_INF_2H: & 0.8354 & 0.0053 \\
\hline UO2_15_8_W_D & 0.9977 & 0.0051 & \multicolumn{3}{|c|}{ Oxides in 6-Inch Canisters, $8 \mathrm{~kg} 235 \mathrm{U}$, } \\
\hline UO2_15_FULL_D_D & 0.9575 & 0.0056 & $\begin{array}{l}\mathrm{UO}_{2} \text { dens! } \\
\text { UO2 } \mathrm{C} \& \mathrm{D} \mathrm{D} \text { INF } 0\end{array}$ & & 0,0039 \\
\hline UO2_15_FULL_D_D_INF & 0.9656 & 0.0060 & UO2_C_8_D_D_INF_2 & 0.4614 & 0.0036 \\
\hline UO2_15_FULL_W_D & 1.0280 & 0.0050 & UO2_C_8_D_D_INF_4 & 0.4270 & 0.0033 \\
\hline UO2_65_1.2_W_D & 0.8385 & 0.0055 & UO2_C_8_D_D_INF_8 & 0.4017 & 0.0029 \\
\hline UO2_65_2_D_D & 0.8600 & 0.0058 & UO2_C_8_D_D_INF_10 & 0.3909 & 0.0038 \\
\hline UO2_65_2_D_D_H2O & 0.9353 & 0.0052 & UO2_C_8_D_D_INF_20 & 0.3835 & 0.0035 \\
\hline UO2_65_2_D_D_INF & 0.8712 & 0.0056 & UO2_C_8_D_D_INF_33 & 0.3819 & 0.0033 \\
\hline UO2_65_2_W_D & 0.9471 & 0.0053 & \multicolumn{3}{|c|}{ Oxides in 5-Inch Canisters } \\
\hline UO2_65_2_W_D_INF & 0.9623 & 0.0057 & U308_15_3.47_W_D & 0.7873 & 0.0055 \\
\hline UO2_65_2_W_M_7 & 0.9760 & 0.0053 & UO2__10_3.47_W_D & 0.7258 & 0.0052 \\
\hline UO2_65_2_W_M_8 & 0.9810 & 0.0050 & UO2_10_5.56_D_D & 0.7058 & 0.0048 \\
\hline UO2_65_2_W_M_10 & 0.9757 & 0.0048 & UO2_10_5.56_D_D_INF & 0.7063 & 0.0045 \\
\hline UO2_65_2_W_W_INF & 0.9662 & 0.0062 & UO2_10_5.56_W_D & 0.8345 & 0.0049 \\
\hline UO2_65_FULL_D_D & 0.9158 & 0.0060 & UO2_10_5.56_W_D_H2O & 0.8204 & 0.0051 \\
\hline UO2_65_FULL_D_D_INF & 0.9353 & 0.0051 & UO2_10_5.56_W_D_INF & 0.8364 & 0.0056 \\
\hline UO2_65_FULL_W_D & 0.9930 & 0.0056 & UO2_10_5.56_W_W & 0.8369 & 0.0044 \\
\hline UO3_15_5_D_D & 0.8138 & 0.0052 & UO2_10_5.56_W_W_INF & 0.8340 & 0.0046 \\
\hline UO3_15_5_W_D & 0.8902 & 0.0055 & UO2_10_7.29_W_D & 0.8752 & 0.0057 \\
\hline \multicolumn{3}{|c|}{ Oxides In 6-Inch Canisters, $8 \mathrm{~kg} 235 \mathrm{U}$} & UO2_10_8_W_D & 0.8964 & 0.0047 \\
\hline UO2_C_8_W_M_0 & 0.6497 & 0.0037 & UO2_10_8_W_M_5 & 0.9024 & 0.0051 \\
\hline UO2_C_8_W_M_4 & 0.7501 & 0.0043 & UO2_10_8_W_M_7 & 0.9068 & 0.0050 \\
\hline UO2_C_8_W_M_8 & 0.8345 & 0.0052 & UO2_10_FULL_D_D & 0.8145 & 0.0053 \\
\hline UO2_C_8_W_M_10 & 0.8463 & 0.0047 & UO2_10_FULL_D_D_INF & 0.8181 & 0.0049 \\
\hline UO2_C_8_W_M_15 & 0.9150 & 0.0051 & UO2_10_FULL_W_D & 0.9102 & 0.0054 \\
\hline UO2_C_8_W_M_17 & 0.9183 & 0.0052 & UO2_15_3.47_D_D & 0.6742 & 0.0047 \\
\hline UO2_C_8_W_M_20 & 0.9520 & 0.0057 & UO2_15_3.47_D_D_INF & 0.6809 & 0.0048 \\
\hline UO2_C_8_W_M_33 & 1.0016 & 0.0048 & UO2_15_3.47_W_D & 0.8026 & 0.0047 \\
\hline \multicolumn{3}{|c|}{ Oxides In 6-Inch Canisters, $5 \mathrm{~kg}{ }^{235} \mathrm{U}$} & UO2_15_3.47_W_D_H2O & 0.7959 & 0.0049 \\
\hline UO2_C_5_W_M_O & 0.4813 & 0.0036 & UO2_15_3.47_W_D_INF & 0.8071 & 0.0054 \\
\hline UO2_C_5_W_M_2 & 0.6187 & 0.0037 & UO2_15_3.47_W_W_INF & 0.8277 & 0.0051 \\
\hline UO2_C_5_W_M_10 & 0.8166 & 0.0049 & UO2_15_5.56_W_D & 0.8872 & 0.0047 \\
\hline UO2_C_5_W_M_18.4 & 0.9103 & 0.0050 & UO2_15_5_W_D & 0.8718 & 0.0060 \\
\hline UO2_C_5_W_M_20 & 0.9309 & 0.0055 & UO2_15_5_W_M_5 & 0.8932 & 0.0053 \\
\hline UO2_C_5_W_M_35.2 & 0.9941 & 0.0060 & UO2_15_5_W_M_7 & 0.9017 & 0.0052 \\
\hline \multicolumn{3}{|c|}{ Oxides in 6-Inch Canisters, $2 \mathrm{~kg}{ }^{235} \mathrm{U}$} & UO2_15_5_W_M_10 & 0.9081 & 0.0051 \\
\hline UO2_C_2_W_M_O & 0.2957 & 0.0025 & UO2_15_5_W_M_12 & 0.9212 & 0.0052 \\
\hline UO2_C_2_W_M_2 & 0.5047 & 0.0037 & UO2_15_FULL_D_D & 0.8015 & 0.0052 \\
\hline UO2_C_2_W_M_8 & 0.7198 & 0.0044 & UO2_15_FULL_D_D_INF & 0.8254 & 0.0046 \\
\hline UO2_C_2_W_M_10 & 0.7670 & 0.0050 & UO2_15_FULL_W_D & 0.9309 & 0.0053 \\
\hline UO2_C_2_W_M_20 & 0.9137 & 0.0049 & UO2_65_1.39_D_D & 0.7196 & 0.0056 \\
\hline UO2_C_2_W_M_36.9 & 0.9618 & 0.0053 & UO2_65_1.39_D_D_H2O & 0.8308 & 0.0053 \\
\hline
\end{tabular}




\begin{tabular}{|c|c|c|}
\hline UO2_65_1.39_D_D_INF & 0.7118 & 0.0048 \\
\hline UO2_65_1.39_W_D & 0.8535 & 0.0052 \\
\hline UO2_65_1.39_W_D_INF & 0.8371 & 0.0053 \\
\hline UO2_65_1_W_D & 0.7856 & 0.0052 \\
\hline UO2_65_1_W_M_5 & 0.8730 & 0.0053 \\
\hline UO2_65__1_W_M_10 & 0.8294 & 0.0052 \\
\hline UO2_65_1_W_M_17.8 & 0.8389 & 0.0049 \\
\hline UO2_65_FULL_D_D_INF & 0.7705 & 0.0054 \\
\hline \multicolumn{3}{|c|}{$\begin{array}{c}\text { Oxides In 5-Inch Canisters, } \\
8 \text { kg U-235 }\end{array}$} \\
\hline UO2_C_8_W_M_O & 0.6661 & 0.0042 \\
\hline UO2_C_8_W_M_2 & 0.6973 & 0.0038 \\
\hline UO2_C_8_W_M_4 & 0.7332 & 0.0044 \\
\hline UO2_C_8_W_M_8 & 0.7904 & 0.0047 \\
\hline UO2_C_8_W_M_10 & 0.7959 & 0.0049 \\
\hline UO2_C_8_W_M_15 & 0.8364 & 0.0048 \\
\hline UO2_C_8_W_M_20 & 0.8704 & 0.0052 \\
\hline UO2_C_8_W_M_31.3 & 0.9035 & 0.0052 \\
\hline
\end{tabular}




\section{Explanation of Problem ID Coding:}

The above summary table includes a Problem ID. The coding we use helps provide an immediate overview of the entire problem, and makes it easy to find the corresponding KENO V.a problem input in Appendix F. The Problem ID is described in the example below:

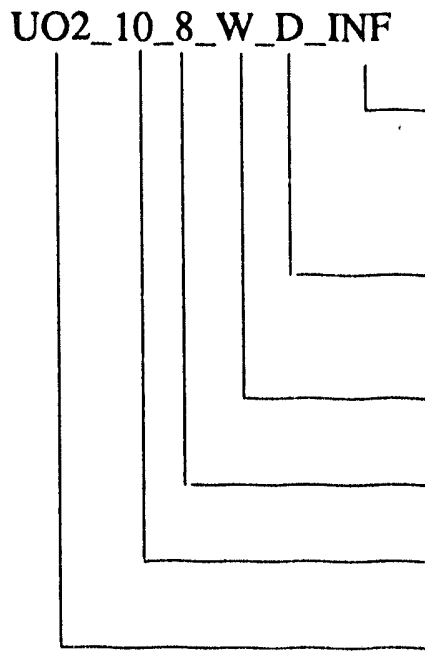

KENO Problem ID Coding Example

\section{External boundary}

(REG="REGULAR CONCRETE"; H2O=water-reflection; INF=infinite planar array; blank $=$ single unit)

Canister interior flooding

(W=flooded; $D=$ =unflooded; $M=$ mixture of water \& fissile material)

Receptacle flooding (W=flooded; $D=$ unfloocitd)

Mass of ${ }^{235} \mathrm{U}(\mathrm{kg})$

$\mathrm{H} / \mathrm{U}$

Form of fissile material

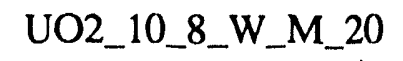

Depth of the water region above the $\mathrm{UO}_{2}$ prior to the mixing

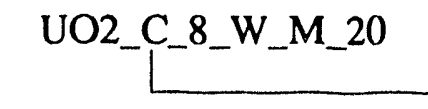

UNH_12_5.6_M_M_10

In this series the water is mixed with $\mathrm{UO}_{2}$ which is initially at crystal density

Thickness of the UNH region removed and mixed with water in the receptacle

UF6_UO2F2_2_4914

Volume of water (cc) admited to the cylinder and mixed with some of the $\mathrm{UF}_{6}$ to form $\mathrm{UO}_{2} \mathrm{~F}_{2} \cdot \mathrm{H}_{2} \mathrm{O}$. (sec Fig. 3)

This is the $\mathrm{H} / \mathrm{U}$ in the $\mathrm{UO}_{2} \mathrm{~F}_{2} \cdot \mathrm{H}_{2} \mathrm{O}$

UO2_10_8_D_D_INF_.2H

This is the density of hydrogen in the concrete relative to the normal density 


\section{Appendix $\mathbf{F}$ \\ KENO Problem Inputs}

UNH

\section{-CSAS25}

UNH_12_5.6_M_M_O

27GROOUPNDF4 TINFHOMMEDIUM

U-234 1 0.0 4.4411E-05

U-235 $10.02 .8773 E-03$

U-238 $10.02 .4721 E-05$

H $10.03 .5357 \mathrm{E}-02$

O 1 0.0 4.1250E-02

N 1 0.0 5.8928E-03

C 20.0 .04298

H 20.0 .08537

AL $30.05 .54-4$

CA $30.05 .30-3$

C $30.06 .59-3$

H 30.0 .0133

O 30.0 .0457

FE $30.03 .59-4$

MG $30.03 .25-3$

SI $30.08 .43-3$

NA $30.03 .89-4$

S $30.01 .09-4$

$\mathrm{H} 2 \mathrm{O} 41.0$

CARBONSTEEL 51.0

END COMP

UNH $125.6 \mathrm{MM} 0$

REA $\bar{D}$ PARAA $\bar{M} \overline{\mathrm{UU}} \overline{\mathrm{N}}=\mathrm{Y} E S$ END PARAM

READ GEOM

CYLIN!DER 115.8737526 .691 -19.029

CYLINDER $215.9737526 .7-19.129$

CYLINDER $4110.9537548 .181-19.129$

CYLINDER $5111.2458549 .451-19.764$

CUBOID $3350-5050-5049.451-50.549$

END GEOM

READ BNDS $X F C=V A C$ YFC $=V A C$ END BNDS

READ PLOT TTL=IUNH_12 5.6_M_M_OI

$P L T=Y E S$ PIC $=M I X T U R \bar{E} X \bar{U} L=-50 \bar{Y} \bar{L}=00$

$Z U L=50 \quad X L R=50$ YLR $=00 \quad Z L R=-30$

$U A X=1$ WDN $=-1 \quad \mathrm{NAX}=130 \mathrm{LPI}=8$

$\mathrm{NCH}=$ ! UFCW I END PLOT

END DATA

END

\section{$=$ CSAS25}

UNH_12 5.6 M_M 4

27GROUUPNDF4 INFHOMMEDIUM

U-234 10.0 4.4411E-05

U-235 $10.02 .8773 E-03$

U-238 $10.02 .4721 E-05$

H $10.03 .5357 E-02$

$010.04 .1250 \mathrm{E}-02$

$\mathrm{N} 10.05 .8928 \mathrm{E}-03$

C 20.0 .04298

H 20.0 .08537

AL $30.05 .54-4$

CA $30.05 .30-3$

C $30.06 .59-3$

H 30.0 .0133

O 30.0 .0457

FE $30.03 .59-4$

MG $30.03 .25-3$

SI $30.08 .43-3$

NA $30.03 .89-4$
S $30.01 .09-4$

U-234 $40.09 .3163 E-07$

U-235 $40.06 .03573 E-05$

U-238 $40.05 .18575 E-07$

H 40.00 .066199151

040.00 .033594036

N 40.00 .000123615

CARBONSTEEL 51.0

END COMP

UNH_12_5.6_M_M_4

READ GEOM

CYLINDER 115.8737522 .691 -19.029

CYLINDER $415.8737526 .691-19.029$

CYLINDER 215.97375 26.7 -19.129

CYLINDER $4110.9537548 .181-19.129$

CYLINDER $5111.2458549 .451-19.764$

CUBOID $3350-5050-5049.451-50.549$

END GEOM

READ BNDS XFC $=V A C$ YFC $=V A C$ END BNDS

READ PLOT TTL=IUNH 12 5.6 M M 4 4!

$P L T=Y E S$ PIC $=M I X T U R \bar{E} X \bar{U} L=-50$ YUL $=00$

$Z U L=50$ XLR $=50$ YLR $=00$ ZLR $=-30$

$U A X=1$ WDN $=1 \quad N A X=130 \quad L P \mid=8$

$\mathrm{NCH}=$ !UFCW IEND PLOT

END DATA

END

\section{$=$ CSAS 25}

UNH $125.6 \mathrm{M} \mathrm{M} 6$

27GR̄OUPNDF4 INFHOMMEDIUM

U-234 1 0.0 4.4411E-05

U-235 $10.02 .8773 E-03$

U-238 1 0.02 2.4721E-05

H $10.03 .5357 \mathrm{E}-02$

O $10.04 .1250 \mathrm{E}-02$

N 1 0.0 5.8928E-03

C 20.0 .04298

H 20.0 .08537

AL $30.05 .54-4$

CA $30.05 .30-3$

C $30.06 .59-3$

H3 0.0 .0133

O 30.0 .0457

FE 3 0.0 3.59-4

MG $30.03 .25-3$

SI $30.08 .43-3$

NA $30.03 .89-4$

S $30.01 .09-4$

U-234 $40.01 .3829 E-06$

U-235 40.0 8.9596E-05

U-238 $40.07 .6979 E-07$

H $40.06 .5879 E-02$

O 40.0 3.3673E-02

N 40.01 .8350 E-04

CARBONSTEEL 51.0

END

END

END

END

END

END

END

END

END

END

END

END

END

END

END

END

END

END

END

END

END

END

END

END

END COMP

UNH_12 5.6_M_M_6

READ GEOM

CYLINDER $115.8737520 .691-19.029$

CYLINDER 415.8737526 .691 -19.029

CYLINDER $215.9737526 .7-19.129$

CYLINDER $4110.9537548 .181-19.129$

CYLINDER $5111.2458549 .451-19.764$

CUBOID $3350-5050-5049.451-50.549$ 
END GEOM

READ BNDS XFC VAC YFC $=V A C$ END ENDS

READ PLOT TTL=!UNH_12_5.6_M_M_6!

PLT=YES PIC-MIXTURE XÜL $=-50$ YŨL $=0$

ZUL-50 XLR-50 VLR=00 ZLR=-30

$U A X=1$ WDN $=1$ NAX $=130 \mathrm{LPI}=8$

NCH=IUFCWIEND PLOT

END DATA

END

-CSAS25

UNH 125.6 M M 10

27GR̄OUPNDF4 INFFHOMMEDIUM

U-234 10.0 4.4411E-05

U-235 $10.02 .8773 E-03$

U-238 $10.02 .4721 E-05$

H $10.03 .5357 E-02$

O $10.04 .1250 E-02$

N $10.05 .8928 E-03$

C 20.0 .04298

H 20.0 .08537

AL $30.05 .54-4$

CA $30.05 .30-3$

C $30.06 .59-3$

H 30.0 .6133

O 30.0 .0457

FE $30.03 .59-4$

MG $30.03 .25-3$

SI $30.08 .43-3$

NA 30.03 .89 .4

$S 30.01 .09-4$

U.234 $40.02 .2580 E-06$

U-235 $40.01 .4629 E-04$

U-238 40.0 1.2569E-06

H $40.06 .5258 E-02$

O $40.03 .3828 E-02$

N $40.02 .9961 E-04$

CARBONSTEEL 51.0

END

END

END

END

END

END

END

END

END

END

END

ENC

END

END

END

END

END

END

END

END

END

END

END

END

END

UNH_12_5.6_M_M_10

READ GEOM

CYINDER $115.8737516 .691-19.029$

CYI:HOER $415.8737526 .691-19.029$

CYINDER 215.9737526 .7 -19.129

CVIINDER $4110.9537548 .181-19.129$

CYINDER $5111.2458549 .451-19.764$

CUBOID $3350-5050-50 \quad 49.451-50.549$

END GEOM

READ BNDS XFC-VAC YFC=VAC END BNDS

READ PLOT TTL=IUNH_12_5.6_M_M_10!

PLT $=Y E S$ PIC=MIXTURE XUL $=-50$ YUL $=00$

$Z U L=50$ XLR $=50$ YLR $=00$ ZLR $=-30$

$U A X=1$ WDN $=-1 \quad N A X=130 \quad \angle P I=8$

NCH $=$ !UFCWIEND PLOT

END DATA

END

\section{-CSAS25}

UNH 125.6 M M 20

27GROUPNDF4 INFHOMMEDIUM

U-234 $10.04 .4411 E-05$

U.235 $10.02 .8773 E-03$

U-238 $10.02 .4721 E-05$

END

H 1 0.0 3.5357E-02
O $10.04 .1250 E-02$

END

N $10.05 .8928 E-03$

END

C 2 0.0 .04298

H 20.0 .08537

AL $30.05 .54-4$

CA $30.05 .30-3$

C $30.06 .59-3$

H 30.0 .0133

O 30.0 .0457

FE $30.03 .59-4$

MG $30.03 .25-3$

SI $30.08 .43-3$

NA $30.03 .89-4$

S $30.01 .09-4$

U-234 $40.04 .2975 E-06$

U.235 $40.02 .7842 E-04$

U-238 $40.02 .3921 E-06$

H $40.06 .3812 E-02$

O $40.03 .4187 E-02$

N $40.05 .7023 E-04$

CARBONSTEEL 51.0

END COMP

UNH_12 5.6_M_M_2O

READ̄ GEOM

CYLINDER $115.873756 .691-19.029$

CYLINDER $415.8737526 .691-19.029$

CYIINDER $215.9737526 .7-19.129$

CYLINDER $4110.9537548 .181-19.129$

CYLINDER $5111.2458549 .451-19.764$

CUBOID $3350-5050-5049.451 \quad-50.549$

\section{END GEOM}

READ BNDS XFC-VAC YFC-VAC END BNDS

READ PLOT TTL=!UNH_12_5.6_M_M_20!

PLT $=$ YES PIC $=M I X T U R \bar{E}$ XŪL $=-50 \bar{Y} \bar{L}=00$

$Z U L=50$ XLR $=50$ YLR $=00$ ZLR $=-30$

$U A X=1$ WDN $=-1 \quad N A X=130 \quad L P I=8$

$\mathrm{NCH}=$ !UFCWIEND PLOT

END DATA

END

$=$ CSAS25

UNH 12 5.6_M_M_30

27GROOUPNDF4 INFHOMMEDIUM

U-234 $10.04 .4411 E-05$

$\mathrm{U}-23510.02 .8773 \mathrm{E}-03$

U-238 $10.02 .4721 E-05$

H $10.03 .5357 E-02$

$010.04 .1250 E-02$

N $10.05 .8928 E-03$

C 20.0 .04298

H 20.0 .08537

AL $30.05 .54-4$

CA $30.05 .30-3$

C $30.06 .59-3$

H 30.0 .0133

030.0 .0457

FE $30.03 .59-4$

MG $30.03 .25-3$

SI $30.08 .43-3$

NA $30.03 .89-4$

S $30.01 .09-4$

U-234 $40.06 .1488 E-06$

U-235 $40.03 .9836 E-04$

U-238 $40.03 .4226 E-06$

END

END

END

END

END

END

END

END

END

END

END

END

END

END

END

END

END

END

END

END

END 


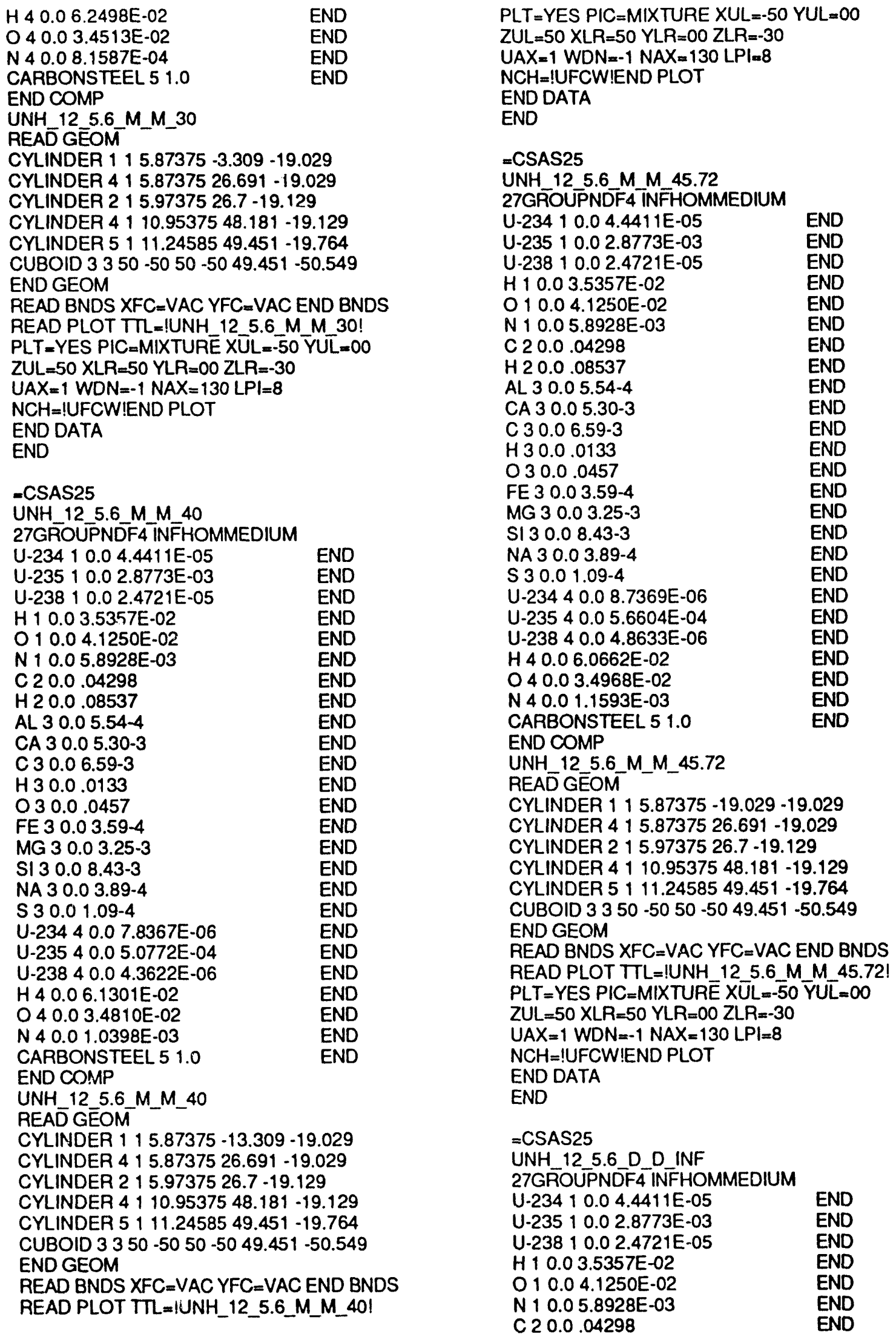




\begin{tabular}{|c|c|c|c|}
\hline 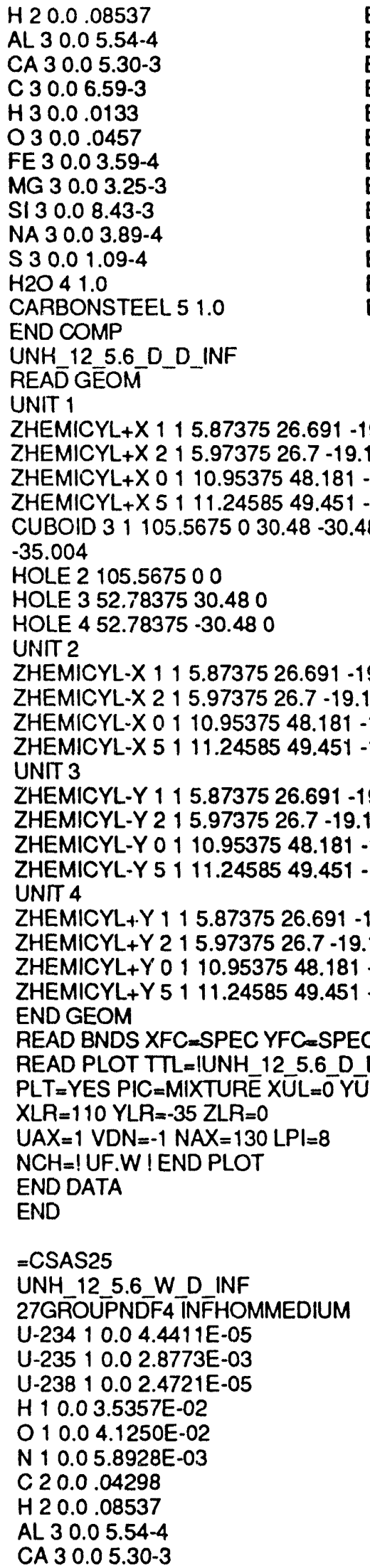 & $\begin{array}{l}\text { END } \\
\\
19.029 \\
129 \\
-19.129 \\
-19.764 \\
4849.451 \\
\\
\\
19.029 \\
129 \\
-19.129 \\
-19.764 \\
19.029 \\
129 \\
-19.129 \\
-19.764 \\
19.029 \\
129 \\
-19.129 \\
-19.764 \\
C E N D \text { BNDS } \\
D \text { INF! } \\
L=35 \text { ZUL }=0 \\
\end{array}$ & 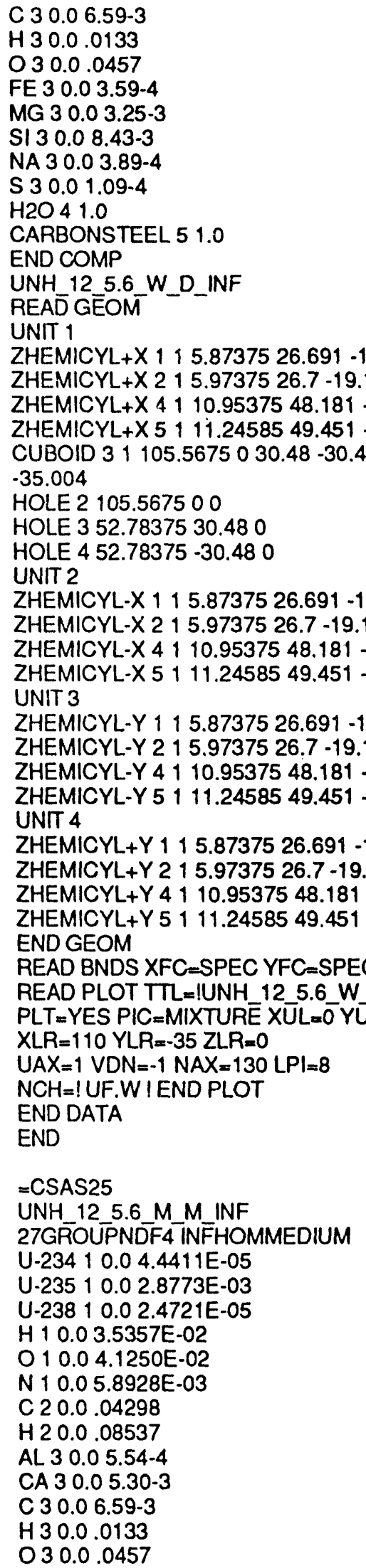 & $\begin{array}{l} \\
19.029 \\
129 \\
-19.129 \\
-19.764 \\
1849.451 \\
\\
\\
19.029 \\
129 \\
-19.129 \\
-19.764 \\
19.029 \\
129 \\
-19.129 \\
-19.764 \\
19.029 \\
1129 \\
-19.129 \\
-19.764 \\
C E N D \text { BNDS } \\
D \text { INFI } \\
\text { L } 35 \text { ZUL }=0 \\
\end{array}$ \\
\hline
\end{tabular}


FE $30.03 .59-4$

MG $30.03 .25-3$

SI $30.08 .43-3$

NA $30.03 .89-4$

S $30.01 .09-4$

$\mathrm{H} 2 \mathrm{O} 41.0$

CARBONSTEEL 51.0

END COMP

UNH 12 5.6 M_M_INF

READ GEOM

UNTT 1

ZHEMICYL+X $1110.9537548 .181-19.129$

ZHEMICYL+X $5111.2458549 .451-19.764$

CUBOID $31105.5675030 .48-30.4849 .451$

$-35.004$

HOLE 2105.567500

HOLE 352.7837530 .480

HOLE $452.78375-30.480$

UNIT 2

ZHEMICYL-X 1 1 10.95375 48.181 -19.129

ZHEMICYL-X $5111.2458549 .451-19.764$

UNIT 3

ZHEMICYL-Y $1110.9537548 .181-19.129$

ZHEMICYL-Y $5111.2458549 .451-19.764$

UNIT 4

ZHEMICYL+Y $1110.9537548 .181-19.129$

ZHEMICYL+Y $5111.2458549 .451-19.764$

END GEOM

READ BNDS XFC $=S P E C$ YFC $=S P E C$ END BNDS

READ PLOT TTL $=$ !UNH $125.6 \mathrm{M} \mathrm{M}$ INF!

PLT $=Y E S$ PIC $=M I X T U R \bar{E} X \bar{U} L=\overline{0} Y \bar{U} L=35$ ZUL $=0$

$X L R=110 Y L R=-35$ ZLR $=0$

$U A X=1$ VDN $=-1 \quad N A X=130 \mathrm{LPl}=8$

$N C H=$ ! UF.W ! END PLOT

END DATA

END

$$
\text { UF6 }(H / U=2)
$$

\section{-CSAS25}

UF6 UO2F2 2 -

27GR $\bar{R} O U P N \bar{F} \overline{4}$ INFHOMMEDIUM

UF6 11.03752939223597 .65922380 .8592234 1.5000

CARBONSTEEL 21.0

AL $30.05 .54-4$

CA $30.05 .30-3$

C $30.06 .59-3$

H 30.0 .0133

O 30.0 .0457

FE $30.03 .59-4$

MG $30.03 .25-3$

SI $30.08 .43-3$

NA $30.03 .89-4$

S $30.01 .09-4$

$\mathrm{H} 2 \mathrm{O} 41.0$

NI 50.0 .0603

CU 50.0 .02649

FE 50.0 .00119

MN 50.0 .0006

SOLNUO2F2 642660 SPG $=5.86331 .0000293$

9223597.65922380 .85922341 .5000

END COMP

UF6 UO2F2 20

REA $\bar{D}$ PARAM $\overline{\mathrm{G}}=\mathrm{N}=203$ END PARAM
READ GEOM

CYLINDER $1110.16-78.5130-149.3928$

CYLINDER $0110.16-24.9172-149.3928$

CYLINDER $5110.6273-24.45$-149.86

CYLINDER $4117.22440-149.86$

CYLINDER $2117.781 .27-150.495$

CUBOID $3250-5050-501.27-180$

END GEOM

READ BNDS XFC $=V A C$ YFC $=V A C$ END BNDS

READ PLOT TTL=IUF6_UO2F2_2_01

$P L T=N O P I C=M I X T U R E \bar{E} X L=-50$ YUL $=00 \quad Z U L=5$

$X L R=50$ YLR $=00 Z L R=-185$

$U A X=1$ WDN $=-1 \quad N A X=130 \quad L P I=8$

$\mathrm{NCH}=$ ! UFCWMSI END PLOT

END DATA

END

-CSAS25

UF6 UO2F2 2518

27GRिOUPNDF $\overline{4}$ INFHOMMEDIUM

UF6 11.03752939223597 .65922380 .8592234

1.5000

CARBONSTEEL.2 1.0 END

AL $30.05 .54-4$

CA $30.05 .30-3$

C $30.06 .59-3$

H 30.0 .0133

O 30.0 .0457

FE $30.03 .59-4$

MG $30.03 .25-3$

SI $30.08 .43-3$

NA $30.03 .89-4$

S $30.01 .09-4$

$\mathrm{H} 2 \mathrm{O} \quad 41.0$

NI 50.0 .0603

CU 50.0 .02649

FE 50.0 .00119

MN 50.0 .0006

END

END

END

END

END

END

END

END

END

END

END

END

END

SOLNUO2F2 642660 SPG $=5.86331 .0000293$ 9223597.65922380 .85922341 .5000 END COMP

UF6 UO2F2 2 518

RE $\triangle \bar{D}$ PARAM $\bar{G} \overline{\mathrm{G}}=203$ END PARAM

READ GEOM

CYLINDER 1110.16 -84.6655 -149.3928

CYLINDER $6110.16 \quad-79.7782-149.3928$

CYLINDER $0110.16-24.9172-149.3928$

CYLINDER $5110.6273-24.45-149.86$

CYLINDER $4117.22440-149.86$

CYLINDER $2117.781 .27-150.495$

CUBOID $3250-5050-501.27-180$

END GEOM

READ BNDS XFC $=V A C$ YFC $=V A C$ END BNDS

READ PLOT TTL=IUF6 UO2F2 2 518 !

PLT $=$ NO PIC $=M I X T U R E \bar{E}$ XUL $=-50$ YUL $=00$ ZUL $=5$

$X L R=50$ YLR $=00$ ZLR $=-185$

$U A X=1$ WDN $=-1 N A X=130$ LPI $=8$

$\mathrm{NCH}=$ ! UFCWMS! END PLOT

END DATA

END

$=$ CSAS25

UF6 UO2F2 2623

27GR̄OUPNDF $\overline{4}$ INFHOMMEDIUM 
UF6 $1 \quad 1.03752939223597 .65922380 .8592234$ 1.5000

CARBONSTEEL 21.0

AL $30.05 .54-4$

CA $30.05 .30-3$

C $30.06 .59-3$

H 30.0 .0133

O $\quad 30.0 .0457$

FE $30.03 .59-4$

MG $30.03 .25-3$

SI $30.08 .43-3$

NA $30.03 .89-4$

S $30.01 .09-4$

$\mathrm{H} 2 \mathrm{O} 41.0$

NI 50.0 .0603

CU 50.0 .02649

FE 50.0 .00119

MN 50.0 .0006

END

END

END

END

END

END

END

END

END

END

END

END

END

END

END

END

END

SOLNUO2F2 642660 SPG $=5.86331 .0000293$

9223597.65922380 .85922341 .5000

END COMP

UF6 UO2F2 2623

READ PARAM $\bar{G} E N=203$ END PARAM

READ GEOM

CYLINDER $1110.16-85.9102-149.3928$

CYLINDER $6110.16 \quad-80.0342-149.3928$

CYLINDER $0110.16-24.9172-149.3928$

CYLINDER $5110.6273-24.45$-149.86

CYLINDER $4117.22440 \cdot 149.86$

CYLINDER $2117.781 .27-150.495$

CUBOID $3250-5050-501.27-180$

END GEOM

READ BNDS XFC=VAC YFC $=V A C$ END BNDS

READ PLOT TTL=IUF6 UO2F2 2 623!

$P L T=N O P I C=M I X T U R E X U L=-50 \bar{Y} U L=00 Z U L=5$

$X L R=50$ YLR $=00$ ZLR $=-185$

$U A X=1$ WDN $=-1$ NAX $=130 \quad L P I=8$

$\mathrm{NCH}=$ I UFCWMS! END PLOT

END DATA

END

=CSAS25

UF6 UO2F2 2938

27GR̄OUPNDF $\overline{4}$ INFHOMMEDIUM

UF6 11.03752939223597 .65922380 .8592234

1.5000

AL $30.05 .54-4$

CA $30.05 .30-3$

C $30.06 .59-3$

H 30.0 .0133

O 30.0 .0457

FE $30.03 .59-4$

MG $30.03 .25-3$

SI $30.08 .43-3$

NA $30.03 .89-4$

S 30.01 .094

$\mathrm{H} 2 \mathrm{O} 41.0$

NI 50.0 .0603

CU 50.0 .02649

FE 50.0 .00119

MN 50.0 .0006

SOLNUO2F2 642660 SPG $=5.86331 .0000293$

9223597.65922380 .85922341 .5000
END COMP

UF6 UO2F2 2 2938

READ PARAM $\overline{\mathrm{G}} \overline{\mathrm{G}}=203$ END PARAM

READ GEOM

CYLINDER $1110.16-89.6445$ - 149.3928

CYLINDER 6110.16 -80.8021 -149.3928

CYLINDER $0110.16-24.9172$-149.3928

CYLINDER $5110.6273-24.45$-149.86

CYLINDER $4117.22440-149.86$

CYLINDER $2117.781 .27-150.495$

CUBOID $3250-5050-501.27-180$

END GEOM

READ BNDS $X F C=V A C$ YFC $=V A C$ END BNDS

READ PLOT TTL=IUF6_UO2F2_2_938I

$P L T=N O P I C=M I X T U R E \bar{E} X U L=-50$ YUL $=00 Z Z L=5$

$X L R=50 \quad Y L R=00 \quad Z L R=-185$

$U A X=1$ WDN $=-1 \quad N A X=130 \quad L P I=8$

$\mathrm{NCH}=$ ! UFCWMS! END PLOT

END DATA

END

=CSAS25

UF6 UO2F2 21776

27GROUPNDF $\overline{4}$ INFHOMMEDIUM

UF6 11.03752939223597 .65922380 .8592234

1.5000

CARBONSTEEL 21.0

AL $30.05 .54-4 \quad$ END

CA $30.05 .30-3 \quad$ END

C $30.06 .59-3$ END

H 30.0 .0133 END

O 30.0 .0457 END

FE $30.03 .59-4$ END

MG $30.03 .25-3 \quad$ END

SI $30.08 .43-3$ END

NA $30.03 .89-4$ END

$S \quad 30.01 .09-4 \quad$ END

$\mathrm{H} 2 \mathrm{O} 41.0 \quad$ END

NI 50.0 .0603

CU 50.0 .02649

FE 50.0 .00119 END

MN $50.0 .0006 \quad$ END

SOLNUO2F2 642660 SPG $=5.86331 .0000293$

$9223597.65922380 .85922341 .5000 \quad$ END

END COMP

UF6 UO2F2 21776

READ PARAM $\bar{G}$ GEN $=203$ END PARAM

READ GEOM

CYLINDER $1110.16-99.6026-149.3928$

CYLINDER $6110.16-82.8499-149.3928$

CYLINDER $0110.16-24.9172-149.3928$

CYLINDER $5110.6273-24.45-149.86$

CYLINDER $4117.22440-149.86$

CYLINDER $2117.781 .27-150.495$

CUBOID $3250-5050-501.27-180$

END GEOM

READ BNDS XFC $=V A C$ YFC $=V A C$ END BNDS

READ PLOT TTL=!UF6 UO2F2_2 1776 !

PLT $=$ NO PIC $=M I X T U R E \bar{X} X U L=-50 \bar{Y} U L=00 Z U L=5$

$X L R=50$ YLR $=00 Z L R=-185$

$U A X=1$ WDN $=-1 \mathrm{NAX}=130 \mathrm{LPl}=8$

$\mathrm{NCH}=$ I UFCWMSIEND PLOT

END DATA

END 
=CSAS25

UF6 UO2F2 22825

27G $\bar{R} O U P N \bar{A} \overline{4}$ INFHOMMEDIUM

UF6 11.03752939223597 .65922380 .8592234

1.5000

CARBONSTEEL 21.0

AL $30.05 .54-4$

CA $30.05 .30-3$

C $30.06 .59-3$

H $\quad 30.0 .0133$

O 30.0 .0457

FE $30.03 .59-4$

MG $30.03 .25-3$

SI $30.08 .43-3$

NA $30.03 .89-4$

$S \quad 30.01 .09-4$

$\mathrm{H} 2 \mathrm{O} \quad 41.0$

N1 50.0 .0603

CU 50.0 .02649

FE 50.0 .00119

MN 50.0 .0006

SOLNUO2F2 $642660 \mathrm{SPG}=5.86331 .0000293$

9223597.65922380 .85922341 .5000 END

END COMP

UF6 UO2F2 22825

READ PARAM $\overline{\mathrm{G}} \overline{\mathrm{D}} \mathrm{N}=203$ END PARAM

READ GEOM

CYLINDER $1110.16-112.0501-149.3928$

CYLINDER $6110.16-85.4097-149.3928$

CYLINDER $0110.16-24.9172-149.3928$

CYLINDER $5110.6273-24.45-149.86$

CYLINDER $4117.22440-149.86$

CYLINDER $2117.781 .27-150.495$

CUBOID $3250-5050-501.27-180$

END GEOM

READ BNDS XFC=VAC YFC=VAC END BNDS

READ PLOT TTL=IUF6 UO2F2 22825 !

$P L T=N O P I C=M I X T U R E X U L=-50$ YUL $=00 \quad Z U L=5$

$X L R=50 \quad Y L R=00 Z L R=-185$

$U A X=1$ WDN $=-1 \quad N A X=130 \mathrm{LPI}=8$

$\mathrm{NCH}=$ ! UFCWMS! END PLOT

END DATA

END

-CSAS25

UF6_UO2F2_2_3873

27GROUPNDF- 4 INFHOMMEDIUM

UF6 11.03752939223597 .65922380 .8592234

1.5000

AL 30.0554 .4

CA $30.05 .30-3 \quad$ END

C $30.06 .59-3 \quad$ END

H 30.0 .0133 END

O 30.0 .0457 END

FE $30.03 .59-4 \quad$ END

MG $30.03 .25-3$ END

SI $30.08 .43-3 \quad$ END

NA $30.03 .89-4 \quad$ END

$S \quad 30.01 .09-4 \quad$ END

$\mathrm{H} 2 \mathrm{O} 41.0$ END

NI $50.0 .0603 \quad$ END

CU $50.0 .02649 \quad$ END
FE 50.0 .00119

END

MN 50.0 .0006

END

SOLNUO2F2 64266 O SPG $=5.86331 .0000293$

9223597.65922380 .85922341 .5000 END

END COMP

UF6 UO2F2 $2 \quad 3873$

READ PARAM $\overline{G E N}=203$ END PARAM

READ GEOM

CYLINDER $1110.16-124.4977-149.3928$

CYLINDER $6110.16 \quad-87.9694-149.3928$

CYLINDER $0110.16-24.9172-149.3928$

CYLINDER $5110.6273-24.45-149.86$

CYLINDER $4117.22440-149.86$

CYLINDER $2117.781 .27-150.495$

CUBOID $3250-5050-501.27-180$

END GEOM

READ BNDS XFC=VAC YFC=VAC END BNDS

READ PLOT TTL=IUF6 UO2F2 23873 !

$P L T=N O P I C=M I X T U R E X U L=-50$ YUL $=00 \quad Z U L=5$

$X L R=50$ YLR $=00 Z L R=-185$

$U A X=1$ WDN $=-1$ NAX $=130 \mathrm{LPI}=8$

$\mathrm{NCH}=$ ! UFCWMS! END PLOT

END DATA

END

$=$ CSAS25

UF6 UO2F2 24921

27GR̄OUPNDF $\overline{4}$ INFHOMMEDIUM

UF6 11.03752939223597 .65922380 .8592234

1.5000

CARBONSTEEL $21.0 \quad$ END

AL $30.05 .54-4$

CA $30.05 .30-3$

C $30.06 .59-3$

H 30.0 .0133

O $\quad 30.0 .0457$

FE $30.03 .59-4$

MG $30.03 .25-3$

SI $30.08 .43-3$

NA $30.03 .89-4$

$S \quad 30.01 .09-4$

$\mathrm{H} 2 \mathrm{O} 41.0$

NI 50.0 .0603

CU 50.0 .02649

FE 50.0 .00119

MN 50.0 .0006

END

END

END

END

END

END

END

END

END

END

END

END

END

END

END

SOLNUO2F2 642660 SPG $=5.86331 .0000293$

9223597.65922380 .85922341 .5000

END

END COMP

UF6 UO2F2 24921

REA $\bar{D}$ PARA $\bar{M} \bar{G} \bar{E} N=203$ END PARAM

READ GEOM

CYLINDER $1110.16-136.9452-149.3928$

CYLINDER $6110.16-90.5292-149.3928$

CYLINDER $0110.16-24.9172-149.3928$

CYLINDER $5110.6273-24.45-149.86$

CYLINDER $4117.22440-149.86$

CYLINDER $2117.781 .27-150.495$

CUBOID $3250-5050-50 \quad 1.27-180$

END GEOM

READ BNDS XFC=VAC YFC=VAC END BNDS

READ PLOT TTL=!UF6 UO2F2 24921 !

$P L T=N O P I C=M I X T U R E X U L=-50$ YUL $=00$ ZUL $=5$ $X L R=50$ YLR $=00$ ZLR $=-185$ 
$U A X=1 \quad W D N=-1 \quad N A X=130$ LPI $=8$

$\mathrm{NCH}=$ ! UFCWMSIEND PLOT

END DATA

END

\section{UF6 $(H / U=4)$}

\section{= CSAS25}

UF6 UO2F2 4_1036

27GROUPNDF $\overline{4}$ INFHOMMEDIUM

UF6 11.03752939223597 .65922380 .8492234 1.5100

END

CARBONSTEEL 21.0 END

AL $30.05 .54-4$

CA $30.05 .30-3$

C $30.06 .59-3$

H 30.0 .0133

C 30.0 .0457

FE 30.0 3.59-4

MG $30.03 .25-3$

SI $30.08 .43-3$

NA $30.03 .89-4$

S $30.01 .09-4$

$\mathrm{H} 2 \mathrm{O} \quad 41.0$

N1 50.0 .0603

CU 50.0 .02649

FE 50.0 .00119

MN 50.0 .0006

END

END

END

END

END

END

END

END

END

END

END

END

END

END

END

SOLNUO2F2 632520 SPG $=4.71831 .0000293$

9223597.65922380 .84922341 .5100 END

END COMP

UF6 UO2F2 41036

READ PARAM $\overline{\mathrm{G}} E \mathrm{~N}=203$ END PARAM

READ GEOM

CYLINDER $1110.16-84.6655-149.3928$

CYLINDER $6110.16 \quad-78.2542-149.3928$

CYLINDER $0110.16-24.9172-149.3928$

CYLINDER 5110.6273 -24.45 -149.86

CYLINDER $4117.22440-149.86$

CYLINDER $2117.781 .27-150.495$

CUBOID $3250-5050-501.27-180$

END GEOIN

READ BNDS XFC=VAC YFC $=V A C$ END BNDS

READ PLOT TTL=!UF6 UO2F2 41036 !

PLT $=$ NO PIC $=M I X T U R E$ XUL $=-50$ Y UL $=00$ ZUL $=5$

$X L R=50$ YLR $=00 Z L R=-185$

$U A X=1$ WDN $=-1 \quad N A X=130$ LPI $=8$

$\mathrm{NCH}=$ ! UFCWMSI END PLOT

END DATA

END

$=$ CSAS25

UF6_UO2F2_4_1246

27GROUPNDF $\overline{4}$ INFHOMMEDIUM

UF6 11.03752939223597 .65922380 .8492234

1.5100

CARBONSTEEL 21.0

END

AL $30.05 .54-4$

CA $30.05 .30-3$

C $30.06 .59-3$

H 30.0 .0133

O 30.0 .0457

FE $30.03 .59-4$

MG $30.03 .25-3$

END

END

END

END

END

END

END

END

SI $30.08 .43-3$
NA $30.03 .89-4$

S $30.01 .09-4$

$\mathrm{H} 2 \mathrm{O} 41.0$

NI 50.0 .0603

CU 50.0 .02649

FE 50.0 .00119

MN 50.0 .0006

END

END

END

END

END

END

END

SOLNUO2F2 632520 SPG $=4.71831 .0000293$

9223597.65922380 .84922341 .5100 END

END COMP

UF6 UO2F2 $8 \quad 1246$

READ PARA $\bar{M} \bar{G} E N=203$ END PARAM

READ GEOM

CYLINDER $1110.16-85.9102$ - 149.3928

CYLINDER $6110.16-78.2019-149.3928$

CYLINDER $0110.16-24.9172-149.3928$

CYLINDER $5110.6273-24.45-149.86$

CYLINDER $4117.22440-149.86$

CYLINDER $21 \quad 17.781 .27-150.495$

CUBOID $3250-5050-501.27-180$

END GEOM

READ BNDS XFC $=V A C$ YFC $=V A C E N D$ BNDS

READ PLOT TTL=IUF6 UO2F2 4 1246 I

$P L T=N O P I C=M I X T U R E X U L=-50$ Y $U L=00 \quad Z U L=5$

$X I R=50$ YLR $=00$ ZLR $=-185$

$U A X=1$ WDN $=-1 \quad N A X=130 \quad L P \mid=8$

$\mathrm{NCH}=$ I UFCWMSIEND PLOT

END DATA

END

- CSAS25

UF6 UO2F2 41875

27GR̄OUPNDF $\overline{4}$ INFHOMMEDIUM

UF6 11.03752939223597 .65922380 .8492234

1.5100

CARBONSTEEL 21.0

END

AL $30.05 .54-4$

CA $30.05 .30-3$

C $30.06 .59-3$

H 30.0 .0133

O 30.0 .0457

FE 30.0 3.59-4

MG $30.03 .25-3$

SI $30.08 .43-3$

NA $30.03 .89-4$

S $30.01 .09-4$

$\mathrm{H} 2 \mathrm{O} 41.0$

NI 50.0 .0603

CU 50.0 .02649

FE 50.0 .00119

MN 50.0 .0006

END

END

END

END

END

END

END

END

END

END

END

END

END

END

END

END

SOLNUO2F2 632520 SPG $=4.71831 .0000293$

9223597.65922380 .84922341 .5100 END

END COMP

UF6_UO2F2_8_1875

READ PARAM $\bar{G} E N=203$ END PARAM

READ GEOM

CYLINDER $1110.16-89.6445-149.3928$

CYLINDER $6110.16-78.0448-149.3928$

CYLINDER $0110.16-24.9172-149.3928$

CYLINDER $5110.6273-24.45-149.86$

CYLINDER $4117.22440-149.86$

CYLINDER $2117.781 .27-150.495$

CUBOID $3250-5050-501.27-180$ 
END GEOM

READ BNDS XFC $=V A C$ YFC $=V A C$ END BNDS

READ PLOT TTL=IUF6 UO2F2 4 1875 I

$P L T=N O P I C=M I X T U R E X U L=-50 \quad \bar{Y} U L=00 Z Z L=5$

$X L R=50$ YLR $=00$ ZLR $=-185$

$U A X=1$ WDN $=-1 \quad N A X=130 \quad L P I=8$

$N C H=!$ UFCWMSI END PLOT

END DATA

END

\section{- CSAS25}

UF6 UO2F2 43553

27GR̄OUPNDF $\overline{4}$ INFHOMMEDIUM

UF6 11.03752939223597 .65922380 .8492234

1.5100

CARBONSTEEL 21.0

AL $30.05 .54-4$

CA $30.05 .30-3$

C $30.06 .59-3$

H 30.0 .0133

O 30.0 .0457

FE $30.03 .59-4$

MG $30.03 .25-3$

SI $30.08 .43-3$

NA $30.03 .89-4$

S $30.01 .09-4$

H2O 41.0

NI 50.0 .0603

CU 50.0 .02649

FE 50.0 .00119

MN 50.0 .0006

SOLNUO2F2 632520 SPG $=4.71831 .0000293$

9223597.65922380 .84922341 .5100 END

END COMP

UF6 UO2F2 43553

READ PARA $\bar{M} \bar{G}=N=203$ END PARAM

READ GEOM

CYLINDER $1110.16-99.6026-149.3928$

CYLINDER 6110.16 -77.6260 -149.3928

CYLINDER $0110.16-24.9172-149.3928$

CYLINDER $5110.6273-24.45-149.86$

CYLINDER $4117.22440-149.86$

CYLINDER $2117.781 .27-150.495$

CUBOID $3250-5050-501.27-180$

END GEOM

READ BNDS $X F C=V A C$ YFC $=V A C$ END BNDS

READ PLOT TTL=!UF6 UO2F2 43553 !

$P L T=N O P I C=M I X T U R E X U L=-50$ YUL $=00$ ZUL $=5$

$X L R=50$ YLR $=00$ ZLR $=-185$

$U A X=1$ WDN $=-1$ NAX $=130$ LPI $=8$

$\mathrm{NCH}=$ ! UFCWMS! END PLOT

END DATA

END

$=$ CSAS25

UF6 UO2F2 4 5649

27GR̄OUPNDF $\overline{4}$ INFHOMMEDIUM

UF6 11.03752939223597 .65922380 .8492234 1.5100

CARBONSTEEL 21.0

AL $30.05 .54-4$

CA $30.05 .30-3$

C 30.06 .593

H 30.0 .0133
O 30.0 .0457

FE $30.03 .59-4$

MG $30.03 .25-3$

SI $30.08 .43-3$

NA $30.03 .89-4$

S $30.01 .09-4$

H2O 41.0

N1 50.0 .0603

CU 50.0 .02649

FE 50.0 .00119

MN 50.0 .0006

SOLNUO2F2 632520 SPG $=4.71831 .0000293$ 9223597.65922380 .84922341 .5100 END

END COMP

UF6 UO2F2 45649

REA $\bar{D}$ PARAM $\overline{\mathrm{G}} \overline{\mathrm{G}} \mathrm{N}=203$ END PARAM

READ GEOM

CYLINDER $1110.16-112.0501-149.3928$

CYLINDER $6110.16-77.1024-149.3928$

CYLINDER $0110.16-24.9172-149.3928$

CYLINDER 51 10.6273 -24.45 -149.86

CYLINDER $4117.22440-149.86$

CYLINDER $2117.781 .27-150.495$

CUBOID $3250-5050-50 \quad 1.27-180$

END GEOM

READ BNDS XFC $=V A C$ YFC $=V A C$ END BNDS

READ i -OT TTL=!UF6_UO2F2_4_5649!

$P L T=N O P I C=M I X T U R E X U L=-50$ YUL $=00 Z Z L=5$

$X L R=50$ YLR $=00 Z L R=-185$

$U A X=1$ WDN $=-1 \quad N A X=130 \quad L P I=8$

$\mathrm{NCH}=$ ! UFCWMS! END PLOT

END DATA

END

- CSAS25

UF6 UO2F2 4 47746

27GROUPNDF $\overline{4}$ INFHOMMEDIUM

UF6 11.03752939223597 .65922380 .8492234 1.5100

CARBONSTEEL 21.0

AL $30.05 .54-4$

CA $30.05 .30-3$

C $30.06 .59-3$

H 30.0 .0133

O 30.0 .0457

FE $30.03 .59-4$

MG $30.03 .25-3$

SI $30.08 .43-3$

NA $30.03 .89-4$

S $30.01 .09-4$

$\mathrm{H} 2 \mathrm{O} 41.0$

NI 50.0 .0603

CU 50.0 .02649

FE 50.0 .00119

MN 50.0 .0006

END

END

END

END

END

END

END

END

END

END

END

END

END

END

END

END

END

SOLNUO2F2 632520 SPG $=4.71831 .0000293$

9223597.65922380 .84922341 .5100 END

END COMP

UF6 UO2F2 47746

REA $\bar{D}$ PARA $\bar{M} \bar{G} E N=203$ END PARAM

READ GEOM

CYLINDER $1110.16-124.4977-149.3928$

CYLINDER $6110.16-76.5788-149.3928$

CYLINDER $0110.16-24.9172-149.3928$ 
CYLINDER 51 10.6273 -24.45 -149.86

CYLINDER $4117.22440-149.86$

CYLINDER $2117.781 .27-150.495$

CUBOID $3250-5050-501.27-180$

END GEOM

READ BNDS XFC $=V A C$ YFC $=V A C$ END BNDS

READ PLOT TTLIUF6 UO2F2 477461

PLT=NO PIC $=M I X T U R E X U L=-50$ YUL $=00 Z Z L=5$

$X L R=50$ YLR $=00 Z L R=-185$

$U A X=1$ WDN $=-1 \quad N A X=130 \mathrm{LPI}=8$

$\mathrm{NCH}=$ ! UFCWMS! END PLOT

END DATA

END

\section{= CSAS25}

UF6 UO2F2 49843

27GROUPNDF $\overline{4}$ INFHOMMEDIUM

UF6 11.03752939223597 .65922380 .8492234

1.5100

END

CARBONSTEEL 21.0 END

AL $30.05 .54-4$

CA $30.05 .30-3$

C $30.06 .59-3$

END

H 30.0 .0133

O 30.0 .0457

FE $30.03 .59-4$

MG $30.03 .25-3$

SI $30.08 .43-3$

NA $30.03 .89-4$

END

END

END

END

END

END

END

END

S $30.01 .09-4$

END

$\mathrm{H} 2 \mathrm{O} 41.0$

NI 50.0 .0603

CU 50.0 .02649

END

END

END

END

FE 50.0 .00119

SOLNUO2F2 $632520 \mathrm{SPG}=4.71831 .0000293$

9223597.65922380 .84922341 .5100 END

END COMP

UF6_UO2F2 4 _9843

READ PARAM $\overline{\mathrm{G}} \overline{\mathrm{B}} \mathrm{N}=203 \mathrm{END}$ PARAM

READ GEOM

CYLINDER $1110.16-136.9452-149.3928$

CYLINDER $6110.16 \quad-76.0553-149.3928$

CYLINDER $0110.16-24.9172-149.3928$

CYLINDER $5110.6273-24.45$-149.86

CYLINDER $4117.22440-149.86$

CYLINDER $2117.781 .27-150.495$

CUBOID $3250-5050-501.27-180$

END GEOM

READ BNDS XFC $=$ VAC YFC $=V A C$ END BNDS

READ PLOT TTL $=$ !UF6 9843 !

$P L T=N O P I C=M I X T U R E$ XUL $=-50 \quad Y U L=00 \quad Z U L=5$

$X L R=50 Y L R=00 Z L R=-185$

$U A X=1$ WDN $=-1$ NAX $=130$ LPI $=8$

$\mathrm{NCH}=$ !UFCWMS! END PLOT

END DATA

END

\section{UF6 $(H / U=8)$}

= CSAS25

UF6 UO2F2 82073

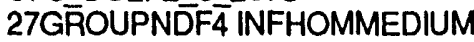

UF6 11.03752939223597 .65922380 .8492234 1.5100

CARBONSTEEL $21.0 \quad$ END

AL $30.05 .54-4$

CA $30.05 .30-3$

C $30.06 .59-3$

H 30.0 .0133

O 30.0 .0457

FE $30.03 .59-4$

MG $30.03 .25-3$

SI $30.08 .43-3$

NA $30.03 .89-4$

S $30.01 .09-4$

$\mathrm{H} 2 \mathrm{O} \quad 41.0$

NI 50.0 .0603

CU 50.0 .02649

FE 50.0 .00119

MN 50.0 .0006

END

END

END

END

END

END

END

END

END

END

END

END

END

END

END

SOLNUO2F2 621700 SPG $=3.48131 .0000293$

9223597.65922380 .84922341 .5100 END

END COMP

UF6_UO2F2_8_2073

READ PARAM GEN=203 END PARAM

READ GEOM

CYLINDER $1110.16-84.6655$-149.3928

CYLINDER $6110.16-75.0582-149.3928$

CYLINDER $0110.16-24.9172-149.3928$

CYLINDER $5110.6273-24.45-149.86$

CYLINDER $4117.22440-149.86$

CYLINDER $2117.781 .27-150.495$

CUBOID $3250-5050-501.27-180$

END GEOM

READ BNDS XFC-VAC YFC-VAC END BNDS

READ PLOT TTL=!UF6 UO2F2 42073 !

$P L T=N O P I C=M I X T U R E \bar{E} X U L=-50$ YUL $=00$ ZUL $=5$

$X L R=50$ YLR $=00$ ZLR $=-185$

$U A X=1$ WDN $=-1 \quad N A X=130 \quad \mathrm{LPl}=8$

$\mathrm{NCH}=$ ! UFCWMS! END PLOT

END DATA

END

- CSAS25

UF6_UO2F2_8_2492

27GR'OUPNDF $\overline{4}$ INFHOMMEDIUM

UF6 11.03752939223597 .65922380 .8492234

1.5100

CARBONSTEEL 21.0

AL $30.05 .54-4$

CA $30.05 .30-3$

C $30.06 .59-3$

H 30.0 .0133

O 30.0 .0457

FE $30.03 .59-4$

MG $30.03 .25-3$

SI $30.08 .43-3$

NA $30.03 .89-4$

S $30.01 .09-4$

$\mathrm{H} 2 \mathrm{O} \quad 41.0$

N1 50.0 .0603

CU 50.0 .02649

FE 50.0 .00119

MN 50.0 .0006

END

END

END

END

END

END

END

END

END

END

END

END

END

END

END

END

END

SOLNUO2F2 621700 SPG=3.4813 1.0000293

9223597.65922380 .84922341 .5100 END 
END COMP

UF6_UO2F2_8_2492

READ PARA $\bar{M} \bar{G} \overline{\mathrm{G}}=203$ END PARAM

READ GEOM

CYLINDER $1110.16-85.9102-149.3928$

CYLINDER $6110.16 \quad-74.3593-149.3928$

CYLINDER $0110.16-24.9172-149.3928$

CYLINDER 51 10.6273 -24.45 - 149.86

CYLINDER $4117.22440-149.86$

CYLINDER $2117.781 .27-150.495$

CUBOID $3250-5050-50 \quad 1.27-180$

END GEOM

READ BNDS XFC=VAC YFC=VAC END BNDS

READ PLOT TTL=IUF6 UO2F2 4 2492 I

$P L T=N O P I C=M I X T U R E X U L=50$ YUL $=00 Z Z L=5$

$X L R=50$ YLR $=00 \quad Z L R=-185$

$U A X=1$ WDN $=-1 \quad N A X=130 \quad \mathrm{LPI}=8$

$\mathrm{NCH}=$ ! UFCWMSI END PLOT

END DATA

END

$=$ CSAS25

UF6_UO2F2 8 3750

27GRROUPNDF $\overline{4}$ INFHOMMEDIUM

UF6 11.03752939223597 .65922380 .8492234

1.5100

CARBONSTEEL 21.0

AL $30.05 .54-4$

CA $30.05 .30-3$

C $30.06 .59-3$

H 30.0 .0133

O 30.0 .0457

FE $30.03 .59-4$

MG $30.03 .25-3$

SI $30.08 .43-3$

NA $30.03 .89-4$

S $30.01 .09-4$

$\mathrm{H} 2 \mathrm{O} \quad 41.0$

NI 50.0 .0603

CU 50.0 .02649

FE 50.0 .00119

MN 50.0 .0006

END

END

END

END

END

END

END

END

END

END

END

END

END

END

END

END

END

SOLNUO2F2 621700 SPG $=3.48131 .0000293$ 9223597.65922380 .84922341 .5100 END

END COMP

UF6 UO2F2 83750

READ PARAM $\overline{\mathrm{G}} \overline{\mathrm{D}} \mathrm{N}=203$ END PARAM

READ GEOM

CYLINDER $1110.16-89.6445-149.3928$

CYLINDER $6110.16-72.2624-149.3928$

CYLINDER $0110.16-24.9172-149.3928$

CYLINDER 5110.6273 -24.45 -149.86

CYLINDER $4117.22440-149.86$

CYLINDER $2117.781 .27-150.495$

CUBOID $3250 \quad-5050-501.27-180$

END GEOM

READ BNDS $X F C=V A C$ YFC $=V A C$ END BNDS

READ PLOT TTL=!UF6 UO2F2 43750 !

$P L T=N O P I C=M \mid X T U R E X U L=-50 \quad$ YUL $=00 \quad Z U L=5$

$X L R=50$ YLR $=00 Z Z R=-185$

$\cup A X=1$ WDN $=-1 \quad N A X=130 \quad L P \mid=8$

$\mathrm{NCH}=\mid$ UFCWMSIEND PLOT

END DATA

END
$=$ CSAS25

UF6 UO2F2 87105

27GR̄OUPNDF $\overline{4}$ INFHOMMEDIUM

UF6 11.0375293922359765922380 .8492234

1.5100

CARBONSTEEL 21.0

END

AL $30.05 .54-4$

CA $30.05 .30-3$

C $30.06 .59-3$

H 30.0 .0133

O 30.0 .0457

FE $30.03 .59-4$

MG $30.03 .25-3$

SI $30.08 .43-3$

NA $30.03 .89-4$

$S \quad 30.01 .09-4$

$\mathrm{H} 2 \mathrm{O} \quad 41.0$

NI 50.0 .0603

CU 50.0 .02649

FE 50.0 .00119

MN 50.0 .0006

END

END

END

END

END

END

END

END

END

END

END

END

END

END

END

END

SOLNUO2F2 621700 SPG=3.4813 1.0000293

9223597.65922380 .84922341 .5100 END

END COMP

UF6 UO2F2 87105

READ PARAM $\bar{G} E N=203$ END PARAM

READ GEOM

CYLINDER $1110.16-99.6026-149.3928$

CYLINDER $6110.16-66.6706-149.3928$

CYLINDER $0110.16-24.9172-149.3928$

CYLINDER $5110.6273-24.45-149.86$

CYLINDER $4117.22440-149.86$

CYLINDER $2117.781 .27-150.495$

CUBOID $3250-5050-501.27-180$

END GEOM

READ BNDS XFC=VAC YFC $=V A C$ END BNDS

READ PLOT TTL=!UF6 UO2F2 47105

$P L T=N O P I C=M I X T U R E X U L=-50$ YUL $=00 \quad Z U L=5$

$X L R=50$ YLR $=00 Z L R=-185$

$U A X=1$ WDN $=-1 \quad N A X=130 \quad L P I=8$

$\mathrm{NCH}=$ ! UFCWMS! END PLOT

END DATA

END

= CSAS25

UF6_UO2F2_8_11299

27GR'OUPNDF 4 INFHOMMEDIUM

UF6 11.03752939223597 .65922380 .8492234 1.5100

CARBONSTEEL 21.0

AL $30.05 .54-4$

CA $30.05 .30-3$

C $30.06 .59-3$

H 30.0 .0133

O 30.0 .0457

FE $30.03 .59-4$

MG $30.03 .25-3$

SI $30.08 .43-3$

NA $30.03 .89-4$

$S \quad 30.01 .09-4$

$\mathrm{H} 2 \mathrm{O} \quad 41.0$

NI 50.0 .0603

CU 50.0 .02649

END

END

END

END

END

END

END

END

END

END

END

END

END

END

END 
$\begin{array}{ll}\text { FE } 50.0 .00119 & \text { END } \\ \text { MN } 50.0 .0006 & \text { END }\end{array}$

SOLNUO2F2 621700 SPG $=3.48131 .0000293$

9223597.65922380 .84922341 .5100 END

END COMP

UF6 UO2F2 811299

READ PARA $\bar{M} \bar{G} E N=203$ END PARAM

READ GEOM

CYLINDER $1110.16 \cdot 112.0501-149.3928$

CYLINDER $6110.16-59.6810-149.3928$

CYLINDER $0110.16-24.9172$-149.3928

CYLINDER $5110.6273-24.45-149.86$

CYLINDER $4117.22440-149.86$

CYLINDER $2117.781 .27-150.495$

CUBOID $3250-5050-501.27-180$

END GEOM

READ BNDS XFC $=V A C$ YFC $=V A C$ END BNDS

READ PLOT TTL=!UF6 UO2F2 4 11299 !

$P L T=N O P I C=M I X T U R E X U L=-50 \bar{Y} U L=00 Z U L=5$

$X L R=50$ YLR $=00$ ZLR $=-185$

$U A X=1$ WDN $=-1$ NAX $=130$ LPI $=: 8$

$\mathrm{NCH}=$ ! UFCWMS! END PLOT

END DATA

END

- CSAS25

UF6 UO2F2 8 _ 15492

27G $\bar{R} O U P N \bar{F} \overline{4}$ INFHOMMEDIUM

UF6 11.03752939223597 .65922380 .8492234

1.5100

CARBONSTEEL 21.0 END

AL $30.05 .54-4$

CA $30.05 .30-3$

C $30.06 .59-3$

H 30.0 .0133

O 30.0 .0457

FE $30.03 .59-4$

MG $30.03 .25-3$

SI $30.08 .43-3$

NA $30.03 .89-4$

S $30.01 .09-4$

$\mathrm{H} 2 \mathrm{O} \quad 41.0$

NI 50.0 .0603

CU 50.0 .02649

FE 50.0 .00119

MN 50.0 .0006

END

END

END

END

END

END

END

END

END

END

END

END

END

END

END

SOLNUO2F2 $621700 \mathrm{SPG}=3.48131 .0000293$

9223597.65922380 .84922341 .5100 END

END COMP

UF6 UO2F2 $8 \quad 15492$

READ PARAM $\overline{\mathrm{G}} \overline{\mathrm{G}} \mathrm{N}=203$ END PARAM

READ GEOM

CYLINDER $1110.16-124.4977-149.3928$

CYLINDER $6110.16-52.6913-149.3928$

CYLINDER $0110.16-24.9172-149.3928$

CYLINDER 5110.6273 -24.45 -149.86

CYLINDER $4117.22440-149.86$

CYLINDER $2117.781 .27-150.495$

CUBOID $3250-5050-501.27-180$

END GEOM

READ BNDS XFC $=V A C$ YFC $=V A C$ END BNDS

READ PLOT TTL=!UF6 UO2F2_4_15492!

$P L T=N O P I C=M I X T U R E X U L=-50$ YUL $=00 Z U L=5$

$X L R=50$ YLR $=00 Z L R=-185$
$U A X=1$ WDN $=-1 \quad N A X=130 \quad L P I=8$

$\mathrm{NCH}=\mid$ UFCWMS! END PLOT

END DATA

END

- CSAS25

UF6_UO2F2_8_19686

27G $\bar{R}$ OUPNDF $\overline{4}$ INFHOMMEDIUM

UF6 11.03752939223597 .65922380 .8492234 1.5100

CARBONSTEEL 21.0

Al. $30.05 .54-4$

CA $30.05 .30-3$

C $30.06 .59-3$

H 30.0 .0133

O $\quad 30.0 .0457$

FE $30.03 .59-4$

MG $30.03 .25-3$

SI $\quad 30.08 .43-3$

NA $30.03 .89-4$

S $30.01 .09-4$

$\mathrm{H} 2 \mathrm{O} \quad 41.0$

NI 50.0 .0603

CU 50.0 .02649

FE 50.0 .00119

MN 50.0 .0006

END

END

END

END

END

END

END

END

END

END

END

END

END

END

END

END

END

SOLNUO2F2 62.1700 SPG $=3.48131 .0000293$

9223597.65922380 .84922341 .5100 END

END COMP

UF6 UO2F2 819686

READ PARA $\bar{M} \overline{G E N}=203$ END PARAM

READ GEOM

CYLINDER $1110.16-136.9452-149.3928$

CYLINDER $6110.16-45.7016-149.3928$

CYLINDER $0110.16-24.9172$ - 149.3928

CYLINDER $5110.6273-24.45-149.86$

CYLINDER $4117.22440-149.86$

CYLINDER $2117.781 .27-150.495$

CUBOID $3250-5050-501.27-180$

END GEOM

READ BNDS XFC=VAC YFC=VAC END BNDS

READ PLOT TTL=IUF6 19686 !

$P L T=N O P I C=M I X T U R E \bar{X}$ XUL $=-50$ YUL $=00$ ZUL $=5$

$X L R=50 Y L R=00 Z L R=-185$

$U A X=1 W D N=-1 \quad N A X=130 \quad L P \mid=8$

$\mathrm{NCH}=$ ! UFCWMS! END PLOT

END DATA

END

UF6 $(H / U=2)$, DRY RECEPT.

$=$ CSAS25

UF6 UO2F2 2 _O

27GR̄OUPNDFF $\overline{4}$ INFHOMMEDIUM

UF6 11.03752939223597 .65922380 .8592234

1.5000

CARBONSTEEL 21.0

AL $30.05 .54-4$

CA $30.05 .30-3$

C $30.06 .59-3$

H 30.0 .0133

O 30.0 .0457

FE $30.03 .59-4$

MG $30.03 .25-3$

END

END

END

END

END

END

END

END

END 


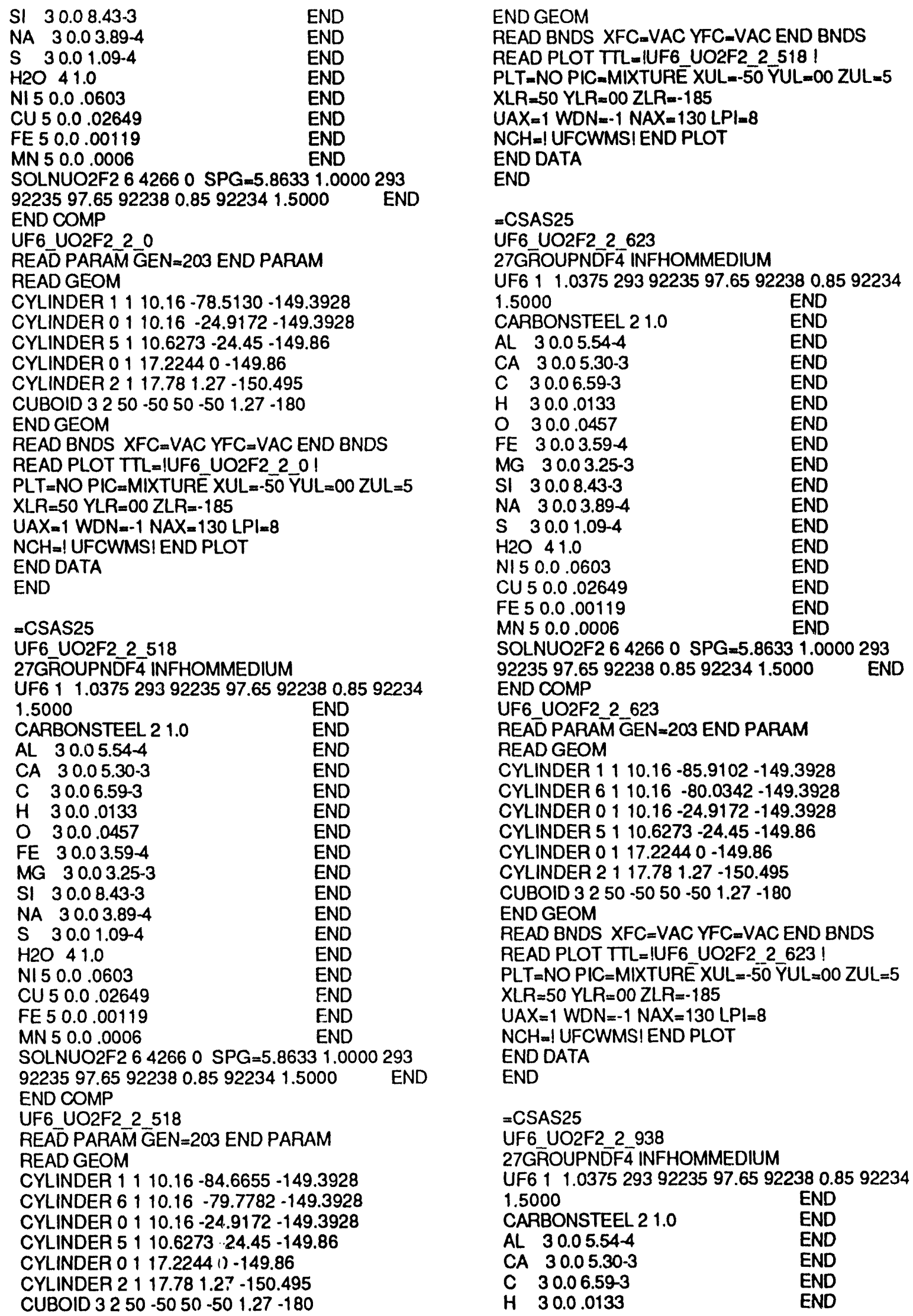




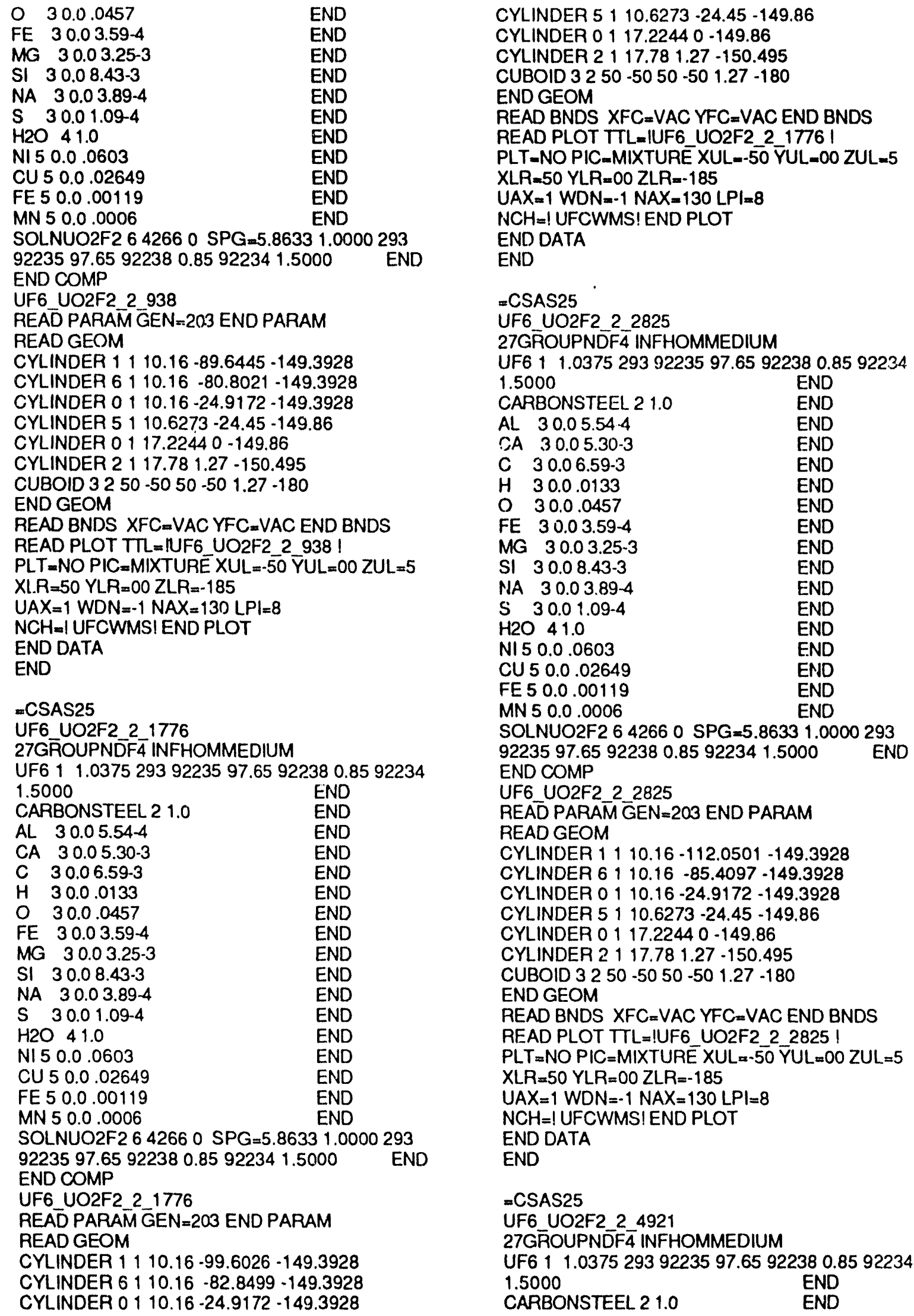




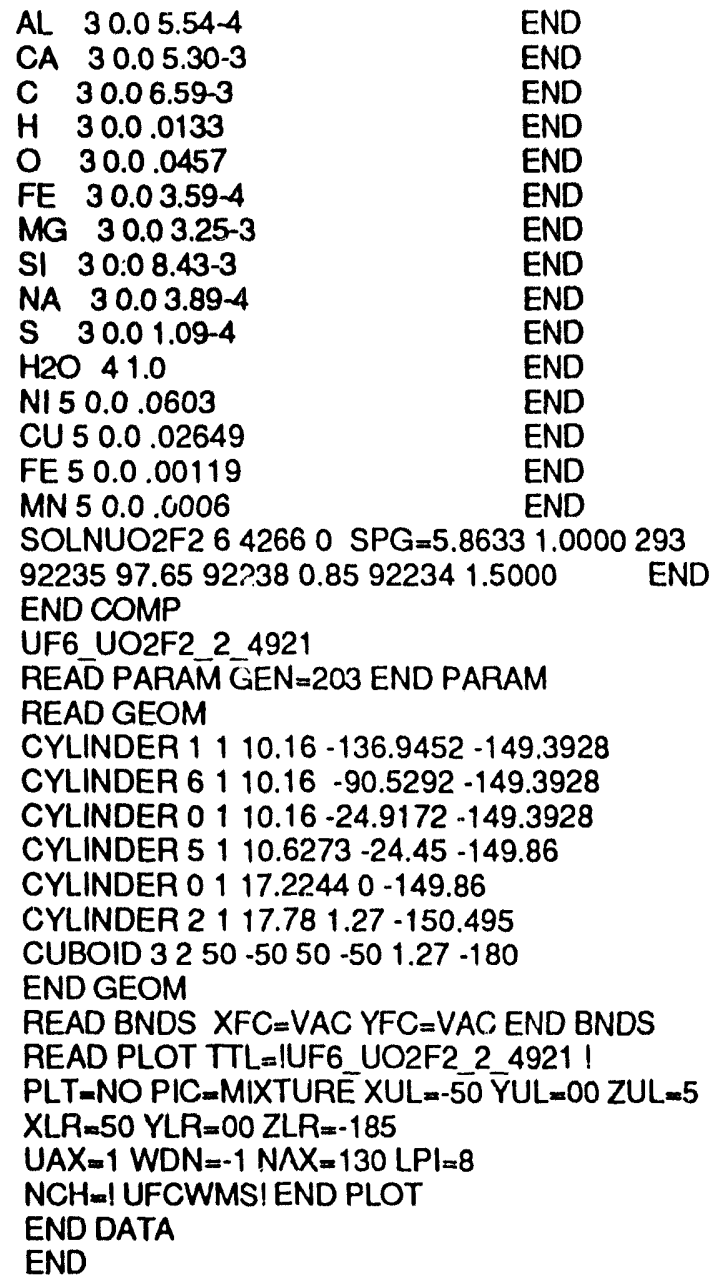

UF6 $(H / U=4)$, DRY RECEPT.

= CSAS25

UF6 UO2F2 $4 \quad 1036$

27GROUPNDF $\overline{4}$ INFHOMMEDIUM

UF6 11.03752939223597 .65922380 .8492234 1.5100

CARBONSTEEL 21.0

END

AL $30.05 .54-4$

CA $30.05 .30-3$

C $30.06 .59-3$

H 30.0 .0133

O 30.0 .0457

FE $30.03 .59-4$

MG $30.03 .25-3$

SI $30.08 .43-3$

NA $30.03 .89-4$

S $30.01 .09-4$

$\mathrm{H} 2 \mathrm{O} \quad 41.0$

NI 50.0 .0603

CU 50.0 .02649

FE 50.0 .00119

MN 50.0 .0006

END

END

END

END

END

END

END

END

END

END

END

END

END

END

END

END

SOLNUO2F2 632520 SPG $=4.71831 .0000293$ 9223597.65922380 .84922341 .5100 END END COMP
UF6_UO2F2_4_1036

REA $\bar{D}$ PARA $\bar{M} \bar{G} E N=203$ END PARAM

READ GEOM

CYLINDER 1110.16 -84.6655 - 149.3928

CYLINDER $6110.16 \quad-78.2542-149.3928$

CYLINDER $0110.16-24.9172-149.3928$

CYLINDER $5110.6273-24.45-149.86$

CYLINDER $0117.22440-149.86$

CYLINDER $2117.781 .27-150.495$

CUBOID $3250-5050-50 \quad 1.27-180$

END GEOM

READ BNDS XFC=VAC YFC $=V A C$ END BNDS

READ PLOT TTL=IUFG_UO2F2_4_1036!

$P L T=N O P I C=M I X T U R E X U L=-50 \quad \bar{Y} U L=00 Z U L=5$

$X L R=50$ YLR $=00 Z L R=-185$

$U A X=1 W D N=-1 N A X=130 L P \mid=8$

$\mathrm{NCH}=$ ! UFCWMSI END PLOT

END DATA

END

- CSAS25

UF6 UO2F2 41246

27GR̄OUPNDF $\overline{4}$ INFHOMMEDIUM

UF6 11.03752939223597 .65922380 .8492234

1.5100

CARBONSTEEL 21.0

AL $30.05 .54-4$

CA $30.05 .30-3$

C $30.06 .59-3$

H 30.0 .0133

O 30.0 .0457

FE $30.03 .59-4$

MG $30.03 .25-3$

SI $30.08 .43-3$

NA $30.03 .89-4$

S $30.01 .09-4$

$\mathrm{H} 2 \mathrm{O} 41.0$

NI 50.0 .0603

CU 50.0 .02649

FE 50.0 .00119

MN 50.0 .0006

END

END

END

END

END

END

END

END

END

END

END

END

END

END

END

END

END

SOLNUO2F2 632520 SPG $=4.71831 .0000293$

9223597.65922380 .84922341 .5100 END

END COMP

UF6 UO2F2 $8 \quad 1246$

READ PARA $\bar{M} \overline{\mathrm{G}} \overline{\mathrm{D}}=203$ END PARAM

READ GEOM

CYLINDER $1110.16-85.9102-149.3928$

CYLINDER $6110.16 \quad-78.2019-149.3928$

CYLINDER $0110.16-24.9172-149.3928$

CYLINDER $51 \quad 10.6273-24.45-149.86$

CYLINDER $0117.22440-149.86$

CYLINDER $2117.781 .27-150.495$

CUBOID $3250-5050-501.27-180$

END GEOM

READ BNDS XFC $=V A C$ YFC $=V A C$ END BNDS

READ PLOT TTL=IUF6 UO2F2 4 1246 ।

PLT $=$ NO PIC $=M I X T U R E X U L=-50$ YUL $=00 Z U L=5$

$X L R=50$ YLR $=00 Z L R=-185$

$U A X=1$ WDN $=-1 \quad N A X=130$ LPI $=8$

$\mathrm{NCH}=$ ! UFCWMS! END PLOT

END DATA

END 
$=$ CSAS 25

UF6_UO2F2_4_3553

27GROUPNDFA INFHOMMEDIUM

UF6 11.03752939223597 .65922380 .8492234

1.5100

CARBONSTEEL 21.0

AL $30.05 .54-4$

CA $30.05 .30-3$

C $30.06 .59-3$

H 30.0 .0133

O 30.0 .0457

FE $30.03 .59-4$

MG $30.03 .25-3$

SI $30.08 .43-3$

NA $30.03 .89-4$

S $30.01 .09-4$

$\mathrm{H} 2 \mathrm{O} \quad 41.0$

NI 50.0 .0603

CU 50.0 .02649

FE 50.0 .00119

MN 50.0 .0006

SOLNUO2F2 632520 SPG $=4.71831 .0000293$

9223597.65922380 .84922341 .5100 END

END COMP

UF6_UO2F2_4_3553

READ PARAM $\bar{M} \bar{G} E N=203$ END PARAM

READ GEOM

CYLINDER $1110.16-99.6026-149.3928$

CYLINDER $6110.16-77.6260-149.3928$

CYLINDER $0110.16-24.9172-149.3928$

CYLINDER 5 : $10.6273-24.45-149.86$

CYLINDER $4117.22440-149.86$

CYLINDER $2117.781 .27-150.495$

CUBOID $3250-5050-501.27-180$

END GEOM

READ BNDS XFC=VAC YFC=VAC END BNDS

READ PLOT TTL=!UF6 UO2F2 4 3553 !

$P L T=N O P I C=M I X T U R E X U L=-50$ YUL $=00 \quad Z U L=5$

$X L R=50$ YLR $=00 Z Z R=-185$

$U A X=1$ WDN $=-1$ NAX $=130 \angle P \mid=8$

$\mathrm{NCH}=$ ! UFCWMS! END PLOT

END DATA

END

$=$ CSAS25

UF6 UO2F2 45649

27GR $\bar{R}$ OUPNDF $\overline{4}$ INFHOMMEDIUM

UF6 11.03752939223597 .65922380 .8492234

1.5100

CARBONSTEEL 21.0

END

AL $30.05 .54-4$

CA $30.05 .30-3$

C $30.06 .59-3$

H 30.0 .0133

O 30.0 .0457

FE $30.03 .59-4$

MG $30.03 .25-3$

SI $30.08 .43-3$

NA $30.03 .89-4$

S $30.01 .09-4$

$\mathrm{H} 2 \mathrm{O} \quad 41.0$

NI 0.0 .0603

CU 50.0 .02649

FE 50.0 .00119
MN 50.0 .0006

END

SOLNUO2F2 632520 SPG $=4.71831 .0000293$

9223597.65922380 .84922341 .5100 END

END COMP

UF6 UO2F2 45649

READ PARA $\bar{M} \bar{G} E N=203$ END PARAM

READ GEOM

CYLINDER 1110.16 - $112.0501-149.3928$

CYLINDER $6110.16-77.1024-149.3928$

CYLINDER $0110.16-24.9172-149.3928$

CYLINDER $5110.6273-24.45-149.86$

CYLINDER $0117.22440-149.86$

CYLINDER $2117.781 .27-150.495$

CUBOID $3250-5050-50 \quad 1.27-180$

END GEOM

READ BNDS XFC=VAC YFC=VAC END BNDS

READ PLOT TTL=IUF6_UO2F2_4_5649!

$P L T=N O P I C=M I X T U R E$ YUL $=-50$ YUL $=00 \mathrm{ZUL}=5$

$X L R=50$ YLR $=00 Z L R=-185$

$U A X=1$ WDN $=-1 \quad N A X=130 \quad \mathrm{LPI}=8$

$\mathrm{NCH}=$ ! UFCWMS! END PLOT

END DATA

END

= CSAS25

UF6 UO2F2 47746

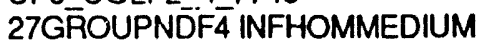

UF6 11.03752939223597 .65922380 .8492234

\subsection{0}

CARBONSTEEL 21.0

AL 30.05 .54 .4

CA $30.05 .30-3$

C $30.06 .59-3$

H 30.0 .0133

O 30.0 .0457

FE $30.03 .59-4$

MG $30.03 .25-3$

SI $30.08 .43-3$

NA $30.03 .89-4$

S $30.01 .09-4$

$\mathrm{H} 2 \mathrm{O} 41.0$

N1 50.0 .0603

CU 50.0 .02649

FE 50.0 .00119

MN 50.0 .0006

END

END

END

END

END

END

END

END

END

END

END

END

END

END

END

END

END

SOLNUO2F2 632520 SPG $=4.71831 .0000293$

9223597.65922380 .84922341 .5100 END

END COMP

UF6_UO2F2_4_7746

READ PARA $\bar{M} \bar{G} E N=203$ END PARAM

READ GEOM

CYLINDER $1110.16-124.4977$-149.3928

CYLINDER $6,10.16-76.5788-149.3928$

CYLINDER $0110.16-24.9172-149.3928$

CYLINDER $5110.6273-24.45-149.86$

CYLINDER $0117.22440-149.86$

CYLINDER $2117.781 .27-150.495$

CUBOID $3250-5050-501.27-180$

END GEOM

READ BNDS XFC $=V A C$ YFC $=V A C$ END BNDS

READ PLOT TTL=IUF6 UO2F2 4 7746 !

PLT=NO PIC=MIXTURE XUL $=-\overline{50}$ YUL $=00$ ZUL $=5$

$X L R=50$ YLR $=00$ ZLR $=-185$

$U A X=1$ WDN $=-1$ NAX $=130 \quad L P I=8$ 
NCH=! UFCWMS! END PLOT

END DATA

END

- CSAS25

UF6_UO2F2_4_9843

27GR̄OUPNDF $\overline{4}$ INFHOMMEDIUM

UF6 11.03752939223597 .65922380 .8492234

1.5100

CARBONSTEEL 21.0

END

AL $30.05 .54-4$

CA $30.05 .30-3$

END

C $30.06 .59-3$

H 30.0 .0133

O $\quad 30.0 .0457$

FE $30.03 .59-4$

MG $30.03 .25-3$

SI $30.08 .43-3$

NA $30.03 .89-4$

S $30.01 .09-4$

$\mathrm{H} 2 \mathrm{O} 41.0$

NI 50.0 .0603

CU 50.0 .02649

FE 50.0 .00119

MN 50.0 .0006

END

END

END

END

END

END

END

END

END

END

END

END

END

END

END

SOLNUO2F2 632520 SPG $=4.71831 .0000293$

9223597.65922380 .84922341 .5100 END

END COMP

UF6 UO2F2 49843

READ PARAM $\overline{\mathrm{G}} \overline{\mathrm{D}} \mathrm{N}=203 \mathrm{END}$ PARAM

READ GEOM

CYLINDER $1110.16-136.9452-149.3928$

CYLINDER $6110.16 \quad-76.0553-149.3928$

CYLINDER $0110.16-24.9172-149.3928$

CYLINDER $5110.6273-24.45-149.86$

CYLINDER $0117.22440-149.86$

CYLINDER $2117.781 .27-150.495$

CUBOID $3250-5050-501.27-180$

END GEOM

READ BNDS XFC $=V A C$ YFC $=V A C$ END BNDS

READ PLOT TTL=!UF6 9843 !

$P L T=N O P I C=M I X T U R E$ XUL $=-50$ YUL $=00 \quad Z U L=5$

$X L R=50$ YLR $=00 Z L R=-185$

$U A X=1$ WDN $=-1 \quad N A X=130 \quad L P I=8$

$\mathrm{NCH}=$ ! UFCWMS! END PLOT

END DATA

END

UF6 $(H / U=8$, DRY RECEPT. $)$

= CSAS 25

UF6 UO2F2_8_2073

27GROOUPNDF $\overline{4}$ INFHOMMEDIUM

UF6 \ 1.03752939223597 .65922380 .8492234

1.5100

CARBONSTEEL 21.0

AL $30.05 .54-4$

CA $30.05 .30-3$

C $30.06 .59-3$

H 30.0 .0133

O 30.0 .0457

FE $30.03 .59-4$

MG $30.03 .25-3$

SI $30.08 .43-3$

END

END

END

END

END

END

END

END

END

END $\begin{array}{ll}\text { NA } 30.03 .89-4 & \text { END } \\ \mathrm{S} 30.01 .09-4 & \text { END } \\ \mathrm{H} 2 \mathrm{O} 41.0 & \text { END } \\ \mathrm{NI} 50.0 .0603 & \text { END } \\ \mathrm{CU} 50.0 .02649 & \text { END } \\ \text { FE } 50.0 .00119 & \text { END } \\ \text { MN } 50.0 .0006 & \text { END }\end{array}$

SOLNUO2F2 621700 SPG $=3.48131 .0000293$ 9223597.65922380 .84922341 .5100 END

END COMP

UF6 UO2F2 82073

READ PARAM $\bar{G}$ GN=203 END PARAM

READ GEOM

CYLINDER $1110.16-84.2655-149.3928$

CYLINDER $6110.16 \quad-75.0582-149.3928$

CYLINDER $0110.16-24.9172-149.3928$

CYLINDER 51 10.6273 -24.45 -149.86

CYLINDER $0117.22440-149.86$

CYLINDER $2117.781 .27-150.495$

CUBOID $3250-5050-50 \quad 1.27-180$

END GEOM

READ BNDS $X F C=V A C$ YFC $=V A C$ END BNDS

READ PLOT TTL=!UF6_UO2F2_4_2073!

$P L T=N O P I C=M I X T U R E \bar{E} X U L=-50$ YUL $=00$ ZUL $=5$

$X L R=50$ YLR $=00 \quad Z L R=-185$

$U A X=1$ WDN $=-1 \quad N A X=130 \quad L P I=8$

$\mathrm{NCH}=$ ! UFCWMS! END PLOT

END DATA

END

= CSAS25

UF6_UO2F2_8_2492

27GROUPNDF' INFHOMMEDIUM

UF6 11.03752939223597 .65922380 .8492234

1.5100

CARBONSTEEL 21.0

AL $30.05 .54-4$

CA $30.05 .30-3$

C $30.06 .59-3$

H 30.0 .0133

O 30.0 .0457

FE $30.03 .59-4$

MG $30.03 .25-3$

SI $30.08 .43-3$

NA $30 . C 3.89-4$

S $30.01 .09-4$

$\mathrm{H} 2 \mathrm{O} \quad 41.0$

NI 50.0 .0603

CU 50.0 .02649

FE 50.0 .00119

MN 50.0 .0006

END

END

END

END

END

END

END

END

END

END

END

END

END

END

END

END

SOLNUO2F2 621700 SPG $=3.48131 .0000293$

9223597.65922380 .84922341 .5100 END

END COMP

UF6_UO2F2_8_2492

READ PARAM $\bar{G} \bar{G} N=203$ END PARAM

READ GEOM

CYLINDER $1110.16-85.9102$-149.3928

CYLINDER $6110.16 \quad-74.3593-149.3928$

CYLINDER $0110.16-24.9172-149.3928$

CYLINDER $5110.6273-24.45-149.86$

CY!INDER $0117.22440-149.86$

CYLINDER $2117.781 .27-150.495$

CUBOID $3250-5050-50 \quad 1.27-180$ 
END GEOM

READ BNDS XFC=VAC YFC=VAC END BNDS

READ PLOT TTL=IUF6_UO2F2_4_2492!

PLT=NO PIC-MIXTURE XUL $=-50$ YUL $=00$ ZUL=5

$X L R=50$ YLR $=00$ ZLR=-185

$U A X=1$ WON $=-1 \quad N A X=130$ LPI $=8$

NCH=! UFCWMS! END PLOT

END DATA

END

$=$ CSAS25

UF6_UO2F2_8_3750

27GR̄OUPND̄F $\overline{4}$ INFHOMMEDIUM

UF6 11.03752939223597 .65922380 .8492234

\subsection{0}

CAREONSTEEL 21.0

END

AL $30.05 .54-4$

CA $30.05 .30-3$

C $30.06 .59-3$

H 30.0 .0133

O 30.0 .0457

FE $30.03 .59-4$

MG $30.03 .25-3$

SI $30.08 .43-3$

NA $30.03 .89-4$

END

END

END

END

END

END

END

END

END

S $30.01 .09-4$

$\mathrm{H} 2 \mathrm{O} 41.0$

NI 50.0 .0603

END

END

CU 50.0 .02649

END

END

END

MN 50.0 .0006

END

SOLNUO2F2 621700 SPG $=3.48131 .0000293$

9223597.65922380 .84922341 .5100 END

END COMP

UF6_UO2F2 8 -3750

READ PARAM GEN=203 END PARAM

READ GEOM

CYLINDER $1110.16-89.6445-149.3928$

CYLINDER 6110.16 -72.2624 -149.3928

CYLINDER $0110.16-24.9172-149.3928$

CYLINDER $5110.6273-24.45-149.86$

CYLINDER $0117.22440-149.86$

CYLINDER $2117.781 .27-150.495$

CUBOID $3250-5050-501.27-180$

END GEOM

READ BNDS XFC $=V A C$ YFC $=V A C E N D$ BNDS

READ PLOT TTL=IUF6 UO2F2 43750 !

$P L T=N O P I C=M I X T U R E \bar{E} X U L=-50 \quad \bar{Y} U L=00 \quad Z U L=5$

$X L R=50$ YLR $=00 \quad Z L R=-185$

$U A X=1$ WDN $=-1 \quad N A X=130 \mathrm{LPI}=8$

$\mathrm{NCH}=$ ! UFCWMS! END PLOT

END DATA

END

\section{- CSAS25}

UF6 UO2F2_8 7105

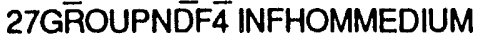

UF6 11.03752939223597 .65922380 .8492234

1.5100

CARBONSTEEL 21.0

AL $30.05 .54-4$

CA $30.05 .30-3$

C $30.06 .59-3$

H 30.0 .0133
END

END

END

END

END

END
O 30.00457

FE $30.03 .59-4$

MG $30.03 .25-3$

SI 30.08 .43 .3

NA $30.03 .89-4$

S $30.01 .09-4$

$\mathrm{H} 2 \mathrm{O} 41.0$

NI 50.0 .0603

CU 50.0 .02649

FE 50.0 .00119

MN 50.0 .0006

END

END

END

END

END

END

END

END

END

END

END

SOLNUO2F2 621700 SPG-3.48131.0000 293

92235 97.65.92238 0.8492234 1.5100 END

END COMP

UF6 UO2F2 87105

READ PARAM $\bar{G} E N=203$ END PARAM

READ GEOM

CYLINDER $1110.16-99.6026-149.3928$

CYLINDER $6110.16-66.6706-149.3928$

CYLINDER $0110.16-24.9172-149.3928$

CYLINDER $5110.6273-24.45-149.86$

CYLINDER $0117.22440-149.86$

CYLINDER $2117.781 .27-150.495$

CUBOID $3250-5050-501.27-180$

END GEOM

READ BNDS $X F C=V A C$ YFC $=V A C$ END BNDS

READ PLOT TTL=IUF6 UO2F2 47105 !

$P L T=N O P I C=M I X T U R E$ XUL $=-50$ YUL $=00 \quad Z U L=5$

$X L R=50$ YLR $=00 \quad Z L R=; 185$

$U A X=1$ WDN $=-1$ NAX $=130$ LPI $=8$

$N C H=$ I UFCWMS! END PLOT

END DATA

END

= CSAS25

UF6 UO2F2 $8 \quad 11299$

$27 \mathrm{G} \overline{\mathrm{A}}$ OUPNDF $\overline{4}$ INFHOMMEDIUM

UF6 11.03752939223597 .65922380 .8492234

1.5100

CARBONSTEEL 21.0

END

$\mathrm{AL} \quad 30.05 .54-4$

CA $30.05 .30-3$

C $30.06 .59-3$

H 30.0 .0133

O 30.0 .0457

FE $30.03 .59-4$

MG $30.03 .25-3$

SI $30.08 .43-3$

NA $30.03 .89-4$

S $30.01 .09-4$

$\mathrm{H} 2 \mathrm{O} 41.0$

NI 50.0 .0603

CU 50.0 .02649

FE 50.0 .00119

MN 50.0 .0006

END

END

END

END

END

END

END

END

END

END

END

END

END

END

END

END

SOLNUO2F2 621700 SPG $=3.48131 .0000293$ 9223597.65922380 .84922341 .5100 END

END COMP

UF6 UO2F2 8 11299

READ PARAM $\bar{G}$ EN $=203$ END PARAM

READ GEOM

CYLINDER $1110.16-112.0501-149.3928$

CYLINDER $6110.16-59.6810-149.3928$

CYLINDER $0110.16-24.9172-149.3928$ 
CYLINDER $5110.6273-24.45-149.86$

CYLINDER $0117.22440-149.86$

CYLINDER $2117.781 .27-150.495$

CUBOID $3250-5050-501.27-180$

END GEOM

READ BNDS XFC $=V A C$ YFC $=V A C$ END BNDS

READ PLOT TTL=!UF6 UO2F2 4 11299!

$P L T=N O P I C=M I X T U R E \overline{X U L}=-50$ YUL $=00$ ZUL $=5$

$X L R=50$ YLR $=00$ ZLR $=-185$

$U A X=1$ WDN $=-1 \quad N A X=130$ LPI $=8$

$\mathrm{NCH}=$ ! UFCWMS! END PLOT

END DATA

END

\section{$=$ CSAS25}

UF6_UO2F2 8 15492

27GROUPNDF $\overline{4}$ INFHOMMEDIUM

UF6 11.03752939223597 .65922380 .8492234 1.5100

CARBONSTEEL 21.0

END

AL $30.05 .54-4$

CA $30.05 .30-3$

C $30.06 .59-3$

H 30.0 .0133

O $\quad 30.0 .0457$

FE $30.03 .59-4$

MG $30.03 .25-3$

SI $30.08 .43-3$

NA $30.03 .89-4$

S $30.01 .09-4$

$\mathrm{H} 2 \mathrm{O} \quad 41.0$

NI 50.0 .0603

CU 50.0 .02649

FE 5 0.0.00119

MN 50.0 .0006

END

END

END

END

END

END

END

END

END

END

END

END

END

END

END

SOLNUO2F2 621700 SPG $=3.48131 .0000293$ 9223597.65922380 .84922341 .5100 END

END COMP

UF6 UO2F2 8 - 15492

READ PARAM $\overline{\mathrm{G}} \overline{\mathrm{D}} \mathrm{N}=203$ END PARAM

READ GEOM

CYLINDER $1110.16-124.4977-149.3928$

CYLINDER $6110.16-52.6913-149.3928$

CYLINDER $0110.16-24.9172-149.3928$

CYLINDER $5110.6273-24.45-149.86$

CYLINDER $0117.22440-149.86$

CYLINDER $2117.781 .27-150.495$

CUBOID $3250-5050-501.27-180$

END GEOM

READ BNDS XFC=VAC YFC=VAC END BNDS

READ PLOT TTL=!UF6 UO2F2 $4 \quad 15492$ !

$P L T=N O P I C=M I X T U R E$ XUL $=-50$ Y $U L=00 Z U L=5$

$X L R=50$ YLR $=00 Z L R=-185$

$U A X=1$ WDN $=-1 \quad N A X=130 L P I=8$

$\mathrm{NCH}=$ ! UFCWMS! END PLOT

END DATA

END

= CSAS25

UF6 UO2F2 $8 \quad 19686$

27GRिOUPNDF $\overline{4}$ INFHOMMEDIUM

UF6 11.03752939223597 .65922380 .8492234

1.5100

END

CARBONSTEEL 21.0
AL $\quad 30.05 .54-4$

END

CA $30.05 .30-3$

C $30.06 .59-3$

H 30.0 .0133

O 30.0 .0457

FE $30.03 .59-4$

MG $30.03 .25-3$

SI $30.08 .43-3$

NA $30.03 .89-4$

S $30.01 .09-4$

$\mathrm{H} 2 \mathrm{O} \quad 41.0$

NI 50.0 .0603

CU 50.0 .02649

FE 50.0 .00119

MN 50.0 .0006

SOLNUO2F2 621700 SPG $=3.48131 .0000293$

9223597.65922380 .84922341 .5100 END

END COMP

UF6 UO2F2 $8 \quad 19686$

READ PARA $\bar{M} \bar{G} \overline{\mathrm{G}}=203$ END PARAM

READ GEOM

CYLINDER $1110.16-136.9452-149.3928$

CYLINDER $6110.16-45.7016-149.3928$

CYLINDER $0110.16-24.9172-149.3928$

CYLINDER $5110.6273-24.45-149.86$

CYLINDER $0117.22440-149.86$

CYLINDER $2117.781 .27-150.495$

CUBOID $3250-5050-501.27-180$

END GEOM

READ BNDS XFC $=V A C$ YFC $=V A C$ END BNDS

READ PLOT TTL=!UF6 19686!

$P L T=N O P I C=M I X T U R E$ XUL $=-50 \quad Y U L=00 Z U L=5$

$X L R=50 \quad Y L R=00 Z L R=-185$

$U A X=1$ WDN $=-1 \quad N A X=130 \mathrm{LPI}=8$

$\mathrm{NCH}=$ ! UFCWMS! END PLOT

END DATA

END

\section{OXIDES IN 6-INCH CANISTERS}

=CSAS25

U $308155 \mathrm{~W} \mathrm{D}$

27GROUPNDF4 INFHOMMEDIUM

U-234 $10.05 .4837 \mathrm{E}-05$

U-235 $10.03 .5527 E-03$

U-238 $10.03 .0524 E-05$

H $10.05 .4571 \mathrm{E}-02$

O 10.0 3.6987E-02

CARBONSTEEL 21.0

AL 3 0.0 5.54-4

CA $30.05 .30-3$

C $30.06 .59-3$

H 30.0 .0133

O 30.0 .0457

FE $30.03 .59-4$

MG $30.03 .25-3$

SI $30.08 .43-3$

NA $30.03 .89-4$

S $30.01 .09-4$

$\mathrm{H} 2 \mathrm{O} 41.0$

END COMP

U3O8 $155 \mathrm{~W}$ D

READPARAM $\bar{R} U N=$ YES END PARAM

READ GEOM 
CYLINDER $117.62 .718221-19.05$

CYLINDER $017.6219 .05-19.05$

CYLINDER 217.699 19.129-19.129

CYLINDER $4110.9537548 .181-19.129$

CYLINDER $2111.2458549 .451-19.764$

CUBOID $3350 \quad-5050-50 \quad 49.451-50.549$

END GEOM

READ BNDS XFC $=V A C$ YFC $=V A C$ END BNDS

READ PLOT TTL $=1 U 308155 \mathrm{~W}$ D!

PLT $=$ YES PIC $=M I X T U R E X U \bar{L}=-50$ YUL $=00$

$Z U L=50 X L R=50$ YLR $=00 Z Z R=-30$

$U A X=1$ WDN $=-1$ NAX $=130 \mathrm{LPI}=8$

$\mathrm{NCH}=$ ! UFCW ! END PLOT

END DATA

END

$=$ C.SAS25

UO2 $105 W$ D

27GR̄OUPNDDF4 INFHOMMEDIUM

U-234 $10.08 .1100-5$

U.235 $10.05 .254-3$

U-238 $10.04 .51-5$

H $10.05 .3804-2$

O $10.03 .7663-2$

CARBONSTEEL 21.0

AL $30.05 .54-4$

CA $30.05 .30-3$

C $30.06 .59-3$

H $30 . C .0133$

030.0 .0457

FE $30.03 .59-4$

MG $30.03 .25-3$

SI $30.08 .43-3$

NA $30.03 .89-4$

S $30.01 .09-4$

END

END

END

END

END

END

END

END

END

END

END

END

END

END

END

END

$\mathrm{H} 2 \mathrm{O} 41.0$

END COMP

UO2 105 W D

READ PARAM $\bar{R} U N=$ YES END PARAM

READ GEOM

CYLINDER $117.62-5.68347-19.05$

CYLINDER $017.6219 .05-19.05$

CYLINDER $217.69919 .129-19.129$

CYLINDER $4110.9537548 .181-19.129$

CYLINDER $2111.2458549 .451-19.764$

CUBOID $3350-5050-5049.451-50.549$

END GEOM

READ BNDS XFC $=V A C$ YFC $=V A C$ END BNDS

READ PLOT TTL=IUO2_10_5_W_D!

$P L T=Y E S \quad P I C=M I X T U R E X U L=-50 \quad Y U L=00$

$Z U L=50 X L R=50 Y L R=00 Z L R=-30$

$U A X=1$ WDN $=-1 \quad N A X=130 \mathrm{LPI}=8$

$\mathrm{NCH}=$ !UFCW $\mid$ END PLOT

END DATA

END

$=$ CSAS 25

UO2 10 10.5 W D

27GR̄OUPNDF4 INFHOMMEDIUM

U-234 $10.08 .1100-5$

U-235 $10.05 .254-3$

Eir?

U-238 $10.04 .51-5$

H $10.05 .3804-2$

$010.03 .7663-2$ $\begin{array}{ll}\text { CARBONSTEEL 2 1.0 } & \text { END } \\ \text { AL } 30.05 .54-4 & \text { END } \\ \text { CA } 30.05 .30-3 & \text { END } \\ \text { C } 30.06 .59-3 & \text { END } \\ \text { H } 30.0 .0133 & \text { END } \\ \text { O } 30.0 .0457 & \text { END } \\ \text { FE } 30.03 .59-4 & \text { END } \\ \text { MG } 30.03 .25-3 & \text { END } \\ \text { SI } 30.08 .43-3 & \text { END } \\ \text { NA } 30.03 .89-4 & \text { END } \\ \text { S } 30.01 .09-4 & \text { END } \\ \text { H2O } 41.0 & \text { END }\end{array}$

END COMP

UO2_10_10.5_W_D

READ PATRAM $R \bar{U} \bar{N}=Y E S$ END PARAM

READ GEOM

CYLINDER $117.629 .01972-19.05$

CYLINDER $017.6219 .05-19.05$

CYLINDER $217.69919 .129-19.129$

CYLINDER $4110.9537548 .181-19.129$

CYLINDER $2111.2458549 .451-19.764$

CUBOID $3350-5050-5049.451-50.549$

END GEOM

READ BNDS XFC $=V A C$ YFC $=V A C$ END BNDS

READ PLOT TTL $=$ !UO2 10_10.5 W D!

$P L T=Y E S P I C=M I X T U R E X \bar{U} L=-50$ YUL $=00$

$Z U L=50 X L R=50$ YLR $=00$ ZLR $=-30$

$U A X=1 W D N=-1 \quad N A X=130 \quad L P \mid=8$

$\mathrm{NCH}=$ ! UFCW ! END PLOT

END DATA

END

$=$ CSAS25

UO2_10_8_D_D

27GR̄OUPN̄DF4 INFHOMMEDIUM

U-234 $10.08 .1100-5$

U-235 $10.05 .254-3$

U-238 $10.04 .51-5$

H $10.05 .3804-2$

O $10.03 .7663-2$

CARBONSTEEL 21.0

AL 3 0.0 5.54-4

CA $30.05 .30-3$

C $30.06 .59-3$

H3 0.0 .0133

O 30.0 .0457

FE $30.03 .59-4$

MG $30.03 .25-3$

SI $30.08 .43-3$

NA $30.03 .89-4$

S $30.01 .09-4$

$\mathrm{H} 2 \mathrm{O} 41.0$

END COMP

UO2 $108 \mathrm{D} D$

READ PATAMM RUN=YES END PARAM

READ GEOM

CYLINDER $117.622 .3364508-19.05$

CYLINDER $017.6219 .05-19.05$

CYLINDER $217.69919 .129-19.129$

CYLINDER $0110.9537548 .181-19.129$

CYLINDER $2111.2458549 .451-19.764$

CUBOID $3350-5050-5049.451-50.549$

END GEOM

READ BNDS XFC $=$ VAC YFC $=$ VAC END BNDS 
READ PLOT TTL=!UO2_10_8_D_D!

PLT $=$ YES PIC $=M I X T U R E$ XUL $=-\overline{50}$ YUL $=00$

$Z U L=50$ XLR $=50$ YLR $=00$ ZLR $=-30$

$U A X=1$ WDN $=-1 \quad N A X=130 L P l_{2}=8$

$\mathrm{NCH}=$ = UFCW IEND PLOT

END DATA

END

-CSAS25

UO2 108 D D REG

27GR̄OUPNDDF4 INFHOMMEDIUM

U-234 $10.08 .1100-5$

U-235 $10.05 .254-3$

END

U-238 $10.04 .51-5$

END

H $10.05 .3804-2$

$010.03 .7663-2$

CARBONSTEEL 21.0

END

END

END

$\begin{array}{ll}\text { REG-CONCRETE } 31 & \text { END } \\ \mathrm{H} 2 \mathrm{O} 41.0 & \text { END }\end{array}$

END COMP

UO2 10_8_D_D_REG

REA $\bar{D}$ PARAM $\vec{A} \bar{U}=$ Y $=$ ES END PARAM

READ GEOM

CYLINDER 117.622 .3364508 -19.05

CYLINDER $017.6219 .05-19.05$

CYLINDER $217.69919 .129-19.129$

CYLINDER $0110.9537548 .181-19.129$

CYLINDER $2111.2458549 .451-19.764$

CUBOID $3350-5050-5049.451-50.549$

END GEOM

READ BNDS XFC $=V A C$ YFC $=V A C$ END BNDS

READ PLOT TTL=!UO2_10_8_D_D_REG!

$P L T=Y E S P I C=M I X T U R E \bar{U}$ X $=-50$ YUL $=00$

$Z U L=50 \quad X L R=50$ YLR $=00 \quad Z L R=-30$

$U A X=1 W D N=-1 \quad N A X=130 L P I=8$

$\mathrm{NCH}=$ !UFCW $\mid$ END PLOT

END DATA

END

\section{$=$ CSAS 25}

UO2 10 8 D D INF

27GR̄OUPND $\overline{\text { F } 4} 4$ INFHOMMEDIUM

U-234 $10.08 .1100-5$

U-235 $10.05 .254-3$

U-238 $10.04 .51-5$

END

H $10.05 .3804-2$

O $10.03 .7663-2$

CARBONSTEEL 21.0

AL $30.05 .54-4$

CA $30.05 .30-3$

C $30.06 .59-3$

H 30.0 .0133

O 30.0 .0457

FE $30.03 .59-4$

MG $30.03 .25-3$

SI $30.08 .43-3$

NA $30.03 .89-4$

S $30.01 .09-4$

$\mathrm{H} 2 \mathrm{O} 41.0$

END COMP

UO2_10_8D D INF

READ GE $\bar{E} \overline{\mathrm{M}}$

UNIT 1

ZHEMICYL+X $117.622 .3364508-19.05$
ZHEMICYL+X $017.622 P 19.05$

ZHFMICYL+X $217.6992 \mathrm{P} 19.129$

ZHEMICYL+X $0110.9537548 .181-19.129$

ZHEMICYL+X 2111.2458549 .451 -19.764

CUBOID $31105.5675030 .48-30.4849 .451$

$-35.004$

HOLE 2105.567500

HOLE 352.7837530 .480

HOLE $452.78375-30.480$

UNIT 2

ZHEMICYL-X 117.622 .3364508 -19.05

ZHEMICYL-X 017.62 2P19.05

ZHEMICYL-X 217.699 2P19.129

ZHEMICYL-X $0110.9537548 .181-19.129$

ZHEMICYL-X $2111.2458549 .451-19.764$

UNIT 3

ZHEMICYL-Y 117.622 .3364508 -19.05

ZHEMICYL-Y 017.622 2P19.05

ZHEMICYL-Y 217.6992 2P19.129

ZHEMICYL-Y 0110.9537548 .181 -19.129

ZHEMICYL-Y 2111.2458549 .451 -19.764 UNIT 4

ZHEMICYL+Y $117.622 .3364508-19.05$

ZHEMICYL+Y $017.622 P 19.05$

ZHEMICYL+Y $217.6992 P 19.129$

ZHEMICYL+Y $0110.9537548 .181-19.129$

ZHEMICYL+Y $2111.2458549 .451-19.764$

END GEOM

READ BNDS XFC=SPEC YFC $=S P E C$ END BNDS

READ PLOT TTL=!UO2_10_8_D_D_INF!

$P L T=Y E S P I C=M I X T U R \bar{E} X \bar{U} L=0 \bar{Y} \bar{U}=35 Z Z L=0$

$X L R=110 \quad Y L R=-35 \quad Z L R=0$

$U A X=1$ VDN $=-1 \quad N A X=130 \quad L P \mid=8$

$\mathrm{NCH}=$ ! UF.W IEND PLOT

END DATA

END

=CSAS25

UO2 108 M M 0.5

27GR̄OUPNDDF4 INFHOMMEDIUM

$\mathrm{U}-23410.08 .1100-5$

U-235 $10.05 .254-3$

U-2381 $0.04 .51-5$

H $10.05 .3804-2$

O $10.03 .7663-2$

CARBONSTEEL 21.0

AL $\quad 30.05 .54-4$

CA $30.05 .30-3$

C $30.06 .59-3$

H 30.0 .0133

O 30.0 .0457

FE $30.03 .59-4$

MG $30.03 .25-3$

SI $30.08 .43-3$

NA $30.03 .89-4$

S $30.01 .09-4$

$U-23440.0 \quad 3.4585 E-07 \quad$ END

U-235 $40.02 .2407 E-05 \quad$ END

U-23840.0 1.9251E-07 END

H $40.06 .6804 E-02$ END

O $40.03 .3448 E-02$ END

END COMP

UO2 108 M M 0.5

READ PARAM $\bar{R} \bar{U} N=Y E S$ END PARAM 
READ GEOM

CYLINDER $117.62 \quad 1.83645-19.05$

CYLINDER $417.6219 .05-19.05$

CYLINDER $217.69919 .129-19.129$

CYLINDER $4110.9537548 .181-19.129$

CYLINDER $2111.2458549 .451-19.764$

CUBOID $3350-5050-5049.451-50.549$

END GEOM

READ BNDS XFC=VAC YFC=VAC END BNDS

READ PLOT TTL $=\mid U O 2$ 2 10_8_M_M_0.5 !

$P L T=Y E S$ PIC $=M I X T U R E X \bar{U}=-50$ YUL $=00$

$Z U L=50$ XLR $=50$ YLR $=00$ ZLR $=-30$

$U A X=1$ WDN $=-1 \quad N A X=130 \quad L P I=8$

$\mathrm{NCH}=$ ! UFCWIEND PLOT

END DATA

END

$=$ CSAS25

UO2 $108 \mathrm{M} \mathrm{M} 1$

27GROUPND $\bar{F} 4$ INFHOMMEDIUM

U-234 $10.08 .1100-5 \quad$ END

U.235 $10.05 .254-3 \quad$ END

U-238 $10.04 .51-5 \quad$ END

H $10.05 .3804-2 \quad$ END

O $10.03 .7663-2 \quad$ END

CARBONSTEEL $21.0 \quad$ END

AL $30.05 .54-4 \quad$ END

CA $30.05 .30-3 \quad$ END

C $30.06 .59-3$ END

H $30.0 .0133 \quad$ END

O 30.0 .0457 END

FE 3 $0.03 .59-4 \quad$ END

MG $30.03 .25-3$ END

SI $30.08 .43-3 \quad$ END

NA $30.03 .89-4$ END

$S$ S $30.01 .09-4 \quad$ END

U-234 $40.06 .8877 E-07 \quad$ END

U-235 $40.04 .4623 E-05 \quad$ END

U-238 $40.03 .8339 E-07$ END

H $40.06 .6749 \mathrm{E}-02$ END

O $40.03 .3466 \mathrm{E}-02$ END

END COMP

UO2 10 8 M M 1

READ PATRAM RŪN = YES END PARAM

READ GEOM

CYLINDER $117.62 \quad 1.33645$-19.05

CYLINDER $417.6219 .05-19.05$

CYLINDER $217.69919 .129-19.129$

CYLINDER $4110.9537548 .181-19.129$

CYLINDER $2111.2458549 .451-19.764$

CUBOID $3350-5050-5049.451-50.549$

END GEOM

READ BNDS XFC $=V A C$ YFC $=V A C$ END BNDS

READ PLOT TTL=!UO2 $108 \mathrm{M} \mathrm{M} 1$ !

PLT $=Y E S$ PIC $=$ MIXTURE XUL $=-50$ YUL $=00$

$Z U L=50 \quad X L R=50$ YLR $=00$ ZLR $=-30$

$U A X=1$ WDN $=-1 \quad N A X=130 \quad L P I=8$

$N C H=$ ! UFCW!END PLOT

END DATA

END

=CSAS25

UO2 $108 \mathrm{M} \mathrm{M} 2$

27GR̄OUPNDTF4 INFHOMMEDIUM
U-234 $10.08 .1100-5$

END

U-235 $10.05 .254-3$

U-2381 $0.04 .51-5$

H $10.05 .3804-2$

O $10.03 .7663-2$

CARBONSTEEL 21.0

AL $30.05 .54-4$

CA $30.05 .30-3$

C $30.06 .59-3$

H 30.0 .0133

O $\quad 30.0 .0457$

FE $\quad 30.03 .59-4$

MG $30.03 .25-3$

SI $30.08 .43-3$

NA $30.03 .89-4$

S $30.01 .09-4$

U-234 40.0 1.3659E-06

U-235 40.0 8.8495E-05

U-238 40.0 7.6033E-07

H $40.0 \quad 6.6640 \mathrm{E}-02$

O 40.0 3.3501E-02

END COMP

UO2 $108 \mathrm{M} \mathrm{M} 2$

READ PARAM $\bar{R} \bar{U} \bar{N}=$ YES END PARAM

READ GEOM

CYLINDER $117.62 \quad 0.33645-19.05$

CYLINDER $417.6219 .05-19.05$

CYLINDER $217.69919 .129-19.129$

CYLINDER $4110.9537548 .181-19.129$

CYLINDER $2111.2458549 .451-19.764$

CUBOID $3350-5050-5049.451-50.549$

END GEOM

READ BNDS $X F C=V A C$ YFC $=V A C$ END BNDS

READ PLOT TTL =!UO2_10_8_M_M_2!

$P L T=Y E S$ PIC $=$ MIXTURE XUL $=-50$ YUL $=00$

$Z U L=50$ XLR $=50$ YLR $=00$ ZLR $=-30$

$U A X=1$ WDN $=-1 \quad N A X=\left.130 \quad L P\right|_{=8}$

$\mathrm{NCH}=$ ! UFCW!END PLOT

END DATA

END

\section{=CSAS25}

UO2_10_8_M_M_4

27GROUPNDFA INFHOMMEDIUM

U-234 $10.08 .1100-5$

U-235 $10.05 .254-3$

U.238 $10.04 .51-5$

H $10.05 .3804-2$

O $10.03 .7663-2$

CARBONSTEEL 21.0

AL $30.05 .54-4$

CA $30.05 .30-3$

C $30.06 .59-3$

H 30.0 .0133

O 30.0 .0457

FE $30.03 .59-4$

MG $30.03 .25-3$

SI $30.08 .43-3$

NA $30.03 .89-4$

S $30.01 .09-4$

U-234 40.0 2.6866E-06

U-235 40.01 .7406 E-04

U-238 40.0 1.4955E-06

H $40.06 .6427 E-02$

END

END

END

END

END

END

END

END

END

END

END

END

END

END

END

END

END

END

END

END 
O $40.03 .3570 E-02$

END COMP

UO2 $108 \mathrm{M} \mathrm{M} 4$

READ PATRAM RŪ $=$ YES END PARAM

READ GEOM

CYLINDER 117.62 -1.66355 - 19.05

CYLINDER $417.6219 .05-19.05$

CYLINDER 217.699 19.129-19.129

CYLINDER $4110.9537548 .181-19.129$

CYLINDER $2111.2458549 .451-19.764$

CUBOID $3350-5050-50 \quad 49.451-50.549$

END GEOM

READ BNDS XFC $=V A C$ YFC $=V A C$ END BNDS

READ PLOT TTL=IUO2 $108 \mathrm{M} \mathrm{M} 4 !$

$P L T=Y E S P I C=M I X T U R \bar{E} X \overline{U L}=-50 \overline{Y U L}=00$

$Z U L=50 X L R=50$ YLR $=00 Z L R=-30$

$U A X=1$ WDN $=-1 \quad N A X=130 \quad L P I=8$

$\mathrm{NCH}=$ ! UFCW! END PLOT

END DATA

END

\section{$=$ CSAS25}

UO2 108 8 M M 6

27GR̄OUPN̄DFA INFHOMMEDIUM

U-234 $10.08 .1100-5$

U-235 $10.05 .254-3$

END

U-238 $10.04 .51-5$

H $10.05 .3804-2$

O $10.03 .7663-2$

CARBONSTEEL 21.0

END

END

END

END

AL $30.05 .54-4$

END

CA $30.05 .30-3$

END

C $30.06 .59-3$

END

H 30.0 .0133

O $\quad 30.0 .0457$

FE $30.03 .59-4$

MG $30.03 .25-3$

SI $30.08 .43-3$

NA $30.03 .89-4$

S $30.01 .09-4$

U.234 $40.03 .9643 E-06$

U-235 40.0 2.5683E-04

U-238 40.02 .2066 E-06

H $40.06 .6222 \mathrm{E}-02$

O $40.03 .3637 E-02$

END COMP

UO2 $108 \mathrm{MM} 6$

READ PARAMM RUN $=$ YES END PARAM

READ GEOM

CYLINDER $117.62 \quad-3.66355-19.05$

CYLINDER $417.6219 .05-19.05$

CYLINDER 217.699 19.129 -19.129

CYLINDER $4110.9537548 .181-19.129$

CYLINDER $2111.2458549 .451-19.764$

CUBOID $3350-50 \quad 50-5049.451-50.549$

END GEOM

READ BNDS XFC=VAC YFC $=V A C$ END BNDS

READ PLOT TTL=!UO2 $10 \_8 \_M \_M \_6 !$

PLT $=$ YES PIC $=$ MIXTURE XUL $=-50$ YUL $=00$

$Z U L=50 \quad X L R=50$ YLR $=00 \quad Z L R=-30$

$U A X=1 W D N=-1 \quad N A X=130 \mathrm{LPI}=8$

$\mathrm{NCH}=$ ! UFCW!END PLOT

END DATA

END

\section{= CSAS25}

UO2 10 8 M M 8

27GR̄OUPN NDF 4 INFHOMMEDIUM

U-234 $10.08 .1100-5$

U-235 $10.05 .254-3$

U-2381 $0.04 .51-5$

H $10.05 .3804-2$

O $10.03 .7663-2$

CARBONSTEEL 21.0

$\mathrm{AL} \quad 30.05 .54-4$

CA $30.05 .30-3$

C $30.06 .59-3$

H 30.0 .0133

O 30.0 .0457

FE $30.03 .59-4$

MG $30.03 .25-3$

SI $30.08 .43-3$

NA $30.03 .89-4$

S 30.01 .09 .4

U-234 40.0 5.2009E-06

U-235 40.0 3.3695E-04

U-238 40.0 2.8950E-06

H $40.06 .6023 E-02$

O 40.0 3.3701E-02

END COMP

UO2 $108 \mathrm{M} \mathrm{M} 8$

READ PARAM RUN $=$ YES END PARAM

READ GEOM

CYLINDER $117.62 \quad-5.66355-19.05$

CYLINDER $417.6219 .05-19.05$

CYLINDER $217.69919 .129-19.129$

CYLINDER $4110.9537548 .181-19.129$

CYLINDER $2111.2458549 .451-19.764$

CUBOID $3350-5050-5049.451-50.549$

END GEOM

READ BNDS XFC=VAC YFC $=V A C$ END BNDS

READ PLOT TTL $=$ !UO2 $108 \mathrm{M} \mathrm{M} 8$ !

PLT $=$ YES PIC $=$ MIXTURE XUL $=-50$ YUL $=00$

$Z L L=50 X L R=50$ YLR $=00$ ZLR $=-30$

$U A X=1$ WDN $=-1 \quad N A X=130 \quad L P \mid=8$

$\mathrm{NCH}=$ ! UFCW!END PLOT

END DATA

END

=CSAS 25

UO2 108 M M 10

27GR̄OUPNDFF 4 INFHOMMEDIUM

U-2341 $0.08 .1100-5$

U-235 $10.05 .254-3$

U-238 1 0.0 4.51-5

H $10.05 .3804-2$

O 10.037663 .2

CARBONSTEEL 21.0

AL $30.05 .54-4$

CA $30.05 .30-3$

C $30.06 .59-3$

H 30.0 .0133

O 30.0 .0457

FE $30.03 .59-4$

MG $30.03 .25-3$

SI $30.08 .43-3$

NA $30.03 .89-4$

S $30.01 .09-4$
END

END

END

END

END

END

END

END

END

END

END

END

END

END

END

END 


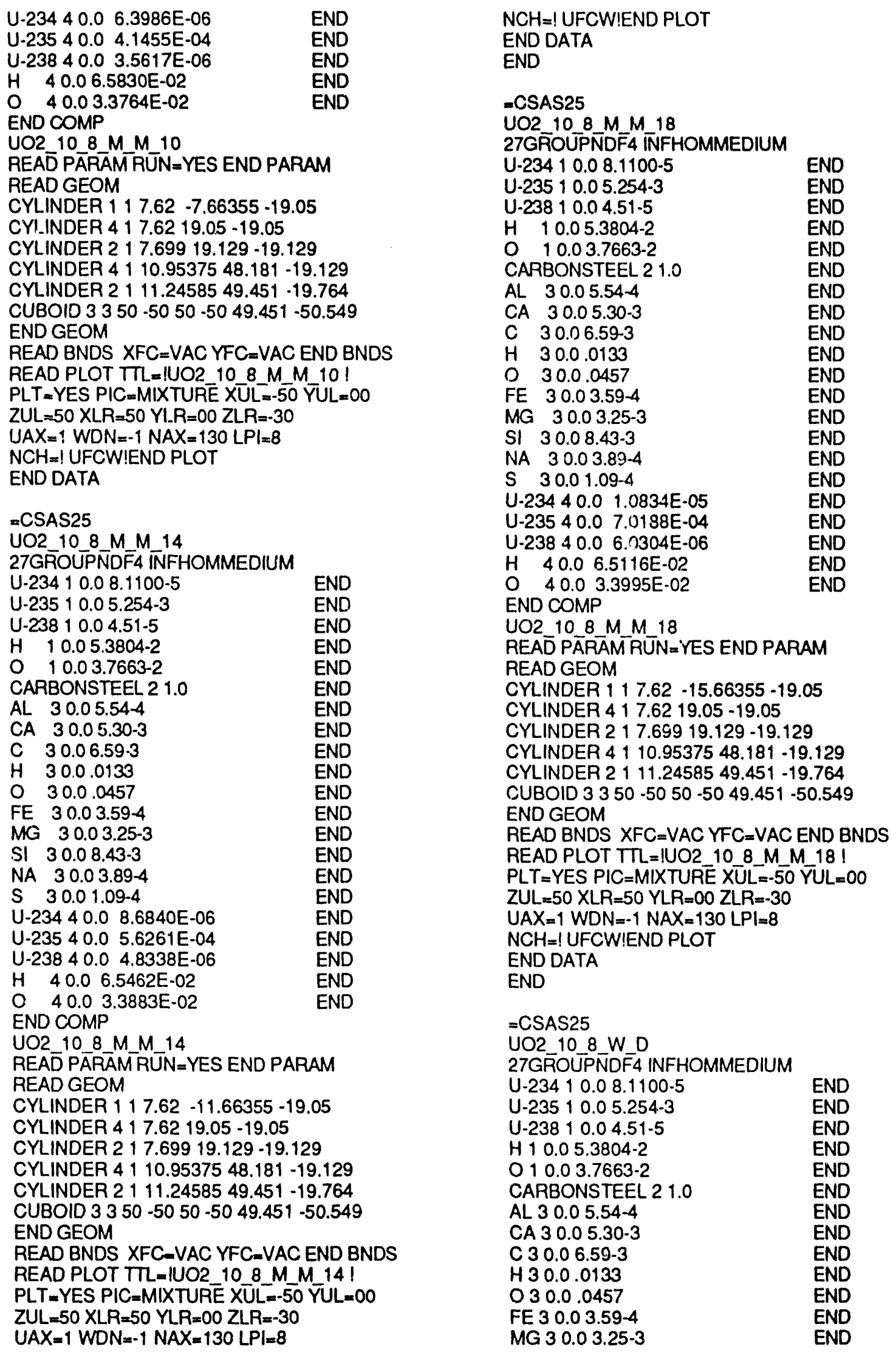




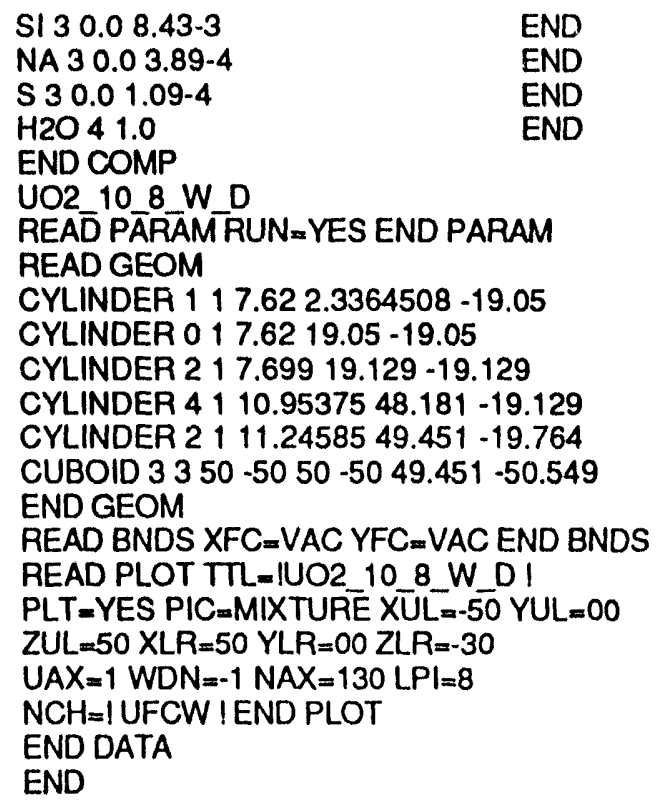

-CSAS25

UO2 108 W D H2O

27GR̄OUPNDDF4 INFHOMMEDIUM

U-234 $10.08 .1100-5$

U-235 $10.05 .254-3$

END

U-238 $10.04 .51-5$

END

H $10.05 .3804-2$

O $10.03 .7663-2$

CARBONSTEEL 21.0

REG-CONCRETE 31.0

END

END

END

END

$\mathrm{H} 2 \mathrm{O} 41.0$

END COMP

END

UO2 $108 W$ D H2O

READ PARAM $\bar{R} \overline{\mathrm{N}}=$ YES END PARAM

READ GEOM

CYLINDER $117.622 .3364508-19.05$

CYLINDER $017.6219 .05-19.05$

CYLINDER $217.69919 .129-19.129$

CUBOID 4328 -28 28 -28 39 -39

END GEOM

READ BNDS XFC $=V A C$ YFC $=V A C$ END BNDS

READ PLOT TTL=IUO2 10 8_W_D_H2O!

$P L T=Y E S$ PIC $=M I X T U R E \bar{U} \bar{U}=-50$ YUL $=00$

$Z U L=50 X L R=50$ YLR $=00$ ZLR $=-50$

$U A X=1$ WDN $=-1 \quad N A X=130 \quad L P \mid=8$

$\mathrm{NCH}=$ ! UFCW ! END PLOT

END DATA

END

$=$ CSAS 25

UO2 $108 \mathrm{~W}$ D INF

27GR̄OUPNDFA INFHOMMEDIUM

U-234 $10.08 .1100-5$

END

U-235 $10.05 .254-3$

END

U-238 $10.04 .51-5$

END

H $10.05 .3804-2$

END

O $10.03 .7663-2$

END

CARBONSTEEL 21.0 END

AL 3 0.0 5.54-4

END

CA $30.05 .30-3$

END

$\begin{array}{ll}\text { C } 30.06 .59-3 & \text { END } \\ \text { H } 30.0 .0133 & \text { END } \\ \text { O } 30.0 .0457 & \text { END } \\ \text { FE } 30.03 .59-4 & \text { END } \\ \text { MG } 30.03 .25-3 & \text { END } \\ \text { SI } 30.08 .43-3 & \text { END } \\ \text { NA } 30.03 .89-4 & \text { END } \\ \text { S } 30.01 .09-4 & \text { END } \\ \text { H2O } 41.0 & \text { END }\end{array}$

H2O 41.0

UO2 10_8_W_D_INF

READ GE $\bar{B} \bar{M}$

UNIT 1

ZHEMICYL +X $117.622 .3364508-19.05$

ZHEMICYL+X $017.622 P 19.05$

ZHEMICYL+X 217.699 2P19.129

ZHEMICYL+X $4110.9537548 .181-19.129$

ZHEMICYL+X $2111.2458549 .451-19.764$

CUBOID $31105.5675030 .48-30.4849 .451$

$-35.004$

HOLE 2105.567500

HOLE 352.7837530 .480

HOLE $452.78375-30.480$

UNIT 2

ZHEMICYL-X 117.622 .3364508 -19.05

ZHEMICYL-X 017.62 2P19.05

ZHEMICYL-X 217.699 2P19.129

ZHEMICYL-X $4110.9537548 .181-19.129$

ZHEMICYL-X $2111.2458549 .451-19.764$

UNIT 3

ZHEMICYL-Y 117.622 .3364508 -19.05

ZHEMICYL-Y 017.62 2P19.05

ZHEMICYL-Y 217.6992 2P19.129

ZHEMICYL-Y 4110.9537548 .181 -19.129

ZHEMICYL-Y 2111.2458549 .451 -19.764

UNIT 4

ZHEMICYL+Y 117.622 .3364508 -19.05

ZHEMICYL+Y 017.62 2P19.05

ZHEMICYL+Y $217.6992 P 19.129$

ZHEMICYL+Y $4110.9537548 .181-19.129$

ZHEMICYL+Y $2111.2458549 .451-19.764$

END GEOM

READ BNDS XFC=SPEC YFC $=$ SPEC END BNDS

READ PLOT TTL $=\mid U O 2$ 2 $108 \mathrm{~W} D$ D INFI

$P L T=Y E S$ PIC $=M I X T U R \bar{E} X \bar{U} L=0 \bar{Y} U \bar{L}=35 \quad Z U L=0$

$X L R=110$ YLR $=-35 \quad Z L R=0$

$U A X=1$ VDN $=-1 \quad N A X=130 \quad L P I=8$

$\mathrm{NCH}=$ ! UF.W I END PLOT

END DATA

END

$=$ CSAS25

UO2 108 W M 8.6

27GR̄OUPNDDF4 INFHOMMEDIUM

U-234 10.05 .7812 .5

U-235 10.0.00374561 END

U-238 1 0.0 3.218-5 END

H 10.0 .0575523 END

010.0 .03644737 END

CARBONSTEEL 21.0 END

AL $30.05 .54-4 \quad$ END

CA $30.05 .30-3 \quad$ END

C $30.06 .59-3 \quad$ END

H 30.0 .0133 END 


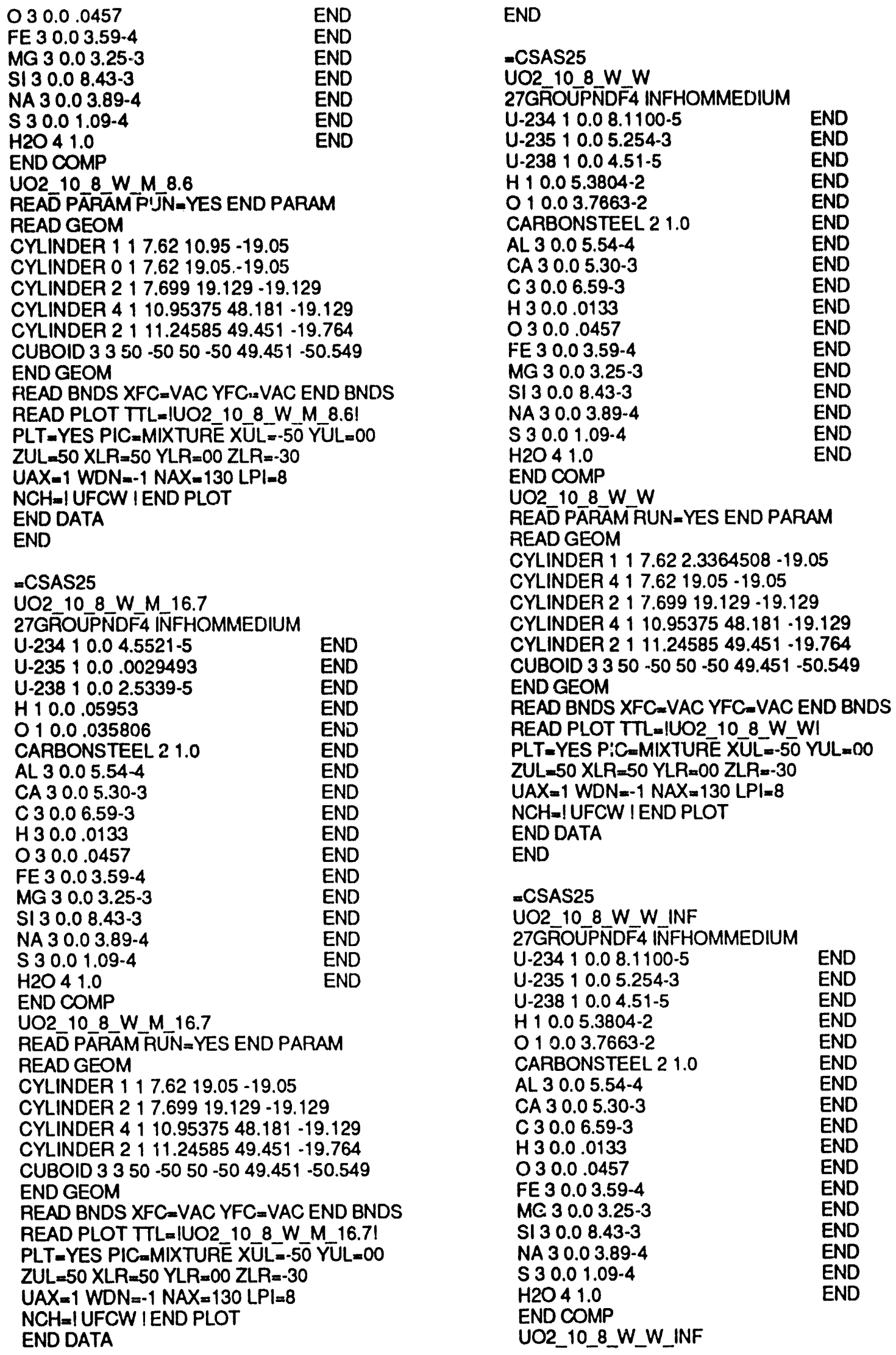


READ GEOM

UNIT 1

ZHEMICYL+X 117.622 .3364508 -19.05

ZHEMICYL $+X 417.622 P 19.05$

ZHEMICYL+X 217.699 2P 19.129

ZHEMICYL+X 4110.9537548 .181 -19.129

ZHEMICYL+X 2111.2458549 .451 -19.764

CUBOID $31105.5675030 .48-30.4849 .451$ $-35.004$

HOLE 2105.567500

HOLE 352.7837530 .480

HOLE $452.78375-30.480$

UNIT 2

ZHEMICYL-X 117.622 .3364508 -19.05

ZHEMICYL-X 417.62 2P19.05

ZHEMICYL-X $217.6992 P 19.129$

Z.HEMICYL-X 4110.9537548 .181 -19.129

ZHEMICYL-X $2111.2458549 .451-19.764$

UNIT 3

ZHEMICYL-Y 117.622 .3364508 -19.05

ZHEMICYL-Y 417.62 2P19.05

ZHEMICYL.Y 217.699 2P19.129

ZHEMICYL.Y $4110.9537548 .181-19.129$

ZHEMICYL-Y 2111.2458549 .451 -19.764

UNIT 4

ZHEMICYL+Y 117.622 .3364508 -19.05

ZHEMICYL+Y $417.622 P 19.05$

ZHEMICYL+Y $217.6992 P 19.129$

ZHEMICYL+Y $4110.9537548 .181-19.129$

ZHEMICYL+Y $2111.2458549 .451-19.764$

END GEOM

READ BNDS XFC $=S P E C$ YFC.SPEC END BNDS

READ PLOT TTL=IUO2 $108 \mathrm{~W} W$ INFI

PLT=YES PIC-MIXTURE XŪL $=0 \bar{Y} U \bar{L}=35$ ZUL $=0$

$X L R=110$ YLR $=-35$ ZLR $=0$

$U A X=1$ VDN $=-1 \quad N A X=130$ LPI $=8$

$N C H=\mid$ UF.W I END PLOT

END DATA

END

\section{-CSAS25}

UO2 10 FULL D D

27GR̄OUPNDFA INTHOMMEDIUM

U-234 $10.08 .1100-5$

U-235 $10.05 .254-3$

U.238 10.04 .51 .5

H $10.05 .3804-2$

O $10.03 .7663-2$

CARBONSTEEL 21.0

AL. $30.05 .54-4$

CA $30.05 .30-3$

C $30.06 .59-3$

H 30.0 .0133

030.0 .0457

FE $30.03 .59-4$

MG $30.03 .25-3$

Si $30.08 .43-3$

NA $30.03 .89-4$

S $30.01 .09-4$

$\mathrm{H} 2 \mathrm{O} 41.0$

END COMP

UO2 10 FULL D D

REA $\bar{D}$ PARAM $\bar{R} U \bar{N}=$ YES END PARAM

READ GEOM
CYLINDER 117.6219 .05 -19.05

CYLINDER 217.699 19.129 -19.129

CYLINDER $0110.9537548 .181-19.129$

CYLINDER $2111.2458549 .451-19.764$

CUBOID $3350 \cdot-5050 \cdot 50 \quad 49.451 \cdot 50.549$

END GEOM

READ BNDS XFC.VAC YFC.VAC END BNDS

READ PLOT TTL-IUO2 10 FULL. D DI

PLT=YES PIC=MIXTURE XÜL $=-50$ YÜL $=00$

$Z U L=50$ XLR $=50$ YLR $=00$ ZLR $=-30$

$U A X=1$ WDN $=-1 \quad N A X=130 \mathrm{LPI}=8$

$\mathrm{NCH}_{2}$ IUFCW IEND PLOT

END DATA

END

- CSAS25

UO2_10_FULL_D_D_INF

27GR̄OUPNDFA INFḦOMMEDIUM

U.234 $10.08 .1100-5$

U-235 $10.05 .254-3$

U.2381 0.04 .51 .5

H $10.05 .3804-2$

$010.03 .7663-2$

CARBONSTEEL 21.0

AL 30.05 .54 .4

CA $30.05 .30-3$

C 30.06 .59 .3

H 30.0 .0133

030.0 .0457

FE $30.03 .59-4$

MG $30.03 .25-3$

SI $30.08 .43-3$

NA 30.03 .89 .4

S $30.01 .09-4$

$\mathrm{H} 2 \mathrm{O} 41.0$

END COMP

UO2 10 FULL D D INF

READ GEOM

UNIT 1

ZHEMICYL+X $117.622 P 19.05$

ZHEMICYL+X 217.699 2P19.129

ZHEMICYL+X $0110.9537548 .181-19.129$

ZHEMICYL+X $2111.2458549 .451-19.764$

CUBOID $31105.5675030 .48 \cdot 30.4849 .451$

$-35.004$

HOLE 2105.567500

HOLE 352.7837530 .480

HOLE $452.78375-30.480$

UNIT 2

ZHEMICYL-X $117.622 P 19.05$

ZHEMICYL.X 217.699 2P19.129

ZHEMICYL-X $0110.9537548 .181-19.129$

ZHEMICYL-X $2111.2458549 .451-19.764$

UNIT 3

ZHEMICYL-Y 117.62 2P19.05

ZHEMICYL.Y $217.6992 P 19.129$

ZHEMICYL-Y 0110.9537548 .181 -19.129

ZHEMICYL-Y $2111.2458549 .451-19.764$

UNIT 4

ZHEMICYL+Y 117.62 2P19.05

ZHEMICYL+Y 217.699 2P19.129

ZHEMICYL+Y 0110.9537548 .181 -19.129

ZHEMICYL+Y $2111.2458549 .451-19.764$ END GEOM 
READ BNDS XFC=SPEC YFC SPEC END BNDS READ PLOT TTL=IUO2 10 FULL D D INFI PLT-YES PIC-MIXTURE XUL $=0$ YUL -35 ZUL $=0$ $X L R=110$ YLR $=35$ ZLR=0

$U A X=1$ VDN $=1$ NAX $=130$ LPI $=8$

$N C H=\mid$ UF.W I END PLOT

END DATA

END

\begin{tabular}{|c|c|}
\hline $\begin{array}{l}\text { CSAS25 } \\
\text { UO2 10 FULL_W D } \\
\text { 27GROUPNDF4 INFHOMMEDIUM } \\
\text { U-234 } 10.08 .1100-5 \\
\text { U-235 } 10.05 .254-3 \\
\text { U-238 } 10.04 .51-5 \\
\text { H } 10.05 .3804-2 \\
\text { O } 10.03 .7663-2 \\
\text { CARBONSTEEL } 21.0 \\
\text { AL } 30.05 .54-4 \\
\text { CA } 30.05 .30-3 \\
\text { C } 30.06 .59-3 \\
\text { H } 30.0 .0133 \\
\text { O } 30.0 .0457 \\
\text { FE } 30.03 .59-4 \\
\text { MG } 30.03 .25-3 \\
\text { SI } 30.08 .43-3 \\
\text { NA } 30.03 .89-4\end{array}$ & \\
\hline
\end{tabular}

\section{END COMP}

UO2 10 FULL W

READ PATAM $\bar{R} U \bar{N}=Y E S$ END PARAM

READ GEOM

CYLINDER $117.6219 .05-19.05$

CYLINDER $217.69919 .129-19.129$

CYLINDER $4110.9537548 .181-19.129$

CYLINDER 2111.2458549 .451 -19.764

CUBOID $3350-5050-50 \quad 49.451$ - 50.549

END GEOM

READ BNDS XFC=VAC YFC.VAC END BNDS

READ PLOT $T T L=1 U O 210$ FULL $W$ DI

PLT $=$ YES PIC $=M I X T U R \bar{E} X \bar{U} L=-5 \overline{0}$ YŨL $=00$

$Z U L=50$ XLR $=50$ YLR $=00$ ZLR $=-30$

$U A X=1$ WDN $=-1 \quad N A X=130$ LPI $=8$

$N C H=\mid$ UFCW $\mid$ END PLOT

END DATA

END

\section{-CSAS25}

UO2 15_5_D_D

27GR̄OUPN̄DFA INFHOMMEDIUM

U.234 1 0.0 5.99-5

U-235 10.0 .003878

U-238 10.03 .33 .5

H 10.0 .059566

010.0 .037725

CARBONSTEEL 21.0

AL $30.05 .54-4$

CA $30.05 .30-3$

C $30.06 .59-3$

H 30.0 .0133

O 30.0 .0457

FE $30.03 .59-4$

MG $30.03 .25-3$
SI $30.08 .43-3$

END

NA $30.03 .89-4$

END

S $30.01 .09-4$

END

$\mathrm{H} 2 \mathrm{O} 41.0$

END COMP

UO2 155 D D

READ PATAAM RUN= YES END PARAM

READ GEOM

CYLINDER 117.62 -9397338 - 19.05

CYLINDER $017.6219 .05-19.05$

CYLINDER $217.69919 .129-19.129$

CYLINDER $0110.9537548 .181-19.129$

CYLINDER $2111.2458549 .451-19.764$

CUBOID $3350-5050-50 \quad 49.451-50.549$

END GEOM

READ BNDS XFC=VAC YFC=VAC END BNDS

READ PLOT TTL $=1$ UO2 $155 \mathrm{D}$ DI

$P L T=Y E S$ PIC $=M I X T U R \bar{E}$ XÜL $=-\overline{50}$ YUL $=00$

$Z U L=50$ XLR $=50$ YLR $=00$ ZLR $=-30$

$U A X=1$ WDN $=-1 \quad N A X=130 \quad L P I=8$

$\mathrm{NCH}=|\mathrm{UFCW}| \mathrm{END}$ PLOT

END DATA

END

-CSAS25

UO2 155 D D INF

27GR̄OUPNDDF 4 INFHOMMEDIUM

U.234 $10.05 .99-5$

U-235 10.0 .003878

U.238 $10.03 .33-5$

H 10.0 .059566

010.0 .037725

CARBONSTEEL 21.0

AL $30.05 .54-4$

CA $30.05 .30-3$

C $30.06 .59-3$

H 30.0 .0133

030.0 .0457

FE $30.03 .59-4$

MG $30.03 .25-3$

SI $30.08 .43-3$

NA $30.03 .89-4$

S $30.01 .09-4$

END

$\mathrm{H} 2 \mathrm{O} 41.0$

END COMP

UO2 155 D D INF

READ GEOM

UNIT 1

ZHEMICYL+X $117.62-.9397338$-19.05

ZHEMICYL+X $017.622 P 19.05$

ZHEMICYL+X $217.6992 P 19.129$

ZHEMICYL+X $0110.9537548 .181-19.129$

ZHEMICYL+X $2111.2458549 .451-19.764$

CUBOID $31105.5675030 .48-30.4849 .451$

$-35.004$

HOLE 2105.567500

HOLE 352.7837530 .480

HOLE $452.78375-30.480$

UNIT 2

ZHEMICYL-X $117.62-9397338-19.05$

ZHEMICYL-X $017.622 P 19.05$

ZHEMICYL-X 217.699 2P19.129

ZHEMICYL-X 0110.9537548 .181 -19.129

ZHEMICYL-X 2111.2458549 .451 -19.764 
UNIT 3

ZHEMICYL-Y $117.62 \cdot .9397338-19.05$

ZHEMICYL-Y 017.62 2P19.05

ZHEMICYL-Y 217.699 2P19.129

ZHEMICYL-Y O $110.9537548 .181-19.129$

ZHEMICYL.Y $2111.2458549 .451-19.764$

UNIT 4

ZHEMICYL+Y 117.62 -.9397338 -19.05

ZHEMICYL+Y $017.622 P 19.05$

ZHEMICYL+Y $217.6992 P 19.129$

ZHEMICYL+Y $0110.9537548 .181 \cdot 19.129$

ZHEMICYL+Y $2111.2458549 .451-19.764$

\section{END GEOM}

READ BNDS XFC-SPEC YFC-SPEC END BNDS

READ PLOT TTL $=$ !UO2 15 5_D_D INFI

$P L T=Y E S P I C=M I X T U R E X \bar{U}=0 Y Y U L=35 Z U L=0$

$X L R=110 \quad Y L R=-35 \quad Z L R=0$

$U A X=1$ VDN $=-1 \quad N A X=130 \quad L P \mid=8$

$\mathrm{NCH}=\mid$ UF.W $\mid$ END PLOT

END DATA

END

\section{-CSAS25}

UO2 $155 \mathrm{~W} D$

27GR̄OUPNDF 4 INFHOMMEDIUM

U-234 $10.05 .99-5$

U-235 10.0 .003878

END

$\mathrm{U}-238$ 1 $0.03 .33-5$

END

H 10.0 .059566

010.0 .037725

CARBONSTEEL 21.0

AL 3 0.0 5.54-4

CA $30.05 .30-3$

C $30.06 .59-3$

H 30.0 .0133

O 30.0 .0457

FE $30.03 .59-4$

MG $30.03 .25-3$

SI $30.08 .43-3$

NA $30.03 .89-4$

S $30.01 .09-4$

$\mathrm{H} 2 \mathrm{O} 41.0$

END COMP

UO2 15_5 W_D

READ PARAM RUN=YES END PARAM

READ GEOM

CYLINDER $117.62-9397338-19.05$

CYLINDER $017.6219 .05-19.05$

CYLINDER $217.69919 .129-19.129$

CYLINDER $4110.9537548 .181-19.129$

CYLINDER $2111.2458549 .451-19.764$

CUBOID $3350 \quad-5050 \quad-5049.451 \quad-50.549$

END GEOM

READ BNDS XFC $=V A C$ YFC $=V A C$ END BNDS

READ PLOT TTLIUO2 $155 \mathrm{~W}$ DI

PLT $=Y E S$ PIC $=M I X T U R \overline{R E} X \bar{U}=-50$ YUL $=00$

$Z U L=50$ XLR $=50$ YLR $=00$ ZLR $=-30$

$U A X=1 W D N=-1 \quad N A X=130 L P \mid=8$

$\mathrm{NCH}=$ I UFCW IEND PLOT

END DATA

END

-CSAS25

UO2_15_5_W_D_H2O
27GROUPNDF4 INFHOMMEDIUM

U.234 $10.05 .99-5$

U.235 10.0 .003878

END

U-238 $10.03 .33-5$

H 10.0 .059566

010.0 .037725

CARBONSTEEL 21.0

AL. $30.05 .54-4$

CA $30.05 .30-3$

C $30.06 .59-3$

H 30.0 .0133

O 30.0 .0457

FE $30.03 .59-4$

MG $30.03 .25-3$

SI $30.08 .43-3$

NA $30.03 .89-4$

S $30.01 .09-4$

END

END

END

END

END

END

END

END

END

END

END

END

END

END

$\mathrm{H} 2 \mathrm{O} 41.0$

END COMP

UO2 155 W D H2O

REA $\bar{D}$ PARAM $\bar{R} \bar{N}=$ =VES END PARAM

READ GEOM

CYLINDER $117.62-.9397338$-19.05

CYLINDER $017.6219 .05-19.05$

CYLINDER $217.69919 .129-19.129$

CUBOID $4328-2828-2839-39$

END GEOM

READ BNDS XFC $=V A C$ YFC $=$ VAC END BNDS

READ PLOT $T T L=! U O 2$ 15_5_W_D_H2O!

PLT $=Y E S$ PIC $=M I X T U R E$ XU $=-5 \overline{0}$ YUL $=00$

$Z U L=50 X L R=50$ YLR $=00$ ZLR $=-30$

$U A X=1$ WDN $=-1$ NAX $=130 \mathrm{LPI}=8$

NCH=I UFCW IEND PLOT

END DATA

END

-CSAS25

UO2_15_5_W_D_INF

27GR̄OUPÑDF4 INFHOMMEDIUM

U-234 $10.05 .99-5$

$U-23510.0 .003878$

U-238 $10.03 .33-5$

H 10.0 .059566

O 10.0 .037725

CARBONSTEEL 21.0

AL 30.0 5.54-4

CA $3005.30-3$

C $30.06 .59-3$

H 30.0 .0133

O 30.0 .0457

FE $30.03 .59-4$

MG $30.03 .25-3$

SI $30.08 .43-3$

NA $30.03 .89-4$

S $30.01 .09-4$

$\mathrm{H} 2 \mathrm{O} 41.0$

END COMP

UO2 15_5 W_D_INF

READ GEOM

UNIT 1

ZHEMICYL $+X 117.62-.9397338-19.05$

ZHEMICYL+X $017.622 P 19.05$

ZHEMICYL+X $217.6992 P 19.129$

ZHEMICYL+X $4110.9537548 .181-19.129$ 
ZHEMICYL+X $2111.2458549 .451-19.764$ CUBOID 31105.5675030 .48 -30.48 49.451 $-35.004$

HOLE 2105.567500

HOLE 352.7837530 .480

HOLE $452.78375-30.480$

UNIT 2

ZHEMICYL-X $117.62-.9397338-19.05$

ZHEMICYL-X $017.622 \mathrm{P} 19.05$

ZHEMICYL-X 217.699 2P 19.129

ZHEMICYL-X $4110.9537548 .181-19.129$

ZHEMICYL-X $2111.2458549 .451-19.764$

UNIT 3

ZHEMICYL-Y 117.62 -.9397338 -19.05

Z.HEMICYL-Y 017.62 2P19.05

ZHEMICYL-Y 217.699 2P19.129

ZHEMICYL-Y $4110.9537548 .181-19.129$

ZHEMICYL-Y $2111.2458549 .451-19.764$

UNIT 4

ZHEMICYL+Y $117.62-.9397338-19.05$

ZHEMICYL+Y $017.622 P 19.05$

ZHEMICYL+Y $217.6992 P 19.129$

ZHEMICYL+Y $4110.9537548 .181-19.129$

ZHEMICYL+Y $2111.2458549 .451-19.764$

END GEOM

READ BNDS XFC=SPEC YFC=SPEC END BNDS

READ PLOT TTL=!UO2 $155 \mathrm{~W} D$ INF!

$P L T=Y E S$ PIC $=M I X T U R \bar{E} \times \bar{U} L=0 \quad \bar{Y} \bar{L}=35 Z U L=0$

$X L R=110 Y L R=-35 \quad Z L R=0$

$U A X=1$ VDN $=-1 \quad N A X=130 \quad \mathrm{LPI}=8$

$N C H=$ ! UF.W ! END PLOT

END DATA

END

\section{-CSAS25}

UO2 15 SW M 5

27GR̄OUPNDF4 INFHOMMEDIUM

U-234 $10.04 .6906-5$

U-235 10.0 .00303892

U-238 $10.02 .6109-5$

H 10.0 .061144

010.0 .0367959

CARBONSTEEL 21.0

AL $30.05 .54-4$

CA $30.05 .30-3$

C $30.06 .59-3$

H 30.0 .0133

030.0 .0457

FE $30.03 .59-4$

MG $30.03 .25-3$

SI $30.08 .43-3$

NA $30.03 .89-4$

S $30.01 .09-4$

$\mathrm{H} 2 \mathrm{O} 41.0$

END COMP

UO2 15_5_W_M 5

READ PARAMM $\bar{R} \bar{N}=$ YES END PARAM

READ GEOM

CYLINDER $117.624 .060266-19.05$

CYLINDER $017.6219 .05-19.05$

CYLINDER $217.69919 .129-19.129$

CYLINDER $4110.9537548 .181-19.129$

CYLINDER $2111.2458549 .451-19.764$

CUBOID $3350-5050-5049.451-50.549$

\section{END GEOM}

READ BNDS $X F C=V A C$ YFC $=V A C$ END BNDS

READ PLOT TIL $=1 \mathrm{UO2} 155 \mathrm{~W} \mathrm{M} 5$ !

PLT $=Y E S$ PIC $=M I X T U R E$ XUL $=-50$ YUL $=00$

$Z U L=50$ XLR $=50$ YLR $=00$ ZLR $=-30$

$U A X=1$ WDN=-1 NAX=130 LPI $=8$

$\mathrm{NCH}=$ ! UFCW ! END PLOT

END DATA

END

$=$ CSAS25

UO2 $155 W-M-15$

27GR̄OUPNDFA INFHOMMEDIUM

U-234 $10.03 .2739-5$

U-235 10.0 .0021211

U-238 $10.01 .8224-5$

H 10.0 .06287

010.0 .0357791

CARBONSTEEL 21.0

AL $30.05 .54-4$

CA $30.05 .30-3$

C $30.06 .59-3$

H 30.0 .0133

O 30.0 .0457

FE $30.03 .59-4$

MG $30.03 .25-3$

SI $30.08 .43-3$

NA $30.03 .89-4$

S $30.01 .09-4$

$\mathrm{H} 2 \mathrm{O} 41.0$

END COMP

UO2 15 5 W M 15

READ PATRAM $\bar{R} \overline{U N}=$ YES END PARAM

READ GEOM

CYLINDER 117.6214 .020928 -19.05

CYLINDER $017.6219 .05-19.05$

CYLINDER 217.699 19.129-19.129

CYLINDER $4110.9537548 .181-19.129$

CYLINDER $2111.2458549 .451-19.764$

CUBOID $3350 \quad-5050-50 \quad 49.451 \quad-50.549$

END GEOM

READ BNDS XFC $=V A C$ YFC $=V A C$ END BNDS

READ PLOT TTL=!UO2 $155 \mathrm{~W} M$ 15!

$P L T=Y E S$ PIC $=M I X T U R \bar{E} \times \overline{U L}=-5 \bar{Y} \overline{Y U L}=00$

$Z U L=50 X L R=50$ YLR $=00 Z L R=-30$

$U A X=1$ WDN $=-1 \quad N A X=130 L P I=8$

$\mathrm{NCH}=$ ! UFCW ! END PLOT

END DÁTA

END

$=$ CSAS25

UO2 155 W M 20

27GR̄OUPN̄DF4 INFHOMMEDIUM

U-238 $10.01 .5837-5$ END

H 10.0 .06339238 END

$010.0 .03547136 \quad$ END

CARBONSTEEL 21.0 END

AL $30.05 .54-4$ END

CA $30.05 .30-3 \quad$ END

C $30.06 .59-3$ END

H3 0.0 .0133 END

030.0 .0457 END 


\begin{tabular}{|c|c|}
\hline 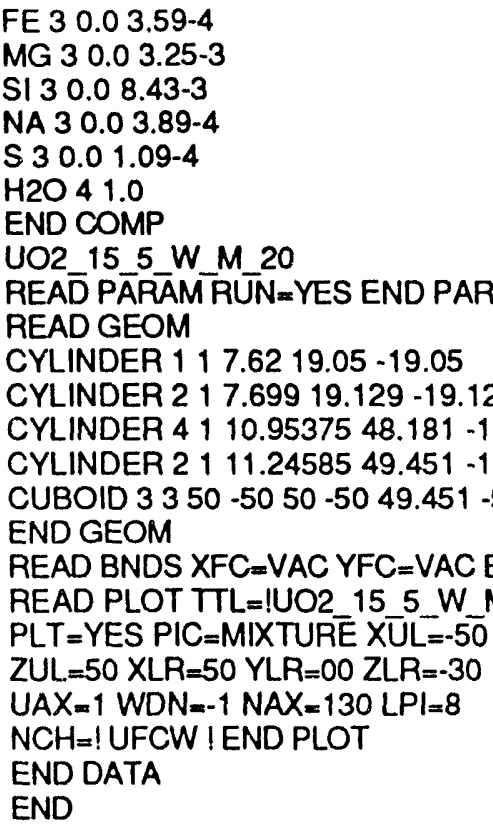 & $\begin{array}{l}\text { END } \\
\text { END } \\
\text { END } \\
\text { END } \\
\text { END } \\
\text { END } \\
\text { AM } \\
\\
9.129 \\
9.764 \\
50.549 \\
\text { END BNDS } \\
\text { Y_20! } \\
\text { YUL }=00\end{array}$ \\
\hline
\end{tabular}

\section{$=$ CSAS25}

UO2_15_5 W W INF

27GR̄OUPND $\overline{4} 4$ INFHOMMEDIUM

U-234 $10.05 .99-5$

U-235 10.0 .003878

U-238 $10.03 .33-5$

H 10.0 .059566

010.0 .037725

CARBONSTEEL 21.0

$A L 30.05 .54-4$

CA $30.05 .30-3$

C $30.06 .59-3$

H 30.0 .0133

030.0 .0457

FE $30.03 .59-4$

MG $30.03 .25-3$

SI $30.08 .43-3$

NA $30.03 .89-4$

S $30.01 .09-4$

$\mathrm{H} 2 \mathrm{O} 41.0$

END COMP

UO2 $155 \mathrm{~W} W$ INF

READ GE $\overline{G M}$

UNIT 1

ZHEMICYL+X $117.62-9397338-19.05$

ZHEMICYL+X $417.622 P 19.05$

ZHEMICYL+X 217.699 2P19.129

ZHEMICYL+X $4110.9537548 .181-19.129$

ZHEMICYL+X $2111.2458549 .451-19.764$ CUBOID $31105.5675030 .48-30.4849 .451$ $-35.004$

HOLE 2105.567500

HOLE 352.7837530 .480

HOLE $452.78375-30.480$

UNIT 2

ZHEMICYL-X $117.62-9397338$-19.05

ZHEMICYL-X 417.62 2P19.05

ZHEMICYL-X $217.6992 P 19.129$

ZHEMICYL-X 411 10.35375 48.181 -19.129
ZHEMICYL-X $2111.2458549 .451-19.764$

UNTT 3

ZHEMICYL-Y 117.62 -.9397338 -19.05

ZHEMICYL-Y 417.62 2P19.05

ZHEMICYL-Y 217.699 2P19.129

ZHEMICYL-Y $4110.9537548 .181-19.129$

ZHEMICYL-Y 2111.2458549 .451 -19.764

UNIT 4

ZHEMICYL+Y 117.62 -.9397338 -19.05

ZHEMICYL+Y $417.622 P 19.05$

ZHEMICYL+Y 217.699 2P19.129

ZHEMICYL+Y $4110.9537548 .181-19.129$

ZHEMICYL+Y $2111.2458549 .451-19.764$

END GEOM

READ BNDS XFC=SPEC YFC=SPEC END BNDS

READ PLOT TTL=!UO2 $155 \mathrm{~W} W$ INF!

$P L T=Y E S P I C=M I X T U R E X \bar{U} L=1 \overline{10}$ YUL $=-35$

$Z U L=0 X L R=0 Y L R=35$ ZLR $=0$

$V A X=1$ UDN $=-1 \quad \mathrm{NAX}=130 \mathrm{LPI}=8$

$\mathrm{NCH}=! U F . W \mid E N D$ PLOT

END DATA

END

\section{=CSAS25}

UO2 15\&W D

27GR̄OUPND $\overline{\text { F } 4}$ INFHOMMEDIUM

U-234 $10.05 .99-5$

U-235 10.0 .003878

END

END

U-238 1 0.0 3.33-5 END

H 10.0 .059566 END

$010.0 .037725 \quad$ END

CARBONSTEEL 21.0 END

AL $30.05 .54-4$ END

CA $30.05 .30-3 \quad$ END

C $30.06 .59-3 \quad$ END

H3 0.0 .0133 END

030.0 .0457 END

FE $30.03 .59-4 \quad$ END

MG $30.03 .25-3$ END

SI $30.08 .43-3 \quad$ END

NA $30.03 .89-4$ END

$S 30.01 .09-4 \quad$ END

H2O 41.0 END

END COMP

UO2 158 W D

READ PA $\bar{A} \overline{R A M} \bar{R} U N=Y E S$ END PARAM

READ GEOM

CYLINDER $117.629 .926426-19.05$

CYLINDER $017.6219 .05-19.05$

CYLINDER 2'1 7.699 19.129-19.129

CYLINDER $4110.9537548 .181-19.129$

CYLINDER $2111.2458549 .451-19.764$

CUBOID $3350-5050-5049.451-50.549$

END GEOM

READ BNDS XFC $=V A C$ YFC $=V A C$ END BNDS

READ PLOT TTL=!UO2_15_8_W_D!

$P L T=Y E S$ PIC $=M I X T U R \bar{E} \times \bar{U}=-50$ YUL $=00$

$Z U L=50 \times L R=50$ YLR $=00 \quad Z L R=-30$

$U A X=1$ WDN $=-1 \quad N A X=130 \quad L P I=8$

$\mathrm{NCH}=$ ! UFCW ! END PLOT

END DATA

END

-CSAS25 
UO2 15 FULL D D

27GR̄OUPNDF" INFHOMMEDIUM

U.234 $10.05 .99-5$

U.235 10.0 .003878

END

U-238 $10.03 .33-5$

END

H 10.0 .059566

010.0 .037725

CARBONSTEEL 21.0

END

END

END

END

AL 30.05 .54 .4

CA $30.05 .30-3$

END

C $30.06 .59-3$

END

H 30.0 .0133

END

END

FE $30.03 .59-4$

END

MG $30.03 .25-3$

END

SI $30.08 .43-3$

END

END

S $30.01 .09-4$ END

$\mathrm{H} 2 \mathrm{O} 41.0$

END COMP

UO2 15 FULL D D

REA $\bar{D}$ PA $\bar{A} R A M \bar{R} \bar{U} \bar{N}=Y E S$ END PARAM READ GEOM

CYLINDER $117.6219 .05 \cdot 19.05$

CYLINDER $217.69919 .129 \cdot 19.129$

CYLINDER $0110.9537548 .181-19.129$

CYLINDER $21\lceil 1.2458549 .451-19.764$

CUBOID $3350-5050-5049.451-50.549$

END GEOM

READ BNDS XFC $=V A C$ YFC $=V A C$ END BNDS

READ PLOT TTL $=$ !UO2 15 FULL D D!

PLT $=$ YES PIC $=$ MIXTURE XULL $=-5 \overline{0}$ YULL $=00$

$Z U L=50$ XLR $=50$ YLR $=00$ ZLR $=-30$

$U A X=1$ WDN $=-1 \quad N A X=130 \quad L P I=8$

$\mathrm{NCH}=$ ! UFCW IEND PLOT

END DATA

END

=CSAS25

UO2 15 FULL_D_D INF 27GR̄OUPNDFA INFHOMMEDIUM

U-234 $10.05 .99-5$

U-235 10.0 .003878

END

U-238 $10.03 .33-5$

H 10.0 .059566

O 10.0 .037725

CARBONSTEEL 21.0

AL $30.05 .54-4$

CA $30.05 .30-3$

C $30.06 .59-3$

H3 0.0 .0133

030.0 .0457

FE $30.03 .59-4$

MG $30.03 .25-3$

SI $30.08 .43-3$

NA 30.03 .89 .4

S $30.01 .09-4$

$\mathrm{H} 2 \mathrm{O} 41.0$

END COMP

UO2 15 FULL D D INF

READ GEOM

UNIT 1

ZHEMICYL+X $117.622 \mathrm{P} 19.05$

ZHEMICYL+X $217.6992 P 19.129$
ZHEMICYL+X $0110.9537548 .181-19.129$

ZHEMICYL+X $2111.2458549 .451-19.764$ CUBOID $31105.5675030 .48-30.4849 .451$

$-35.004$

HOLE 2105.567500

HOLE 352.7837530 .480

HOLE $452.78375-30.480$

UNIT 2

ZHEMICYL-X 117.62 2P19.05

ZHEMICYL-X $217.6992 P 19.129$

ZHEMICYL-X $0110.9537548 .181-19.129$

ZHEMICYL-X $2111.2458549 .451-19.764$

UNIT 3

ZHEMICYL-Y 117.62 2P19.05

ZHEMICYL-Y 217.699 2P19.129

ZHEMICYL-Y $0110.9537548 .181-19.129$

ZHEMICYL-Y 2111.2458549 .451 -19.76.4

UNIT 4

ZHEMICYL+Y $117.622 \mathrm{P} 19.05$

ZHEMICYL+Y 217.699 2P19.129

ZHEMICYL+Y 0110.9537548 .181 -19.129

ZHEMICYL+Y $2111.2458549 .451-19.764$

END GEOM

READ BNDS XFC $=S P E C$ YFC $=S P E C$ END BNDS

READ PLOT TTLE!UO2 15 FULL D D INFI

PLT $=Y E S$ PIC $=$ MIXTURE $X \bar{U} L=0$ YUL $=3 \overline{5} Z U L=0$

$X L R=110 \quad Y L R=-35 Z L R=0$

$U A X=1$ VDN $=-1 \quad$ NAX $=130 \quad L P I=8$

$\mathrm{NCH}=$ ! UF.W ! END PLOT

END DATA

END

-CSAS25

UO2 15 FULL W D

27GR̄OUPNDFA INFHOMMEDIUM

U-234 $10.05 .99-5$

U-235 10.0 .003878

$\mathrm{U}-23810.03 .33-5$

H 10.0 .059566

010.0 .037725

CARBONSTEEL 21.0

AL $30.05 .54-4$

CA $30.05 .30-3$

C $30.06 .59-3$

H 30.0 .0133

030.0 .0457

FE $30.03 .59-4$

MG $30.03 .25-3$

SI $30.08 .43-3$

NA $30.03 .89-4$

S $30.01 .09-4$

$\mathrm{H} 2 \mathrm{O} 41.0$

END COMP

UO2 15 FULL W D

REA $\bar{D}$ PA $\bar{A} A M \bar{R} U \bar{N}=Y E S$ END PARAM

READ GEOM

CYLINDER $117.6219 .05-19.05$

CYLINDER $217.69919 .129-19.129$

CYLINDER $4110.9537548 .181-19.129$

CYLINDER $2111.2458549 .451-19.764$

CUBOID $3350-5050-5049.451-50.549$

END GEOM

READ BNDS XFC $=V A C$ YFC $=$ VAC END BNDS

READ PLOT TTL=!UO2_15_FULL_W_D! 
$P L T=Y E S P I C=M I X T U R E X U L=-50$ YUL $=00$

$Z U L=50 X L R=50$ YLR $=00 \quad Z L R=-30$

$U A X=1 W D N=-1 \quad N A X=130 \quad L P I=8$

$\mathrm{NCH}=$ ! UFCW ! END PLOT

END DATA

END

-CSAS25

UO2 $651.2 \mathrm{~W} \mathrm{D}$

27GR'OUPNDF 4 INFHOMMEDIUM

U-234 $10.01 .540-5$

U-235 10.0 .001001

END

U-238 $10.08 .56-6$

END

H 10.0 .06661

010.0 .035354

CARBONSTEEL 21.0

AL. $30.05 .54-4$

CA $30.05 .30-3$

C $30.06 .59-3$

H 30.0 .0133

030.0 .0457

FE $30.03 .59-4$

MG $30.03 .25-3$

SI $30.08 .43-3$

NA $30.03 .89-4$

S $30.01 .09-4$

$\mathrm{H} 2 \mathrm{O} 41.0$

END COMP

UO2 $651.2 W$ D

READ PARAM RUN $=Y E S$ END PARAM

READ GEOM

CYLINDER 117.62 -2.207443 -19.05

CYLINDER $017.6219 .05-19.05$

CYLINDER $217.69919 .129-19.129$

CYLINDER $4110.9537548 .181-19.129$

CYLINDER $2111.2458549 .451-19.764$

CUBOID $3350-50 \quad 50-5049.451-50.549$

END GEOM

READ BNDS XFC $=V A C$ YFC $=V A C$ END BNDS

READ PLOT TTL $=1 U O 2651.2 \mathrm{~W}$ D!

$P L T=Y E S$ PIC $=M I X T U R \bar{E} X \bar{U} L=-50 \overline{Y U L}=00$

$Z U L=50$ XLR $=50$ YLR $=00$ ZLR $=-30$

$U A X=1 W D N=-1 \quad N A X=130 \quad L P \mid=8$

$\mathrm{NCH}=$ ! UFCW $\mid$ END PLOT

END DATA

END

\section{$=$ CSAS25}

UO2 65 2 D D

27GROUPNDDF4 INFHOMMEDIUM

U.234 $10.01 .540-5$

U-235 10.0 .001001

U-238 $10.08 .56-6$

H 10.0 .06661

010.0 .035354

CARBONSTEEL 21.0

AL $30.05 .54-4$

CA $30.05 .30-3$

C $30.06 .59-3$

H 30.0 .0133

030.0 .0457

FE $30.03 .59-4$

MG $30.03 .25-3$

SI $30.08 .43-3$
NA $30.03 .89-4$

END

S $30.01 .09-4$

$\mathrm{H} 2 \mathrm{O} 41.0$

END COMP

UO2 $652 \mathrm{D} D$

READ PARAM RUN=YES END PARAM

READ GEOM

CYLINDER 117.629 .020928 - 19.05

CYLINDER $017.6219 .05-19.05$

CYLINDER $217.69919 .129-19.129$

CYLINDER $0110.9537548 .181-19.129$

CYLINDER $2111.2458549 .451-19.764$

CUBOID $3350-5050-5049.451-50.549$

END GEOM

READ BNDS XFC=VAC YFC $=V A C$ END BNDS

READ PLOT TTL $=! U O 2652 \mathrm{D}$ D!

PLT $=$ YES PIC $=M I X T U R E$ XUL $=-50$ YUL $=00$

$Z U L=50 X L R=50$ YLR $=00 Z L R=-30$

$U A X=1$ WDN $=-1 \quad \mathrm{NAX}=130 \mathrm{LPI}=8$

$\mathrm{NCH}=$ ! UFCW ! END PLOT

END DATA

END

$=$ CSAS25

UO2_65_2_D_D H2O

27GROUP $\bar{N} \bar{F} 4$ INFHOMMEDIUM

U-234 $10.01 .540-5$

U-235 10.0 .001001

U-238 $10.08 .56-6$

H 10.0 .06661

010.0 .035354

CARBONSTEEL 21.0

AL $30.05 .54-4$

CA $30.05 .30-3$

C $30.06 .59-3$

H 30.0 .0133

030.0 .0457

FE $30.03 .59-4$

MG $30.03 .25-3$

SI $30.08 .43-3$

NA $30.03 .89-4$

S $30.01 .09-4$

$\mathrm{H} 2 \mathrm{O} 41.0$

END COMP

UO2 652 D D H2O

READ PA $\bar{A} \bar{A} \bar{M}$ RU $N=$ YES END PARAM

READ GEOM

CYLINDER $117.629 .020928-19.05$

CYLINDER $017.6219 .05-19.05$

CYLINDER $217.69919 .129-19.129$

CUBOID $4228-2828-2839-39$

END GEOM

READ BNDS XFC $=V A C$ YFC $=V A C$ END BNDS

READ PLOT TTL=!UO2 652 D D H2O!

$P L T=Y E S$ PIC $=$ MIXTURE XUL $=-50 \overline{Y U L}=00$

$Z U L=50 X L R=50 \quad Y L R=00 Z L R=-30$

$U A X=1$ WDN $=-1 \quad N A X=130 \quad L P I=8$

$\mathrm{NCH}=$ ! UFCW ! END PLOT

END DATA

END

$=$ CSAS25

UO2_65_2 D_D INF

27GR̄OUPN̄DF4 TNFHOMMEDIUM 


\begin{tabular}{|c|c|c|c|}
\hline 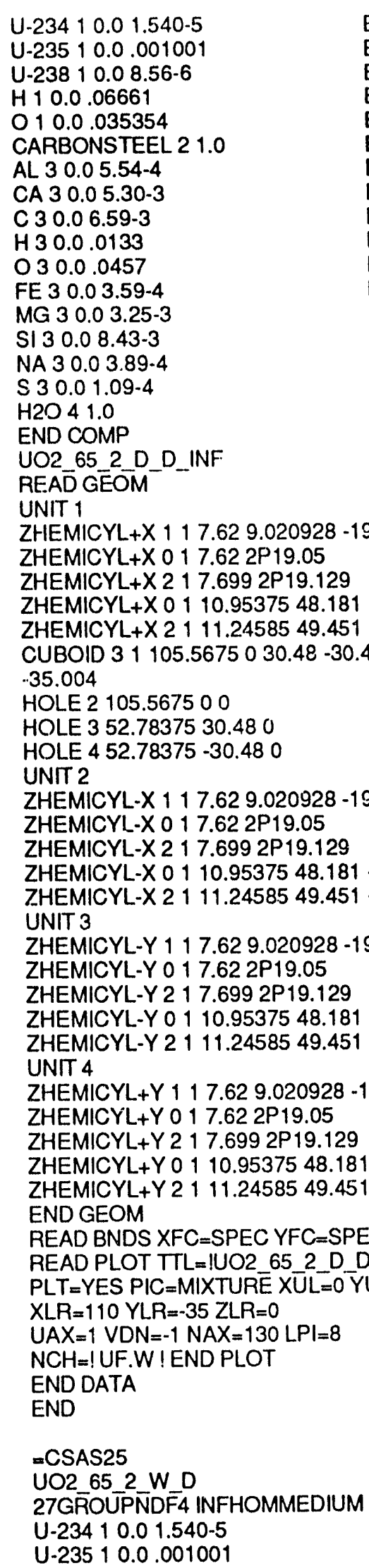 & 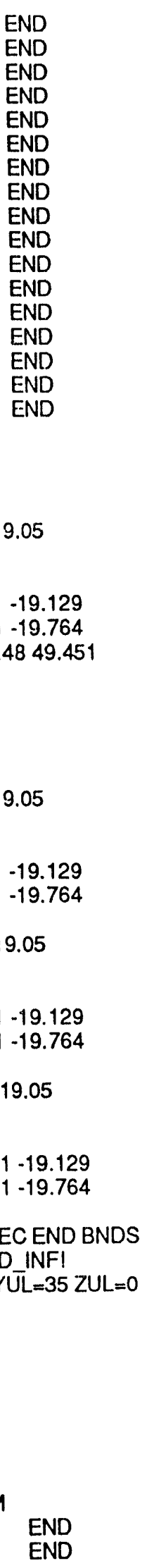 & 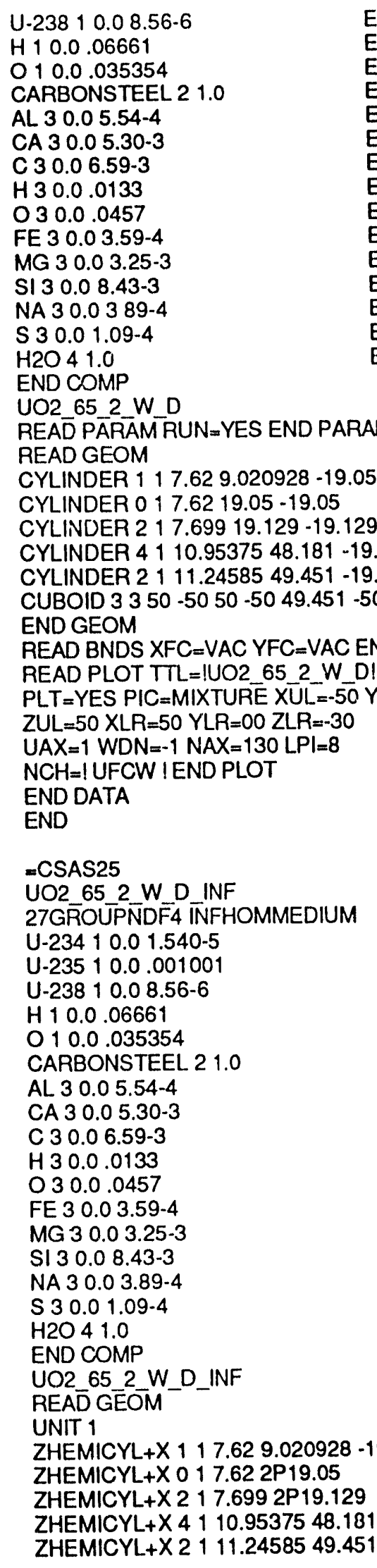 & $\begin{array}{l}\text { END } \\
\text { END } \\
\text { END } \\
\text { END } \\
\text { END } \\
\text { END } \\
\text { END } \\
\text { END } \\
\text { END } \\
\text { END } \\
\text { END } \\
\text { END } \\
\text { END } \\
\text { END } \\
\text { END } \\
\text { END } \\
\text { END }\end{array}$ \\
\hline
\end{tabular}


CUBOID $31105.5675030 .48-30.4849 .451$ $-35.004$

HOLE 2105.567500

HOLE 352.7837530 .480

HOLE $452.78375-30.480$

UNIT 2

ZHEMICYL-X 117.629 .020928 -19.05

ZHEMICYL-X $017.622 \mathrm{P} 19.05$

ZHEMICYL-X 217.699 2P19.129

ZHEMICYL-X 4110.9537548 .181 -19.129

ZHEMICYL-X $2111.2458549 .451-19.764$ UNIT 3

ZHEMICYL-Y 117.629 .020928 -19.05

ZHEMICYL-Y 017.62 2P19.05

ZHEMICYL-Y 217.699 2P19.129

ZHEMICYL-Y $4110.9537548 .181-19.129$

ZHEMICYL-Y $2111.2458549 .451-19.764$

UNIT 4

ZHEMICYL+Y 117.629 .020928 -19.05

$Z H E M I C Y L+Y 017.622 P 19.05$

ZHEMICYL+Y $217.6992 P 19.129$

ZHEMICYL+Y $4110.9537548 .181-19.129$

ZHEMICYL+Y $2111.2458549 .451-19.764$

END GEOM

READ BNDS XFC=SPEC YFC $=S P E C$ END BNDS

READ PLOT TTL=!UO2 $652 \mathrm{~W} D$ INF!

$P L T=Y E S P I C=M I X T U R E X \bar{U} L=0 \bar{Y} U \bar{L}=35 Z U L=0$

$X L R=110$ YLR $=-35 Z L R=0$

$U A X=1$ VDN $=-1 \quad N A X=130 \quad L P I=8$

$\mathrm{NCH}=\mid$ UF.W $\mid$ END PLOT

END DATA

END

\section{=CSAS25}

UO2 652 W M 7

27GR̄OUPN̄DFA INFHOMMEDIUM

U.234 $10.01 .2305 \mathrm{E}-05$

U-235 $10.08 .0101 E-04$

U-238 $10.06 .8496 \mathrm{E}-06$

H 10.00 .06665521

010.00 .03496793

CARBONSTEEL 21.0

AL $30.05 .54-4$

CA $30.05 .30-3$

C $30.06 .59-3$

H 30.0 .0133

030.0 .0457

FE $30.03 .59-4$

MG $30.03 .25-3$

SI $30.08 .43-3$

NA 30.03 .89 .4

S $30.01 .09-4$

$\mathrm{H} 2 \mathrm{O} 41.0$

END COMP

UO2 $652 \mathrm{~W} \cdot \mathrm{M} 7$

REA $\bar{D}$ PARAM $\bar{R} \bar{U}=$ Y $=$ ES END PARAM

READ GEOM

CYLINDER $117.6216 .02092807-19.05$

CYLINDER $017.6219 .05-19.05$

CYLINDER $217.69919 .129-19.129$

CYLINDER $4110.9537548 .181-19.129$

CYLINDER $2111.2458549 .451-19.764$

CUBOID $3350-5050-5049.451-50.549$ END GEOM
READ BNDS XFC $=V A C$ YFC $=V A C$ END BNDS

READ PLOT TTL=IUO2 $652 \mathrm{~W} M 71$

PLT $=$ YES PIC $=M I X T U R \bar{E}$ XÜL $=-5 \overline{0}$ YUL $=00$

$Z U L=50$ XLR $=50$ YLR $=00 \quad Z L R=-30$

$U A X=1$ WDN $=-1 \quad N A X=130 \quad L P I=8$

$\mathrm{NCH}=\mid$ UFCW IEND PLOT

END DATA

END

$=$ CSAS25

UO2 652 W M 8

27GROUPNDF4 INFHOMMEDIUM

U-234 10.01 .1964 E-05

U-235 $10.07 .7880 \mathrm{E}-04$

U-238 $10.06 .6597 E-06$

H 10.00 .06666085

010.00 .034925271

CARBONSTEEL 21.0

AL $30.05 .54-4$

CA $30.05 .30-3$

C $30.06 .59-3$

H3 0.0 .0133

030.0 .0457

FE $30.03 .59-4$

MG $30.03 .25-3$

SI $30.08 .43-3$

NA 30.03 .89 .4

S $30.01 .09-4$

END

END

END

END

END

END

END

END

END

END

END

END

END

END

END

END

$\mathrm{H} 2 \mathrm{O} 41.0$

END COMP

UO2 $652 W M 8$

READ PARAM RUUN=YES END PARAM

READ GEOM

CYLINDER 117.6217 .02092807 -19.05

CYLINDER $017.6219 .05-19.05$

CYLINDER 217.699 19.129-19.129

CYLINDER $4110.9537548 .181-19.129$

CYLINDER $2111.2458549 .451-19.764$

CUBOID $3350-5050-5049.451-50.549$

END GEOM

READ BNDS XFC=VAC YFC $=V A C$ END BNDS

READ PLOT TTL $=$ IUO2 $652 \mathrm{~W} M 81$

$P L T=Y E S$ PIC $=$ MIXTURE XÜL $=-50$ YUL $=00$

$Z U L=50 \quad X L R=50$ YLR $=00 \quad Z L R=-30$

$U A X=1$ WDN $=-1 \quad N A X=130 \quad \mathrm{LPI}=8$

$\mathrm{NCH}=$ ! UFCW IEND PLOT

END DATA

END

$=$ CSAS 25

UO2 $652 \mathrm{~W} M 10$

27GR̄OUPN̄DF4 INFHOMMEDIUM

U-234 $10.01 .1330 E-05$

U-235 $10.07 .3750 E-04$

U-238 $10.06 .3065 E-06$

H 10.00 .06667133

010.00 .03484594

CARBONSTEEL 21.0

AL $30.05 .54-4$

CA $30.05 .30-3$

C $30.06 .59-3$

H 30.0 .0133

030.0 .0457

FE $30.03 .59-4$

END

END

END

END

END

END

END

END

END

END

END

END 


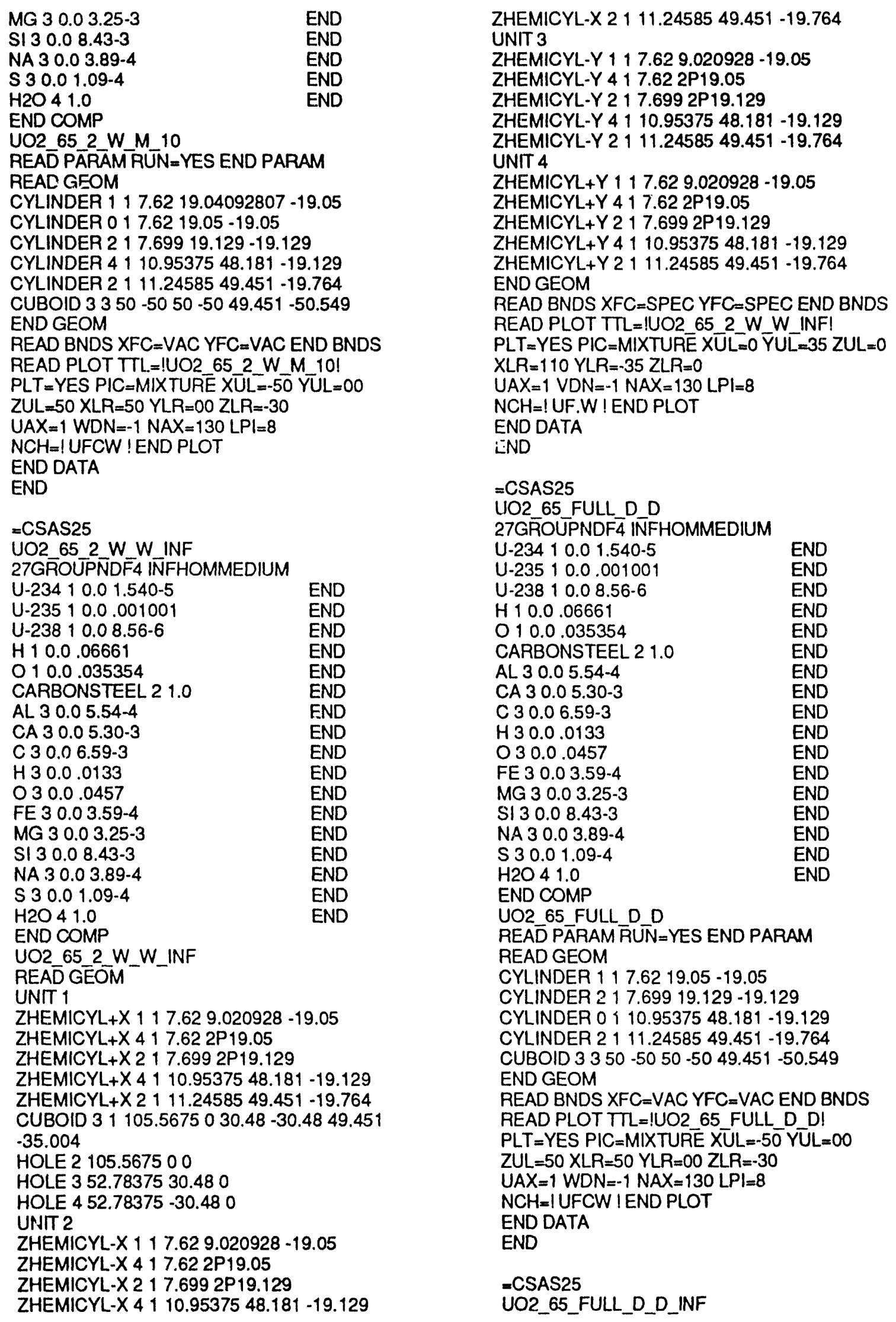


27GROUPNDF4 INFHOMMEDIUM

U-234 $10.01 .540-5$

U-235 10.0 .001001

U-238 $10.08 .56-6$

H 10.0 .06661

O 10.0 .035354

CARBONSTEEL 21.0

AL 3 0.0 5.54-4

CA $30.05 .30-3$

C $30.06 .59-3$

H 30.0 .0133

O 30.0 .0457

FE $30.03 .59-4$

MG $30.03 .25-3$

SI $30.08 .43-3$

NA $30.03 .89-4$

S $30.01 .09-4$

$\mathrm{H} 2 \mathrm{O} 41.0$

END COMP

UO2_65_FULL_D_D_INF

READ GEOM

UNIT 1

ZHEMICYL+X $117.622 P 19.05$

ZHEMICYL+X 217.699 2P19.129

ZHEMICYL+X $0110.9537548 .181-19.129$

ZHEMICYL+X $2111.2458549 .451-19.764$

CUBOID $31105.5675030 .48-30: 4849.451$

$-35.004$

HOLE 2105.567500

HOLE 352.7837530 .480

HOLE $452.78375-30.480$

UNIT 2

ZHEMICYL-X 117.62 2P19.05

ZHEMICYL-X 217.699 2P19.129

ZHEMICYL-X $0110.9537548 .181-19.129$

ZHEMICYL-X $2111.2458549 .451-19.764$

UNIT 3

ZHEMICYL-Y 117.62 2P19.05

ZHEMICYL-Y $217.6992 P 19.129$

ZHEMICYL-Y $0110.9537548 .181-19.129$

ZHEMICYL-Y $2111.2458549 .451-19.764$

UNIT 4

ZHEMICYL+Y $117.622 P 19.05$

ZHEMICYL+Y $217.6992 P 19.129$

ZHEMICYL+Y $0110.9537548 .181-19.129$

ZHEMICYL+Y $2111.2458549 .451-19.764$

END GEOM

READ BNDS XFC=SPEC YFC=SPEC END BNDS READ PLOT TTL=!UO2 65 FULL D D INF! $P L T=Y E S P I C=M I X T U R E X U L=0 Y U L=35 Z U L=0$ $X L R=110 \quad Y L R=-35 \quad Z L R=0$

$U A X=1 \quad V D N=-1 \quad N A X=130 \quad L P I=8$

$\mathrm{NCH}=$ ! UF.W ! END PLOT

END DATA

END

\section{=CSAS25}

UO2_65_FULL_W_D

27GROUPNDFA INFHOMMEDIUM

U-234 $10.01 .540-5$

U-235 10.0 .001001

U-238 $10.08 .56-6$

H 10.0 .06661

010.0 .035354
CARBONSTEEL 21.0

END

AL $30.05 .54-4$

CA $30.05 .30-3$

C $30.06 .59-3$

H 30.0 .0133

030.0 .0457

FE $30.03 .59-4$

MG $30.03 .25-3$

SI $30.08 .43-3$

NA $30.03 .89-4$

S $30.01 .09-4$

$\mathrm{H} 2 \mathrm{O} 41.0$

END COMP

UO2 65 FULL_W_D

REA $\bar{D}$ PARAM $\bar{R} \cup \bar{N}=Y E S$ END PARAM

READ GEOM

CYLINDER 117.6219 .05 -19.05

CYLINDER $217.69919 .129-19.129$

CYLINDER $4110.9537548 .181-19.129$

CYLINDER $2111.2458549 .451-19.764$

CUBOID $3350-5050-5049.451-50.549$

END GEOM

READ BNDS XFC $=V A C$ YFC $=V A C$ END BNDS

READ PLOT TTL $=! U 0265$ FULL $W$ D!

$P L T=Y E S P I C=M I X T U R \bar{E} X \overline{U L}=-5 \overline{0}$ YŨL $=00$

$Z U L=50 X L R=50$ YLR $=00 Z Z L=-30$

$U A X=1$ WDN $=-1 \quad N A X=130 \quad L P I=8$

$\mathrm{NCH}=$ ! UFCW ! END PLOT

END DATA

END

\section{=CSAS25}

UO3_15_5_D_D

27GR̄OUPNDF 4 INFHOMMEDIUM

U-234 $10.05 .99-5$

U-235 10.0 .003878

U-238 $10.03 .33-5$

H 10.0 .059566

010.0 .037725

CARBONSTEEL 21.0

AL $30.05 .54-4$

CA $30.05 .30-3$

C $30.06 .59-3$

H 30.0 .0133

O 30.0 .0457

FE $30.03 .59-4$

MG $30.03 .25-3$

SI $30.08 .43-3$

NA $30.03 .89-4$

S $30.01 .09-4$

END

$\mathrm{H} 2 \mathrm{O} 41.0$

END COMP

UO3 155 D D

READ PATAM RUN=YES END PARAM

READ GEOM

CYLINDER $117.62-.33009-19.05$

CYLINDER $017.6219 .05-19.05$

CYIINDER $217.69919 .129-19.129$

CYLINDER $0110.9537548 .181-19.129$

CYLINDER $2111.2458549 .451-19.764$

CUBOID $3350 \quad-5050-50 \quad 49.451-50.549$

END GEOM

READ BNDS XFC $=V A C$ YFC $=V A C$ END BNDS

READ PLOT TTL=!UO3_15_5_D_DI 
$P L T=Y E S P I C=M I X T U R E X U L=50$ YUL $=00$

$Z U L=50$ XLR $=50$ YLR $=00$ ZLR $=-30$

$U A X=1$ WDN $=1 \quad N A X=130 \mathrm{LPI}=8$

$\mathrm{NCH}=$ I UFCW IEND PLOT

END DATA

END

\section{-CSAS25}

UO3 $155 \mathrm{~W}$ D

27G $\bar{R} O \bar{P} \bar{N} D \bar{F} 4$ INFHOMMEDIUM

U-234 $10.05 .7908 E-05$

U-235 $10.03 .7516 E-03$

END

U-238 $10.03 .2234 E-05$

END

H $10.05 .7627 E-02$

O $10.04 .0339 E-02$

CARBONSTEEL 21.0

AL 3 0.0 5.54-4

CA $30.05 .30-3$

C $30.06 .59-3$

H 30.0 .0133

O 30.0 .0457

FE $30.03 .59-4$

MG $30.03 .25-3$

SI $30.08 .43-3$

NA $30.03 .89-4$

S $30.01 .09-4$

$\mathrm{H} 2 \mathrm{O} 41.0$

END COMP

UO3 $155 \mathrm{~W} D$

READ PATAMM RUN=YES END PARAM READ GEOM

CYLINDER $117.62-0.330093265-19.05$

CYLINDER $017.6219 .05-19.05$

CYLINDER $217.69919 .129-19.129$

CYLINDER $4110.9537548 .181-19.129$

CYLINDER $2111.2458549 .451-19.764$

CUBOID $3350-5050-5049.451-50.549$

END GEOM

READ BNDS XFC $=V A C$ YFC $=V A C$ END BNDS

READ PLOT TTL=IUO3 $155 \mathrm{~W}$ D!

PLT $=$ YES PIC $=M I X T U R E$ XUL $=-50$ YUL $=00$

$Z U L=50 X L R=50$ YLR $=00 \quad Z L R=-30$

$U A X=1$ WDN $=-1 \quad N A X=130 \quad L P I=8$

$\mathrm{NCH}=$ ! UFCW I END PLOT

END DATA

END

\section{OXIDES IN 6-INCH CANISTERS,} 8 KG U-235

\section{=CSAS25}

UO2 C $8 \mathrm{WMO}$

27GR̄OUPNDF 4 INFHOMMEDIUM

U-234 $10.03 .6785 E-04$

U-235 $10.02 .3832 E-02$

U-238 $10.02 .0476 E-04$

O 10.0 4.8809E-02

CARBONSTEEL 21.0

AL $30.05 .54-4$

CA $30.05 .30-3$

C $30.06 .59-3$

H 30.0 .0133

O 30.0 .0457

FE $30.03 .59-4$
MG $30.03 .25-3$

END

SI $30.08 .43-3$

END

NA $30.03 .89-4$

S $30.01 .09-4$

$\mathrm{H} 2 \mathrm{O} 41.0$

END COMP

UO2 C 8 W M 0

REA $\bar{D} \overline{P A} \bar{R} A \bar{M} R \bar{U} N=Y E S$ END PARAM

READ GEOM

CYLINDER $117.62-1.4335 E+01-1.9050 E+01$

CYLINDER $417.6219 .05-19.05$

CYLINDER $217.69919 .129-19.129$

CYLINDER $4110.9537548 .181-19.129$

CYLINDER $2111.2458549 .451-19.764$

CUBOID $3350-5050-5049.451-50.549$

END GEOM

READ BNDS XFC=VAC YFC=VAC END BNDS

READ PLOT TTL $=! U O 2$ C 8 W_M_O!

PLT $=$ YES PIC $=M I X T U R \bar{X} \overline{X U}=-\overline{5} 0 \bar{Y} U L=00$

$Z U L=50 \quad X L R=50 \quad Y L R=00 \quad Z L R=-30$

$U A X=1 W D N=-1 \quad N A X=130 L P \mid=8$

$\mathrm{NCH}=$ ! UFCW $\mid$ END PLOT

END DATA

END

-CSAS25

$\mathrm{UO} 2 \mathrm{C} 8 \mathrm{~W} M$

27GR̄OU $\overline{P N D F} 4$ TNFHOMMEDIUM

U-234 $10.01 .9902 E-04$

U-235 $10.01 .2894 \mathrm{E}-02$

U-238 10.01.1078E-04

H $10.03 .0687 E-02$

O $10.04 .1751 E-02$

CARBONSTEEL 21.0

AL $30.05 .54-4$

CA $30.05 .30-3$

C $30.06 .59-3$

H 30.0 .0133

O 30.0 .0457

FE $30.03 .59-4$

MG 3 $0.03 .25-3$

SI $30.08 .43-3$

NA $30.03 .89-4$

S $30.01 .09-4$

$\mathrm{H} 2 \mathrm{O} 41.0$

END COMP

UO2 C 8 W M 4

REA $\bar{D} \overline{P A} \bar{R} A \bar{M} R \bar{R} N=Y E S$ END PARAM

READ GEOM

CYLINDER $117.62-1.0335 E+01-1.9050 E+01$

CYLINDER $417.6219 .05-19.05$

CYLINDER $217.69919 .129-19.129$

CYLINDER $4110.9537548 .181-19.129$

CYLINDER $2111.2458549 .451-19.764$

CUBOID $3350-5050-5049.451-50.549$

END GEOM

READ BNDS XFC $=V A C$ YFC $=V A C$ END BNDS

READ PLOT TTL=IUO2 C $8 \mathrm{~W} M 4 !$

PLT $=$ YES PIC $=$ MIXTURE XUL $=-\overline{50} \bar{Y} U L=00$

$Z U L=50 X L R=50$ YLR $=00 Z Z R=-30$

$U A X=1$ WDN $=-1 \quad N A X=130 \quad L P I=8$

$\mathrm{NCH}=$ ! UFCW I END PLOT

END DATA

END 
-CSAS25

UO2 C 8 W M 8

27GR̄OUPNDDF4 INFHOMMEDIUM

U-2341 $0.01 .3641 \mathrm{E}-04$

END

U-235 $10.08 .8375 E-03$

U-238 $10.07 .5929 E-05$

H 10.04 4.2067E-02

O $10.03 .9133 E-02$

CARBONSTEEL 21.0

AL $30.05 .54-4$

CA $30.05 .30-3$

C $30.06 .59-3$

H 30.0 .0133

O 30.0 .0457

FE 30.03 .59 .4

MG $30.03 .25-3$

SI $30.08 .43-3$

NA $30.03 .89-4$

S $30.01 .09-4$

$\mathrm{H} 2 \mathrm{O} 41.0$

END COMP

UO2 C 8 W $M 8$

READ PATAM RUN $=$ YES END PARAM

READ GEOM

CYLINDER $117.62-6.3350 E+00-1.9050 E+01$

CYLINDER $417.6219 .05-19.05$

CYLINDER $217.69919 .129-19.129$

CYLINDER $4110.9537548 .181-19.129$

CYLINDER $2111.2458549 .451-19.764$

CUBOID $3350-5050-5049.451-50.549$

END GEOM

READ BNDS XFC $=V A C$ YFC $=V A C$ END BNDS

READ PLOT TI=1UO2 C $8 \mathrm{~W} M 8 !$

PLT $=$ YES PIC $=$ MIXTURE $\overline{X U L}=-50$ YUL $=00$

$Z U L=50$ XLR $=50$ YLR $=00$ ZLR $=-30$

$U A X=1 W D N=-1 \quad N A X=130 \quad L P I=8$

$\mathrm{NCH}=$ ! UFCW ! END PLOT

END DATA

END

\section{$=$ CSAS25}

UO2 C 8 W M 10

27GR̄OUPNDF4 TINFHOMMEDIUM

U-234 10.0 1.1787E-04

U-235 $10.07 .6363 E-03$

END

U-238 $10.06 .5609 E-05$

END

H 10.04 .5437 E-02

O $10.03 .8358 E-02$

CARBONSTEEL 21.0

AL $30.05 .54-4$

CA $30.05 .30-3$

C $30.06 .59-3$

H 30.0 .0133

O 30.0 .0457

FE $30.03 .59-4$

MG $30.03 .25-3$

SI $30.08 .43-3$

NA $30.03 .89-4$

S $30.01 .09-4$

END

END

END

END

END

END

END

END

END

END

END

END

END

END

$\mathrm{H} 2 \mathrm{O} 41.0$

END COMP

UO2 C 8 W M 10

READ PARAM $\bar{M} \bar{U} \bar{N}=$ YES END PARAM
READ GEOM

CYLINDER $117.62-4.3350 E+00-1.9050 E+01$

CYLINDER $417.6219 .05-19.05$

CYLINDER $217.69919 .129-19.129$

CYLINDER $4110.9537548 .181-19.129$

CYLINDER $2111.2458549 .451-19.764$

CUBOID $3350-5050-5049.451-50.549$

END GEOM

READ BNDS XFC $=V A C$ YFC $=V A C$ END BNDS

READ PLOT TTL=IUO2 C 8 W_M 101

PLT $=$ YES PIC $=M I X T U R \bar{X} \bar{X} \bar{L}=-\overline{50} \bar{Y} U L=00$

$Z U L=50 X L R=50$ YLR $=00 \quad Z L R=-30$

$U A X=1$ WDN $=-1 \quad N A X=130 L P I=8$

$N C H=$ ! UFCW ! END PLOT

END DATA

END

-CSAS25

UO2 C 8 W M 15

27GROUPNDF4 INFHOMMEDIUM

U-234 $10.08 .7975 E-05$

U-235 $10.05 .6996 E-03$

U-238 $10.04 .8970 \mathrm{E}-05$

H 10.05 .0870 E-02

O $10.03 .7108 \mathrm{E}-02$

CARBONSTEEL 21.0

AL $30.05 .54-4$

CA $30.05 .30-3$

C $30.06 .59-3$

H 30.0 .0133

O 30.0 .0457

FE $30.03 .59-4$

MG $30.03 .25-3$

SI $30.08 .43-3$

NA $30.03 .89-4$

$S \quad 30.01 .09-4$

$\mathrm{H} 2 \mathrm{O} 41.0$

END COMP

UO2 C 8 W M 15

REA $\bar{D} \overline{P A} \bar{R} A \bar{M} \overline{R U N}=Y E S$ END PARAM

READ GEOM

CYLINDER 117.62 6.6499E-01 -1.9050E+01

CYLINDER $417.6219 .05-19.05$

CYLINDER $217.69919 .129-19.129$

CYLINDER $4110.9537548 .181-19.129$

CYLINDER $2111.2458549 .451-19.764$

CUBOID $3350-50 \quad 50-50 \quad 49.451-50.549$

END GEOM

READ BNDS XFC $=V A C$ YFC $-V A C$ END BNDS

READ PLOT TTL $=1 U O 2 C \& \mathrm{~W} M 15 !$

PLT $=$ YES PIC $=$ MIXTURE XUL $=-\overline{50}$ YUL $=00$

$Z U L=50 \quad X L R=50$ YLR $=00 Z Z R=-30$

$U A X=1 W D N=-1 \quad N A X=130 \quad L P I=8$

$\mathrm{NCH}=$ ! UFCW ! END PLOT

END DATA

END

$=$ CSAS25

UO2 C 8 W M 17

27GROUPNDF4 INFHOMMEDIUM

U-234 $10.08 .1099 \mathrm{E}-05$

U-235 $10.05 .2542 E-03$

END

$U-23510.05 .2542 E-03$

H $10.05 .2120 E-02$

END

END

END 


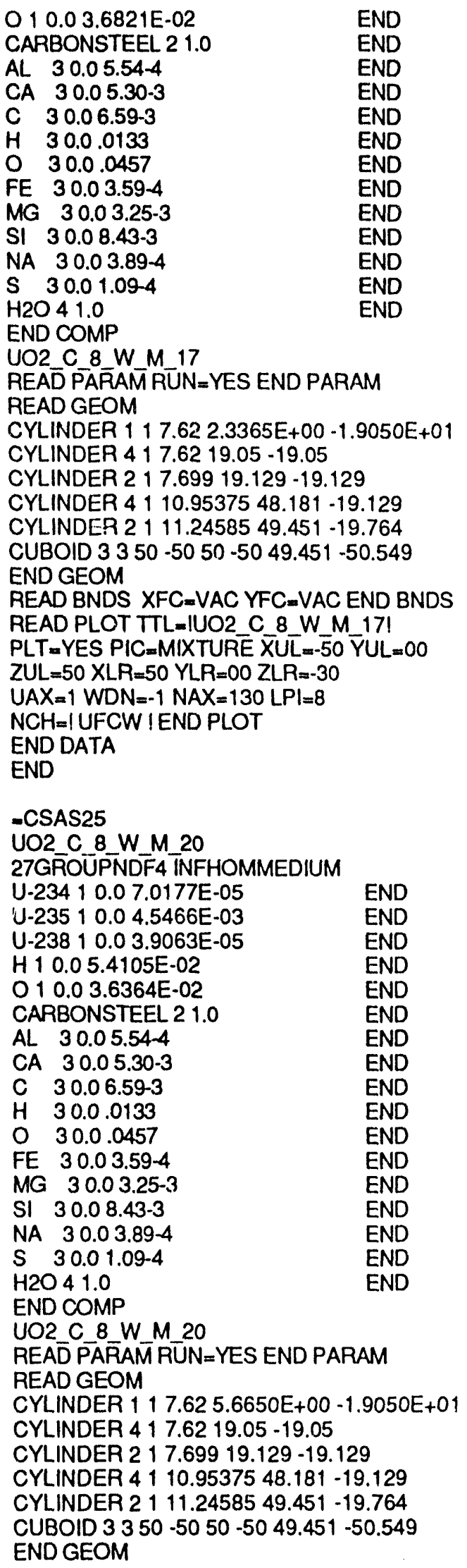

READ BNDS XFC=VAC YFC=VAC END BNDS

READ PLOT TTL=IUO2_C_8_W_M_201

PLT $=$ YES PIC $=$ MIXTURE $\bar{X} U \bar{L}=-50$ YUL $=00$

$Z U L=50 X L R=50$ YLR $=00$ ZLR $=-30$

$U A X=1$ WDN $=-1 \quad N A X=130$ LPI $=8$

NCH=IUFCW I END PLOT

END DATA

END

-CSAS25

UO2 C 8 W M 33

27GR̄OÜPNDF4 INFHOMMEDIUM

U-234 $10.04 .5523 E-05$

U-235 $10.02 .9493 E-03$

U-238 10.02 .5340 E -05

H 10.05 .8586 E-02

O $10.03 .5333 E-02$

CARBONSTEEL 21.0

$\mathrm{AL} \quad 30.05 .54-4$

CA $30.05 .30-3$

C $30.06 .59-3$

H 30.0 .0133

O 30.0 .0457

FE $30.03 .59-4$

MG $30.03 .25-3$

SI $30.08 .43-3$

NA $30.03 .89-4$

$S \quad 30.01 .09-4$

END

END

END

END

END

END

END

END

END

END

END

END

END

END

END

END

$\mathrm{H} 2 \mathrm{O} 41.0$

END COMP

UO2 C 8 W M 33

REA $\bar{D} \overline{P A} \bar{R} A \bar{M}$ RUN $=$ YES END PARAM

READ GEOM

CYLINDER $117.621 .9050 E+01-1.9050 E+01$

CYLINDER $417.6219 .05-19.05$

CYLINDER $217.69919 .129-19.129$

CYLINDER $4110.9537548 .181-19.129$

CYLINDER $2111.2458549 .451-19.764$

CUBOID $3350-5050-5049.451-50.549$

END GEOM

READ BNDS XFC $=V A C$ YFC $=V A C$ END BNDS

READ MIXT EPS $=.011$ END MIXT

READ MIXT EPS $=.011$ END MIXT

READ PLOT TTL=IUO2_C_8_W_M_33!

$P L T=Y E S P I C=M I X T U R \bar{E} \overline{X U L}=-\overline{50} \overline{Y U L}=00$

$Z U L=50$ XLR $=50$ YLR $=00 \quad Z L R=-30$

$U A X=1$ WDN $=-1 \quad N A X=130 \quad L P I=8$

$\mathrm{NCH}=$ ! UFCW ! END PLOT

END DATA

END

\section{OXIDES IN 6-INCH CANISTERS, 5 KG U-235}

$=$ CSAS25

UO2 C 5 W M O

27GROUPPNDF4 INFHOMMEDIUM

U-234 $10.03 .6785 E-04$

$\mathrm{U}-23510.02 .3832 \mathrm{E}-02$

$\mathrm{U}-23810.02 .0476 \mathrm{E}-04$

$010.04 .8809 \mathrm{E}-02$

CARBONSTEEL 21.0

AL $30.05 .54-4$

CA $30.05 .30-3$

END

END

END

END

END

END

END 


\author{
C $30.06 .59 \cdot 3$ END \\ H 30.0 .0133 END \\ O 30.0 .0457 END \\ FE $30.03 .59-4 \quad$ END \\ MG 30.03.25-3 END \\ SI $30.08 .43-3 \quad$ END \\ NA $30.03 .89-4 \quad$ END \\ $S \quad 30.01 .09-4 \quad$ END \\ $\mathrm{H} 2 \mathrm{O} 41.0$ \\ END COMP \\ UO2 C 5 W M O \\ READ PA $\bar{A} \bar{A} \bar{M}$ RŪN=YES END PARAM \\ READ GEOM \\ CYLINDER $117.62-1.6103 E+01-1.9050 E+01$ \\ CYLINDER $217.69919 .129-19.129$ \\ CYLINDER $4110.9537548 .181 \cdot 19.129$ \\ CYLINDER 2111.2458549 .451 -19.764 \\ CUBOID $3350 \quad-5050-5049.451-50.549$ \\ END GEOM \\ READ BNDS XFC.VAC YFC $=$ VAC END BNDS \\ READ PLOT TTL=IUO2_C_5_W/M OI \\ $P L T=Y E S$ PIC $=M I X T U R \bar{X} \bar{X} U \bar{L}=-\overline{50} \bar{Y} U L=00$ \\ $Z U L=50$ XLR $=50$ YLR $=00 Z Z R=-30$ \\ $U A X=1 W D N=-1 N A X=130 L P I=8$ \\ $\mathrm{NCH}=$ ! UFCW!END PLOT \\ END DATA \\ END
}

-CSAS25

UO2_C_5_W_M_2

27GR̄OUPNDF4 TNFHOMMEDIUM

U-234 $10.02 .1913 E-04$

U-235 $10.01 .4197 \mathrm{E}-02$

U-238 $10.01 .2198 E-04$

H $10.02 .7031 E-02$

O $10.04 .2592 E-02$

CARBONSTEEL 21.0

END

END

END

END

END

AL $30.05 .54-4$

CA $30.05 .30-3$

C $30.06 .59-3$

H 30.0 .0133

O 30.0 .0457

FE $30.03 .59-4$

MG $30.03 .25-3$

SI $30.08 .43-3$

NA $30.03 .89-4$

END

END

END

END

END

END

END

END

END

END

S $30.01 .09-4$

END

END COMP

UO2 C 5 W M 2

REA $\bar{D} \overline{P A} \bar{R} A \bar{M}$ RŨN $=$ YES END PARAM

READ GEOM

CYLINDER $117.62-1.4103 E+01-1.9050 E+01$

CYLINDER $417.6219 .05-19.05$

CYLINDER $217.69919 .129-19.129$

CYLINDER $4110.9537548 .181-19.129$

CYLINDER $2111.2458549 .451-19.764$

CUBOID $3350-50 \quad 50-5049.451-50.549$

END GEOM

READ BNDS XFC=VAC YFC=VAC END BNDS

READ PLOT TTL=!UO2 C $5 \mathrm{~W} M 2$ M

PLT $=$ YES PIC $=$ MIXTURE $\bar{X} U \bar{L}=-\overline{50} \bar{Y} U L=00$

$Z U L=50$ XLR $=50$ YLR $=00 Z Z R=-30$

$U A X=1 W D N=-1 N A X=130 L P I=8$
NCH=I UFCWIEND PLOT

END DATA

END

-CSAS25

UO2 C 5 W M M 10

27GR̄OUPNDF 4 TNFHOMMEDIUM

$U-23410.0$ 8.3728E-05

U-235 $10.05 .4245 E-03$

U-238 10.0 4.6606E-05

H $10.05 .1642 E-02$

O $10.03 .6931 \mathrm{E}-02$

CARBONSTEEL 21.0

AL $30.05 .54-4$

CA $30.05 .30-3$

C $30.06 .59-3$

H $\quad 30.0 .0133$

O 30.0 .0457

FE $30.03 .59-4$

MG $30.03 .25-3$

SI $30.08 .43-3$

NA $30.03 .89-4$

S $30.01 .09-4$

$\mathrm{H} 2 \mathrm{O} 41.0$

END COMP

UO2 C 5 W M 10

READ PARAM $\bar{M} \bar{U} N=$ YES END PARAM

READ GEOM

CYLINDER $117.62-6.1031 E+00-1.9050 E+01$

CYLINDER $417.6219 .05-19.05$

CYLINDER $217.69919 .129-19.129$

CYLINDER $4110.9537548 .181-19.129$

CYLINDER $2111.2458549 .451-19.764$

CUBOID $3350-5050-5049.451-50.549$

END GEOM

READ BNDS XFC=VAC YFC=VAC END BNDS

READ PLOT TTL $=$ !UO2 C 5 W M $10 !$

PLT $=$ YES PIC $=M I X T U R \bar{X} \bar{X} \bar{L}=-50$ YUL $=00$

$Z U L=50 X L R=50$ YLR $=00 \quad Z L R=-30$

$U A X=1 W D N=-1 \quad N A X=130 \quad L P I=8$

$\mathrm{NCH}=$ ! UFCWIEND PLOT

END DATA

END

-CSAS25

UO2_C 5 W_M 18.4

27GR̄OUTNDDF4 INFHOMMEDIUM

U-234 10.0 5.0687E-05

U-235 $10.03 .2839 E-03$

U-238 $10.02 .8214 E-05$

H 1 0.0 5.7647E-02

O 1 0.0 3.5549E-02

CARBONSTEEL 21.0

AL $30.05 .54-4$

CA $30.05 .30-3$

C $30.06 .59-3$

H 30.0 .0133

O 30.0 .0457

FE $30.03 .59-4$

MG $30.03 .25-3$

SI $30.08 .43-3$

NA $30.03 .89-4$

S $30.01 .09-4$

$\mathrm{H} 2 \mathrm{O} 41.0$ 
END COMP

UO2 C 5 W M 18.4

REA $\bar{D} \overline{P A} \overline{R A M}$ RŪ $\mathrm{N}=$ YES END PARAM

READ GEOM

CYLINDER $117.622 .3365 E+00-1.9050 E+01$

CYLINDER $417.6219 .05-19.05$

CYLINDER $217.69919 .129-19.129$

CYLINDER $4110.9537548 .181-19.129$

CYLINDER 2111.2458549 .451 -19.764

CUBOID $3350-5050-5049.451-50.549$

END GEOM

READ BNDS XFC $=V A C$ YFC $=V A C$ END BNDS

READ PLOT $T L=\mid U O 2$ C $5 \mathrm{~W} M 18.41$

PLT $=$ YES PIC $=M I X T U R \bar{X} \bar{X} \bar{L}=-50$ YUL $=00$

$Z U L=50 X L R=50$ YLR $=00 Z L R=-30$

$U A X=1$ WDN $=-1 \quad N A X=130 \quad L P I=8$

$\mathrm{NCH}=$ ! UFCWIEND PLOT

END DATA

END

\section{$=$ CSAS25}

UO2 C 5 W M 20

27GR̄OUPNDF4 INFHOMMEDIUM

U-234 $10.04 .7240 E-05$

U-235 $10.03 .0606 E-03$

U-238 $10.02 .6295 \mathrm{E}-05$

H $10.05 .8274 E-02$

O $10.03 .5405 E-02$

CARBONSTEEL 21.0

AL $30.05 .54-4$

CA $30.05 .30-3$

C $30.06 .59-3$

H 30.0 .0133

O 30.0 .0457

FE $30.03 .59-4$

MG $30.03 .25-3$

SI $30.08 .43-3$

NA $30.03 .89-4$

S $30.01 .09-4$

$\mathrm{H} 2 \mathrm{O} 41.0$

END COMP

UO2 C 5 W M 20

REA $\bar{D} \overline{P A} \bar{R} A \bar{M}$ RU $\bar{N}=Y E S$ END PARAM

READ GEOM

CYLINDER $117.623 .8969 E+00-1.9050 E+01$

CYLINDER $417.6219 .05-19.05$

CYLINDER $217.69919 .129-19.129$

CYLINDER $4110.9537548 .181-19.129$

CYLINDER $2111.2458549 .451-19.764$

CUBOID $3350-5050-5049.451-50.549$

END GEOM

READ BNDS XFC $=V A C$ YFC $=V A C$ END BNDS

READ PLOT TTL $=! U O 2 C 5 W M 20$ !

PLT $=$ YES PIC $=M I X T I S R E \overline{X U L}=-50$ YUL $=00$

$Z U L=50 X L R=50$ YLR $=00 Z L R=-30$

$U A X=1 W D N=-1 \quad N A X=130 L P I=8$

$\mathrm{NCH}=$ ! UFCW!END PLOT

END DATA

END

\section{-CSAS25}

UO2_C_5_W_M_35.2

27GROUUPNDF4 INFHOMMEDIUM

U-234 $10.02 .8452 E-05$

END
U-235 $10.01 .8433 E-03$

END

U-238 $10.01 .5837 E-05$

H $10.06 .1689 E-02$

O 10.03 .4620 E-02

CARBONSTEEL 21.0

AL $30.05 .54-4$

CA $30.05 .30-3$

C $30.06 .59-3$

H $\quad 30.0 .0133$

0 2 0.0457

FE $30.03 .59-4$

MG $30.03 .25-3$

SI $30.08 .43-3$

NA $30.03 .89-4$

S $30.01 .09-4$

END

END

END

END

END

END

END

END

END

END

END

END

END

END

$\mathrm{H} 2 \mathrm{O} 41.0$

END COMP

UO2 C 5 W M 35.2

REA $\bar{D} \overline{P A} \bar{R} A \bar{M} R \bar{U} N=Y E S$ END PARAM

READ GEOM

CYLINDER $117.621 .9050 E+01-1.9050 E+01$

CYLINDER $217.69919 .129-19.129$

CYLINDER $4110.9537548 .181-19.129$

CYLINDER $2111.2458549 .451-19.764$

CUBOID $3350-5050-5049.451-50.549$

END GEOM

READ BNDS $X F C=V A C$ YFC $=V A C$ END BNDS

READ PLOT TTL=IUO2 C $5 \mathrm{~W} M 35.2$ !

$P L T=Y E S$ PIC $=M I X T U R E \overline{X U L}=-50$ YUL $=00$

$Z U L=50 X L R=50$ YLR $=00$ ZLR $=-30$

$U A X=1$ WDN $=-1 \quad N A X=130$ LPI $=8$

$\mathrm{NCH}=$ ! UFCWIEND PLOT

END DATA

END

\section{OXIDES IN 6-INCH CANISTERS, 2 KG U-235}

=CSAS25

UO2 C 2 W M $O$

27GROUTPNDF4 INFHOMMEDIUM

U-234 $10.03 .6785 E-04$

U-235 $10.02 .3832 E-02$

U-238 $10.02 .0476 E-04$

O $10.04 .8809 E-02$

CARBONSTEEL 21.0

AL $30.05 .54-4$

CA $30.05 .30-3$

C $30.06 .59-3$

H 30.0 .0133

O 30.0 .0457

FE $30.03 .59-4$

$M G \quad 30.03 .25-3$

SI $30.08 .43-3$

NA $30.03 .89-4$

$S \quad 30.01 .09-4$

$\mathrm{H} 2 \mathrm{O} 41.0$

END COMP

UO2 C $2 W M O$

READ PADAM $\bar{M} \bar{R} \bar{N}=Y E S$ END PARAM

READ GEOM

CYLINDER $117.62-1.7871 E+01-1.9050 E+01$

CYLINDER $217.69919 .129-19.129$

CYLINDER $4110.9537548 .181-19.129$ 
CYLINDER $2111.2458549 .451-19.764$ CUBOID $3350-5050-5049.451$-50.549 END GEOM

READ BNDS XFC=VAC YFC-VAC END BNDS READ PLOT TIL=IUO2_C_2W_M_O!

PLT=YES PIC-MIXTURE XU $\bar{L}=-50$ YUL $=00$

ZUL $=50$ XLR-50 YLR-00 ZLR=-30

$U A X=1$ WDN $=1$ NAX $=130 \quad \mathrm{LPI}=8$

$\mathrm{NCH}=$ I UFCW I END PLOT

END DATA

END

\section{-CSAS25}

UO2 C 2 W M 2

27GR̄OUPNDF4 INFHOMMEDIUM

U.234 $10.01 .3641 \mathrm{E}-04$

U-235 $10.08 .8375 E-03$

U.238 $10.07 .5929 E-05$

H $10.04 .2067 \mathrm{E}-02$

O $10.03 .9133 E-02$

CARBONSTEEL 21.0

AL $30.05 .54-4$

CA $30.05 .30-3$

C 30.06 .593

H 30.0 .0133

O 30.0 .0457

FE $30.03 .59-4$

MG $30.03 .25-3$

SI $30.08 .43-3$

NA $30.03 .89-4$

S $30.01 .09-4$

$\mathrm{H} 2 \mathrm{O} 41.0$

END COMP

UO2_C_ 2 W M 2

REA $\bar{D} \overline{P A} \bar{A} A \bar{M} R \bar{U} N=Y E S$ END PARAM

READ GEOM

CYLINDER $117.62-1.5871 E+01-1.9050 E+01$

CYLINDER $417.6219 .05-19.05$

CYLINDER $217.69919 .129-19.129$

CYLINDER $4110.9537548 .181-19.129$

CYLINDER $2111.2458549 .451-19.764$

CUBOID $3350-5050-50 \quad 49.451-50.549$

END GEOM

READ BNDS XFC=VAC YFC=VAC END BNDS

READ PLOT TTL=IUO2_C $2 W M 21$

PLT $=$ YES PIC $=M I X T U R E \overline{X U}=-50$ YUL $=00$

$Z U L=50$ XLR $=50$ YLR $=00$ ZLR $=30$

$U A X=1$ WDN $=-1$ NAX $=130$ LPI $=8$

$\mathrm{NCH}=\mid$ UFCW $\mid$ END PLOT

END DATA

END

=CSAS25

UO2 C 2 W M 8

27GROUTPNDF4 INFHOMMEDIUM

U.234 $10.04 .7240 \mathrm{E}-05$

$U-235+0.03 .0606 E-03$

U-238 $10.02 .6295 E-05$

H $10.05 .8274 E-02$

O $10.03 .5405 E-02$

CARBONSTEEL 21.0

AL $30.05 .54-4$

CA $30.05 .30-3$

C $30.06 .59-3$
H $\quad 30.0 .0133$

END

O 30.0 .0457

FE $30.03 .59-4$

MG $30.03 .25-3$

SI $30.08 .43-3$

NA $30.03 .89-4$

S $30.01 .09-4$

$\mathrm{H} 2 \mathrm{O} 41.0$

END COMP

UO2 C 2 W M 8

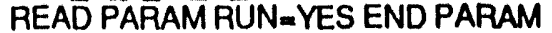

READ GEOM

CYLINDER $117.62-9.8713 E+00-1.9050 E+01$

CYLINDER $417.6219 .05-19.05$

CYLINDER $217.69919 .129-19.129$

CYLINDER $4110.9537548 .181-19.129$

CYLINDER $2111.2458549 .451-19.764$

CUBOID $3350-5050-50 \quad 49.451$ - 50.549

END GEOM

READ BNDS XFC $=V A C$ YFC $=V A C$ END BNDS

READ PLOT TTL=IUO2_C_2_W_M_8I

$P L T=Y E S$ PIC $=$ MIXTURE $\bar{X} \bar{L}=-\overline{50} \bar{Y} U L=00$

$Z U L=50$ XLR $=50$ YLR $=00$ ZLR -30

$U A X=1$ WDN $=-1 \quad N A X=130$ LPI $=8$

$\mathrm{NCH}=$ !UFCW ! END PLOT

END DATA

END

-CSAS25

UO2_C_2_W_M_10

27GROUUTNDF4 TNFHOMMEDIUM

U-234 $10.03 .8788 E-05$

U-235 $10.02 .5130 E-03$

U-238 $10.02 .1591 E-05$

H $10.05 .9810 E-02$

O $10.03 .5052 E-02$

CARBONSTEEL 21.0

AL $30.05 .54-4$

CA $30.05 .30-3$

C $30.06 .59-3$

H 30.0 .0133

O $\quad 30.0 .0457$

FE $30.03 .59-4$

MG $30.03 .25-3$

SI $30.08 .43-3$

NA $30.03 .89-4$

S $30.01 .09-4$

$\mathrm{H} 2 \mathrm{O} 41.0$

END COMP

UO2_C 2 W M 10

REA $\bar{D} \overline{P A} \bar{R} A \bar{M} R \bar{R} N=$ YES END PARAM

READ GEOM

CYLINDER $117.62-7.8713 E+00-1.2050 E+01$

CYLINDER $417.6219 .05-19.05$

CYLINDER $217.69919 .129-19.129$

CYLINDER $4110.9537548 .181-19.129$

CYLINDER $2111.2458549 .451-19.764$

CUBOID $3350-50 \quad 50-50 \quad 49.451 \quad-50.549$

END GEOM

READ BNDS XFC-VAC YFC-VAC END BNDS

READ PLOT TTL=!UO2_C_2_W_M_101

PLT $=$ YES PIC=MIXTURE $\overline{X U} \bar{L}=-50$ Y $U L=00$

$Z U L=50$ XLR $=50$ YLR $=00$ ZLR $=-30$

$U A X=1$ WDN $=-1$ NAX $=130 \mathrm{LPI}=8$ 
NCH=! UFCW I END PLOT

END DATA

END

$=$ CSAS25

UO2 C 2 W M 20

27GR̄OUTNDDF4 INFHOMMEDIUM

U-234 10.02 2.0474E-05

U-235 $10.01 .3264 E-03$

END

U-238 $10.01 .1396 E-05$

END

H $10.06 .3139 E-02$

O $10.03 .42 .86 E-02$

CARBONSTEEL 21.0

AL $30.05 .54-4$

CA $30.05 .30-3$

C $30.06 .59-3$

H 30.0 .0133

O 30.0 .0457

FE $30.03 .59-4$

MG $30.03 .25-3$

SI $30.08 .43-3$

NA $30.03 .89-4$

S $30.01 .09-4$

$\mathrm{H} 2 \mathrm{O} 41.0$

END COMP

UO2 C 2 W M 20

READ PA $\bar{R} A \bar{M}$ RUUN $=$ YES END PARAM

READ GEOM

CYLINDER $117.622 .1287 E+00-1.9050 E+01$

CYLINDER $417.6219 .05-19.05$

CYLINDER $217.69919 .129-19.129$

CYLINDER $4110.9537548 .181-19.129$

CYLINDER $2111.2458549 .451-19.764$

CUBOID $3350-5050-5049.451-50.549$

END GEOM

READ BNDS XFC $=V A C$ YFC $=V A C$ END BNDS

READ PLOT TTL=!UO2 C $2 \mathrm{~W} M 20$ !

$P L T=Y E S$ PIC $=M I X T U R E \bar{X} U \bar{L}=-50 \bar{Y} U L=00$

$Z U L=50 \times L R=50$ YLR $=00$ ZLR $=-30$

$U A X=1$ WDN=-1 NAX $=130 \mathrm{LPI}=8$

$\mathrm{NCH}_{2}$ ! UFCW ! END PLOT

END DATA

END

\section{=CSAS25}

UO2 C 2 W M 36.9

27GROUTPNDF4 4 INFHOMMEDIUM

U-234 $10.01 .1381 E-05$

U-235 $10.07 .3732 E-04$

U-238 $10.06 .3349 E-06$

H $10.06 .4791 \mathrm{E}-02$

O 1 0.0 3.3906E-02

CARBONSTEEL 21.0

AL $30.05 .54-4$

CA $30.05 .30-3$

C $30.06 .59-3$

H 30.0 .0133

O $\quad 30.0 .0457$

FE $30.03 .59-4$

MG $30.03 .25-3$

SI $30.08 .43-3$

NA $30.03 .89-4$

S $30.01 .09-4$

$\mathrm{H} 2 \mathrm{O} 41.0$
END COMP

UO2 C 2 W M 36.9

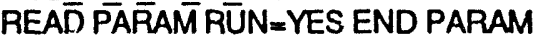

READ GEOM

CYLINDER 117.62 1.9050E+01 -1.9050E^n

CYLINDER $217.69919 .129-19.129$

CYLINDER $4110.9537548 .181-19.129$

CYLINDER $2111.2458549 .451-19.764$

CUBOID $3350-5050-50 \quad 49.451-50.549$

END GEOM

READ BNDS XFC $=V A C$ YFC $=V A C$ END BNDS READ PLOT TTL=!UO2 C 2 W M 36.9 !

PLT $=$ YES PIC $=M \mid X T U R \bar{X} \overline{X U} \bar{L}=-\overline{50} \bar{Y} U L=00$

$Z U L=50 X L R=50$ YLR $=00$ ZLR $=-30$

$U A X=1 \quad W D N=-1 \quad N A X=130 \quad L P I=8$

$\mathrm{NCH}=$ ! UFCW ! END PLOT

END DATA

END

OXIDES IN 6-INCH CANISTERS, 8 KG U-235, CONCRETE H VARIATION

=CSAS 25

UO2 108 D D INF OH

27GROUPNDF 4 INFHOMMEDIUM

U.234 $10.08 .1100-5$

U-235 $10.05 .254-3$

U-238 $10.04 .51-5$

$\mathrm{H} \cap 5.3804-2$

C 7663-2

( EEL 21.0

A. $\quad .54-4$

CA $\cup 5.30-3$

C $30.06 .59-3$

O 30.0 .0457

FE $30.03 .59-4$

MG $30.03 .25-3$

SI $30.08 .43-3$

NA $30.03 .89-4$

S $30.01 .09-4$

$\mathrm{H} 2 \mathrm{O} 41.0$

END COMP

UO2 108 D D INF OH

READ PĀRAM $R \bar{U} \bar{N}=\bar{Y} E S$ END PARAM

READ GEOM

UNIT 1

ZHEMICYL $+X 117.622 .3364508-1.9050 E+01$

ZHEMICYL+X $017.622 P 19.05$

ZHEMICYL+X 217.699 2P19.129

ZHEMICYL+X $0110.9537548 .181-19.129$

ZHEMICYL+X $21 \quad 11.2458549 .451-19.764$

CUBOID $31105.5675030 .48-30.4849 .451$

$-35.004$

HOLE 2105.567500

HOLE 352.7837530 .480

HOLE $452.78375-30.480$

UNIT 2

ZHEMICYL-X $117.622 .3364508-1.9050 E+01$

ZHEMICYL-X $017.622 P 19.05$

ZHEMICYL-X 217.699 2P 19.129

ZHEMICYL-X $01 \quad 10.9537548 .181-19.129$

ZHEMICYL-X $X 2111.2458549 .451-19.764$

UNIT 3 
ZHEMICYL-Y 117.622 .3364508 -1.9050E+01 ZHEMICYL-Y $017.622 P 19.05$

ZHEMICYL-Y 217.699 2P19.129

ZHEMICYL-Y $0110.9537548 .181-19.129$

ZHEMICYL-Y $2111.2458549 .451-19.764$

UNIT 4

ZHEMICYL+Y $117.622 .3364508-1.9050 E+01$

ZHEMICYL+Y $017.622 P 19.05$

ZHEMICYL+Y $217.6992 P 19.129$

ZHEMICYL+YO $110.9537548 .181-19.129$

ZHEMICYL+Y $21 \quad 11.2458549 .451-19.764$

END GEOM

READ BNDS XFC $=$ SPEC YFC $=S P E C$ END BNDS

READ PLOT TTL=!UO2 $108 \mathrm{D} D$ INF OH!

PLT $=Y E S$ PIC $=$ MIXTURE $X \bar{U} \bar{L}=0 \bar{Y} \bar{U}=3 \bar{Z} \mathrm{ZUL}=0$

$X I R=110 \quad Y L R=-35 \quad Z L R=0$

$U A X=1$ VDN $=-1 \quad N A X=130 \quad L P I=8$

$\mathrm{NCH}=$ ! UF.W ! END PLOT

END DATA

END

\section{$=$ CSAS25}

UO2 10_8_D_D_INF_.2H

27GR̄OUPNDFA INFHÖMMEDIUM

U-2341 $0.08 .1100-5$

U-235 $10.05 .254-3$

U-238 $10.04 .51-5$

H $10.05 .3804-2$

O $10.03 .7663-2$

CARBONSTEEL 21.0

AL $30.05 .54-4$

CA $30.05 .30-3$

C $30.06 .59-3$

H 30.0 .00266

O $\quad 30.0 .0457$

FE $30.03 .59-4$

MG $30.03 .25-3$

SI $30.08 .43-3$

NA 30.03 .89 .4

S $30.01 .09-4$

$\mathrm{H} 2 \mathrm{O} 41.0$

END COMP

UO2_10_8_D_D_INF_.2H

READ PARAM $\bar{D}$ RÜN $=\bar{Y} E S$ END PARAM

\section{READ GEOM}

UNIT 1

ZHEMICYL+X $117.622 .3364508-1.9050 E+01$ $Z H E M I C Y L+X 017.622 P 19.05$

ZHEMICYL+X $217.6992 P 19.129$

ZHEMICYL+X $0110.9537548 .181-19.129$

ZHEMICYL+X $2111.2458549 .451-19.764$ CUBOID $31105.5675030 .48-30.4849 .451$ $-35.004$

HOLE 2105.567500

HOLE 352.7837530 .480

HOLE $452.78375-30.480$

UNIT 2

ZHEMICYL-X $117.622 .3364508-1.9050 E+01$

ZHEMICYL-X 017.622 P 19.05

ZHEMICYL-X 217.699 2P19.129

ZHEMICYL-X $0110.9537548 .181-19.129$

ZHEMICYL-X $2111.2458549 .451-19.764$

UNIT 3

- ZHEMICYL-Y 117.622 .3364508 -1.9050E+01
ZHEMICYL-Y 017.62 2P19.05

ZHEMICYL-Y 217.699 2P19.129

ZHEMICYL-Y $0110.9537548 .181-19.129$

ZHEMICYL-Y $2111.2458549 .451-19.764$

UNIT 4

ZHEMICYL+Y $117.622 .3364508-1.9050 E+01$

ZHEMICYL+Y 017.62 2P19.05

ZHEMICYL+Y $217.6992 P 19.129$

ZHEMICYL+YO $110.9537548 .181-19.129$

ZHEMICYL+Y $2111.2458549 .451-19.764$

END GEOM

READ BNDS XFC=SPEC YFC $=S P E C$ END BNDS

READ PLOT TTL=!UO2_10_8_D_D_INF_.2H!

PLT $=Y E S$ PIC $=$ MIXTURE $X \bar{U} L=0 \bar{Y} \bar{U} L=35 \mathrm{ZUL}=0$

$X L R=110$ YLR $=-35$ ZLR $=0$

$\cup A X=1$ VDN $=-1 \quad$ NAX $=130 \quad L P I=8$

$\mathrm{NCH}=$ !UF.W I END PLOT

END DATA

END

\section{$=$ CSAS25}

UO2 108 D D INF .5H

27GR̄OUPNDDF4 INFHOMMEDIUM

U-234 $10.08 .1100-5$

U-235 $10.05 .254-3$

U-238 $10.04 .51-5$

H $10.05 .3804-2$

O 1 $10.03 .7663-2$

CARBONSTEEL 21.0

AL $30.05 .54-4$

CA $30.05 .30-3$

C $30.06 .59-3$

H 30.0 .0066667

O 30.0 .0457

FE $30.03 .59-4$

MG $30.03 .25-3$

SI $30.08 .43-3$

NA $30.03 .89-4$

S $30.01 .09-4$

$\mathrm{H} 2 \mathrm{O} 41.0$

END COMP

UO2 10_8_D_D INF.5H

READ PARAM RU $N=\bar{Y} E S$ END PARAM

READ GEOM

UNIT 1

ZHEMICYL+X $117.622 .3364508-1.9050 E+01$

ZHEMICYL+X $017.622 \mathrm{P} 19.05$

ZHEMICYL+X $217.6992 P 19.129$

ZHEMICYL+X $0110.9537548 .181-19.129$

ZHEMICYL+X $21 \quad 11.2458549 .451-19.764$

CUBOID $31105.5675030 .48-30.4849 .451$

$-35.004$

HOLE 2105.567500

HOLE 352.7837530 .480

HOLE $452.78375-30.480$

UNIT 2

ZHEMICYL-X $117.622 .3364508-1.9050 E+01$

ZHEMICYL-X $017.622 P 19.05$

ZHEMICYL-X 217.699 2P19.129

ZHEMICYL-X $0110.9537548 .181-19.129$

ZHEMICYL-X $2111.2458549 .451-19.764$

UNIT 3

ZHEMICYL-Y $117.622 .3364508-1.9050 E+01$ ZHEMICYL-YO 17.62 2P19.05 
ZHEMICYL-Y 217.699 2P19.129

ZHEMICYL-Y $0110.9537548 .181-19.129$

ZHEMICYL-Y $21 \quad 11.2458549 .451$-19.764

UNIT 4

ZHEMICYL+Y $117.622 .3364508-1.9050 E+01$

ZHEMICYL+Y 017.62 2P19.05

ZHEMICYL+Y $217.6992 P 19.129$

ZHEMICYL+Y $0110.9537548 .181-19.129$

ZHEMICYL+Y $2111.2458549 .451-19.764$

END GEOM

READ BNDS $X F C=S P E C$ YFC $=S P E C$ END BNDS

READ PLOT TTL $=\mid U O 2$ 2_10_8_D_D_INF_.5H!

PLT $=$ YES PIC $=M I X T U R \bar{E} X \bar{U} L=0 \overline{Y U L}=3 \overline{5} \mathrm{ZUL}=0$

$X L R=110$ YLR $=-35$ ZLR $=0$

$U A X=1$ VDN $=-1 \quad N A X=130 L P \mid=8$

$\mathrm{NCH}=$ ! UF.W ! END PLOT

END DATA

END

=CSAS25

UO2 10 8 D D_INF

27GROUP:IDF4 INFHOMMEDIUM

U-2341 $0.08 .1100-5$

U-235 $10.05 .254-3$

U.238 $10.04 .51-5$

H $10.05 .3804-2$

O $10.03 .7663-2$

CARBONSTEEL 21.0

AL $30.05 .54-4$

CA $30.05 .30-3$

C $30.06 .59-3$

H 30.0 .0133

O 30.0 .0457

FE $30.03 .59-4$

MG $30.03 .25-3$

SI $30.08 .43-3$

NA $30.03 .89-4$

S $30.01 .09-4$

$\mathrm{H} 2 \mathrm{O} 41.0$

END COMP

UO2 108 D D INF

READ PATAMM $\overline{R U} N=Y=S$ END PARAM

READ GEOM

UNIT 1

ZHEMICYL+X $117.622 .3364508-1.9050 E+01$

ZHEMICYL+X $017.622 P 19.05$

ZHEMICYL+X $217.6992 P 19.129$

$Z H E M I C Y L+X 0110.9537548 .181-19.129$

ZHEMIC' ' $+X 2111.2458549 .451-19.764$

CUBOID $31105.5675030 .48-30.4849 .451$

$-35.004$

HOLE 2105.567500

HOLE 352.7837530 .480

HOLE $452.78375-30.480$

UNIT 2

ZHEMICYL-X $117.622 .3364508-1.9050 E+01$

ZHEMICYL-X 017.62 2P19.05

ZHEMICYL-X $217.6992 P 19.129$

ZHEMICYL-X $0110.9537548 .181-19.123$

ZHEMICYL-X 2111.2458549 .451 -19.764

UNIT 3

ZHEMICYL-Y 117.622 .3364508 -1.9050E+01

ZHEMICYL-Y 017.62 2P19.05

ZHEMICYL-Y 217.699 2P19.129
ZHEMICYL-Y $01 \quad 10.9537548 .181-19.129$

ZHEMICYL-Y $21 \quad 11.2458549 .451-19.764$

UNIT 4

ZHEMICYL+Y $117.622 .3364508-1.9050 E+01$

ZHEMICYL+Y $017.622 P 19.05$

ZHEMICYL+Y 217.699 2P19.129

ZHEMICYL+Y $0110.9537548 .181-19.129$

ZHEMICYL+Y $2111.2458549 .451-19.764$

END GEOM

READ BNDS XFC=SPEC YFC=SPEC END BNDS

READ PLOT TTL $=$ !UO2 $108 \mathrm{D} D$ INFI

$P L T=Y E S P I C=M I X T U R \bar{E} X \bar{U} L=0 \bar{Y} \bar{U}=35 \quad Z U L=0$

$X L R=110 Y L R=-35 Z L R=0$

$U A X=1$ VDN $=-1 \quad N A X=130 \quad L P I=8$

$\mathrm{NCH}=$ ! UF.W ! END PLOT

END DATA

END

=CSAS 25

UO2_10_8_D_D_INF_2H

27GR̄OUPNDDF4 INFHOMMEDIUM

U-234 $10.08 .1100-5$

U-235 $10.05 .254-3$

U.238 $10.04 .51-5$

H $10.05 .3804-2$

O $10.03 .7663-2$

CARBONSTEEL 21.0

AL. $30.05 .54-4$

CA $30.05 .30-3$

C $30.06 .59-3$

H 30.0 .0266

O 30.0 .0457

FE $30.03 .59-4$

MG $30.03 .25-3$

SI $30.08 .43-3$

NA $30.03 .89-4$

S $30.01 .09-4$

$\mathrm{H} 2 \mathrm{O} 41.0$

END COMP

UO2 108 D D INF 2H

READ PATRAMM RUN $=\bar{Y} E S$ END PARAM

READ GEOM

UNIT 1

ZHEMICYL+X $117.622 .3364508-1.9050 E+01$

ZHEMICYL+X $017.622 \mathrm{P} 19.05$

ZHEMICYL+X $217.6992 P 19.129$

ZHEMICYL+X $0110.9537548 .181-19.129$

ZHEMICYL+X $21 \quad 11.2458549 .451-19.764$

CUBOID $31105.5675030 .48-30.4849 .451$

$-35.004$

HOLE 2105.567500

HOLE 352.7837530 .480

HOLE $452.78375-30.480$

UNIT 2

ZHEMICYL-X $117.622 .3364508-1.9050 E+01$

ZHEMICYL-X 017.622 P 19.05

ZHEMICYL-X 217.699 2P19.129

ZHEMICYL-X $0110.9537548 .181-19.129$

ZHEMICYL-X $2111.2458549 .451-19.764$

UNIT 3

ZHEMICYL-Y 117.622 .3364508 -1.9050E+01

ZHEMICYL-Y 017.62 2P19.05

ZHEMICYL-Y 217.699 2P19.129

ZHEMICYL-Y O $1 \quad 10.9537548 .181-19.129$ 
ZHEMICYL-Y $21 \quad 11.2458549 .451-19.764$

UNIT 4

ZHEMICYL+Y $117.622 .3364508-1.9050 E+01$

ZHEMICYL+Y $017.622 P 19.05$

ZHEMICYL+Y 217.699 2P19.129

ZHEMICYL+YO $110.9537548 .181-19.129$

$Z$ ZHEMICYL+Y $21 \quad 11.2458549 .451-19.764$

END GEOM

READ BNDS $X F C=S P E C$ YFC $=S P E C$ END BNDS

READ PLOT TTL=IUO2 $108 \mathrm{D} D$ INF $2 \mathrm{H}$ !

$P L T=Y E S$ PIC $=M I X T U R E X \bar{U} L=0$ Y $\overline{U L}=35 \quad Z U L=0$

$X L R=110$ YLR $=-35$ ZLR $=0$

$U A X=1$ VDN $=-1 \quad N A X=130$ LPI $=8$

$\mathrm{NCH}=!$ UF.W IEND PLOT

END DATA

OXIDES IN 6-INCH CANISTERS, $8 \mathrm{KG} \mathrm{U}-235, \mathrm{UO}_{2}$ DENSITY VARIATION

\section{$=$ CSAS25}

UO2_C_8_D_D_INF_0

27GR

U-234 $10.03 .6785 E-04$

U-235 $10.02 .3832 E-02$

U-238 10.02 .0476 E-04

$010.04 .8809 E-02$

CARBONSTEEL 21.0

AL $30.05 .54-4$

CA $30.05 .30-3$

C $30.06 .59-3$

H 30.0 .0133

O 30.0 .0457

FE $30.03 .59-4$

MG $30.03 .25-3$

SI $30.08 .43-3$

NA $30.03 .89-4$

$S \quad 30.01 .09-4$

$\mathrm{H} 2 \mathrm{O} 41.0$

END COMP

UO2 C 8 D D INF 0

READ $\overline{P A} \bar{R} A \bar{M} \bar{R} U N=\bar{Y}$ YES END PARAM

READ GEOM

UNIT 1

ZHEMICYL+X $117.62-1.4335 E+01-19.05$

ZHEMICYL+X $017.622 P 19.05$

ZHEMICYL+X $217.6992 P 19.129$

ZHEMICYL+X $0110.9537548 .181-19.129$

ZHEMICYL+X $21 \quad 11.2458549 .451-19.764$

CUBOID $31105.5675030 .48-30.4849 .451$

$-35.004$

HOLE 2105.567500

HOLE 352.7837530 .480

HOLE $452.78375-30.480$

UNIT 2

ZHEMICYL.X 117.62 -1.4335E+01 19.05

ZHEMICYL-X 017.62 2P19.05

ZHEMICYL-X $217.6992 \mathrm{P} 19.129$

ZHEMICYL-X $0110.9537548 .181-19.129$

ZHEMICYL-X 2111.2458549 .451 -19.764

UNIT 3

ZHEMICYL-Y 117.62 -1.4335E+01 -19.05

ZHEMICYL-Y 017.62 2P19.05

ZHEMICYL-Y 217.699 2P19.129
ZHEMICYL-Y $0110.9537548 .181-19.129$

ZHEMICYL-Y $21 \quad 11.2458549 .451-19.764$

UNIT 4

ZHEMICYL+Y $117.62-1.4335 E+01-19.05$

ZHEMICYL+Y 017.62 2P19.05

ZHEMICYL+Y $217.6992 P 19.129$

ZHEMICYL+Y $0110.9537548 .181-19.129$

ZHEMICYL+Y $2111.2458549 .451-19.764$

END GEOM

READ BNDS $X F C=S P E C$ YFC $=S P E C$ END BNDS

READ PLOT TTL=!UO2_10_8_D_D_INF_OI

$P L T=Y E S$ PIC $=M I X T U R E X \bar{U} L=0$ YUL $=35$ ZUL $=0$

$X L R=110 \quad Y L R=-35 \quad Z L R=0$

$\cup A X=1$ VDN $=-1 \quad N A X=130 \quad L P I=8$

$\mathrm{NCH}=$ ! UF.W ! END PLOT

END DATA

END

$=$ CSAS25

UO2 C 8 D D INF 2

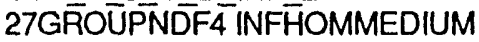

U-234 $10.02 .5829 \mathrm{E}-04$

U-235 $10.01 .6734 \mathrm{E}-02$

U-238 10.01 .4377 E-04

$010.03 .4272 E-02$

CARBONSTEEL 21.0 END

$\mathrm{AL} 30.05 .54-4 \quad$ END

CA $30.05 .30-3 \quad$ END

C $30.06 .59-3 \quad$ END

H 30.0 .0133 END

O 30.0 .0457 END

FE $30.03 .59-4$ END

MG 30.03.25-3 END

SI $30.08 .43-3$ END

NA $30.03 .89-4 \quad$ END

$S \quad 30.01 .09-4$ END

$\mathrm{H} 2 \mathrm{O} 41.0$ END

END COMP

UO2 C 8 D D INF 2

READ $\overline{P A} \bar{A} A \bar{M} \overline{R U N}=\bar{Y}$ ES END PARAM

READ GEOM

UNIT 1

ZHEMICYL+X $117.62-1.2335 E+01-19.05$

ZHEMICYL+X $017.622 P 19.05$

ZHEMICYL+X $217.6992 P 19.129$

ZHEMICYL+X $01 \quad 10.9537548 .181-19.129$

ZHEMICYL+X $21 \quad 11.2458549 .451-19.764$

CUBOID $31105.5675030 .48-30.4849 .451$

$-35.004$

HOLE 2105.567500

HOLE 352.7837530 .480

HOLE $452.78375-30.480$

UNIT 2

ZHEMICYL-X $117.62-1.2335 E+01-19.05$

ZHEMICYL-X $017.622 P 19.05$

ZHEMICYL-X $217.6992 P 19.129$

ZHEMICYL-X $0110.9537548 .181-19.129$

ZHEMICYL-X 2111.2458549 .451 -19.764

UNIT 3

ZHEMICYL-Y 117.62 -1.2335E+01-19.05

ZHEMICYL-Y 017.62 2P19.05

ZHEMICYL-Y $217.6992 P 19.129$

ZHEMICYL-Y 0110.9537548 .181 -19.129

ZHEMICYL-Y $21 \quad 11.2458549 .451-19.764$ 
UNIT 4

ZHEMICYL+Y 117.62 -1.2335E+01 -19.05

ZHEMICYL+Y 017.62 2P19.05

ZHEMICYL+Y $217.6992 P 19.129$

ZHEMICYL+Y $0110.9537548 .181-19.129$

ZHEMICYL+Y $21 \quad 11.2458549 .451-19.764$

END GEOM

READ BNDS XFC $=S P E C$ YFC $=S P E C$ END BNDS

READ PLOT TTL=IUO2 $108 \mathrm{D} D$ INF 2!

$P L T=Y E S P I C=M I X T U R \bar{E} X \bar{U} L=0 \bar{Y} \bar{L}=3 \overline{5} \quad \mathrm{ZUL}=0$

$X L R=110 Y L R=-35 Z L R=0$

$U A X=1$ VDN $=-1 \quad N A X=130 \quad L P I=8$

$\mathrm{NCH}=$ ! UF.W $!$ END PLOT

END DATA

END

\section{$=$ CSAS 25}

UO2 C_8 D_D_INF 4

27GROOUPNDF4 INFHOMMEDIUM

U-234 $10.01 .9902 E-04$

U-235 $10.01 .2894 E-02$

U-238 $10.01 .1078 E-04$

O $10.02 .6407 E-02$

CARBONSTEEL 21.0

AL $30.05 .54-4$

CA $30.05 .30-3$

C $30.06 .59-3$

H 30.0 .0133

O 30.0 .0457

FE $30.03 .59-4$

MG $30.03 .25-3$

SI $30.08 .43-3$

NA $30.03 .89-4$

S $30.01 .09-4$

$\mathrm{H} 2 \mathrm{O} 41.0$

END COMP

UO2 C 8 D D INF 4

REA $\bar{D} \overline{P A} \bar{R} A \bar{M} \bar{R} U N=\bar{Y}=S$ END PARAM

READ GEOM

UNIT 1

ZHEMICYL+X $117.62-1.0335 E+01-19.05$

ZHEMICYL+X 017.62 2P 19.05

ZHEMICYL+X $217.6992 P 19.129$

ZHEMICYL+X $0110.9537548 .181-19.129$

ZHEMICYL+X $21 \quad 11.2458549 .451-19.764$

CUBOID $31105.5675030 .48-30.4849 .451$

$-35.004$

HOLE 2105.567500

HOLE 352.7837530 .480

HOLE $452.78375-30.480$

UNIT 2

ZHEMICYL-X $117.62-1.0335 E+01-19.05$

ZHEMICYL-X 017.62 2P19.05

ZHEMICYL-X 217.699 2P19.129

ZHEMICYL-X $0110.9537548 .181-19.129$

ZHEMICYL-X 2111.2458549 .451 -19.764

UNIT 3

ZHEMICYL-Y 117.62 -1.0335E+01-19.05

ZHEMICYL-Y 017.62 2P19.05

ZHEMICYL-Y 217.699 2.P19.129

ZHEMICYL-Y $0110.9537548 .181-19.129$

ZHEMICYL-Y $2111.2458549 .451-19.764$

UNT 4

ZHEMICYL+Y $117.62-1.0335 E+01-19.05$
ZHEMICYL+YO $17.622 P 19.05$

ZHEMICYL+Y $217.6992 P 19.129$

ZHEMICYL+Y O $110.9537548 .181-19.129$

ZHEMICYL+Y $2111.2458549 .451-19.764$

END GEOM

READ BNDS XFC $=S P E C$ YFC $=S P E C$ END BNDS

READ PLOT TTL=IUO2 108 D D INF $4 !$

$P L T=Y E S$ PIC $=M \mid X T U R \bar{E} X \bar{U} L=0 \overline{Y U L}=35 \mathrm{ZUL}=0$

$X L R=110$ YLR $=-35 \quad Z L R=0$

$U A X=1$ VDN $=-1 \quad N A X=130 \quad L P I=8$

$N C H=! U F . W \mid E N D$ PLOT

END DATA

END

\section{$=$ COAS25}

UO2_C_8_D_D_INF 8

27GROUUPNDF4 INFHOMMEDIUM

U-234 $10.01 .3641 \mathrm{E}-04$

U-235 $10.08 .8375 E-03$

U-238 $10.07 .5929 E-05$

$010.01 .8100 E-02$

CARBONSTEEL 21.0

AL $30.05 .54-4$

CA $30.05 .30-3$

C $30.06 .59-3$

H 30.0 .0133

O 30.0 .0457

FE $30.03 .59-4$

MG $30.03 .25-3$

SI $30.08 .43-3$

NA $30.03 .89-4$

S $30.01 .09-4$

$\mathrm{H} 2 \mathrm{O} 41.0$

END COMP

UO2 C 8 D D INF 8

READ PA $\bar{P} \bar{R} \bar{M} \bar{R} U N=\bar{Y}=S$ END PARAM

READ GEOM

UNIT 1

ZHEMICYL+X $117.62-6.3350 E_{+} 00-19.05$

ZHEMICYL+X $017.622 \mathrm{P} 19.05$

ZHEMICYL+X 217.699 2P19.129

ZHEMICYL+X $0110.9537548 .181-19.129$

ZHEMICYL+X $21 \quad 11.2458549 .451-19.764$

CUBOID $31105.5675030 .48-30.4849 .451$ $-35.004$

HOLE 2105.567500

HOLE 352.7837530 .480

HOLE $452.78375-30.480$

UNIT 2

ZHEMICYL-X $117.62-6.3350 E+00-19.05$

ZHEMICYL-X 017.62 2P19.05

ZHEMICYL-X $217.6992 P 19.129$

ZHEMICYL-X $0110.9537548 .181-19.129$

ZHEMICYL-X $2111.2458549 .451-19.764$

UNIT 3

ZHEMICYL-Y $117.62-6.3350 E+00-19.05$

ZHEMICYL-Y 017.62 2P19.05

ZHEMICYL-Y 217.699 2P19.129

ZHEMICYL-Y $0110.9537548 .181-19.129$

ZHEMICYL-Y $2111.2458549 .451-19.764$

UNIT 4

ZHEMICYL+Y $117.62-6.3350 E+00-19.05$

ZHEMICYL+Y $017.622 \mathrm{P} 19.05$

ZHEMICYL+Y $217.6992 P 19.129$ 
ZHEMICYL+YO $110.9537548 .181 \cdot 19.129$ ZHEMICYL+Y $2111.2458549 .451-19.764$ END GEOM

READ BNDS XFC=SPEC YFC=SPEC END BNDS READ PLOT TTL=IUO2_10_8_D_D_INF_8! $P L T=Y E S$ PIC $=M I X T U R \bar{E} X \bar{U} L=0 \bar{Y} \bar{L}=3 \overline{5} \mathrm{ZUL}=0$ $X L R=110$ YLR $=-35$ ZLR $=0$

$U A X=1$ VDN $=-1 \quad N A X=130 L P \mid=8$

$\mathrm{NCH}=\mid$ UF.W $\mid$ END PLOT

END DATA

END

$=$ CSAS25

UO2 C 8 D D INF 10

27GR̄OUPNDF4 INFH̄OMMEDIUM

U-234 $10.01 .1787 E-04$

U-235 $10.07 .6363 E-03$

U-238 $10.06 .5609 E-05$

END

O $10.01 .5640 \mathrm{E}-02$

CARBONSTEEL 21.0

AL $30.05 .54-4$

CA $30.05 .30-3$

C $30.06 .59-3$

H 30.0 .0133

O 30.0 .0457

FE $30.03 .59-4$

MG $30.03 .25-3$

SI $30.08 .43-3$

NA $30.03 .89-4$

S $30.01 .09-4$

END

END

END

END

END

END

END

END

END

END

END

END

END

$\mathrm{H} 2 \mathrm{O} 41.0$

END COMP

UO2_C 8 D D INF 10

READ PARAM $\bar{R} \bar{R} \mathrm{~N}=\bar{Y}=S$ END PARAM

READ GEOM

UNIT 1

ZHEMICYL+X $117.62-4.3350 E+00-19.05$

ZHEMICYL+X $017.622 P 19.05$

ZHEMICYL+X $217.6992 P 19.129$

ZHEMICYL+X $0110.9537548 .181-19.129$

ZHEMICYL+X $2411.2458549 .451-19.764$

CUBOID $31105.5675030 .48-30.4849 .451$

$-35.004$

HOLE 2105.567500

HOLE 352.7837530 .480

HOLE $452.78375-30.480$

UNIT 2

ZHEMICYL-X $117.62-4.3350 E+00-19.05$

ZHEMICYL-X 017.62 2P19.05

ZHEMICYL-X 217.699 2P19.129

ZHEMICYL-X $0110.9537548 .181-19.129$

ZHEMICYL-X 2111.2458549 .451 -19.764 UNTT 3

ZHEMICYL-Y $117.62-4.3350 E+00-19.05$

ZHEMICYL-Y 017.62 2P19.05

ZHEMICYL-Y 217.699 2P19.129

ZHEMICYL-Y $0110.9537548 .181-19.129$

ZHEMICYL-Y $21 \quad 11.2458549 .451$-19.764

UNIT 4

$Z H E M I C Y L+Y 117.62-4.3350 E+00-19.05$

ZHEMICYL+Y $017.622 \mathrm{P} 19.05$

ZHEMICYL+Y $217.6992 P 19.129$

ZHEMICYL+Y $0110.9537548 .181-19.129$

ZHEMICYL+Y $21 \quad 11.2458549 .451-19.764$
END GEOM

READ BNDS XFC $=S P E C$ YFC $=S P E C$ END BNDS

READ PLOT TTL=IUO2_10_8_D_D_INF_101

PLT $=$ YES PIC $=M I X T U R \bar{E} X \bar{U} L=0 \bar{Y} U \bar{L}=35 \mathrm{ZUL}=0$

$X L R=110 \quad Y L R=-35 \quad Z L R=0$

$U A X=1$ VDN $=-1 \quad N A X=130 L P \mid=8$

$\mathrm{NCH}=$ ! UF.W ! END PLOT

END DATA

END

$=$ CSAS25

UO2 C 8 D D INF 20

27GR̄OÜPNDFA INFḦOMMEDIUM

U-234 10.07 .0177 E-05

U-235 $10.04 .5466 \mathrm{E}-03$

U-238 $10.03 .9063 E-05$

O $10.09 .3116 \mathrm{E}-03$

CARBONSTEEL 21.0

AL $30.05 .54-4$

CA $30.05 .30-3$

C $30.06 .59-3$

H 30.0 .0133

O 30.0 .0457

FE $30.03 .59-4$

MG $30.03 .25-3$

SI $30.08 .43-3$

NA $30.03 .89-4$

S $30.01 .09-4$

$\mathrm{H} 2 \mathrm{O} 41.0$

END COMP

UO2 C 8D D INF 20

REA $\bar{D} \overline{P A} \bar{R} A \bar{M} \bar{R} U N=\bar{Y}=S$ END PARAM

READ GEOM

UNIT 1

ZHEMICYL+X $117.625 .6650 E+00-19.05$

ZHEMICYL+X 017.62 2P19.05

ZHEMICYL+X 217.699 2P19.129

ZHEMICYL+X $0110.9537548 .181-19.129$

ZHEMICYL+X $21 \quad 11.2458549 .451-19.764$

CUBOID $31105.5675030 .48-30.4849 .451$

$-35.004$

HOLE 2105.567500

HOLE 352.7837530 .480

HOLE $452.78375-30.480$

UNIT 2

ZHEMICYL-X $117.625 .6650 E+00-19.05$

ZHEMICYL-X 017.62 2P19.05

ZHEMICYL-X 217.699 2P19.129

ZHEMICYL-X $0110.9537548 .181-19.129$

ZHEMICYL-X $2111.2458549 .451-19.764$

UNIT 3

ZHEMICYL-Y 117.62 5.6650E+00 -19.05

ZHEMICYL-Y 017.62 2P19.05

ZHEMICYL-Y 217.699 2P19.129

ZHEMICYL-Y $0110.9537548 .181-19.129$

ZHEMICYL-Y $21 \quad 11.2458549 .451-19.764$

UNIT 4

ZHEMICYL+Y $117.625 .6650 E+00-19.05$

ZHEMICYL+Y 017.62 2P19.05

ZHEMICYL+Y $217.6992 P 19.129$

ZHEMICYL+Y $0110.9537548 .181-19.129$

ZHEMICYL+Y $21 \quad 11.2458549 .451-19.764$

END GEOM

READ BNDS XFC=SPEC YFC $=S P E C$ END BNDS 
READ PLOT TTL=|UO2 108 D D INF 201 $P L T=Y E S$ PIC $=M I X T U R \bar{E} X \bar{U} L=0 \bar{Y} U \bar{L}=35 \mathrm{ZUL}=0$ $X L R=110 Y L R=-35 Z Z L=0$

$U A X=1$ VDN $=-1 \quad N A X=130 \quad L P I=8$

$\mathrm{NCH}=$ ! UF.W I END PLOT

END DATA

END

\section{=CSAS25}

UO2 C 8 D D INF 33

27GR̄OUPNDF4 INFHOMMEDIUM

U-234 10.0 4.5523E-05

U-235 $10.02 .9493 E-03$

$\mathrm{U}-23810.02 .5340 \mathrm{E}-05$

O $10.06 .0403 E-03$

CARBONSTEEL 21.0

AL $\quad 30.05 .54-4$

CA $30.05 .30-3$

C $30.06 .59-3$

H 30.0 .0133

O 30.0 .0457

FE $30.03 .59-4$

MG $30.03 .25-3$

SI $30.08 .43-3$

NA $30.03 .89-4$

S $30.01 .09-4$

$\mathrm{H} 2 \mathrm{O} 41.0$

END COMP

UO2 C. 8 D D INF 33

REA $\bar{D} \overline{P A} \bar{R} A \bar{M} \bar{R} U N=\bar{Y}=S$ END PARAM

READ GEOM

UNIT 1

ZHEMICYL+X $117.622 P 19.05$

ZHEMICYL+X $217.6992 P 19.129$

ZHEMICYL+X $0110.9537548 .181-19.129$

ZHEMICYL+X $21 \quad 11.2458549 .451-19.764$

CUBOID $31105.5675030 .48-30.4849 .451$

$-35.004$

HOLE 2105.567500

HOLE 352.7837530 .480

HOLE $452.78375-30.480$

UNTT 2

ZHEMICYL-X $117.622 \mathrm{P} 19.05$

ZHEMICYL-X $217.6992 P 19.129$

ZHEMICYL-X $0110.9537548 .181-19.129$

ZHEMICYL-X 2111.2458549 .451 -19.764

UNT 3

ZHEMICYL-Y 117.62 2P19.05

ZHEMICYL-Y 217.699 2P19.129

ZHEMICYL-Y $0110.9537548 .181-19.129$

ZHEMICYL-Y $21 \quad 11.2458549 .451$-19.764

UNIT 4

ZHEMICYL+Y $117.622 P 19.05$

ZHEMICYL+Y $217.6992 P 19.129$

ZHEMICYL+Y O $110.9537548 .181-19.129$

ZHEMICYL+Y $21 \quad 11.2458549 .451-19.764$

END GEOM

READ BNDS $X F C=S P E C$ YFC $=S P E C$ END BNDS

READ PLOT TTL=IUO2_10_8_D_D_INF_33!

PLT=YES PIC $=M I X T U R \bar{E} X \bar{U} L=0 \bar{Y} \bar{U} \bar{L}=3 \overline{5} Z \mathrm{ZUL}=0$

$X L R=110 Y L R=-35 Z Z R=0$

$U A X=1 \quad V D N=-1 \quad N A X=130 \quad L P I=8$

$N C H=|U F . W| E N D$ PLOT

END DATA
END

\section{OXIDES IN 5-INCH CANISTERS}

-CSAS25

U3O8_15_3.47_W D

27GRŌUPNDF $\overline{4}$ INFHOMMEDIUM

U-234 $10.05 .4837 E-05$

U-235 $10.03 .5527 E-03$

U-238 $10.03 .0524 E-05$

H $10.05 .4571 E-02$

O $10.03 .6987 E-02$

CARBONSTEEL 21.0

AL $30.05 .54-4$

CA $30.05 .30-3$

C $30.06 .59-3$

H3 0.0 .0133

O 30.0 .0457

FE $30.03 .59-4$

MG $30.03 .25-3$

SI $30.08 .43-3$

NA $30.03 .89-4$

S $30.01 .09-4$

$\mathrm{H} 2 \mathrm{O} 41.0$

END COMP

U3O8 $15 \quad 3.47$ W D

READPA $\bar{R} A M \bar{R} U \bar{N}=$ YES END PARAM

READ GEOM

CYLINDER $116.350 .718221-19.05$

CYLINDER $016.35019 .05-19.05$

CYLINDER $216.42919 .129-19.129$

CYLINDER $4110.9537548 .181-19.129$

CYLINDER $2111.2458549 .451-19.764$

CUBOID $3350-5050-5049.451-50.549$

END GEOM

READ BNDS XFC=VAC YFC=VAC END BNDS

READ PLOT TTL $=\mid U 308$ 15_3.47_W_D!

PLT $=$ YES PIC $=$ MIXTURE XUL $=-50^{-}$YUL $=00$

$Z U L=50$ XLR $=50$ YLR $=00$ ZLR $=-30$

$U A X=1$ WDN $=-1 \quad N A X=130 \quad \mathrm{~L} . \mathrm{PI}=8$

$\mathrm{NCH}=$ ! UFCW IEND PLOT

END DATA

END

$=$ CSAS25

UO2 103.47 W D

27GR̄OUPNDF̄4 INFHOMMEDIUM

U-234 $10.08 .1100-5$

U-235 $10.05 .254-3$

END

U-238 1 0.0 4.51-5

H $10.05 .3804-2$

O $10.03 .7663-2$

CARBONSTEEL $21.0 \quad$ END

AL $30.05 .54-4$ END

CA $30.05 .30-3 \quad$ END

C $30.06 .59-3$

H 30.0 .0133 END

030.0 .0457 END

FE $30.03 .59-4$ END

MG $30.03 .25-3$ END

SI $30.08 .43-3 \quad$ END

NA $30.03 .89-4$ END

S $30.01 .09 .4 \quad$ END

$\mathrm{H} 2 \mathrm{O} 41.0$ END 
END COMP

UO2 10 3.47 W D

READ PARAM $\bar{R} \overline{\mathrm{N}}=$ =YES END PARAM

READ GEOM

CYLINDER 116.350 -5.68347 -19.05

CYLINDER $016.35019 .05-19.05$

CYLINDER $216.42919 .129-19.129$

CYLINDER $4110.9537548 .181-19.129$

CYLINDER $2111.2458549 .451-19.764$

CUBOID $3350 \quad-5050 \quad-50 \quad 49.451 \quad-50.549$

END GEOM

READ BNDS XFC $=V A C$ YFC $=V A C$ END BNDS

READ PLOT TTL=IUO2 $103.47 \mathrm{~W}$ DI

PLT $=$ YES PIC $=$ MIXTURE $X \bar{U} L=-50$ YUL $=00$

$Z U L=50 X L R=50$ YLR $=00 Z Z R=-30$

$U A X=1$ WDN $=-1$ NAX $=130 \quad L P I=8$

$\wedge, \mathrm{SH}=1$ UFCW ! END PLOT

END DATA

END

$=$ CSAS 25

UO2 105.56 D D

27GROUPNDF4 INFHOMMEDIUM

U-234 $10.08 .1100-5$

U-235 $10.05 .254-3$

END

END

U-238 $10.04 .51-5$

H 10.05 .3804 .2

O $10.03 .7663-2$

CARBONSTEEL 21.0

AL $30.05 .54-4$

CA $30.05 .30-3$

C $30.06 .59-3$

H 30.0 .0133

030.0 .0457

FE $30.03 .59-4$

MG $30.03 .25-3$

SI $30.08 .43-3$

NA $30.03 .89-4$

$S 30.01 .09-4$

$\mathrm{H} 2 \mathrm{O} 41.0$

END COMP

UO2 $105.56 \mathrm{D} D$

REA $\bar{D}$ PARAM $\bar{A} \bar{U} \bar{N}=$ YES END PARAM READ GEOM

CYLINDER $116.3502 .3364508-19.05$

CYLINDER $016.35019 .05-19.05$

CYLINDER 216.429 19.129-19.129

CYLINDER $0110.9537548 .181-19.129$

CYLINDER $2111.2458549 .451-19.764$

CUBOID $3350-5050-5049.451$-50.549

END GEOM

READ BNDS XFC $=V A C$ YFC $=V A C$ END BNDS

READ PLOT TTL=!UO2 10 5.56 D D!

$P L T=Y E S P I C=M I X T U R \bar{E} \times \overline{U L}=-50 \overline{Y U L}=00$

$Z U L=50 X L R=50$ YLR $=00 \quad Z L R=-30$

$U A X=1 W D N=-1 \quad N A X=130 \quad L P I=8$

$\mathrm{NCH}=$ ! UFCW $!$ END PLOT

END DATA

END

\section{=CSAS25}

UO2 105.56 D D INF

27GROUPNDF4 INFHOMMEDIUM

U-234 $10.08 .1100-5$
U-235 10.05 .254 .3

END

U.238 $10.04 .51-5$

END

H $10.05 .3804-2$

$010.03 .7663-2$

CARBONSTEEL 21.0

END

AL $30.05 .54-4$

CA $30.05 .30-3$

C $30.06 .59-3$

H3 0.0 .0133

O 30.0 .0457

FE $30.03 .59-4$

MG $30.03 .25-3$

SI $30.08 .43-3$

NA $30.03 .89-4$

S $30.01 .09-4$

$\mathrm{H} 2 \mathrm{O} 41.0$

END COMP

UO2_10_5.56_D_D_INF

READ GEOM

UNIT 1

ZHEMICYL+X 116.3502 .3364508 -19.05

ZHEMICYL+X $016.3502 P 19.05$

ZHEMICYL+X $216.4292 \mathrm{P} 19.129$

ZHEMICYL+X $0110.9537548 .181-19.129$

ZHEMICYL+X $2111.2458549 .451-19.764$

CUBOID $31105.5675030 .48-30.4849 .451$

$-35.004$

HOLE 2105.567500

HOLE 352.7837530 .480

HOLE $452.78375-30.480$

UNIT 2

ZHEMICYL-X $116.3502 .3364508-19.05$

ZHEMICYL-X 016.350 2P19.05

ZHEMICYL-X 216.429 2P19.129

ZHEMICYL-X 0110.9537548 .181 -19.129

ZHEMICYL-X $2111.2458549 .451-19.764$

UNIT 3

ZHEMICYL-Y 116.3502 .3364508 -19.05

ZHEMICYL-Y 016.350 2P19.05

ZHEMICYL-Y 216.429 2P19.129

ZHEMICYL-Y $0110.9537548 .181-19.129$

ZHEMICYL-Y $2111.2458549 .451-19.764$

UNIT 4

$Z H E M I C Y L+Y 116.3502 .3364508-19.05$

ZHEMICYL+YO 16.3502 P 19.05

ZHEMICYL+Y $216.4292 P 19.129$

ZHEMICYL+Y $0110.9537548 .181-19.129$

ZHEMICYL+Y2111.24585 49.451-19.764

END GEOM

READ BNDS XFC=SPEC YFC=SPEC END BNDS

READ PLOT TTL $=$ !UO2 $10 \quad 5.56$ D D INF!

$P L T=Y E S P I C=M I X T U R \bar{E} X \bar{U} L=0 \bar{Y} U \bar{L}=\overline{3} 5 \mathrm{ZUL}=0$

$X L R=110$ YLR $=-35$ ZLR $=0$

$U A X=1 \quad V D N=-1 \quad N A X=130 L P I=8$

$N C H=$ ! UF. W IEND PLOT

END DATA

END

$=$ CSAS25

UO2 $105.56 \mathrm{~W} D$

27GR̄OUPNDF4 INTFHOMMEDIUM

U-234 $10.08 .1100-5$

U-235 $10.05 .254-3$

END

U-238 $10.04 .51-5$
END

END 


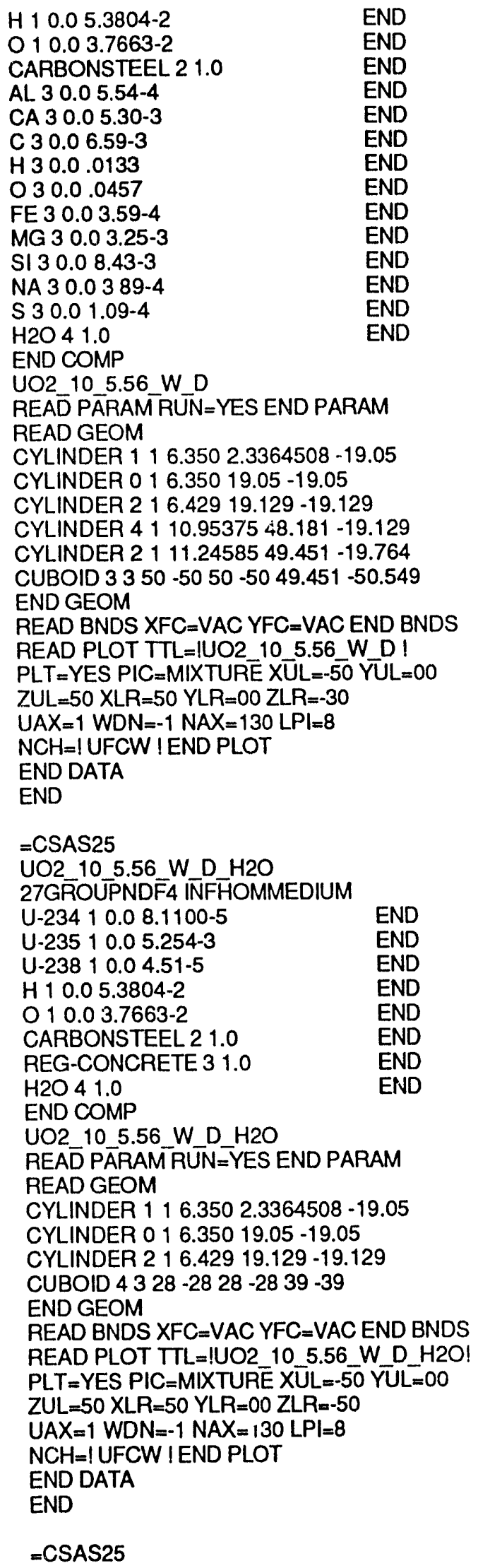

UO2_10_5.56_W_D_INF

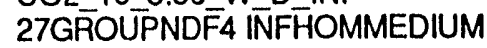
U-234 $10.08 .1100-5$

U-235 $10.05 .254-3$

U-238 $10.04 .51-5$

H $10.05 .3804-2$

O $10.03 .7663-2$

CARBONSTEEL 21.0

AL $30.05 .54-4$

CA $30.05 .30-3$

C $30.06 .59-3$

H 30.0 .0133

030.0 .0457

FE $30.03 .59-4$

MG $30.03 .25-3$

SI $30.08 .43-3$

NA $30.03 .89-4$

S $30.01 .09-4$

$\mathrm{H} 2 \mathrm{O} 41.0$

END COMP

UO2 10_5.56_W_D_INF

READ GEOM

UNIT 1

ZHEMICYL+X $116.3502 .3364508-19.05$

ZHEMICYL+X $016.3502 P 19.05$

ZHEMICYL+X $216.4292 P 19.129$

ZHEMICYL+X $4110.9537548 .181-19.129$

ZHEMICYL+X $2111.2458549 .451-19.764$

CUBOID $31105.5675030 .48-30.4849 .451$

$-35.004$

HOLE 2105.567500

HOLE 352.7837530 .480

HOLE $452.78375-30.480$

UNIT 2

ZHEMICYL-X 116.3502 .3364508 -19.05

ZHEMICYL-X 016.350 2P19.05

ZHEMICYL-X 216.429 2P19.129

ZHEMICYL-X $4110.9537548 .181-19.129$

ZHEMICYL-X 2111.2458549 .451 -19.764

UNIT 3

ZHEMICYL-Y 116.3502 .3364508 -19.05

ZHEMICYL-Y 016.350 2P19.05

ZHEMICYL-Y 216.429 2P19.129

ZHEMICYL-Y $4110.9537548 .181-19.129$

ZHEMICYL.Y $2111.2458549 .451-19.764$

UNIT 4

$Z H E M I C Y L+Y 116.3502 .3364508-19.05$

ZHEMICYL+Y $016.3502 P 19.05$

ZHEMICYL+Y $216.4292 P 19.129$

ZHEMICYL+Y $4110.9537548 .181-19.129$

ZHEMICYL+Y $2111.2458549 .451-19.764$

END GEOM

READ BNDS XFC $=S P E C$ YFC $=S P E C$ END BNDS

READ PLOT TTL=!UO2_10_5.56_W_D_INF!

PLT $=$ YES PIC $=$ MIXTURE $X \bar{U} L=0 \bar{Y} U \bar{L}=\overline{35} Z U L=0$

$X L R=110 Y L R=-35 \quad Z L R=0$

$U A X=1$ VDN $=-1 \quad N A X=130 \quad L P I=8$

$\mathrm{NCH}=$ ! UF.W ! END PLOT

END DATA

END

$=$ CSAS25

UO2 10 5.56 W W W

27GROOUPNDF4 INTFHOMMEDIUM 


\begin{tabular}{|c|c|}
\hline 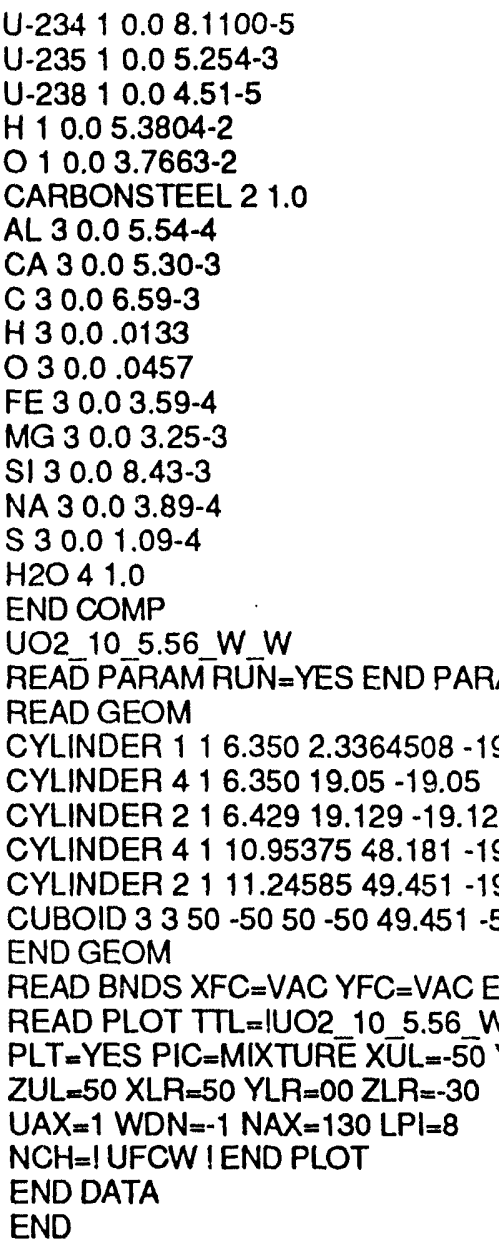 & $\begin{array}{l}\text { END } \\
\text { END } \\
\text { END } \\
\text { END } \\
\text { END } \\
\text { END } \\
\text { END } \\
\text { END } \\
\text { END } \\
\text { END } \\
\text { END } \\
\text { END } \\
\text { END } \\
\text { END } \\
\text { END } \\
\text { END } \\
\text { END }\end{array}$ \\
\hline 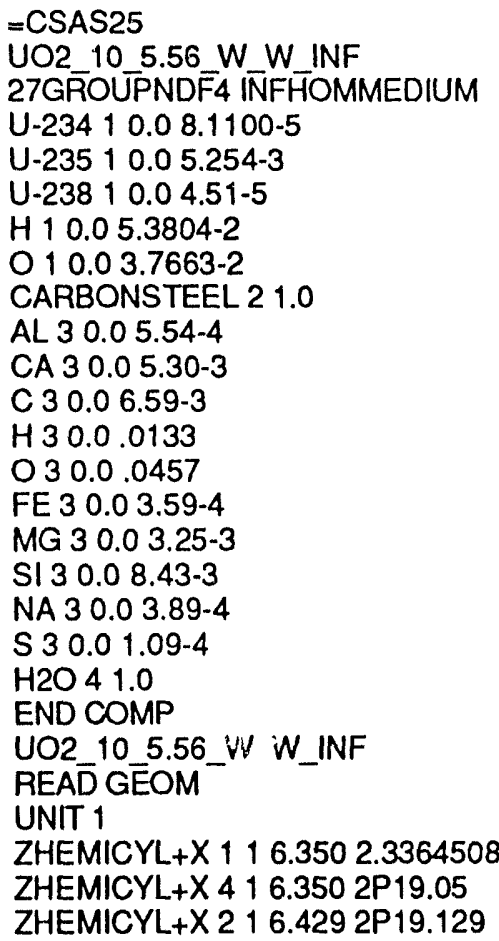 & $\begin{array}{l}\text { END } \\
\text { END } \\
\text { END } \\
\text { END } \\
\text { END } \\
\text { END } \\
\text { END } \\
\text { END } \\
\text { END } \\
\text { END } \\
\text { END } \\
\text { END } \\
\text { END } \\
\text { END } \\
\text { END } \\
\text { END } \\
\text { END }\end{array}$ \\
\hline
\end{tabular}

ZHEMICYL+X $4110.9537548 .181-19.129$ ZHEMICYL+X $2111.2458549 .451-19.764$ CUBOID $31105.5675030 .48-30.4849 .451$ $-35.004$

HOLE 2105.567500

HOLE 352.7837530 .480

HOLE $452.78375-30.480$

UNIT 2

ZHEMICYL-X $116.3502 .3364508-19.05$

ZHEMICYL-X 416.350 2P19.05

ZHEMICYL-X $216.4292 P 19.129$

ZHEMICYL-X 4110.9537548 .181 -19.129

ZHEMICYL-X $2111.2458549 .451-19.764$ UNTT 3

ZHEMICYL-Y $116.3502 .3364508-19.05$

ZHEMICYL-Y $416.3502 P 19.05$

ZHEMICYL-Y 216.429 2P19.129

ZHEMICYL-Y 4110.9537548 .181 -19.129

ZHEMICYL-Y $2111.2458549 .451-19.764$

UNIT 4

ZHEMICYL+Y $116.3502 .3364508-19.05$

$Z H E M I C Y L+Y 416.3502 P 19.05$

$Z H E M I C Y L+Y 216.4292 P 19.129$

ZHEMICYL+Y $4110.9537548 .181-19.129$

ZHEMICYL+Y $2111.2458549 .451-19.764$

END GEOM

READ BNDS XFC=SPEC YFC=SPEC END BNDS

READ PLOT TTL $=! U O 2$ 10_5.56_W_W_INF!

PLT $=Y E S$ PIC $=$ MIXTURE $X \bar{L} L=0$ YUL $=35$ ZUL $=0$

$X L R=110$ YLR $=-35$ ZLR $=0$

$U A X=1$ VDN $=-1 \quad N A X=130 \quad L P I=8$

$\mathrm{NCH}=$ ! UF.W I END PLOT

END DATA

END

$=$ CSAS 25

UO2 107.29 W D

27GR̄OUPNDF4 INFHOMMEDIUM

U-234 $10.08 .1100-5$

U-235 $10.05 .254-3$

U-238 $10.04 .51-5$

H $10.05 .3804-2$

$010.03 .7663-2$

CARBONSTEEL $21.0 \quad$ END

AL $30.05 .54-4 \quad$ END

CA $30.05 .30-3 \quad$ END

C $30.06 .59-3 \quad$ END

H3 0.0 .0133 END

030.0 .0457 END

FE $30.03 .59-4 \quad$ END

MG $30.03 .25-3$ END

SI $30.08 .43-3 \quad$ END

NA $30.03 .89-4 \quad$ END

$S 30.01 .09-4 \quad$ END

$\mathrm{H} 2 \mathrm{O} 41.0$ END

END COMP

UO2 107.29 W D

READ PARAMRUN $=$ YES END PARAM

READ GEOM

CYLINDER $116.3509 .01972-19.05$

CYLINDER $016.35019 .05-19.05$

CYLINDER 216.429 19.129-19.129

CYLINDER $4110.9537548 .181-19.129$

CYLINDER $2111.2458549 .451-19.764$ 
CUBOID $3350-5050-5049.451-50.549$ END GEOM

READ BNDS XFC $=V A C$ YFC $=V A C$ END BNDS

READ PLOT TTL $=! U O 2$ 10 7.29_W_DI

PLT $=$ YES PIC $=$ MIXTURE XUL $=-50$ YUL $=00$

$Z U L=50$ XLR $=50$ YLR $=00$ ZLR $=-30$

$U A X=1$ WDN $=-1$ NAX $=130 \mathrm{LPI}=8$

$\mathrm{NCH}=\mid$ UFCW IEND PLOT

END DATA

END

\section{$=$ CSAS25}

UO2_10_8_W_D

27GR̄OUPNDDF4 INFHOMMEDIUM

U-234 10.08 .1099E-05

U-235 $10.05 .2542 E-03$

END

U-238 $10.04 .5142 E-05$

END

H 10.0 5.3804E-02

O $10.03 .7663 E-02$

CARBONSTEEL 21.0

AL 3 0.0 5.54-4

CA $30.05 .30-3$

C $30.06 .59-3$

H 30.0 .0133

030.0 .0457

FE $30.03 .59-4$

MG $30.03 .25-3$

SI $30.08 .43-3$

NA $30.03 .89-4$

S $30.01 .09-4$

$\mathrm{H} 2 \mathrm{O} 41.0$

END COMP

UO2 10 8 W D

READ PA $\bar{A} \overline{R A} \bar{M} \mathrm{RUN}=$ YES END PARAM

READ GEOM

CYLINDER $116.35011 .7467-19.05$

CYLINDER $416.35019 .05-19.05$

CYLINDER $216.42919 .129-19.129$

CYLINDER $4110.9537548 .181-19.129$

CYLINDER 21 11.24ं85 $49.451-19.764$

CUBOID $3350-5050-50 \quad 49.451-50.549$

END GEOM

READ BNDS XFC $=V A C$ YFC $=V A C$ END BNDS

READ PLOT TTL=!UO2 $108 \mathrm{~W} \mathrm{D}$ !

PLT $=$ YES PIC $=$ MIXTURE XULL $=-5 \overline{0}$ YUL $=00$

$Z U L=50 \quad X L R=50 \quad Y L R=00 Z L R=-30$

$U A X=1$ WDN $=-1$ NAX $=130 L P I=8$

$\mathrm{NCH}=$ ! UFCW ! END PLOT

END DATA

END

-CSAS25

UO2 108 W M 5

27GR̄OUPNDDF4 INFHOMMEDIUM

U-234 $10.06 .9771 E-05$

U-235 $10.04 .5203 E-03$

END

U-238 $10.03 .8837 E-05$

H $10.05 .5628 E-02$

O $10.03 .7072 E-02$

CARBONSTEEL 21.0

AL 3 0.0 5.54-4

CA $30.05 .30-3$

C $30.06 .59-3$

H 30.0 .0133
030.0 .0457

END

FE $30.03 .59-4$

MG $30.03 .25-3$

SI $30.08 .43-3$

NA $30.03 .89-4$

S $30.01 .09-4$

$\mathrm{H} 2 \mathrm{O} 41.0$

END COMP

UO2 108 W M 5

READ PARAM $\bar{R} \overline{U N}=$ YES END PARAM

READ GEOM

CYLINDER $116.35016 .7467-19.05$

CYLINDER $416.35019 .05-19.05$

CYLINDER $216.42919 .129-19.129$

CYLINDER $4110.9537548 .181-19.129$

CYLINDER $2111.2458549 .451-19.764$

CUBOID $3350-5050-5049.451$-50.549

END GEOM

READ BNDS XFC $=V A C$ YFC $=V A C$ END BNDS

READ PLOT TTL $=! U O 2$ 2 10_8_W_M_5!

PLT $=$ YES PIC $=M I X T U R \bar{E} \times \bar{U} L=-50$ YUL $=00$

$Z U L=50 \quad X L R=50$ YLR $=00 \quad Z L R=-30$

$U A X=1$ WDN $=-1 \quad N A X=130 \quad L P \mid=8$

$\mathrm{NCH}=$ !UFCW $\mid$ END PLOT

END DATA

END

=CSAS 25

UO2_10_8 W_M 7

27GR̄OUPNDDF4 INFHOMMEDIUM

U-234 $10.06 .6079 E-05$

$U-23510.04 .2811 E-03$

$\mathrm{U}-23810.03 .6782 \mathrm{E}-05$

H $10.05 .6222 E-02$

O $10.03 .6879 \mathrm{E}-02$

CARBONSTEEL 21.0

AL $30.05 .54-4$

CA $30.05 .30-3$

C $30.06 .59-3$

H3 0.0 .0133

O 30.0 .0457

FE $30.03 .59-4$

MG $30.03 .25-3$

SI $30.08 .43-3$

NA $30.03 .89-4$

S $30,01.09-4$

$\mathrm{H} 2 \mathrm{O} 41.0$

END COMP

UO2_10_8_W_M 7

READ PARAM $R \bar{U} \bar{N}=Y E S$ END PARAM

READ GEOM

CYLINDER $116.35018 .7467-19.05$

CYLINDER $416.35019 .05-19.05$

CYLINDER $216.42919 .129-19.129$

CYLINDER $4110.9537548 .181-19.129$

CYLINDER $2111.2458549 .451-19.764$

CUBOID $3350-5050-5049.451$-50.549

END GEOM

READ BNDS XFC $=V A C$ YFC $=V A C$ END BNDS

READ PLOT $T T L=! U O 2 \quad 108 \mathrm{~W} M 7 !$

PLT $=$ YES PIC $=$ MIXTURE $X \bar{U} L=-50$ YUL $=00$

$Z U L=50$ XLR $=50$ YLR $=00 \quad Z L R=-30$

$U A X=1$ WDN $=-1 \quad N A X=130 \quad L P \mid=8$

$N C H=$ IUFCW IEND PLOT 
END DATA

END

=CSAS25

UO2 10 FULL D D

27GR̄OUPNDFA INFHOMMEDIUM

U.23410.08 $11100-5$

U-235 $10.05 .254-3$

END

U-238 $10.04 .51-5$

END

END

H $10.05 .3804-2$

$010.03 .7663-2$

END

END

CARBONSTEEL 21.0 END

AL 3 0.0 5.54-4

END

CA $30.05 .30-3$

C $30.06 .59-3$

H 30.0 .0133

END

END

O 30.0 .0457

FE $30.03 .59-4$

END

END

END

END

MG $30.03 .25-3$

END

NA $30.03 .89-4$

END

$S 30.01 .09-4$

END

$\mathrm{H} 2 \mathrm{O} 41.0$

END COMP

UO2 10 FULL D D

READ PARAM $\bar{R} U \bar{N}=Y$ YES END PARAM

READ GEOM

CYLINDER $116.35019 .05-19.05$

CYLINDER $216.42919 .129-19.129$

CYLINDER $0110.9537548 .181-19.129$

CYLINDER $2111.2458549 .451-19.764$

CUBOID $3350-5050-5049.451-50.549$

END GEOM

READ BNDS XFC $=V A C$ YFC $=V A C$ END BNDS

READ PLOT TTL=1UO2 10 FULL D DI

PLT $=Y E S$ PIC $=M \mid X T U R \bar{R} \overline{X U L}=-5 \bar{O}$ YUUL $=00$

$Z U L=50 \quad X L R=50$ YLR $=00 \quad Z L R=-30$

$U A X=1$ WDN=-1 NAX=130 LPI $=8$

$\mathrm{NCH}=$ ! UFCW IEND PLOT

END DATA

END

\section{$=$ CSAS 25}

UO2 10 FULL D D INF

27GR̄OUPNDF 4 INFH'OMMEDIUM

U-234 $10.08 .1100-5$

U-235 $10.05 .254-3$

U-238 $10.04 .51-5$

H $10.05 .3804-2$

$010.03 .7663-2$

CARBONSTEEL 21.0

AL 3 0.0 5.54-4

CA $30.05 .30-3$

C $30.06 .59-3$

H 30.0 .0133

O 30.0 .0457

FE $30.03 .59-4$

MG $30.03 .25-3$

SI $30.08 .43-3$

NA $30.03 .89-4$

S $30.01 .09-4$

$\mathrm{H} 2 \mathrm{O} 41.0$

END

END

END

END

END

END

END

END

END

END

END

END

END

END

END

END

END
READ GEOM

UNIT 1

ZHEMICYL+X $116.3502 \mathrm{P} 19.05$

ZHEMICYL+X $216.4292 P 19.129$

ZHEMICYL+X $0110.9537548 .181-19.129$

ZHEMICYL+X $2111.2458549 .451-19.764$ CUBOID $31105.5675030 .48-30.4849 .451$

$-35.004$

HOLE 2105.567500

HOLE 352.7837530 .480

HOLE $452.78375-30.480$

UNIT 2

ZHEMICYL-X 116.350 2P19.05

ZHEMICYL-X $216.4292 P 19.129$

ZHEMICYL-X $0110.9537548 .181-19.129$

ZHEMICYL-X $2111.2458549 .451-19.764$

UNIT 3

ZHEMICYL-Y $116.3502 P 19.05$

ZHEMICYL-Y 216.429 2P19.129

ZHEMICYL-Y 0110.9537548 .181 -19.129

ZHEMICYL-Y $2111.2458549 .451-19.764$

UNIT 4

ZHEMICYL+Y $116.3502 \mathrm{P} 19.05$

ZHEMICYL+Y $216.4292 P 19.129$

ZHEMICYL+YO $110.9537548 .181-19.129$

ZHEMICYL+Y $2111.2458549 .451-19.764$

END GEOM

READ BNDS XFC=SPEC YFC=SPEC END BNDS

READ PLOT TTL=!UO2 10 FULL D D INF!

$P L T=Y E S$ PIC $=$ MIXTURE XŨL $=0$ YUL $=35 \mathrm{ZUL}=0$

$X L R=110 \quad Y L R=-35 \quad Z L R=0$

$U A X=1 \quad V D N=-1 \quad N A X=130 \quad L P \mid=8$

$\mathrm{NCH}=$ ! UF.W IEND PI_OT

END DATA

END

=CSAS25

UO2 10 FULL IN D

27GR̄OUPNDF̄ INFHOMMEDIUM

U-234 $10.08 .1100-5$

U-235 $10.05 .254-3$

U-238 $10.04 .51-5$

H $10.05 .3804-2$

$010.03 .7663-2$

CARBONSTEEL 21.0

AL 3 0.0 5.54-4

CA $30.05 .30-3$

C $30.06 .59-3$

H 30.0 .0133

030.0 .0457

FE $30.03 .59-4$

MG $30.03 .25-3$

SI $30.08 .43-3$

NA $30.03 .89-4$

S $30.01 .09-4$

$\mathrm{H} 2 \mathrm{O} 41.0$

END COMP

UO2 10 FULL W D

READ PATRAM $\bar{R} U \bar{N}=Y$ YES END PARAM

READ GEOM

CYLINDER $116.35019 .05-19.05$

CYLINDER $216.42919 .129-19.129$

CYLINDER $4110.9537548 .181-19.129$

CYLINDER $2111.2458549 .451-19.764$ 
CUBOID $3350 \quad-5050-5049.451-50.549$

END GEOM

READ BNDS XFC=VAC YFC=VAC END BNDS

READ PLOT TTL=IUO2 10 FULL_W_D!

PLT $=$ YES PIC=MIXTURE XÜL $=-5 \overline{0}$ YUTL=00

$Z U L=50$ XLR $=50$ YLR $=00$ ZLR $=-30$

$U A X=1$ WDN $=-1 \quad N A X=130$ LPI $=8$

$\mathrm{NCH}=$ ! UFCW IEND PLOT

END DATA

END

-CSAS25

UO2 15 3.47 D D

27GĀOUPNDF 4 INFHOMMEDIUM

U-234 $10.05 .99-5$

U.235 10.0 .003878

END

U.238 $10.03 .33-5$

END

H 10.0 .059566

010.0 .037725

CARBONSTEEL 21.0

AL $30.05 .54-4$

CA $30.05 .30-3$

C $30.06 .59-3$

H 30.0 .0133

030.0 .0457

FE $30.03 .59-4$

MG $30.03 .25-3$

SI $30.08 .43-3$

NA $30.03 .89-4$

S $30.01 .09-4$

$\mathrm{H} 2 \mathrm{O} 41.0$

END COMP

UO2 $153.47 \mathrm{D} \mathrm{D}$

READ PARAM $\bar{M} \bar{U} N=$ YES END PARAM READ GEOM

CYLINDER $116.350-.9397338-19.05$

CYLINDER $016.35019 .05-19.05$

CYLINDER $216.42919 .129-19.129$

CYLINDER $0110.9537548 .181-19.129$

CYLINDER $2111.2458549 .451-19.764$

CUBOID $3350-5050-5049.451-50.549$

END GEOM

READ BNDS XFC $=V A C$ YFC $=V A C$ END BNDS

READ PLOT TTL =IUO2 15 3.47 D D!

$P L T=Y E S P I C=M I X T U R \bar{E} X \bar{U} L=-50$ YUL $=00$

$Z U L=50$ XLR $=50$ YLR $=00 \quad Z L R=-30$

$U A X=1$ WDN $=-1 \quad N A X=130 \quad L P I=8$

$N C H=! U F C W$ IEND PLOT

END DATA

END

\section{=CSAS25}

UO2 15 3.47 D D INF

27GR̄OUPNDFA INFḦHOMMEDIUM

U-234 $10.05 .99-5$

U-235 10.0 .003878

U-238 $10.03 .33-5$

H 10.0 .059566

010.0 .037725

CARBONSTEEL 21.0

AL $30.05 .54-4$

CA $30.05 .3 C \cdot 3$

C $30.06 .59-3$

H 30.0 .0133
END

END

END

END

END

END

END

END

END

END
O 30.0 .0457

FE $30.03 .59-4$

MG $30.03 .25-3$

END

SI $30.08 .43-3$

NA $30.03 .89-4$

S $30.01 .09-4$

END

$\mathrm{H} 2 \mathrm{O} 41.0$

END COMP

UO2 $15 \quad 3.47$ D D INF

READ GEOM

UNIT 1

ZHEMICYL+X $116.350-9397338$-19.05

ZHEMICYL+X $016.3502 P 19.05$

ZHEMICYL+X 216.429 2P19.129

ZHEMICYL $+X 0110.9537548 .181-19.129$

ZHEMICYL+X $2111.2458549 .451-19.764$

CUBOID $31105.5675030 .48-30.4849 .451$

$-35.004$

HOLE 2105.567500

HOLE 352.7837530 .480

HOLE $452.78375-30.480$

UNIT 2

ZHEMICYL-X $116.350-.9397338$-19.05

ZHEMICYL-X 016.350 2P19.05

ZHEMICYL-X 216.429 2P19.129

ZHEMICYL-X 0110.95375 48.181 -19.129

ZHEMICYL-X $2111.2458549 .451-19.764$

UNIT 3

ZHEMICYL-Y $116.350-.9397338$-19.05

ZHEMICYL-Y 016.350 2P19.05

ZHEMICYL-Y 216.429 2P19.129

ZHEMICYL-Y 0110.9537548 .181 -19.129

ZHEMICYL-Y $2111.2458549 .451-19.764$

UNIT 4

ZHEMICYL+Y $116.350-.9397338$-19.05

ZHEMICYL+Y $016.3502 P 19.05$

ZHEMICYL+Y 216.429 2P19.129

ZHEMICYL+Y $0110.9537548 .181-19.129$

ZHEMICYL+Y $2111.2458549 .451-19.764$

END GEOM

READ BNDS XFC $-S P E C$ YFC-SPEC END BNDS

READ PLOT TTL $=! U O 2 \quad 153.47$ D D INF!

PLT $=Y E S P I C=M I X T U R E X \bar{U} L=0 \bar{Y} U \bar{L}=\overline{35} Z U L=0$

$X L R=110$ YLR $=-35$ ZLR $=0$

$U A X=1$ VDN $=-1 \quad N A X=130$ LPI $=8$

$\mathrm{NCH}=\mid$ UF.W $\mid$ END PLOT

END DATA

END

\section{=CSAS25}

UO2_15_3.47_W_D

27GR̄OUPNDF 4 INFHOMMEDIUM

U-234 $10.05 .99-5$

U-235 10.0 .003878

U-238 $10.03 .33-5$

END

H 10.0 .059566

010.0 .037725

CARBONSTEEL 21.0

AL $30.05 .54-4$

CA $30.05 .30-3$

C $30.06 .59-3$

H 30.0 .0133

O 30.0 .0457

F $30.03 .59-4$

END

END

END

END

END

END

END

END

END

END

END 


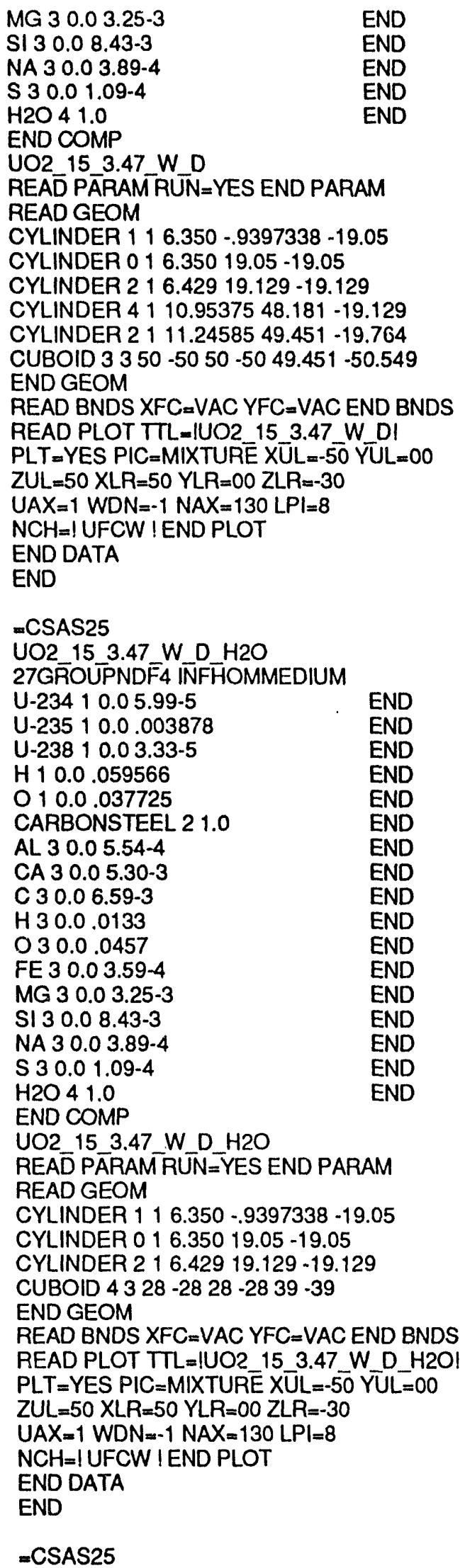

UO2_15_3.47_W_D_INF 27GR̄OUPNDF4 INFF̈OMMEDIUM

U-234 $10.05 .99-5$

U-235 10.0 .003878

U-238 $10.03 .33-5$ END

H1 0.0 .059566 END

$010.0 .037725 \quad$ END

CARBONSTEEL 21.0 END

AL 3 0.0 5.54-4 END

CA $30.05 .30-3$ END

C $30.06 .59-3$ END

H3 0.0 .0133 END

O 30.0 .0457 END

FE $30.03 .59-4 \quad$ END

MG $30.03 .25-3 \quad$ END

SI $30.08 .43-3 \quad$ END

NA $30.03 .89 .4 \quad$ END

S $30.01 .09-4$ END

H2O 41.0 END

END COMP

UO2 _15_3.47_W_D_INF

READ GËOM

UNIT 1

ZHEMICYL+X $116.350-9397338$-19.05

$Z H E M I C Y L+X 016.3502 P 19.05$

ZHEMICYL+X $216.4292 P 19.129$

ZHEMICYL+X $4110.9537548 .181-19.129$

ZHEMICYL+X $2111.2458549 .451-19.764$

CUBOID $31105.5675030 .48-30.4849 .451$

$-35.004$

HOLE 2105.567500

HOLE 352.7837530 .480

HOLE $452.78375-30.480$

UNIT 2

ZHEMICYL-X $116.350-9397338-19.05$

ZHEMICYL-X $016.3502 P 19.05$

ZHEMICYL-X $216.4292 P 19.129$

ZHEMICYL-X $4110.9537548 .181-19.129$

ZHEMICYL-X $2111.2458549 .451-19.764$

UNIT 3

ZHEMICYL-Y $116.350-.9397338$-19.05

ZHEMICYL-Y 016.350 2P19.05

ZHEMICYL-Y 216.429 2P19.129

ZHEMICYL-Y $4110.9537548 .181-19.129$

ZHEMICYL-Y $2111.2458549 .451-19.764$

UNIT 4

ZHEMICYL+Y $116.350-.9397338-19.05$

$Z H E M I C Y L+Y 016.3502 P 19.05$

ZHEMICYL+Y $216.4292 P 19.129$

ZHEMICYL+Y 4110.9537548 .181 -13.129

ZHEMICYL+Y $2111.2458549 .451-19.764$

END GEOM

READ BNDS XFC $=$ SPEC YFC $=$ SPEC END BNDS

READ PL.OT TTL=IUO2 $153.47 \mathrm{~W} D$ INF

PLT $=Y E S$ PIC $=M I X T U R \bar{E} X \bar{U} L=0 \bar{Y} U \bar{L}=\overline{35} Z U L=0$

$X L R=110$ YLR $=-35$ ZLR $=0$

$U A X=1$ VDN $=-1 \quad N A X=130 \quad L P I=8$

$\mathrm{NCH}=$ !UF.W ! END PLOT

END DATA

END

$=$ CSAS25

UO2 15 3.47 W W INF

27GR̄OUPNDF4 INFHOMMEDIUM 


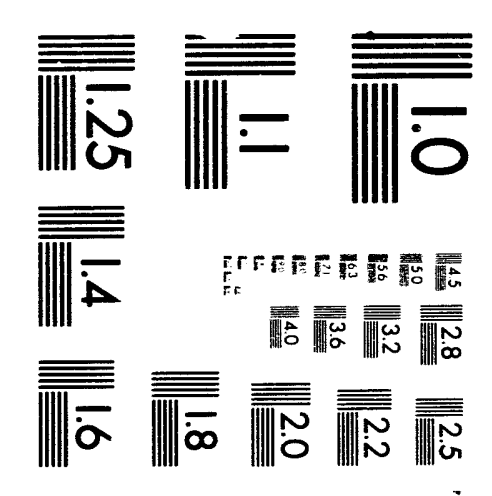



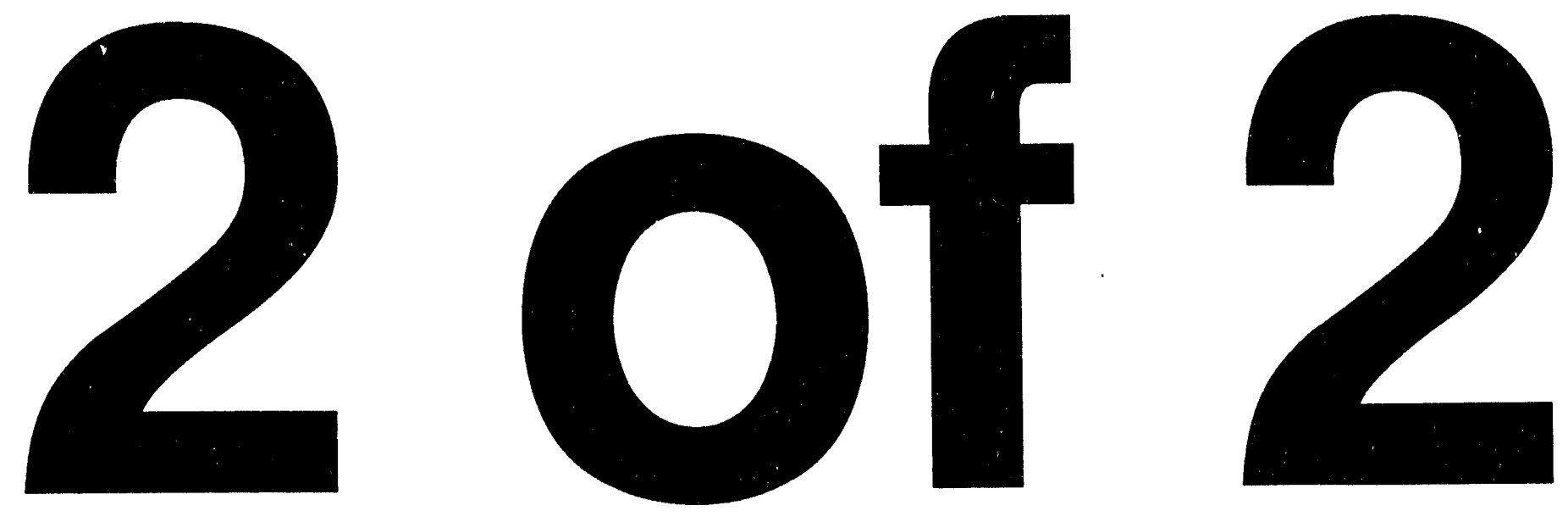


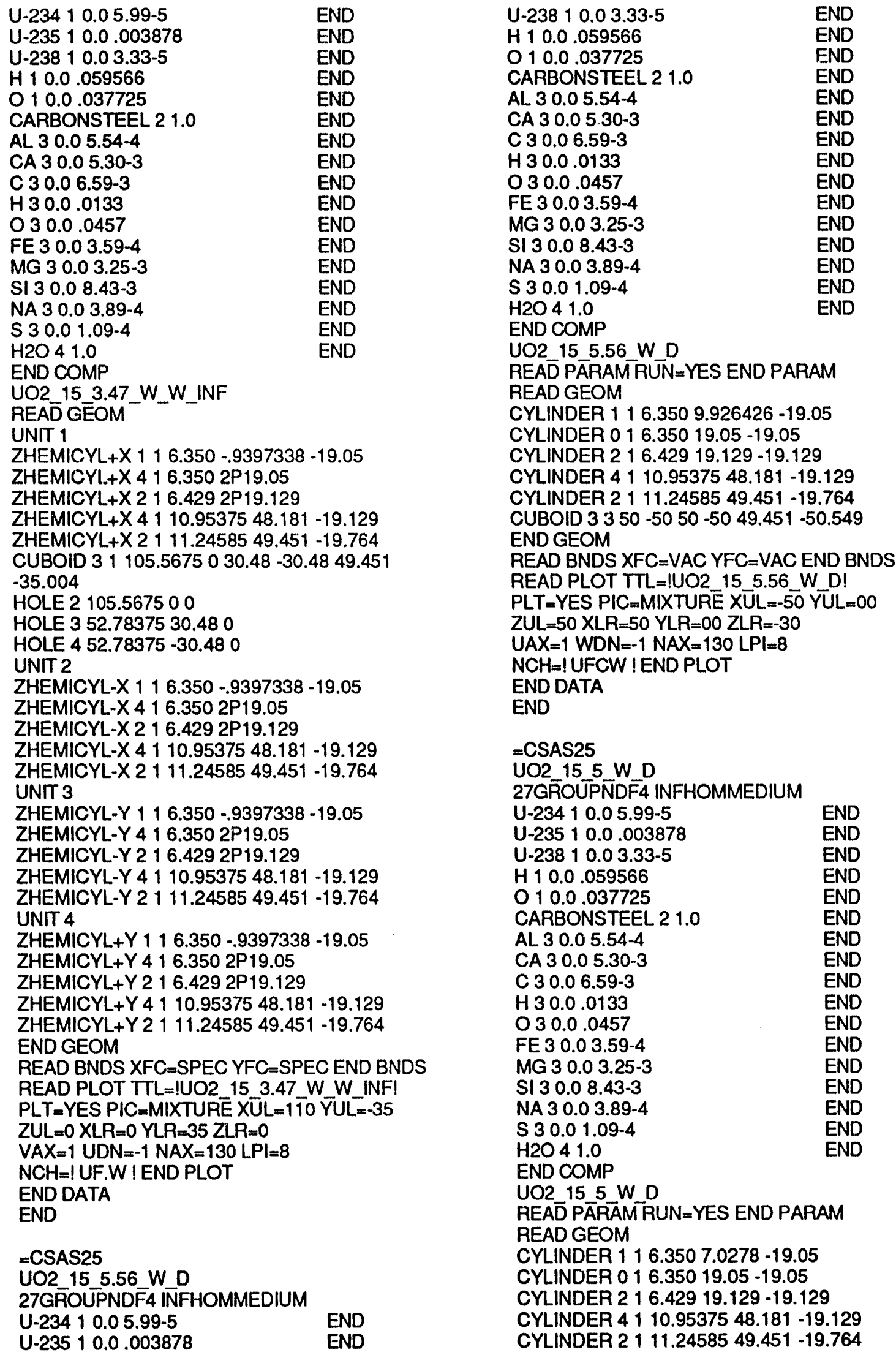


CUBOID $3350-5050-5049.451-50.549$

END GEOM

READ BNDS XFC $=V A C$ YFC $=V A C$ END BNDS

READ PLOT TTL=IUO2 $155 \mathrm{~W}$ D!

PLT $=$ YES PIC $=$ MIXTURE XUL $=-50$ YUL $=00$

$Z U L=50 X L R=50$ YLR $=00$ ZLR $=-30$

$U A X=1$ WDN $=-1 \quad N A X=130 L P \mid=8$

$\mathrm{NCH}=$ ! UFCW ! END PLOT

END DATA

END

-CSAS25

UO2 15 5 W M 5

27GROOUPNDF4 INFHOMMEDIUM

U-234 $10.05 .0228 E-05$

U-235 $10.03 .2541 E-03$

U-238 $10.02 .7959 E-05$

END

H $10.06 .0742 \mathrm{E}-02$

O $10.03 .7036 E-02$

CARBONSTEEL 21.0

AL $30.05 .54-4$

CA $30.05 .30-3$

C $30.06 .59-3$

H 30.0 .0133

030.0 .0457

FE $30.03 .59-4$

MG $30.03 .25-3$

SI $30.08 .43-3$

NA $30.03 .89-4$

$S 30.01 .09-4$

$\mathrm{H} 2 \mathrm{O} 41.0$

END COMP

UO2 155 W M 5

REA $\bar{D}$ PĀRAM $\bar{R} \bar{U} \bar{N}=$ YES END PARAM

READ GEOM

CYLINDER $116.35012 .0278-19.05$

CYLINDER $016.35019 .05-19.05$

CYLINDER $216.42919 .129-19.129$

CYLINDER $4110.9537548 .181-19.129$

CYLINDER $2111.2458549 .451-19.764$

CUBOID $3350-5050-5049.451-50.549$

END GEOM

READ BNDS XFC=VAC YFC $=V A C$ END BNDS

READ PLOT TTL =!UO2_15_5_W_M 5!

$P L T=Y E S$ PIC $=$ MIXTURE XUL $=-50$ YUL $=00$

$Z U L=50$ XLR $=50$ YLR $=00$ ZLR $=-30$

$U A X=1 W D N=-1 N A X=130 L P I=8$

$\mathrm{NCH}=|\mathrm{UFCW}| \mathrm{END}$ PLOT

END DATA

END

$=$ CSAS25

UO2 155 W M 7

27GROUPNDFA INFHOMMEDIUM

U-234 10.0 4.7191E-05

U-235 $10.03 .0574 E-03$

$\mathrm{U}-23810.02 .6268 \mathrm{E}-05$

$\mathrm{H} 10.06 .1112 \mathrm{E}-02$

O $10.03 .6818 E-02$

CARBONSTEEL 21.0

AL 3 0.0 5.54-4

CA $30.05 .30-3$

C $30.06 .59-3$

H 30.0 .0133
030.0 .0457

END

FE $30.03 .59-4$

END

MG $30.03 .25-3$

SI $30.08 .43-3$

NA $30.03 .89-4$

S $30.01 .09-4$

$\mathrm{H} 2 \mathrm{O} 41.0$

END COMP

UO2 155 W M 7

READ PARAM RŪN=YES END PARAM

READ GEOM

CYLINDER $116.35014 .0278-19.05$

CYLINDER $016.35019 .05-19.05$

CYLINDER $216.42919 .129-19.129$

CYLINDER $4110.9537548 .181-19.129$

CYLINDER $2111.2458549 .451-19.764$

CUBOID $3350-5050-50 \quad 49.451-50.549$

END GEOM

READ BNDS XFC $=V A C$ YFC $=V A C$ END BNDS

READ PLOT TTL $=$ !UO2 $155 \mathrm{~W} M 7 !$

PLT $=Y E S$ PIC $=$ MIXTURE $X \bar{U} \bar{L}=-5 \bar{Y}$ YUL $=00$

$Z U L=50 X L R=50$ YLR $=00 Z L R=-30$

$U A X=1$ WDN $=-1 \quad N A X=130 L P I=8$

$\mathrm{NCH}=$ ! UFCW ! END PLOT

END DATA

END

\section{$=$ CSAS25}

UO2 $155 \mathrm{~W} M 10$

27GR̄OUPNDF4 INFHOMMEDIUM

U-234 10.0 4.3267E-05

U-235 $10.02 .8031 E-03$

U-238 $10.02 .4084 E-05$

H 10.06 .1590 E-02

O $10.03 .6536 E-02$

CARBONSTEEL 21.0

AL $30.05 .54-4$

CA $30.05 .30-3$

C $30.06 .59-3$

H3 0.0 .0133

O 30.0 .0457

FE $30.03 .59-4$

MG $30.03 .25-3$

SI $30.08 .43-3$

NA $30.03 .89-4$

S $30.01 .09-4$

$\mathrm{H} 2 \mathrm{O} 41.0$

END COMP

UO2 155 W M 10

REA $\bar{D}$ PATAM $\bar{R} \bar{U} \bar{N}=$ YES END PARAM

READ GEOM

CYLINDER $116.35017 .0278-19.05$

CYLINDER $016.35019 .05-19.05$

CYLINDER $216.42919 .129-19.129$

CYLINDER $4110.9537548 .181-19.129$

CYLINDER 2111.2458549 .451 -19.764

CUBOID $3350-5050-5049.451-50.549$

END GEOM

READ BNDS XFC $=V A C$ YFC $=$ VAC END BNDS

READ PLOT TTL=!UO2_15_5_W_M_10!

PLT $=$ YES PIC $=M I X T U R \bar{E}$ XU $\bar{L}=-5 \overline{0}$ YUL $=00$

$Z U L=50$ XLR $=50$ YLR $=00$ ZLR $=-30$

$U A X=1$ WDN -1 NAX $=130 \mathrm{LPI}=8$

$\mathrm{NCH}=$ ! UFCW IEND PLOT 


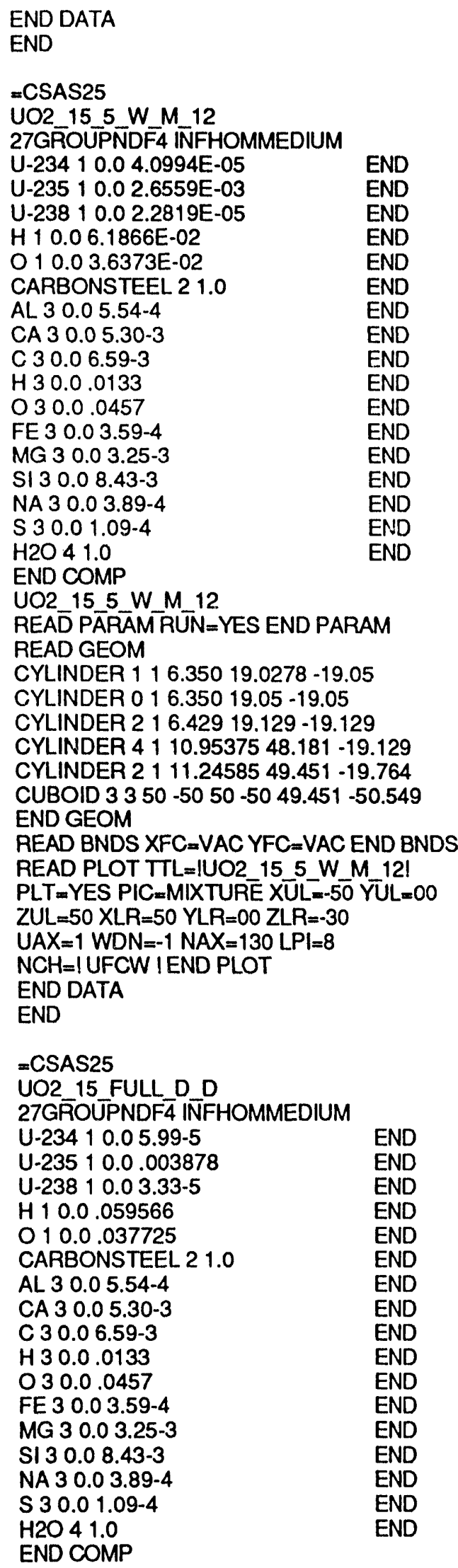

UO2_15_FULL_D_D

REA $\bar{D}$ PARAM $\bar{R} U \bar{N}=$ YES END PARAM READ GEOM

CYLINDER 116.35019 .05 -19.05

CYLINDER $216.42919 .129-19.129$

CYLINDER $0110.9537548 .181-19.129$

CYLINDER $2111.2458549 .451-19.764$

CUBOID $3350-5050-50 \quad 49.451-50.549$

END GEOM

READ BNDS XFC $=V A C$ YFC $=V A C$ END BNDS

READ PLOT TTL $=$ !UO2 15 FULL D D!

PLT $=Y E S$ PIC $=M I X T U R E$ XUL $=-50$ YUL $=00$

$Z U L=50 X L R=50$ YLR $=00 \quad Z L R=-30$

$U A X=1$ WDN $=-1 \quad N A X=130 \quad L P I=8$

$\mathrm{NCH}=$ ! UFCW ! END PLOT

END DATA

END

$=$ CSAS 25

UO2 15 FULL_D_D INF 27GR̄OUPNDFA INFHOMMEDIUM

U-234 $10.05 .99-5$

U-235 10.0 .003878

U-238 $10.03 .33-5$

H 10.0 .059566

010.0 .037725

AL 3 0.0 5.54-4

CA $30.05 .30-3$

C $30.06 .59-3$

H 30.0 .0133

030.0 .0457

FE $30.03 .59-4$

MG $30.03 .25-3$

SI $30.08 .43-3$

NA $30.03 .89-4$

S $30.01 .09-4$

$\mathrm{H} 2 \mathrm{O} 41.0$

END COMP

UO2_15_FULL_D_D_INF

READ GEOM

UNIT 1

ZHEMICYL+X $116.3502 P 19.05$

ZHEMICYL+X $216.4292 P 19.129$

ZHEMICYL+X $0110.9537548 .181-19.129$

ZHEMICYL+X $2111.2458549 .451-19.764$

CUBOID $31105.5675030 .48-30.4849 .451$

$-35.004$

HOLE 2105.567500

HOLE 352.7837530 .480

HOLE $452.78375-30.480$

UNIT 2

ZHEMICYL-X 116.350 2P19.05

ZHEMICYL-X 216.429 2P19.129

ZHEMICYL-X $0110.9537548 .181-19.129$

ZHEMICYL-X $2111.2458549 .451-19.764$

UNIT 3

ZHEMICYL-Y 116.350 2P19.05

ZHEMICYL-Y 216.429 2P19.129

ZHEMICYL-Y $0110.9537548 .181-19.129$

ZHEMICYL-Y $2111.2458549 .451-19.764$

UNIT 4

ZHEMICYL+Y $116.3502 P 19.05$

ZHEMICYL+Y $216.4292 P 19.129$ 
ZHEMICYL+YO $110.9537548 .181-19.129$ ZHEMICYL+Y $2111.2458549 .451-19.764$ END GEOM

READ BNDS XFC=SPEC YFC=SPEC END BNDS READ PLOT TTL=IUO2 15 FULL $D$ D INFI $P L T=Y E S$ PIC $=M I X T U R \bar{E} X \bar{U} L=0$ YUL $-3 \overline{5}$ ZUL $=0$ $X L R=110$ YLR $=-35$ ZLR $=0$

$U A X=1$ VDN $=-1 \quad N A X=130 \quad L P I=8$

$\mathrm{NCH}=$ !UF.W ! END PLOT

END DATA

END

\section{$=$ CSAS 25}

UO2 15 FULL W D

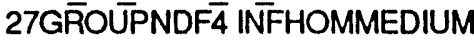

U-234 $10.05 .99-5$

U-235 10.0 .003878

END

$\mathrm{U}-23810.03 .33-5$

H 10.0 .059566

O 10.0 .037725

CARBONSTEEL 21.0

AL $30.05 .54-4$

CA $30.05 .30-3$

C $30.06 .59-3$

H 3 C.0.0133

O 30.0 .0457

FE $30.03 .59-4$

MG $30.03 .25-3$

SI $30.08 .43-3$

NA $30.03 .89-4$

$S 30.01 .09-4$

$\mathrm{H} 2 \mathrm{O} 41.0$

END COMP

UO2 15 FULL W D

REA $\bar{D}$ PĀRAM $\bar{R} U \bar{N}=Y E S$ END PARAM

READ GEOM

CYLINDER $116.35019 .05-19.05$

CYLINDER $216.42919 .129-19.129$

CYLINDER $4110.9537548 .181-19.129$

CYLINDER $2111.2458549 .451-19.764$

CUBOID $3350-5050-5049.451-50.549$

END GEOM

READ BNDS XFC=VAC YFC $=V A C$ END BNDS

READ PLOT TTL=!UO2_15_FULL_W_D!

$P L T=Y E S$ PIC $=$ MIXTURE $\overline{X U L}=-5 \overline{0}$ YŨL $=00$

$Z U L=50 X L R=50 Y L R=00 \quad Z L R=-30$

$U A X=1 \quad W D N=-1 \quad N A X=130 \quad L P \mid=8$

$\mathrm{NCH}=$ ! UFCW ! END PLOT

END DATA

END

\section{$=$ CSAS 25}

UO2 651.39 D D

27GR $\bar{R}$ OUPNDF4 INFHOMMEDIUM

U-234 $10.01 .540-5$

U-235 10.0 .001001

U-238 $10.08 .56-6$

H 10.0 .06661

010.0 .035354

CARBONSTEEL 21.0

AL $30.05 .54-4$

CA $30.05 .30-3$

C $30.06 .59-3$

H 30.0 .0133
030.0 .0457

END

FE $30.03 .59-4$

END

MG $30.03 .25-3$

SI $30.08 .43-3$

NA $30.03 .89-4$

S $30.01 .09-4$

$\mathrm{H} 2 \mathrm{O} 41.0$

END COMP

UO2 $65 \quad 1.39 \mathrm{D} D$

READ PARAM $\bar{R} \bar{U} N=Y E S$ END PARAM

READ GEOM

CYLINDER $116.3509 .020928-19.05$

CYLINDER $016.35019 .05-19.05$

CYLINDER $216.42919 .129-19.129$

CYLINDER $0110.9537548 .181-19.129$

CYLINDER $2111.2458549 .451-19.764$

CUBOID $3350-5050-5049.451-50.549$

END GEOM

READ BNDS XFC=VAC YFC=VAC END BNDS

READ PLOT TTL $=! U O 2 \quad 65 \quad 1.39 \mathrm{D} D$ !

$P L T=Y E S P I C=M I X T U R \bar{E} X \bar{U} L=-50$ YUL $=00$

$Z U L=50$ XLR $=50$ YLR $=00$ ZLR $=-30$

$U A X=1$ WDN $=-1 \quad N A X=130 \quad L P \mid=8$

$\mathrm{NCH}=$ ! UFCW ! END PLOT

END DATA

END

$=$ CSAS25

UO2 651.39 D D H2O

27GROUPNDF4 INFHOMMEDIUM

U-234 $10.01 .540-5$

U-235 10.0 .001001

U-2381 0.0 8.56-6

H 10.0 .06661

O 10.0 .035354

CARBONSTEEL 21.0

AL $30.05 .54-4$

CA $30.05 .30-3$

C $30.06 .59-3$

H 30.0 .0133

030.0 .0457

FE $30.03 .59-4$

MG $30.03 .25-3$

SI $30.08 .43-3$

NA $30.03 .89-4$

S $30.01 .09-4$

$\mathrm{H} 2 \mathrm{O} 41.0$

END COMP

UO2 651.39 D D H2O

READ PARAM RUUN=YES END PARAM

READ GEOM

CYLINDER $116.3509 .020928-19.05$

CYLINDER $016.35019 .05-19.05$

CYLINDER $216.42919 .129-19.129$

CUBOID $4228-2828-2839-39$

END GEOM

READ BNDS XFC=VAC YFC $=V A C$ END BNDS

READ PLOT TTL=IUO2 65 1.39 D D H2O!

$P L T=Y E S$ PIC $=$ MIXTURE XU $\bar{L}=-50$ YUL $=00$

$Z U L=50 \quad X L R=50 \quad Y L R=00 \quad Z L R=-30$

$U A X=1$ WDN $=-1 \quad N A X=130 \mathrm{LPl}=8$

$\mathrm{NCH}=$ I UFCW $\mid$ END PLOT

END DATA

END 
$=$ CSAS25

UO2 651.39 D D INF

27GROUPNDF4 INFHOMMEDIUM

U-234 $10.01 .540-5$

U-235 10.0 .001001

END

U-238 $10.08 .56-6$

H 10.0 .06661

O 10.0 .035354

CARBONSTEEL 21.0

AL 3 0.0 5.54-4

CA $30.05 .30-3$

C $30.06 .59-3$

H3 0.0 .0133

O 30.0 .0457

FE $30.03 .59-4$

MG $30.03 .25-3$

SI $30.08 .43-3$

NA $30.03 .89-4$

S $30.01 .09-4$

$\mathrm{H} 2 \mathrm{O} 41.0$

END COMP

UO2 65_1.39_D_D_INF

READ̄ GEOM

UNIT 1

ZHEMICYL+X $116.3509 .020928-19.05$

ZHEMICYL+X $016.3502 P 19.05$

ZHEMICYL+X $216.4292 P 19.129$

ZHEMICYL+X $0110.9537548 .181-19.129$

ZHEMICYL+X $2111.2458549 .451-19.764$

CUBOID $31105.5675030 .48-30.4849 .451$ $-35.004$

HOLE 2105.567500

HOLE 352.7837530 .480

HOLE $452.78375-30.480$

UNIT 2

ZHEMICYL-X 116.3509 .020928 -19.05

ZHEMICYL-X $016.3502 P 19.05$

ZHEMICYL-X 216.429 2P19.129

ZHEMICYL-X $0110.9537548 .181-19.129$

ZHEMICYL-X 2111.2458549 .451 -19.764

UNIT 3

ZHEMICYL-Y 116.3509 .020928 -19.05

ZHEMICYL-Y 016.350 2P19.05

ZHEMICYL-Y 216.429 2P19.129

ZHEMICYL-Y $0110.9537548 .181-19.129$

ZHEMICYL-Y 2111.2458549 .451 -19.764

UNIT 4

ZHEMICYL+Y 116.3509 .020928 -19.05

ZHEMICYL+Y $016.3502 P 19.05$

ZHEMICYL+Y $216.4292 P 19.129$

ZHEMICYL+Y $0110.9537548 .181-19.129$

ZHEMICYL+Y $2111.2458549 .451-19.764$

END GEOM

READ BNDS XFC $=S P E C$ YFC $=S P E C$ END BNDS

READ PLOT TTL $=\mid U 0265 \quad 1.39 \quad D \quad D$ INF!

$P L T=Y E S$ PIC $=M I X T U R \bar{E} X \overline{U L}=0 \bar{Y} U \bar{L}=\overline{3} 5 \mathrm{ZUL}=0$

$X L R=110 \quad Y L R=-35 \quad Z L R=0$

$U A X=1$ VDN $=-1 \quad N A X=130 \quad L P I=8$

$\mathrm{NCH}=!$ UF.W $\mid$ END PLOT

END DATA

END

=CSAS25
UO2 651.39 W D

27GR̄OUPNDF4 INTHOMMEDIUM

U-234 $10.01 .540-5$

U-235 10.0 .001001

U-238 $10.08 .56-6$

H 10.0 .06661

O 10.0 .035354

CARBONSTEEL 21.0

AL 3 0.0 5.54-4

CA $30.05 .30-3$

C $30.06 .59-3$

H 30.0 .0133

O 30.0 .0457

FE $30.03 .59-4$

MG $30.03 .25-3$

SI $30.08 .43-3$

NA $30.03 .89-4$

S $30.01 .09-4$

$\mathrm{H} 2 \mathrm{O} 41.0$

END COMP

UO2 $65 \quad 1.39$ W D

READ PARAM RUNN=YES END PARAM

READ GEOM

CYLINDER $116.3509 .020928-19.05$

CYLINDER $016.35019 .05-19.05$

CYLINDER $216.42919 .129-19.129$

CYLINDER $4110.9537548 .181-19.129$

CYLINDER $2111.2458549 .451-19.764$

CUBOID $3350-5050-5049.451-50.549$

END GEOM

READ BNDS XFC $=V A C$ YFC $=V A C$ ENU BNDS

READ PLOT $T T=$ !UO2 $651.39 \mathrm{~W} \mathrm{D}$ !

PLT $=$ YES PIC $=$ MIXTURE XUL $=-50$ YUL $=00$

$Z U L=50 \quad X L R=50$ YLR $=00 \quad Z L R=-30$

$U A X=1$ WDN $=-1 \quad N A X=130 \quad L P I=8$

$\mathrm{NCH}=$ ! UFCW !END PLOT

END DATA

END

\section{=CSAS25}

UO2_65_1.39_W_D_INF 27GR̄OUPNDF4 INTFHOMMEDIUM

U-234 $10.01 .540-5$

U-235 10.0 .001001

U-238 $10.08 .56-6$

H 10.0 .06661

010.0 .035354

CARBONSTEEL 21.0

AL 3 0.0 5.54-4

CA $30.05 .30-3$

C $30.06 .59-3$

H3 0.0 .0133

O 30.0 .0457

FE $30.03 .59-4$

MG $30.03 .25-3$

SI $30.08 .43-3$

NA $30.03 .89-4$

S $30.01 .09-4$

$\mathrm{H} 2 \mathrm{O} 41.0$

END COMP

UO2_65_1.39_W_D_INF

READ GEOM

UNTT 1

ZHEMICYL+X $116.3509 .020928-19.05$ 
ZHEMICYL+X $016.3502 P 19.05$

ZHEMICYL+X $216.4292 P 19.129$

ZHEMICYL+X $4110.9537548 .181-19.129$

ZHEMICYL+X $2111.2458549 .451-19.764$

CUBOID $31105.5675030 .48-30.4849 .451$

$-35.004$

HOLE 2105.567500

HOLE 352.7837530 .480

HOLE $452.78375-30.480$

UNIT 2

ZHEMICYL-X 116.3509 .020928 -19.05

ZHEMICYL-X $016.3502 P 19.05$

ZHEMICYL-X 216.429 2P19.129

ZHEMICYL-X $4110.9537548 .181-19.129$

ZHEMICYL-X 2111.2458549 .451 -19.764

UNIT 3

ZHEMICYL-Y 116.3509 .020928 -19.05

ZHEMICYL-Y 016.350 2P19.05

ZHEMICYL-Y 216.429 2P19.129

ZHEMICYL-Y $4110.9537548 .181-19.129$

ZHEMICYL-Y $2111.2458549 .451-19.764$

UNIT 4

ZHEMICYL+Y 116.3509 .020928 -19.05

ZHEMICYL+YO $16.3502 P 19.05$

ZHEMICYL+Y $216.4292 P 19.129$

ZHEMICYL+Y $4110.9537548 .181-19.129$

ZHEMICYL+Y $2111.2458549 .451-19.764$

END GEOM

READ BNDS XFC=SPEC YFC=SPEC END BNDS

READ PLOT TTL=!UO2 65 1.39 W D INF!

$P L T=Y E S P I C=M I X T U R E \bar{X}$ U $=0 \bar{Y} U \bar{L}=35 \mathrm{ZUL}=0$

$X L R=110$ YLR $=-35 Z L R=0$

$U A X=1 \quad V D N=-1 \quad N A X=130 \quad L P I=8$

$\mathrm{NCH}=$ ! UF.W $\mathrm{W}$ ! END PLOT

END DATA

END

\section{CSAS25}

UU2 65_1_W_D

27GR̄OUPND $\bar{F} 4$ INFHOMMEDIUM

U-234 $10.01 .540-5$

U-235 10.0 .001001

U-238 $10.08 .56-6$

H 10.0 .06661

O 10.0 .035354

CARBONSTEEL 21.0

AL 3 0.0 5.54-4

CA $30.05 .30-3$

C $30.06 .59-3$

H3 0.0 .0133

O 30.0 .0457

FE $30.03 .59-4$

MG $30.03 .25-3$

SI $30.08 .43-3$

NA $30.03 .89-4$

END

END

END

END

END

END

END

END

END

END

END

END

END

END

END

S $30.01 .09-4$

END

$\mathrm{H} 2 \mathrm{O} 41.0$

END

END COMP

UO2 651 W D

READ PARAM $\bar{R} U N=$ YES END PARAM

READ GEOM

CYLINDER $116.3501 .1603-19.05$

CYLINDER $016.35019 .05-19.05$

CYLINDER $216.42919 .129-19.129$
CYLINDER $4110.9537548 .181-19.129$

CYLINDER $2111.2458549 .451-19.764$

CUBOID $3350-5050-5049.451-50.549$

END GEOM

READ BNDS XFC $=$ VAC YFC $=V A C$ END BNDS

READ PLOT TTL=IUO2 $651 \mathrm{~W}$ D!

PLT $=$ YES PIC $=$ MIXTURE XUL $=-50$ YUL $=00$

$Z U L=50$ XLR $=50$ YLR $=00$ ZLR $=-30$

$U A X=1$ WDN $=-1 \quad N A X=130 \quad L P I=8$

$\mathrm{NCH}=$ ! UFCW ! END PLOT

END DATA

END

=CSAS25

UO2 651 W M 5

27GR̄OUPNDD $\overline{4} 4 \bar{N} F H O M M E D I U M$

U-234 $10.01 .3112 \mathrm{E}-05$

U-235 $10.08 .4948 E-04$

U-238 $10.07 .2985 E-06$

H $10.06 .6652 \mathrm{E}-02$

O 1 0.0 3.5066E-02

CARBONSTEEL 21.0

AL 3 0.0 5.54-4

CA $30.05 .30-3$

C $30.06 .59-3$

H 30.0 .0133

030.0 .0457

FE $30.03 .59-4$

MG $30.03 .25-3$

SI $30.08 .43-3$

NA $30.03 .89-4$

S $30.01 .09-4$

$\mathrm{H} 2 \mathrm{O} 41.0$

END COMP

UO2 651 W M 5

READ PATAMM $\overline{\text { R }}$ =YES END PARAM

READ GEOM

CYLINDER $116.35014 .0199-19.05$

CYLINDER $016.35019 .05-19.05$

CYLINDER $216.42919 .129-19.129$

CYLINDER $4110.9537548 .181-19.129$

CYLINDER $2111.2458549 .451-19.764$

CUBOID $3350-5050-5049.451-50.549$

END GEOM

READ BNDS XFC $=V A C$ YFC $=V A C$ END BNDS

READ PLOT TTL $=! U O 2651 \mathrm{~W} M 5$ !

PLT $=$ YES PIC $=$ MIXTURE XUL $=-50$ YUL $=00$

$Z U L=50 X L R=50 \quad Y L R=00 \quad Z L R=-30$

$U A X=1 W D N=-1 \quad N A X=130 L P I=8$

$\mathrm{NCH}=$ ! UFCW ! END PLOT

END DATA

END

\section{$=$ CSAS25}

UO2 65_ 1 W M 10

27GROUPNDEF4 INFHOMMEDIUM

U-234 10.0 1.0334E-05

U-235 $10.06 .6952 E-04$

U-238 10.0 5.7523E-06

H $10.06 .6696 \mathrm{E}-02$

O $10.03 .4719 E-02$

CARBONSTEEL 21.0

AL $30.05 .54-4$

CA $30.05 .30-3$

END

END

END

END

END

END

END

END 
C $30.06 .59-3$

H 30.0 .0133

O 30.0 .0457

FE $30.03 .59-4$

MG $30.03 .25-3$

SI $30.08 .43-3$

NA $30.03 .89-4$

S $30.01 .09-4$

$\mathrm{H} 2 \mathrm{O} 41.0$

END COMP

UO2 651 W M 10

READ PATAMATUN $=$ YES END PARAM

READ GEOM

CYLINDER $116.35011 .1603-19.05$

CYLINDER $016.35019 .05-19.05$

CYLINDER $216.42919 .129-19.129$

CYLINDER 4110.9537548 .181 -19.129

CYLINDER $2111.2458549 .451-19.764$

CUBOID $3350-5050-5049.451-50.549$

END GEOM

READ BNDS XFC $=V A C$ YFC $=V A C$ END BNDS

READ PLOT TTL=!UO2 65_1_W_M 10 !

PLT $=$ YES PIC $=$ MIXTURE XÚL $=-50$ YUL $=00$

$Z U L=50 \quad X L R=50 \quad Y L R=00 \quad Z L R=-30$

$U A X=1$ WDN $=-1 \quad N A X=130 \quad L P I=8$

$\mathrm{NCH}=$ ! UFCW ! END PLOT

END DATA

END

$=$ CSAS25

UO2 65 1W_M 17.8

27GR̄OUPNDF4 INFHOMMEDIUM

U-234 $10.08 .2135 \mathrm{E}-0$

U-235 $10.05 .3213 E-04$

U-238 $10.04 .5719 E-06$

H $10.06 .6729 \mathrm{E}-02$

010.0 3.4455E-02

CARBONSTEEL 21.0

AL $30.05 .54-4$

CA $30.05 .30-3$

C $30.06 .59-3$

H 30.0 .0133

O 30.0 .0457

FE $30.03 .59-4$

MG $30.03 .25-3$

SI $30.08 .43-3$

NA $30.03 .89-4$

S $30.01 .09-4$

$\mathrm{H} 2 \mathrm{O} 41.0$

END COMP

UO2 65 I W M 17.8

READ PATRAM $\bar{R} \bar{U} \bar{N}=Y E S$ END PARAM

READ GEOM

CYLINDER $116.35018 .9603-19.05$

CYLINDER $016.35019 .05-19.05$

CYLINDER $216.42919 .129-19.129$

CYLINDER $4110.9537548 .181-19.129$

CYLINDER $2111.2458549 .451-19.764$

CUBOID $3350-5050-5049.451-50.549$

END GEOM

READ BNDS XFC $=V A C$ YFC $=V A C$ END BNDS

READ PLOT TTL=!UO2 65 1 W M 17.8!

PLT $=Y E S$ PIC $=M I X T U R \bar{E} X \bar{U} L=-5 \overline{0}$ YUL $=00$

$Z U L=50 \quad X L R=50 \quad Y L R=00 \quad Z L R=-30$
$U A X=1 W D N=-1 \quad N A X=130 L P \mid=8$

$\mathrm{NCH}=$ ! UFCW IEND PLOT

END DATA

END

=CSAS25

UO2 65 FULL_D_D_INF

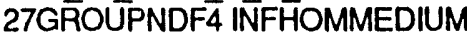

U-234 $10.01 .540-5$

U-235 10.0 .001001

$\mathrm{U}-23810.08 .56-6$

H 10.0 .06661

010.0 .035354

CARBONSTEEL 21.0

AL 30.05 .54 .4

CA $30.05 .30-3$

C $30.06 .59-3$

H 30.0 .0133

030.0 .0457

FE $30.03 .59-4$

MG $30.03 .25-3$

SI $30.08 .43-3$

NA $30.03 .89-4$

S $30.01 .09-4$

$\mathrm{H} 2 \mathrm{O} 41.0$

END COMP

UO2_65_FULL_D_D_INF

READ GEOM

UNIT 1

ZHEMICYL+X 116.350 2P 19.05

ZHEMICYL+X $216.4292 P 19.129$

ZHEMICYL+X $0110.9537548 .181-19.129$

ZHEMICYL+X $2111.2458549 .451-19.764$

CUBOID $31105.5675030 .48-30.4849 .451$

$-35.004$

HOLE 2105.567500

HOLE 352.7837530 .480

HOLE $452.78375-30.480$

UNIT 2

ZHEMICYL-X 116.350 2P19.05

ZHEMICYL-X 216.429 2P19.129

ZHEMICYL-X 0110.95375 48.181 -19.129

ZHEMICYL-X 2111.2458549 .451 -19.764

UNIT 3

ZHEMICYL-Y 116.350 2P19.05

ZHEMICYL-Y 216.429 2P19.129

ZHEMICYL-Y $0110.9537548 .181-19.129$

ZHEMICYL-Y $2111.2458549 .451-19.764$

UNIT 4

ZHEMICYL+Y $116.3502 P 19.05$

ZHEMICYL+\ 216.429 2P19.129

ZHEMICYL+Y $0110.9537548 .181-19.129$

ZHEMICYL+Y2 $111.2458549 .451-19.764$

END GEOM

READ BNDS XFC $=$ SPEC YFC $=S P E C$ END BNDS

READ PLOT TTL $=$ IUO2 65 FULL_D_D INFI

PLT $=Y E S$ PIC $=M I X T U R E \overline{X U L}=0$ YUL $=35 \quad Z U L=0$

$X L R=110 \quad Y L R=-35 \quad Z L R=0$

$U A X=1$ VDN $=-1 \quad N A X=130 \quad L P I=8$

$\mathrm{NCH}=$ ! UF. W ! END PLOT

END DATA

END 


\section{Oxides In 5-inch Canisters, $8 \mathrm{~kg} \mathrm{U}-235$}

$=$ CSAS25

UO2 C $8 \mathrm{~W} \mathrm{M} 0$

27GROUPNDF4 INFHOMMEDIUM

U-234 $10.03 .6785 \mathrm{E}-04$

U-235 $10.02 .3832 E-02$

U-238 $10.02 .0476 E-04$

O $10.04 .8809 E-02$

CARBONSTEEL 21.0

AL $30.05 .54-4$

CA $30.05 .30-3$

C $30.06 .59-3$

H 30.0 .0133

O 30.0 .0457

FE $30.03 .59-4$

MG $30.03 .25-3$

SI $30.08 .43-3$

NA $30.03 .89-4$

S $30.01 .09-4$

$\mathrm{H} 2 \mathrm{O} \quad 41.0$

END COMP

UO2 C 8 W M

READ GEOM

CYLINDER $116.350-1.2260 E+01-19.05$

CYLINDER $416.35019 .05-19.05$

CYLINDER $216.42919 .129-19.129$

CYLINDER $4110.9537548 .181-19.129$

CYLINDER $2111.2458549 .451-19.764$

CUBOID $3350-5050-5049.451-50.549$

END GEOM

READ BNDS $X F C=V A C$ YFC $=V A C$ END BNDS

READ PLOT TTL $=1 U 02 \_C \_8$ W_M_O!

$P L T=Y E S \quad P I C=M I X T U R \bar{E} \overline{X U}=-50 \bar{Y} U L=00$

$Z U L=50 \quad X L R=50$ YLR $=00 \quad Z L R=-30$

$U A X=1$ WDN $=-1$ NAX $=130$ LPI $=8$

$\mathrm{NCH}=$ ! UFCW ! END PLOT

END DATA

END

\section{$=$ CSAS25}

$\mathrm{UO} 2$ C 8 W_M 2

27GROUUPNDF4 INFHOMMEDIUM

U-234 $10.02 .8415 E-04$

U-235 $10.01 .8409 \mathrm{E}-02$

U-238 $10.01 .5817 E-04$

H $10.01 .5213 E-02$

O $10.04 .5310 \mathrm{E}-02$

CARBONSTEEL 21.0

AL $30.05 .54-4$

CA $30.05 .30-3$

C $30.06 .59-3$

H 30.0 .0133

O 30.0 .0457

FE $30.03 .59-4$

MG $30.03 .25-3$

SI $30.08 .43-3$

NA $30.03 .89-4$

$S \quad 30.01 .09-4$

$\mathrm{H} 2 \mathrm{O} 41.0$

END COMP

$\mathrm{UO} 2 \mathrm{C} 8 \mathrm{~W} M 2$

REA $\bar{D} \bar{G} \bar{E} \bar{M}$
CYLINDER $116.350-1.0260 E+01-19.05$

CYLINDER $416.35019 .05-19.05$

CYLINDER $216.42919 .129-19.129$

CYLINDER $4110.9537548 .181-19.129$

CYLINDER $2111.2458549 .451-19.764$

CUBOID $3350-5050-5049.451-50.549$

END GEOM

READ BNDS $X F C=V A C$ YFC $=V A C$ END BNDS

READ PLOT TTL=IUO2 C $8 \mathrm{~W} M 2$ !

$P L T=Y E S$ PIC $=M I X T U R \bar{X} \overline{X U} \bar{L}=-50 \bar{Y} U L=00$

$Z U L=50 \times L R=50$ YLR $=00 \quad Z L R=-30$

$U A X=1$ WDN $=-1 \quad N A X=130 \quad L P I=8$

$\mathrm{NCH}=$ ! UFCW IEND PLOT

END DATA

END

$=$ CSAS25

UO2_C 8 W_M 4

27GROUUPNDF4 INFHOMMEDIUM

U-234 10.02 .3148 E-04

U-235 $10.01 .4997 E-02$

U-238 $10.01 .2885 E-04$

H $10.02 .4787 \mathrm{E}-02$

O $10.04 .3108 E-02$

CARBONSTEEL 21.0

AL $30.05 .54-4$

CA $30.05 .30-3$

C $30.06 .59-3$

H 30.0 .0133

O 30.0 .0457

FE $30.03 .59-4$

MG 30.03.25-3

SI $30.08 .43-3$

NA $30.03 .89-4$

S $30.01 .09-4$

$\mathrm{H} 2 \mathrm{O} \quad 41.0$

END COMP

UO2 C 8 W M 4

READ $\overline{G E O M}$

CYLINDER $116.350-8.2604 E+00-19.05$

CYLINDER $416.35019 .05-19.05$

CYLINDER $216.42919 .129-19.129$

CYLINDER $4110.9537548 .181-19.129$

CYLINDER $2111.2458549 .451-19.764$

CUBOID $3350-5050-5049.451-50.549$

END GEOM

READ BNDS $X F C=V A C$ YFC $=V A C$ END BNDS

READ PLOT TTL $=! U O 2$ C $8 \mathrm{~W} M 4$ !

$P L T=Y E S P I C=M I X T U R \bar{X} \overline{X U}=-50 \bar{Y} U L=00$

$Z U L=50 X L R=50$ YLR $=00$ ZLR $=-30$

$U A X=1$ WDN $=-1 \quad \mathrm{NAX}=130 \mathrm{LPI}=8$

$\mathrm{NCH}=$ ! UFCW ! END PLOT

END DATA

END

\section{$=$ CSAS25}

UO2 C 8 W M 8

27GROUPNDF4 INFHOMMEDIUM

U-234 $10.01 .6887 \mathrm{E}-04$

U-235 $10.01 .0941 \mathrm{E}-02$

U-238 $10.09 .4001 E-05$

H 1 $0.03 .6166 \mathrm{E}-02$

O $10.04 .0490 E-02$

CARBONSTEEL 21.0

END

END

END

END

END

END 


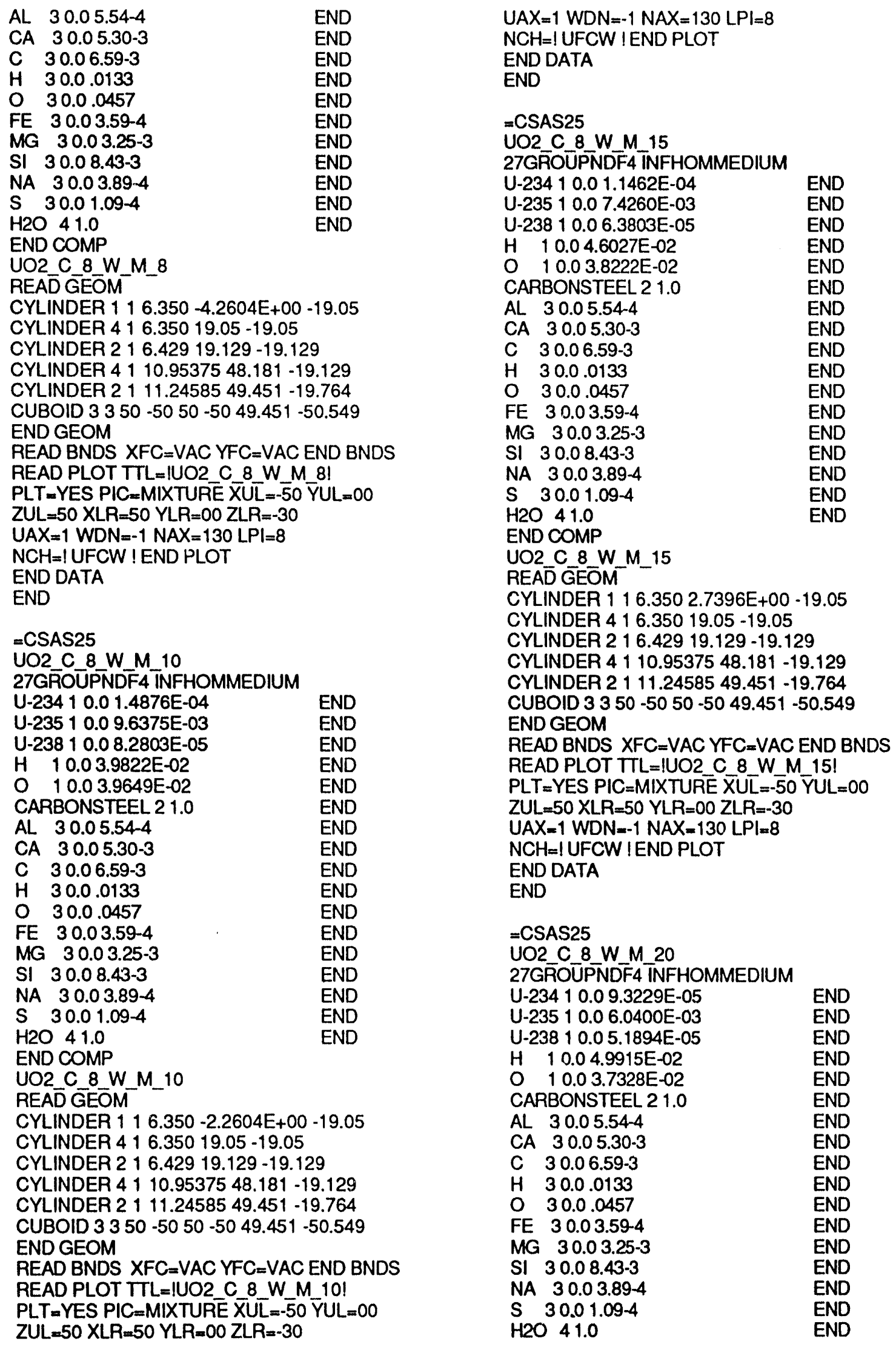




\begin{tabular}{|c|c|}
\hline \multicolumn{2}{|c|}{ 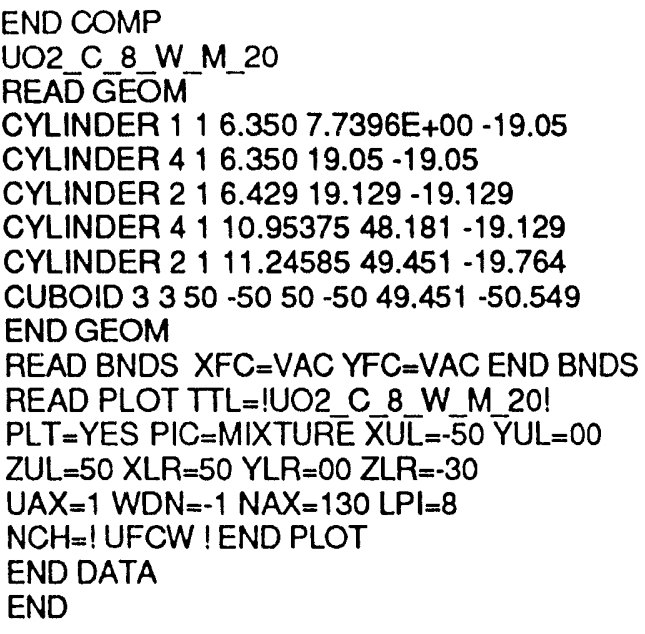 } \\
\hline 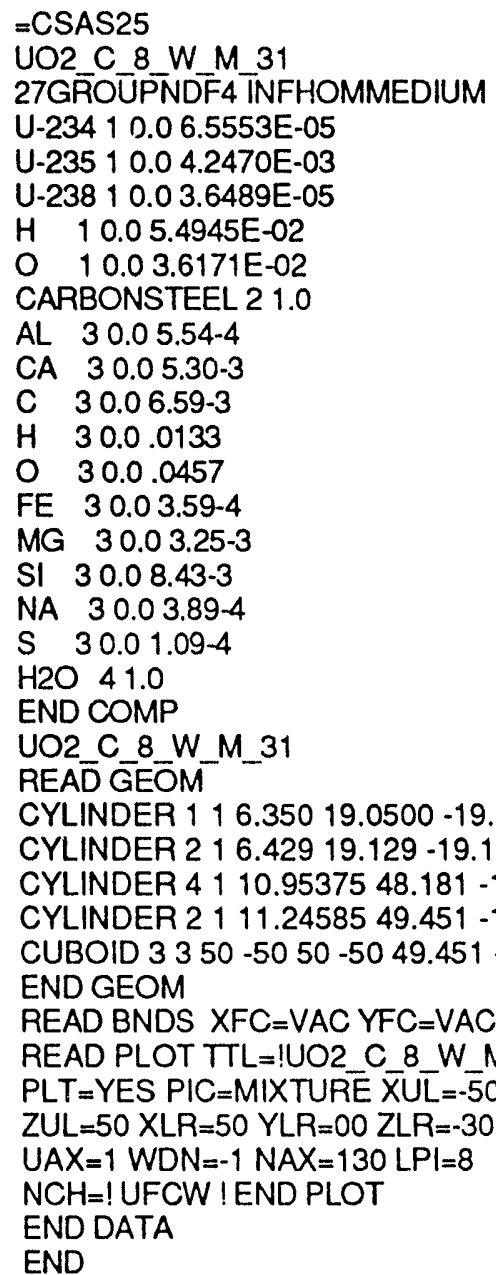 & $\begin{array}{l}05 \\
29 \\
19.129 \\
19.764 \\
-50.549 \\
\text { END BNDS } \\
131 ! \\
\text { YUL }=00\end{array}$ \\
\hline
\end{tabular}




\section{Appendix $\mathbf{G}$}

\section{X-345 Moderation Control Limits}

The moderation control limits for oxides in X-345 are given in a memo from the Portsmouth criticality safety staff in $1988^{1}$. These rules are summarized below:

1. Until notified otherwise in writing by the NCS staff, Nuclear Material Control personnel will neither assign below-floor-level storage positions in the oxide vault of X-345 for containers of uranium oxides, nor cause such containers to be placed in such positions, unless purities meet the requirements contained in correspondence 504-87-04 (table reproduced below).

2. Until notified otherwise in writing by the NCS staff, Nuclear Materials Control will send an inventory sheet - listing the ${ }^{235} \mathrm{U}$ assay, ${ }^{235} \mathrm{U}$ mass, and uranium purity of the uranium oxide in each container stored in the X345 oxide vault - to the NCS staff on a quarterly basis.

\section{Minimum Uranium Purity Required for Moderation} Control for Uranium Oxides ${ }^{2}$

\begin{tabular}{|c|c|c|c|}
\hline Material & $\mathrm{H}^{235} \mathrm{U}=65$ & $\mathrm{H} /{ }^{235} \mathrm{U}=15$ & $\mathrm{H} /{ }^{235} \mathrm{U}=10$ \\
\hline & \multicolumn{3}{|c|}{$235 \mathrm{U}$ Mass Limit(kg) } \\
\hline & 2 & 5 & 8 \\
\hline & \multicolumn{3}{|c|}{ Minimum Uranium Purity Limit $\left(\mathrm{gu}^{\mathbf{2 3}} \mathrm{g}_{\text {Total }}\right)$} \\
\hline $\mathrm{UO}_{2}$ & 0.276 & 0.585 & 0.660 \\
\hline $\mathrm{UO}_{3}$ & 0.271 & 0.560 & 0.630 \\
\hline $\mathrm{U}_{3} \mathrm{O}_{8}$ & 0.275 & 0.567 & 0.635 \\
\hline
\end{tabular}

\section{References}

1. Oxenham, R.L., Netzer, W. D., and Miller, R. R., Interim Moderation Control Limits for Oxides in X-345, Martin Marietta Energy Systems, Portsmouth, POEF-DM-1554, March 10, 1988.

2. D. A. Shisler, Martin Marietta Energy Systems, Portsmouth, Nuclear Materials Control, internal correspondence 504-87-04, January 9, 1987. 

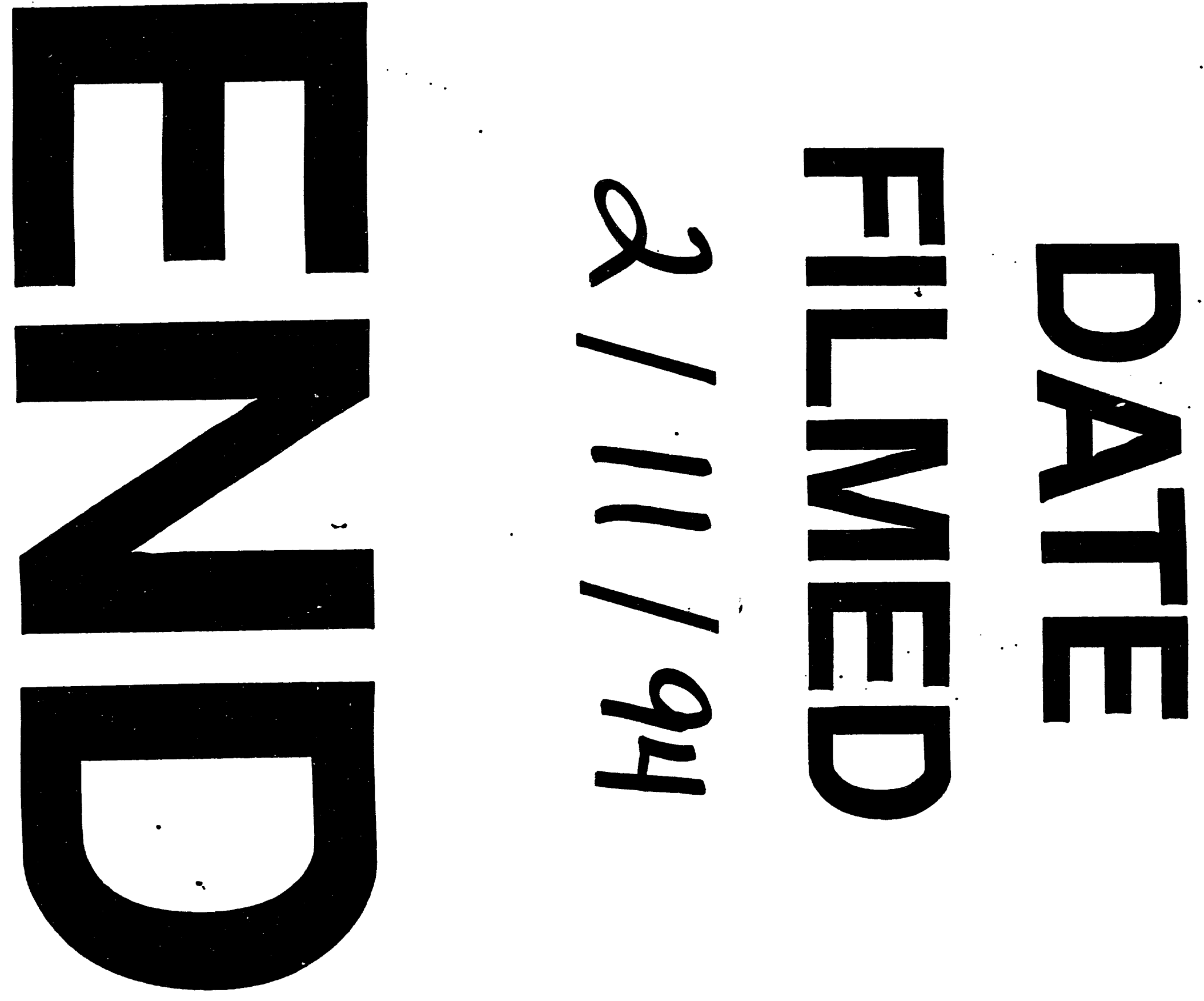
오 0

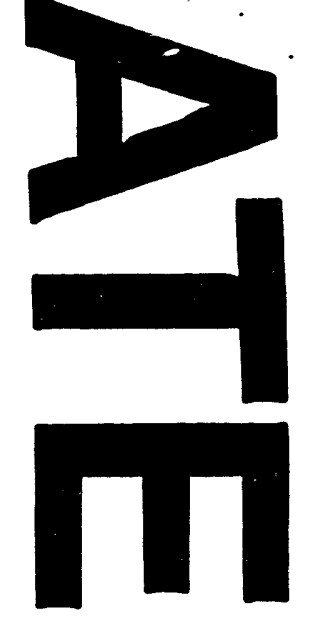




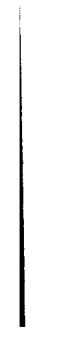

